


\section{Selected Topics in Plasma Physics}

Edited by Sukhmander Singh 

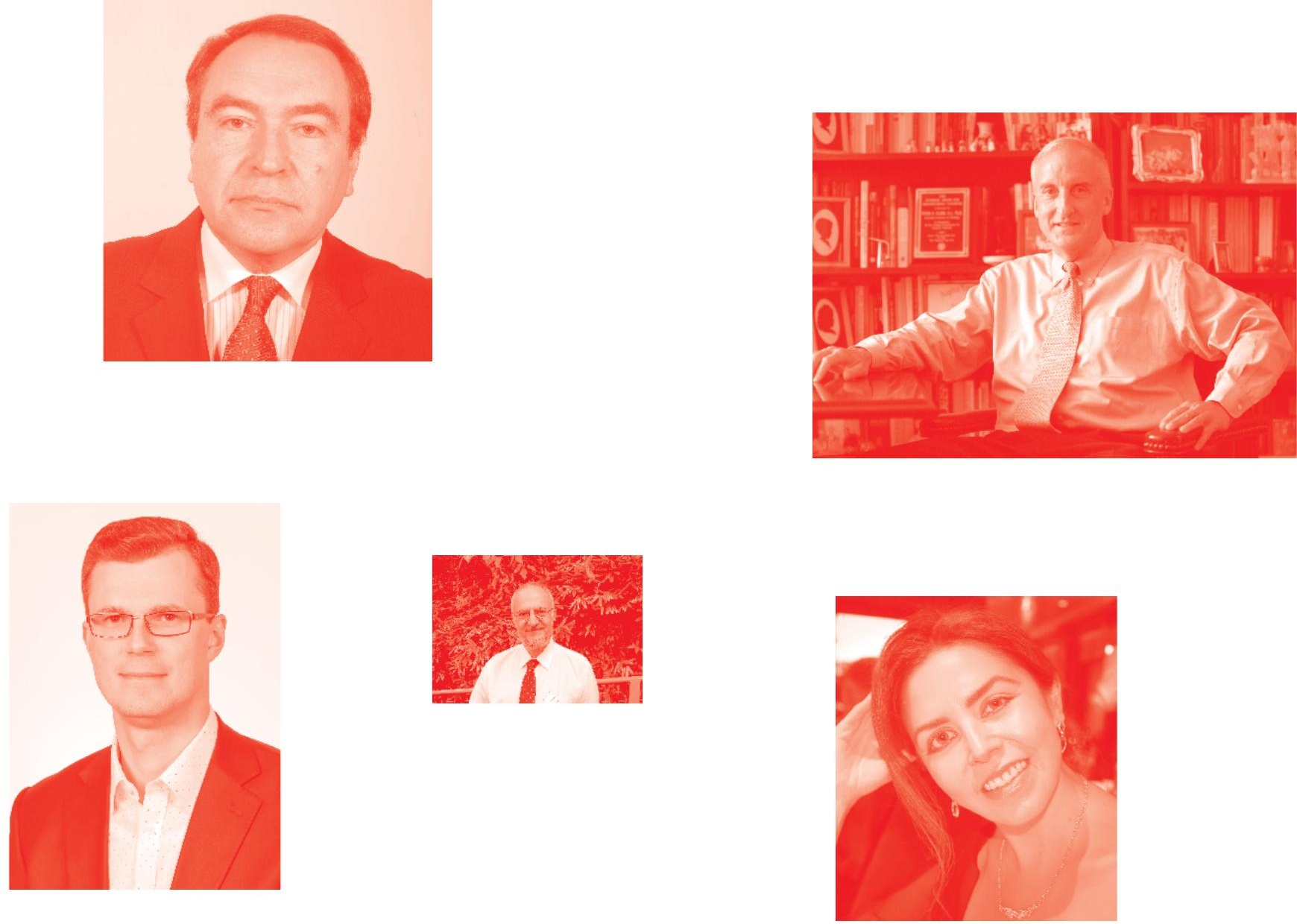

Supporting open minds since 2005
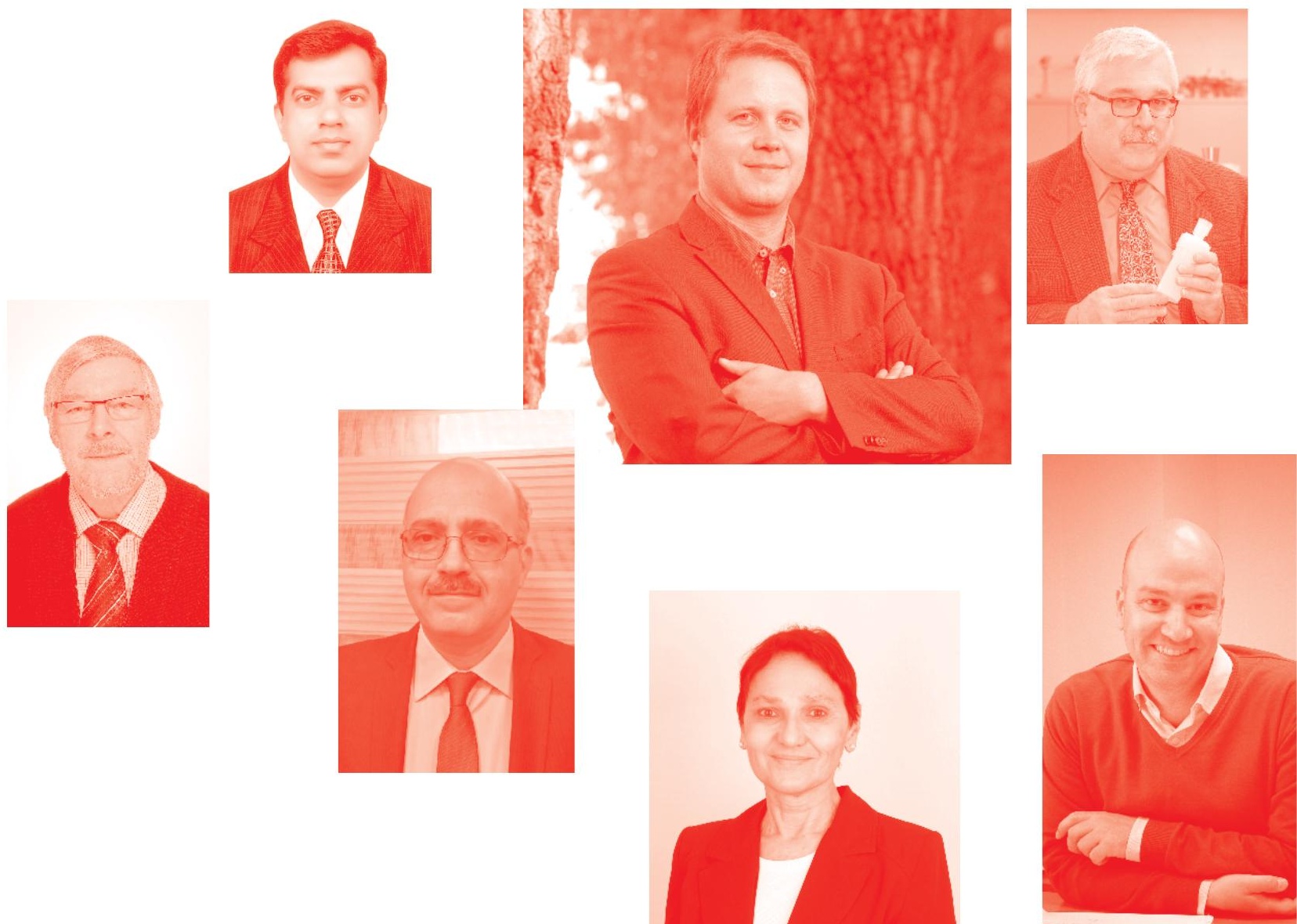
Selected Topics in Plasma Physics

http : //dx. doi. org/10.5772/intechopen. 78866

Edited by Sukhmander Singh

\section{Contributors}

Eduard Rostomyan, Theodore Anderson, Chinmoy Mallick, M. Bandyopadhyay, R Kumar, Sukhmander Singh, Shahid Ali, Yas Al-Hadeethi, Prasanta Chatterjee, Laxmikanta Mandi, Kaushik Roy

() The Editor(s) and the Author(s) 2020

The rights of the editor(s) and the author(s) have been asserted in accordance with the Copyright. Designs and Patents Act 1988. All rights to the book as a whole are reserved by INTECHOPEN LIMITED. The book as a whole (compilation) cannot be reproduced, distributed or used for commercial or non-commercial purposes without INTECHOPEN LIMITED's written permission. Enquiries concerning the use of the book should be directed to INTECHOPEN LIMITED rights and permissions department (permissions@intechopen.com).

Violations are liable to prosecution under the governing Copyright Law .

\section{(cc) BY}

Individual chapters of this publication are distributed under the terms of the Creative Commons Attribution 3.๑ Unported License which permits commercial use, distribution and reproduction of the individual chapters, provided the original author(s) and source publication are appropriately acknowledged. If so indicated, certain images may not be included under the Creative Commons license. In such cases users will need to obtain permission from the license holder to reproduce the material. More details and guidelines concerning content reuse and adaptation can be found at http : //www . intechopen. com/copyright-policy. html.

Notice

Statements and opinions expressed in the chapters are these of the individual contributors and not necessarily those of the editors or publisher. No responsibility is accepted for the accuracy of information contained in the published chapters. The publisher assumes no responsibility for any damage or injury to persons or property arising out of the use of any materials, instructions, methods or ideas contained in the book.

First published in London, United Kingdom, 2020 by IntechOpen

IntechOpen is the global imprint of INTECHOPEN LIMITED, registered in England and Wales, registration number: 11086078 , 5 Princes Gate Court, London, SW7 2QJ, United Kingdom Printed in Croatia

British Library Cataloguing-in-Publication Data

A catalogue record for this book is available from the British Library

Additional hard and PDF copies can be obtained from orders@intechopen.com

Selected Topics in Plasma Physics

Edited by Sukhmander Singh

p. cm.

Print ISBN 978-1-83962-678-4

Online ISBN 978-1-83962-679-1

eBook (PDF) ISBN 978-1-83962-680-7 


\section{We are IntechOpen, \\ the world's leading publisher of Open Access books}

\section{Built by scientists, for scientists}

\section{$5,100+$}

Open access books available

156

Countries delivered to
$126,000+$

International authors and editors

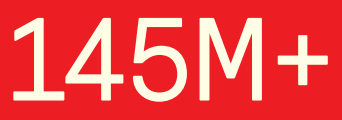

Downloads

Our authors are among the

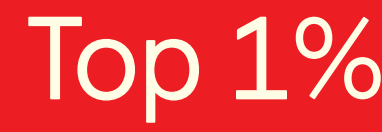

most cited scientists

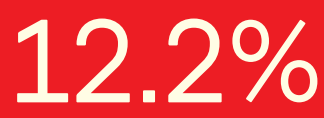

Contributors from top 500 universities

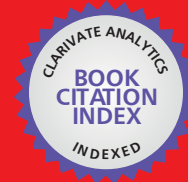

WEB OF SCIENCE ${ }^{\mathrm{TM}}$

Selection of our books indexed in the Book Citation Index in Web of Science ${ }^{\mathrm{TM}}$ Core Collection (BKCI)

Interested in publishing with us?

Contact book.department@intechopen.com

Numbers displayed above are based on latest data collected.

For more information visit www.intechopen.com

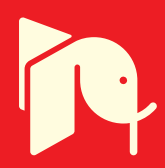





\section{Meet the editor}

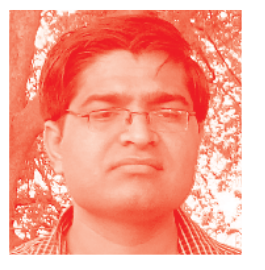

Dr. Sukhmander Singh is currently an assistant professor in the Department of Physics at Central University of Rajasthan, Ajmer, India. Previously, he was assistant professor at Motilal Nehru College, University of Delhi. He obtained his MSc (Physics) from Jawaharlal Nehru University New Delhi and PhD

(Plasma Physics) from IIT Delhi, New Delhi, India. He has published numerous research papers and conference proceedings in journals of international repute as well as contributed many book chapters. He is an active reviewer for many international journals. His areas of interest include theory and simulation of plasma waves and instabilities. 



\section{Contents}

$\begin{array}{ll}\text { Preface } & \text { XIII }\end{array}$

Section 1

Introduction to Plasma State

Chapter 1

Introduction: Plasma Parameters and Simplest Models

by Eduard Vardges Rostomyan

Section 2

Plasma Applications in Space Technology

Chapter 2

Hall Thruster: An Electric Propulsion through Plasmas

by Sukhmander Singh

Section 3

Microwave Plasma Interactions

Chapter 3

Evolution of Microwave Electric Field on Power Coupling to

Plasma during Ignition Phase

by Chinmoy Mallick, Mainak Bandyopadhyay and Rajesh Kumar

Section 4

69

Physics of Plasma Antennas

Chapter 4

Plasma Antennas

by Theodore Anderson

Section 5

Plasma Diagnostic Methods

Chapter 5

Plasma Diagnostic Methods: Test Charge Response in Lorentzian

Dusty Plasmas

by Shahid Ali and Yas Al-Hadeethi 
Section 6

Nonlinear Phenomena in Plasma

Chapter 6

Approximate Analytical Solution of Nonlinear Evolution Equations by Laxmikanta Mandi, Kaushik Roy and Prasanta Chatterjee 


\section{Preface}

Almost 99 percent of matter in the universe exists in a plasma state, as in the form of the Sun, stars, lightning, welding arcs, interplanetary and interstellar space, and in neon and fluorescent tubes. Plasma is an electrically conducting medium in which there are roughly equal numbers of neutral and positively and negatively charged particles.

This book introduces topics of plasma physics and presents related applied research. Several chapters deal with basic concepts in plasma physics, nonequilibrium plasma modeling, space plasma applications, and plasma diagnostics. The book also gives an overview of the linear and nonlinear aspects of plasma physics. As such, it is a useful resource for many young researchers and students in the field.

The first chapter provides an introduction to plasma physics, occurrence of plasma in universe, types of plasma, plasma quasi-neutrality, plasma frequency, Debye shielding, and waves in plasma. It also discusses stable and unstable plasma oscillations with the help of two fluids, also called plasma hydrodynamics.

Chapter 2 discusses the technological application of plasma physics in the field of electric propulsion. These devices have much greater exhaust velocities, longer lifetime, and greater thrust density than chemical propulsion devices. The chapter discusses different types of plasma propulsion devices by providing mathematical formulations. It also presents the current status of the research on Hall thrusters.

Chapter 3 discusses the interaction of microwave electric fields with plasma. It also gives the dispersion relations of electrostatic and electromagnetic waves. The spatio-temporal evolution pattern of microwave-radiated plasma parameters highlight the role of these electric fields in power coupling processes.

Chapter 4 deals with the design of smart plasma antennas to achieve high gain and reconfigurable beam width without physically moving the antenna. It also reports on the generation of microwaves and their reflection from plasma.

Chapter 5 explains the principles of the diagnostic technique for a test charge in Lorentzian dusty plasma with the help of space-time Fourier transformations. Finally, Chapter 6 deals with the theory of nonlinear waves in plasma to obtain the Korteweg-de Vries (KDV) equation by using the Reductive Perturbation Technique.

We are grateful to our authors Rostomyan, Mallick, Bandyopadhyay, R. Kumar, Theodore Anderson, S. Ali, Y. Al-Hadeethi, Laxmikanta Mandi, Kaushik Roy, and Prasanta Chatterjee. I especially thank Mr. Anil Joshi, who always gave me good advice and helped on several occasions. Special thanks to my parents, sisters, grandfather, and wife Rajvinder Kaur for her cooperation in my life and for sharing 
the responsibility of family affairs so that I could spend my time for this book. I gratefully acknowledge the technical support provided by the publisher IntechOpen for bringing out this book in a very short time.

Dr. Sukhmander Singh

Assistant Professor,

Department of Physics,

Central University of Rajasthan,

Ajmer, Kishangarh, India 
Section 1

Introduction to Plasma State 



\title{
Introduction: Plasma Parameters and Simplest Models
}

\author{
Eduard Vardges Rostomyan
}

\begin{abstract}
Plasma is ionized gas (partially or fully). Overwhelming majority of matter in the universe is in plasma state (stars, Sun, etc.). Basic parameters of plasma state are given briefly as well as classification of plasma types: classic-quantum, idealnonideal, etc. Differences between plasma and neutral gas are presented. Plasma properties are determined by long distance electrostatic forces. If spatial dimensions of a system of charged particles exceed the so-called Debye radius, the system may be considered as plasma, that is, a medium with qualitatively new properties. The expressions for Debye radius for classical and quantum plasma are carried out. Basic principles of plasma description are presented. It is shown that plasma is a subject to specific electrostatic (or Langmuir) oscillations and instabilities. Simplest plasma models are given briefly: the model of "test" particle and model of two (electron and ion) fluids. As an example, Buneman instability is presented along with qualitative analysis of its complicate dispersion relation. Such analysis is typical in plasma theory. It allows to easily obtain the growth rate.
\end{abstract}

Keywords: plasma, quasi-neutrality, Langmuir frequency, Debye length, simplest plasma models, unstable plasma oscillations

\section{Introduction}

Everyone knows the three states of matter: solids, liquids, and gases. Plasma is often called the fourth state of matter; bear in mind that with increasing temperature, the following transitions take place: solids-liquids-gases-plasma. Under the last transition, atoms lose electrons. Plasma consists (along with neutral atoms) of charged particles: electrons and positively charged ions (single and/or multiple ionized). This definition of plasma is far from complete. The complete definition of plasma is, in fact, impossible. It must cover a very wide range of phenomena in a wide variety of conditions.

Plasma is very common in the universe. Most of the substance in it (more than 99\%) is in plasma state. Media consisting of ionized atoms is found almost everywhere. The upper layers of the Earth's and stellar atmospheres, interstellar medium, etc. actually are in plasma state. Stellar plasma is another widespread example. In the plasma of stars, in particular the Sun, reactions of the synthesis of light elements, the so-called thermonuclear reactions, provide a huge release of energy and plasma heating. Currently, scientists from many countries around the world are studying the possibility of creating such a high-temperature plasma in terrestrial conditions, setting the task of implementing controlled thermonuclear fusion and providing humanity with an inexhaustible supply of energy. 
There are two fundamentally different approaches to the implementation of controlled thermonuclear fusion. The first approach is obtaining the reactions in the so-called magnetic traps. Hot plasma should not come in contact with the walls of the chamber, as this will lead to its actual destruction. The confinement of the plasma by a magnetic field, in theory, should prevent the contact of the plasma with the walls of the chamber, since the magnetic field bends the trajectory of charged particles. However, despite tremendous efforts, the task of plasma confining has not been completely solved yet. Special configurations of magnetic field, magnetic traps help only partially. Plasma is an unstable medium in which small perturbations increase and destroy its given state. Instabilities are intrinsic for plasma. It turned out that any nonequilibrium initial distribution of particles is unstable. Below we show how instability follows from general electrodynamical consideration and give an example.

The second approach is the very quick heating of plasma up to thermonuclear temperatures. The reaction itself and energy removal also take place quickly. These processes recur very fast, and confinement of such plasma is not needed. This approach is called inertial fusion. It was proposed when very fast heating of plasma becomes possible by using intense laser beams and/or high-current relativistic electron beams.

The development of these studies is associated with the rebirth of the concept of plasma, which arises upon the investigations of gas-discharge processes. The processes in gas-discharge plasma have also been intensively studied. The studies were associated with the development of the needs of classical and quantum electronics, for which gasdischarge appliances play an important role. Finally, solid-state plasma should be noted: electron plasma of metals and electron-hole plasma of semiconductors.

The listed series can be continued almost unlimitedly, speaking about plasma in magnetohydrodynamic and thermionic converters of thermal energy into electrical energy, about plasma in solutions of electrolytes, etc. However, the above examples are sufficient to make sure the extremely wide prevalence of plasma in nature and the importance of studying its properties.

A vast literature has been grown to describe plasma state (see, e.g., [1-7] and many others). Our further presentation is based on the principles of plasma electrodynamics considering plasma as a continuous medium with a large number of free charged carriers.

\section{Plasma parameters}

Plasma is an ionized gas consisting of free electrons and various types of ions and neutrals. First of all, it is necessary to know the charge $e_{\alpha}$ and concentration $n_{\alpha}$ of plasma components (here the index takes different values corresponding to the types of particles in the plasma, $\alpha=e$ for electrons, $\alpha=i_{1}, i_{2} \ldots$ for ions of various types, and $\alpha=n$ for neutrals). All plasma particles are in chaotic motion, but full thermodynamic equilibrium is absent. Usually each component has its own temperature $T_{\alpha}$, which is also necessary to know.

In solid-state and semiconductor plasma, the conception of temperature should be given more accurately. If the Fermi energy $E_{F \alpha}$ of $\alpha$-type particles exceeds their thermal energy

$$
E_{F \alpha}=\frac{\left(3 \pi^{2}\right)^{2 / 3} \hbar^{2} n_{\alpha}^{2 / 3}}{2 m_{\alpha}} \gg T_{\alpha}
$$

(here $m_{\alpha}$ is the mass of the particle, and $\hbar$ is the Planck constant), quantum effects should be accounted. In this case the Maxwell's distribution function does not describe the behavior of charged particles. It should be described by Fermi distribution function, and the $E_{F \alpha}$ (1) plays the role of temperature. In this case plasma is degenerate. 
If neutrals are absent, plasma is fully ionized. In the opposite case, plasma is partially ionized, and it is necessary to know the level of plasma ionization. This is the ratio of neutrals' density to the density of charged particles (or to the full density of plasma).

An important characteristic peculiarity of plasma state is a very wide range of values of these (and other) parameters. For example, plasma in some stars (white dwarfs) has a density of $10^{25}-10^{26} \mathrm{~cm}^{-3}$, but in the interstellar space, plasma has a density of $1-10 \mathrm{~cm}^{-3}$. The ratio is $10^{26}$. The ratio of other parameter values is a bit less. This leads to important consequences. In the example above, different approaches may be required to describe the plasma inside the stars and in the interstellar space. The most interesting cases will be mentioned below.

An important condition for plasma existence is its quasi-neutrality. The condition of quasi-neutrality has the form

$$
\sum_{\alpha} e_{\alpha} n_{\alpha} \approx 0
$$

where the summation is made over all types of charged particles $\alpha=e, i_{1}, i_{2}, i_{3} \ldots$ When it is violated, strong electric fields arise, which restore plasma quasineutrality. Violations of quasi-neutrality are possible only in spatial and temporal scales, small in comparison with the characteristic plasma scales. The temporal characteristic scale of plasma is determined by its proper oscillations, but the spatial scale is determined by the length of plasma shielding (Debye length; see below).

\subsection{Plasma oscillations}

\subsubsection{Langmuir frequency}

Plasma, as a medium with a large number of free charged particles, is a subject to oscillations. Consider in detail the oscillations of uniform electron plasma. Ions are heavy (immobile) and serve for charge neutralization. Let a small displacement of an electron layer relative to the ions take place (see Figure 1). We denote the displacement vector by $\mathbf{X}$. The density of the uncompensated electron charge at the displacement $\mathbf{X}$ may be found from the continuity equation:

$$
\rho=\nabla n_{e} e \mathbf{X}=n_{e} e \nabla \mathbf{X}
$$

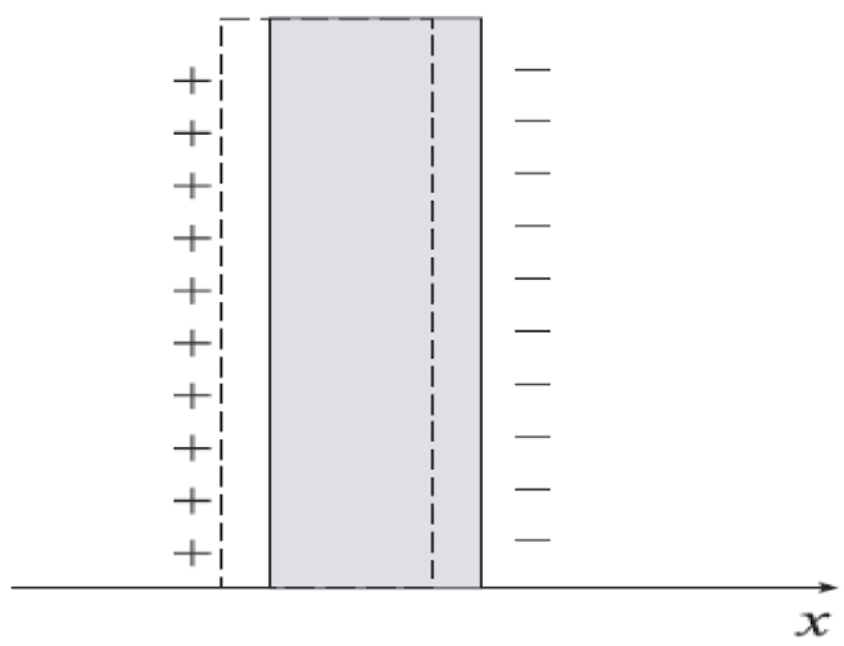

Figure 1.

Oscillations of the electron layer. 
This charge creates an electric field $\mathbf{E}$, the value of which may be determined from Poisson's equation:

$$
\nabla \cdot \mathbf{E}=4 \pi \rho=4 \pi n_{e}|e| \nabla \cdot \mathbf{X}
$$

Hence, we can write (given that for $\mathbf{X}=0$, we have $\mathbf{E}=0$ )

$$
\mathbf{E}=4 \pi n_{e} e \mathbf{X}
$$

Thus, the field $\mathbf{E}$ is parallel to the displacement of electrons and acts on each electron with a force

$$
\mathbf{F}=-e \mathbf{E}=-4 \pi n_{e} e^{2} \mathbf{X}
$$

tending to return the electron to its original equilibrium position. As a result, we have the equation of motion of an electron in the form

$$
m \frac{d^{2} \mathbf{X}}{d t^{2}}=-e \mathbf{E}=-4 \pi n_{e} e^{2} \mathbf{X}
$$

This equation describes the oscillations of plasma electrons near the equilibrium position $(\mathbf{X}=0)$ with a frequency

$$
\omega=\omega_{L e}=\sqrt{\frac{4 \pi n_{e} e^{2}}{m}}
$$

which is known as the electron Langmuir frequency. If one uses MKS units, the expression for electron Langmuir frequency is

$$
\omega_{L e}=\sqrt{\frac{n_{e} e^{2}}{\varepsilon_{0} m}}
$$

where $\varepsilon_{0}$ is the dielectric permittivity of vacuum. Violations of plasma quasineutrality are possible only on a temporal scale, small in comparison with time $\tau \sim 1 / \omega_{L e}$.

\subsection{Gas parameter. Debye length}

The behavior of an ionized gas is determined by long-distance electrostatic forces. These forces significantly influence on the plasma behavior and, actually, determine its parameters. First of all it is necessary to find out under what conditions a system of electrostatically interacting particles can be considered as a gas. The main peculiarity of a gas is the following: its particles interact during very small time intervals only (during collisions); the rest time every particle moves independently on others. At distances exceeding the size of the gas molecules, there is no interaction (its potential is equal to zero). Or, in other words, the potential energy of a particle is much lesser than their kinetic energy. In this case, the ratio of the distance, at which the interaction between the particles is significant to the average distance between particles, is small [8]:

$$
\Lambda_{G}=\frac{a}{\langle r\rangle} \approx a n^{1 / 3}<<1
$$

(here $a$ is the molecule size, $\langle r\rangle$ is the average distance between particles, and $n$ is the density). The condition (10) also holds for the interaction of electrons with 
neutrals and ions with neutrals. However, if we consider long-distance interaction between charge particles, the parameter $\Lambda_{G}$ (gas-like parameter) requires rethinking. Its physical meaning becomes slightly different. The gas approximation is valid if the energy of the interaction between particles $U(\langle r\rangle)$ is smaller than the average thermal energy $T$ of the particles itself, i.e.

$$
U(\langle r\rangle) \approx \frac{e^{2}}{\langle r\rangle}<<T
$$

In other words, the following parameter, determining the plasma state

$$
\Lambda_{G} \rightarrow \Lambda_{P}=\frac{U(\langle r\rangle)}{T} \approx \frac{e^{2} /\langle r\rangle}{T} \approx \frac{e^{2} n^{1 / 3}}{T} \ll 1
$$

must be small. The first condition (for a neutral gas) means that in a sphere with a radius equal to the radius of interaction, there are few particles. The meaning of a similar condition for plasma is the opposite. To prove this we, first of all, determine the interaction radius in plasma. For the determination we consider in detail the potential of a test particle in plasma. Let a particle with a charge $q$ be placed at the point $r=0$. We intend to find its potential $\varphi$ from Poisson's equation. Assuming, for simplicity, that the charge of the single type of singly charged ions is not changed by the test particle (i.e., $e_{i}=-e$ ), we can write Poisson's equation in the form

$$
\Delta \varphi=-4 \pi q \delta(r)-4 \pi e\left[n_{e} \exp \frac{-e \varphi}{T_{e}}-n_{i} \exp \frac{e \varphi}{T_{i}}\right]
$$

where $\Delta$ is the Laplace operator and $\delta(r)$ is the Dirac function. Assuming that $|e \varphi|<<T_{e}, T_{i}$, we find

$$
\varphi(r)=\frac{q}{r} \exp \left(-\frac{r}{r_{D}}\right)
$$

where

$$
r_{D}=\left\{\sum_{\alpha}\left(\mathrm{v}_{\mathrm{T} \alpha}^{2} / \omega_{L \alpha}^{2}\right)^{-1}\right\}^{-\frac{1}{2}}
$$

is the so-called Debye radius. It shows the distance, in which the Coulomb forces are acting in plasma. Outside of the Debye radius, the interaction between charged particles is exponentially small and may be neglected. Comparative characteristics of the two curves are given in Figure 2. Curve (a) presents Debye potential, and curve (b) presents the vacuum potential $\sim 1 / r$.

The electrostatic forces are, in fact, shielded. Now we can compare the average distance between charged particles with the Debye radius and make sure that the number of particles in the Debye sphere is large. For a simple case of plasma with singly charged ions and $T_{e} \approx T_{i} \approx T$, we have

$$
r_{D} n^{1 / 3}=n \sqrt{\frac{T_{e} T_{i}}{4 \pi\left(e^{2} n_{e} T_{i}+e_{i}^{2} n_{i} T_{e}\right)}} \approx \frac{\sqrt{T}}{e n^{1 / 6}}=\sqrt{\frac{1}{\Lambda_{P}}}>1
$$

This condition is essentially the opposite of analogous condition for gas (10). In a gas, the particles generally do not interact. The interaction takes place only at very 


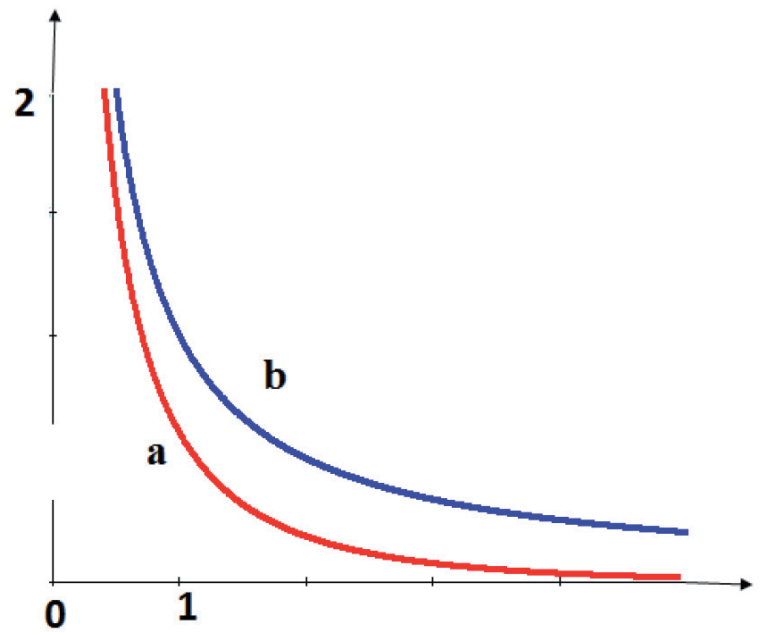

Figure 2.

Comparative characteristic of the two curves. Curve (a) presents Debye potential, and curve $(b)$ presents the vacuum potential.

short intervals during collisions. In plasma, on the contrary, particles experience an interaction almost always. But, at the same time, the interaction is weak. It does not outrage their movement.

Debye radius, in particular, for electrons is

$$
r_{D e}=\frac{\mathrm{v}_{T e}}{\omega_{L e}}=\sqrt{\frac{T_{e}}{4 \pi n_{e} e^{2}}}
$$

For the quasi-neutrality of plasma, it is necessary that its characteristic dimensions $L$ be much larger than the Debye radius $L \gg r_{D}$. Moreover, under this condition a system of charged particles can be considered as plasma, i.e., a material medium with qualitatively new properties. Otherwise, it is a simple collection of individual charged particles, to which vacuum electrodynamics is applicable.

\subsection{Degenerate plasma}

It remains to determine the gas-like parameter for degenerate plasma as well as to answer the question of whether is there in quantum plasma Debye screening. For this we first recall that the expression for average energy of the particles, which is valid both for classical and quantum cases, may be written in the following form:

$$
\langle E\rangle=\left\{\begin{array}{lll}
T & \text { if } & T>E_{F} \\
E_{F} & \text { if } & E_{F}>T
\end{array}\right.
$$

i.e., in the quantum case, the average energy of the state is equal to Fermi energy $E_{F}$ (see (1)). The gas-like parameter for degenerate plasma may be obtained if we replace $T \rightarrow E_{F}$ in the expression (12). It becomes

$$
\Lambda_{P}^{(D)}=\frac{e^{2} n^{1 / 3}}{E_{F}} \ll 1
$$

Now we show that in quantum (degenerate) plasma of metals, shielding of electrostatic field also takes place and derives an expression for characteristic length for the Debye radius in degenerate plasma. 
The energy of free electrons is $p^{2} / 2 m$. In the presence of a field with the potential $\Phi(r)$, it is $p^{2} / 2 m+\Phi(r)$. As a result, Fermi particles become distributed uniformly in the spherical layer between $p_{\min }=\sqrt{2 m e \Phi}$ and $p_{\max }=\sqrt{p^{2}+2 m e \Phi}$. Given this circumstance, one can find the expression for the electron density:

$$
n_{e}=n_{0 e}\left(1+\frac{e \Phi}{E_{F e}}\right)^{3 / 2},
$$

where $n_{0 e}$ is the density in the absence of a field (which coincides with the density of the neutralizing ion background). Now it is not difficult to write Poisson's equation for the potential of the test particle placed at the point $r=0$, and the charge of which is $q$. The equation is

$$
\Delta \Phi=-4 \pi q \delta(\mathbf{r})-4 \pi e n_{0 e}\left[\left(1+\frac{e \Phi}{E_{F e}}\right)^{3 / 2}-1\right]
$$

The solution of this equation in the limit of weak fields, $|e \Phi|<<E_{F e}$, gives the shielded Coulomb potential:

$$
\Phi(r)=\frac{q}{r} \exp \left(-\frac{r}{r_{D e}}\right)
$$

with a Debye radius $r_{D e}$, the expression for which is

$$
r_{D e}=\sqrt{\frac{E_{F e}}{6 \pi e^{2} n_{0 e}}}
$$

The gas parameter for plasma (12) is similar to condition (10) for neutral gas in the following sense. Both of these conditions are fulfilled better for fewer densities of plasma and neutral gas. The better the gas condition is satisfied, the more ideal is plasma. For degenerate plasma (in which particles need quantum description), in the contrary, the gas condition depends on density inversely, i.e., with the increase in density, the ideality becomes better (see (19). As $E_{F} \sim n^{2 / 3}$, it turns out that with the increase in density, the average energy of Coulomb interaction increases slower. As a result $\Lambda_{G}^{(D)} \sim n^{-1 / 3}$. So, the denser the degenerate metal component, the better gas condition is fulfilled for it.

The diagram below presents the areas of the charge carriers' degeneracy and areas of the gas approximation applicability.

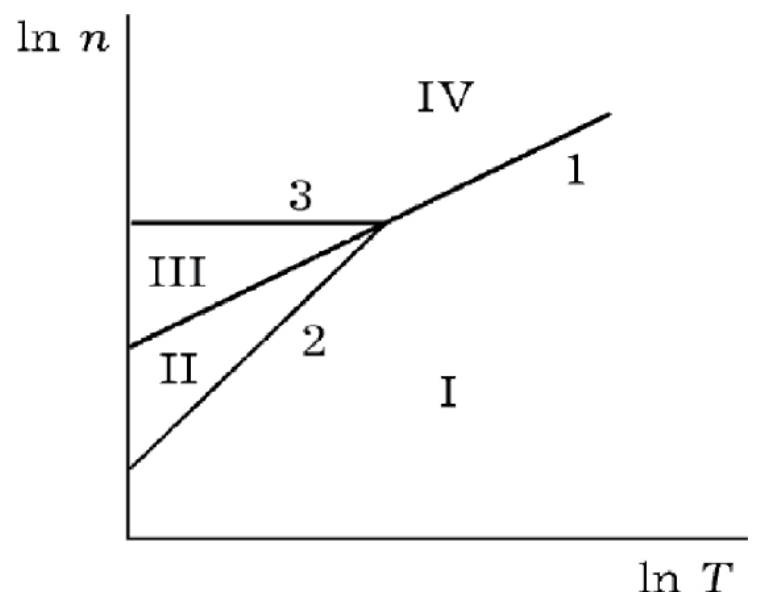


The degeneracy condition for electron plasma has the form $E_{F}>T\left(E_{F}\right.$; see (1)). In the diagram $\ln n$ vs $\ln \mathrm{T}$, this condition gives line 1 dividing the region of the degenerate plasma from the nondegenerate (classical) state. The condition for the applicability of the gas approximation in a nondegenerate state is $\Lambda_{P}=\frac{e^{2} n^{1 / 3}}{T}<<1$. In the same diagram, the condition $\Lambda_{P}=1$ gives line 2 . In the degenerate state, the condition for applicability of gas approximation is $\Lambda_{P}^{(D)}=e^{2} n^{1 / 3} / \mathrm{E}_{F}<<1$, in which $E_{F}$ does not depend on $T$. In these conditions $\Lambda_{P}^{(D)}=1$ gives line 3 passing through the point, where lines $1\left(E_{F}=T\right)$ and $2\left(\Lambda_{P}=1\right)$ intersect. Therefore, region I is the region of a nondegenerate plasma with weak interaction (the gas approximation is applicable). Region II is the region in which the plasma is nondegenerate with strong interaction, i.e., classic fluid. In region III, the plasma is degenerate with strong interaction, i.e., quantum fluid. Both in region II and region III, the gas approximation is not applicable. Finally, region IV of the parameter variations characterizes degenerate plasma with a weak interaction (gas approximation is applicable).

In conclusion, we give an estimate of the applicability conditions for the gas approximation (10) and (12) for various plasmas. First of all, we note that the size of atoms and molecules is of order $a \sim 10^{-7}-10^{-8} \mathrm{~cm}$ and the condition for gas approximation (10) is satisfied up to $n<10^{21}-10^{22} \mathrm{~cm}^{-3}$, i.e., in gases at normal temperature up to a pressure of hundred atmospheres. It is obvious that in gas plasma both in the ionosphere and in the laboratory, this condition is fulfilled perfectly, with a large margin.

A somewhat different situation holds for the condition of gas approximation in plasma (12). In the ionosphere plasma, where $n_{e} \sim 10^{6}-10^{7} \mathrm{~cm}^{-3}$ and $T_{e} \approx 10^{4} \mathrm{~K}$, we have $\Lambda_{P} \approx 10^{-4} \ll 1$, i.e., the condition is well satisfied. In ordinary gas-discharge fluorescent lamps, as well as in discharges used in laboratory experiments, where $n_{e} \approx 10^{10}-10^{14} \mathrm{~cm}^{-3}$ and $T_{e} \sim 10^{4}-10^{5} \mathrm{~K}$, the value of $\Lambda_{P}<<1$. However, at the discharge in dense gases used in the light sources for laser pumping, as a rule, $n_{e} \ll 10^{18}-10^{19} \mathrm{~cm}^{-3}$, and $T_{e} \ll 1-10 \mathrm{eV}$. Herewith $\Lambda_{P} \approx 0.1-0.5$, which indicates a significant violation of the applicability condition for the gas approximation and a significant manifestation of the properties of non-ideal plasma or, as one says, liquid effects.

In a thermonuclear plasma, in facilities with magnetic confinement, $n_{e} \approx 10^{14}-10^{15} \mathrm{~cm}^{-3}$, and $T_{e} \approx T_{i} \approx 10^{8} \mathrm{~K}$. As a result, we have $\Lambda_{P} \approx 10^{-5}<<1$, i.e., the ideality of plasma is guaranteed. However, in the inertial thermonuclear reactors, where experimentators strive to obtain plasma with $n_{e} \sim n_{i} \sim 10^{24}-10^{25} \mathrm{~cm}^{-3}$ at the temperature of $\mathrm{T} \approx 10^{8} \mathrm{~K}$, it turns out that $\Lambda_{P} \geq 0.01$ and may be even more. This, apparently, will require a consideration of weakly non-ideal plasma, especially in conditions of pollution (the presence of multiply charged ions).

Finally, a brief summary on ideality of plasma in solids is presented. Even in good conductors, such as copper, where $n_{e} \approx 5 \cdot 10^{22} \mathrm{~cm}^{-3}$ and $E_{F} \sim 1 \mathrm{eV}$, we have $\Lambda_{P} \equiv$ $\Lambda_{p}^{(D)} \sim 1$, i.e., plasma of metals is always non-ideal, and it is more correct to consider it as electron liquid. Nevertheless, it turns out that the application of the gas approximation to metals leads to good results from the point of view of the comparison with experiments. As for the electron-hole plasma of semiconductors, they are not degenerate at normal temperature, due to the small density of the carriers. For this reason, the condition of gas approximation (19) is well satisfied. Exceptions can occur only at very low temperatures.

\section{Plasma description}

\subsection{Self-consistent approach}

The main feature of plasma and plasma-like media, such as gas plasma, plasma of metals, semimetals, and semiconductors, is the presence of a large number of free 
charge carriers. Here we present general principles of their description as a continuous media. The term "plasma-like media" was first introduced in [6] (see also [7]), the authors of which understood such seemingly different states of matter as an ionized gas, or actually plasma; metals, semiconductors, and even molecular colloidal crystals and electrolytes may be described based on similar principles - the principles of electrodynamics of plasma-like media. In this section we briefly formulate these principles.

A self-consistent interaction of the electromagnetic field and charge carriers takes place in plasma-like media. Field equations are the equations of Maxwell in which the current and the charge must be represented by a sum over all carriers (charged particles) in the plasma:

$$
\begin{aligned}
& \nabla \times \mathbf{B}=\frac{1}{c} \frac{\partial \mathbf{E}}{\partial t}+\frac{4 \pi}{c} \mathbf{j}=\frac{1}{c} \frac{\partial \mathbf{E}}{\partial t}+\frac{4 \pi}{c} \sum_{\alpha} n_{\alpha} \mathbf{v}_{\alpha} e_{\alpha} \\
& \nabla \times \mathbf{E}=-\frac{1}{c} \frac{\partial}{\partial t} \mathbf{B} \\
& \nabla \mathbf{E}=4 \pi \rho=4 \pi \sum n_{\alpha} e_{\alpha} ; \quad \nabla \mathbf{B}=0
\end{aligned}
$$

where $\mathbf{E}$ is the electric field strength, $\mathbf{B}$ is the magnetic induction, and $n_{\alpha}, e_{\alpha}$, and $\mathbf{v}_{\alpha}$ are the density, charge, and the velocity of $\alpha$-th carrier $\alpha=e, i_{1}, i_{2}, i_{3} \ldots \quad$..

The equations for fields are written in this form (i.e., in terms of $\mathbf{E}$ and $\mathbf{B}$ ), because these quantities have direct physical meaning: they determine the Lorentz force $\mathbf{F}_{n}$ that acts on $n$-th carrier of $\alpha$ type (it may be an electron or ion of arbitrary type):

$$
\mathbf{F}_{n}=e_{\alpha}\left\{\mathbf{E}+\frac{1}{c}\left[\mathbf{v}_{n} \times \mathbf{B}\right]\right\}
$$

According to charge conservation law, the continuity equation must be satisfied for electrons and all types of ions, i.e., for $\alpha=e, i_{1}, i_{2}, \ldots$ The continuity equation is

$$
\frac{\partial n_{\alpha}}{\partial t}+\nabla\left(n_{\alpha} \mathbf{v}_{\alpha}\right)=0
$$

Here and in consideration below, we do not take into account the processes of ionization and recombination.

Eqs. (24)-(26) describe the self-consistent interaction between electromagnetic fields and statistically large numbers of charged particles (plasma). According to the set, electromagnetic fields determine the motion of charged particles. In its turn, the same electromagnetic fields are induced by moving plasma particles.

If the plasma (or plasma-like media) are in external fields (electric $\mathbf{E}_{0}$ and/or magnetic $\mathbf{B}_{0}$ ), the equations must be written in somewhat other form. External fields should be singled out. If the external fields are excited by external current $\mathbf{j}_{0}$ and charge $\rho_{0}$ densities, the set (24) should be written as

$$
\begin{aligned}
& \nabla \times \mathbf{B}=\frac{1}{c} \frac{\partial \mathbf{E}}{\partial t}+\frac{4 \pi}{c}\left(\mathbf{j}_{0}+\mathbf{j}\right)=\frac{1}{c} \frac{\partial \mathbf{E}}{\partial t}+\frac{4 \pi}{c}\left\{\mathbf{j}_{0}+\sum_{\alpha} n_{\alpha} \mathbf{v}_{\alpha} e_{\alpha}\right\} \\
& \nabla \mathbf{E}=4 \pi\left(\rho+\rho_{0}\right)=4 \pi \sum n_{\alpha} e_{\alpha}+4 \pi \rho_{0} \\
& \nabla \mathbf{B}=0 ; \quad \nabla \times \mathbf{E}=-\frac{1}{c} \frac{\partial}{\partial t} \mathbf{B}
\end{aligned}
$$


The external current $\mathbf{j}_{0}$ and charge density $\rho_{0}$ do not depend on the processes in plasma. Their values, along with $\mathbf{E}_{0}$ and $\mathbf{B}_{0}$, satisfy Maxwell's equations. These fields also influence on the motion of plasma particles. The expression for Lorentz force looks like (25)

$$
\mathbf{F}_{n}=e_{\alpha}\left\{\mathbf{E}+\frac{1}{c}\left[\mathbf{v}_{n} \times \mathbf{B}\right]\right\}
$$

However, here fields $\mathbf{E}$ and $\mathbf{B}$ are induced by external charge and current also.

An important conclusion follows from the sets (24) and (27). Only one additional vector quantity appears in the field equations - the current in the plasma $\mathbf{j}$ (the charge density may be expressed in terms of $\mathbf{j}$ by solving the continuity equation):

$$
\mathbf{j}=\mathbf{j}(\mathbf{E})=\sum_{\alpha} n_{\alpha} e_{\alpha} \mathbf{v}_{\alpha}
$$

In this expression $\mathbf{v}=\mathbf{v}(\mathbf{E})$. The expression (29) shows that the current, induced in plasma, depends on velocities $\mathbf{v}_{\alpha}$ which are found independently (e.g., from equations of motion). ${ }^{1}$ In the following, we will consider linear phenomena only. This implies linear dependence $\mathbf{j}(\mathbf{E})$, which is true, if the fields are comparatively small. It is to the point to note that in spite of our (and most other) consideration, nonlinear effects reveal themselves, first of all, in plasma and plasma-like media. Under linear consideration in isotropic media (media with no preferred directions), we actually have proportional dependence of the plasma current on the electric field or

$$
\mathbf{j}=\sigma \mathbf{E}
$$

This dependence represents Ohm's law for plasma, and $\sigma$ is plasma conductivity for the considered case of isotropic plasma. However, if the plasma is in the external field, it loses the isotropy, and the relationship between $\mathbf{j}$ and $\mathbf{E}$ becomes much more complicated.

\subsection{Waves in plasma and plasma-like media}

The most important solutions of formulated set of equations are the solutions in the form of traveling waves, i.e., the solutions that depend on space coordinate $\mathbf{r}$ and time $t$ as

$$
\sim \exp (-i \omega t+i \mathbf{k r})
$$

Solutions of this type are the simplest solutions. In this case the initial equations may be essentially simplified. In (24) derivations may be replaced by multiplication:

$$
\frac{\partial}{\partial t} \rightarrow-i \omega \quad \nabla \equiv \frac{\partial}{\partial \mathbf{r}} \rightarrow i \mathbf{k}
$$

\footnotetext{
${ }^{1}$ The expression for current $\mathbf{j}$ in plasma depends on the model, which is chosen for plasma description (see examples below). If one explores the most complete kinetic consideration, the expression for the plasma current changes and becomes

$$
\mathbf{j}=\sum_{\alpha} e_{\alpha} \int \mathbf{v} d \mathbf{v} f_{\alpha}(\mathbf{v})
$$

where $f_{\alpha}(\mathbf{v})$ is the distribution function for $\alpha$-type plasma particles.
} 
And the initial set reduces to a set of linear algebraic equations:

$$
\begin{array}{ll}
{[\mathbf{k E}]=\frac{\omega}{c} \mathbf{B} ;} & i[\mathbf{k B}]=\frac{4 \pi}{c} \mathbf{j}-i \frac{\omega}{c} \mathbf{E} \\
i \mathbf{k E}=4 \pi \rho ; & \mathbf{k B}=0
\end{array}
$$

Any other, more complicate, solution of the initial equations in linear theory can be presented as a superposition of the simplest solutions with various amplitudes. As follows from the general principle of electrodynamics, this superposition is also a solution of the initial set. This emphasizes the importance of consideration of the solutions in the form of traveling waves.

The motions and continuity equations also may be reduced to algebraic form. Here we present the reduced form of continuity equation only as the motion equations for plasma particles depend on the model, chosen for plasma description (see below):

$$
\omega \rho=\mathbf{k j}
$$

So, the initial equations (consisting of Maxwell's, continuity, and motion equations) reduce to linear algebraic set. The condition for the existence of nonzero solutions of the set is called dispersion relation. It, actually, presents a certain relation between frequency $\omega$ and components of wave vector $\mathbf{k}$. This relation helps determine $\omega$ for the given $\mathbf{k}$ and, vice versa, to determine $\mathbf{k}$ (one of its components) if $\omega$ and other components of $\mathbf{k}$ are given. These statements of the problem are called initial and boundary problems accordingly. These statements are widely used in plasma physics. Herewith in plasma many cases are encountered, in which solution of the initial problem gives complex frequency $\omega=\omega^{\prime}+i \omega^{\prime \prime}$ for real $\mathbf{k}$. In this case the real part of the frequency $\operatorname{Re} \omega=\omega^{\prime}$ shows the frequency of the wave, but the imaginary part shows (depending on its sign) either the increasing of the wave's amplitude if $\omega^{\prime \prime}>0$ or decreasing if $\omega^{\prime \prime}<0$ in accordance to

$$
\exp (-i \omega t)=\exp \left(\omega^{\prime \prime} t\right) \exp \left\{-i \omega^{\prime} t+i \mathbf{k r}\right\}
$$

The solution of the boundary problem should be interpreted in the same manner. If the solution of the problem gives a complex component of the wave vector $\mathbf{k}$, its imaginary part shows either amplification of the given wave in a given direction or its quenching.

\subsection{Electrostatic waves in plasma}

Plasma is a medium, where propagation of specific, electrostatic (or plasma) waves is possible. These waves have no oscillating magnetic field. These waves are also called space charge or Langmuir waves. In the waves electric field is parallel to its propagation direction $\mathbf{k} \| \mathbf{E}$. Oscillations of plasma particles also are parallel to the propagation direction, i.e., the waves are purely longitudinal. These waves play the most important role in plasma and strongly influence on its stability, much more than the usual electromagnetic waves (propagation of which in plasma is also possible).

The explicit expression for plasma conductivity $\sigma$ helps to obtain the dispersion relation for longitudinal waves. When we consider solutions of Maxwell's equations in the form of traveling waves, the conductivity $\sigma$ depends on frequency $\omega$ and wave vector $\mathbf{k}$, i.e., $\sigma \equiv \sigma(\omega, \mathbf{k})$. The dispersion relation for electrostatic waves in plasma can be expressed in terms of $\sigma(\omega, \mathbf{k})$. It has the following form: 


$$
1+i \frac{4 \pi}{\omega} \sigma(\omega, \mathbf{k}) \equiv \varepsilon(\omega, \mathbf{k})=0
$$

where $\varepsilon(\omega, \mathbf{k})$ is the well-known dielectric permittivity of the given media.

Propagation of usual electromagnetic waves in plasma is also possible. The dispersion relation for this case is

$$
k^{2}=\frac{\omega^{2}}{c^{2}} \varepsilon(\omega, \mathbf{k})
$$

In a particular case of vacuum, this expression gives propagation of usual vacuum electromagnetic waves. For vacuum $\varepsilon(\omega, \mathbf{k}) \equiv 1$, and the dispersion relation (37) takes a familiar look, $\omega=k c$.

For further development of the properties of plasma-like media, it is necessary to specify the plasma models.

\section{The simplest plasma models}

Each model of plasma specifies how its particles interact with the electromagnetic field, as well as specifies the behavior of plasma particles inside and between plasma species. Here we consider the simplest models only, leaving aside the most rigorous kinetic consideration. On examples of simple models, we show how the models work as well as some of their advantages and disadvantages.

\subsection{One-particle model}

We begin with the model of one, "average" (or test) particle. In this model particles interact via electromagnetic field, and the interaction, in fact, is weak (it weakly perturbs the motion of the particles). Collisions inside and between species are also taken into account. This model describes the oscillatory properties of gasdischarge and ionosphere plasma well enough. In particular, the model was successfully used for the description of radio-frequency wave propagation through the ionosphere [9].

The initial set of equations in the model of "average" particle includes Newton equations for the "average" electron and for "average" ion along with equations for electromagnetic field and continuity equation:

$$
\begin{aligned}
& \frac{d}{d t} \mathbf{v}_{e}=\frac{e}{m}\left\{\mathbf{E}+\frac{1}{c}\left[\mathbf{v}_{e} \times \mathbf{B}\right]\right\}-\nu_{e n} \mathbf{v}_{e}-\nu_{e i}\left(\mathbf{v}_{e}-\mathbf{v}_{i}\right) \\
& \frac{d}{d t} \mathbf{v}_{i}=\frac{e_{i}}{M}\left\{\mathbf{E}+\frac{1}{c}\left[\mathbf{v}_{i} \times \mathbf{B}\right]\right\}-\nu_{i n} \mathbf{v}_{i}-\nu_{i e}\left(\mathbf{v}_{i}-\mathbf{v}_{e}\right)
\end{aligned}
$$

Here $\mathbf{v}_{e}$ and $\mathbf{v}_{i}$ are the velocities of electrons and ions; $m$ and $M$ are their masses; $\nu_{e n}, \nu_{e i}$ and $\nu_{i n}, \nu_{i e}$ are the frequencies of their collisions, which determine the friction forces inhibiting their motion; $\nu_{e n}$ is the frequency of collisions of electrons with neutral atoms (molecules) and $\nu_{e i}$ with ions, respectively; and for ions, this is $\nu_{i n}$ and $\nu_{i e}$. According to Newton's third law, $m \nu_{e i}=M \nu_{i e}$. A similar system of equations is also used to describe the dynamics of solid-state plasma, but in this case the meaning of the collision frequencies differs from the abovementioned. The frequencies, actually, are the inverse lifetimes of electrons and holes, respectively. 
First we show how this model works on the simplest example of plasma oscillations, as well as how easy the Langmuir frequency follows from this model. Consider pure electron plasma. Ions are heavy and immobile. They serve only for neutralization of electrons' charge. Upon derivation of equations, which describe plasma oscillations, one should recall that we consider linear plasma phenomena. This means that the equations should be linearized, i.e., we consider small perturbations of physical quantities from their basic (equilibrium) state. For example, the density of electrons is considered as $n_{e}=n_{e 0}+n_{e}^{\prime}$ where $n_{e}^{\prime} \ll<n_{e 0}$, and in the resulting expressions we retain the first-order terms only and neglect the terms of second-order smallness (multiplication of the first-order terms). We also take into account that the Langmuir oscillations are potential and use Poisson's equation instead of a full set of Maxwell's equations. For one more simplification, we consider one-dimensional case: let electrons oscillate along the $z$ axis. All this leads to the following: the initial equations (motion, Poisson's, and continuity) are reduced to simple form presented below:

$$
\frac{\partial}{\partial t} \mathrm{v}_{e}^{\prime}=\frac{e E}{m}-\nu_{e i} \mathrm{v}_{e}^{\prime} ; \quad \frac{\partial}{\partial t} n_{e}^{\prime}=-n_{0 e} \frac{\partial}{\partial z} \mathrm{v}_{e}^{\prime} ; \quad \frac{\partial}{\partial z} E=4 \pi e n_{e}^{\prime}
$$

Here $\mathrm{v}_{e}{ }^{\prime}$ is the velocity of electrons, $e$ and $m$ are their charge and mass, $\nu_{e i}$ is the frequency of electron-ion collisions, and $t$ is the time.

For the solutions of (39) that depend on $z$ and $t$ in the form $\exp (-i \omega t+i k z)$, we have the equations

$$
-i\left(\omega+i \nu_{e}\right) \mathrm{v}_{e}^{\prime}=\frac{e E}{m} ; \quad \omega n_{e}^{\prime}-i k n_{0 e} \mathrm{v}_{e}^{\prime}=0 ; \quad i k E=4 \pi e n_{e}^{\prime}
$$

Thus, we arrive to the set of simple algebraic equations, from which the following expression for plasma current results

$$
j=e n_{e}{ }^{\prime} \mathbf{v}_{e}^{\prime}=i \frac{e^{2} n_{0 e}}{m} E \equiv \sigma E, \quad \sigma=i \frac{e^{2} n_{0 e}}{m}
$$

From (41) one can easily obtain the corresponding expression for dielectric permittivity as well as the dispersion relation, which is

$$
\varepsilon=1-\frac{\omega_{p}^{2}}{\omega\left(\omega+i \nu_{e i}\right)}=0
$$

If $\nu_{e i} \ll \omega$ (realizes in most cases), the relation (42) leads to $\omega= \pm \omega_{p}$, i.e., we have free plasma oscillations. If one takes into account plasma collisions, he obtains small negative imaginary correction to the frequency: $\omega_{p} \rightarrow \omega_{p}-i \nu_{e} / 2$. This shows the decay of the oscillations. The decay takes place as a result of collisions.

Obtained results on plasma oscillations and their decay coincide to experimental data. In fact, the model of "average" particle describes plasma well in the considered range of frequencies. Namely this model was used by Langmuir to describe the oscillatory properties of gas-discharge plasma. Also the model was successfully used especially for describing the propagation of radio-frequency waves through the ionosphere [9]. Thereby, the model of "average" particle is justified for highfrequency range. However, in the opposite limit of low frequencies, this model does not lead to reasonable results. That is why, new, more complicate models have been explored. 


\subsection{Two-fluid hydrodynamic: relative electron-ion motion}

The idea to consider plasma as a system consisting of electron and ion fluids arose long time ago. In this model plasma species are described by hydrodynamic equation and interact through the electromagnetic field and through the collisions. The interaction leads to various effects. In particular instability can follow from the interaction. The general theory of plasma instabilities shows that instability is a result of thermodynamically nonequilibrium initial distribution of plasma components.

The initial equations describing electron and ion fluids and their interaction are somewhat more complex than the previous case of "average" particle. They contain additional terms that follow from classical hydrodynamics:

$$
\begin{aligned}
& \frac{d}{d t} \mathbf{v}_{e} \equiv\left[\frac{\partial}{\partial t}+\left(\mathbf{v}_{e} \nabla\right)\right] \mathbf{v}_{e}=-\frac{\nabla\left(n_{e} T_{e}\right)}{m n_{e}}+\frac{e}{m}\left\{\mathbf{E}+\frac{1}{c}\left[\mathbf{v}_{e} \times \mathbf{B}\right]\right\}-\nu_{e n} \mathbf{v}_{e}-\nu_{e i}\left(\mathbf{v}_{e}-\mathbf{v}_{i}\right) \\
& \frac{d}{d t} \mathbf{v}_{i} \equiv\left[\frac{\partial}{\partial t}+\left(\mathbf{v}_{i} \nabla\right)\right] \mathbf{v}_{i}=-\frac{\nabla\left(n_{i} T_{i}\right)}{M n_{i}}+\frac{e_{i}}{M}\left\{\mathbf{E}+\frac{1}{c}\left[\mathbf{v}_{i} \times \mathbf{B}\right]\right\}-\nu_{i n} \mathbf{v}_{i}-\nu_{i e}\left(\mathbf{v}_{i}-\mathbf{v}_{e}\right)
\end{aligned}
$$

Here $m$ and $M$ are electron and ion mass, $T_{e}, T_{i}$, and $n_{e}, n_{i}$ are their temperatures and densities. Other denotations coincide to the previous case of "average" particle above.

The equations describing the electron and ion fluid motion (43) should be supplemented by Maxwell's and continuity equations. Equations for $T_{e}$ and $T_{i}$ (energy balance equations or equations for heat) are also needed. Specific forms of these equations depend on the physical meaning of the problem, which is considered. For simplicity, it may be assumed as $T_{e}, T_{i}=$ const. These assumptions greatly simplify further analysis.

Here we briefly consider a simple example of two-fluid model. In order to show the role of ions and how this role can lead to instability, we consider a case in which electron fluid moves relative to ions in rest. Let $\mathbf{u}$ be the constant velocity of moving electrons. Neutrals are absent. The initial equations of electron and ion fluids (43) and Maxwell's and continuity equations after linearization are reduced to the following set of equations (we assume $T_{e}, T_{i}=0$ and consider the potential oscillation in one-dimensional system and choose the $z$ axis along $\mathbf{u}$ ):

$$
\begin{aligned}
& \left(\frac{\partial}{\partial t}+u \frac{\partial}{\partial z}\right) \mathrm{v}_{e}{ }^{\prime}=\frac{e E}{m}-\nu_{e i} \mathrm{v}_{e}{ }^{\prime} ; \quad \frac{\partial}{\partial t} \mathrm{v}_{i}{ }^{\prime}=\frac{e_{i} E}{M} \\
& \left(\frac{\partial}{\partial t}+u \frac{\partial}{\partial z}\right) n_{e}{ }^{\prime}=-n_{0 e} \frac{\partial}{\partial z} \mathrm{v}_{e}{ }^{\prime} ; \quad \frac{\partial}{\partial t} n_{i}{ }^{\prime}=-n_{0 i} \frac{\partial}{\partial z} \mathrm{v}_{i}{ }^{\prime} \\
& \frac{\partial}{\partial z} E=4 \pi\left(e n_{e}{ }^{\prime}+e_{i} n_{i}{ }^{\prime}\right)
\end{aligned}
$$

Here $\mathrm{v}_{e}{ }^{\prime}$ and $\mathrm{v}_{i}{ }^{\prime}$ are the perturbations of electron and ion fluid velocities accordingly, $n_{e}^{\prime}$ and $n_{i}^{\prime}$ are the perturbations of their densities, $n_{0 e}$ and $n_{0 i}$ are their unperturbed densities, $\nu_{e i}$ is the frequency of electron-ion collisions, and $E$ is the electric field. We look for solutions of the set (44) in the form of waves propagating along the $\mathrm{z}$ axis $\sim \exp (-i \omega t+i k z)$. In this case the equations (44) are reduced to algebraic set. If one performs further steps (determination of induced plasma current, finding plasma conductivity and dielectric permittivity) by analogy to the previous case, he arrives to the expression for dielectric permittivity of considered system, which, in this case, consists of three terms: 


$$
\varepsilon=1+\Delta \varepsilon_{e}+\Delta \varepsilon_{i}
$$

Here the second and third terms are electron and ion contributions in the dielectric permittivity:

$$
\Delta \varepsilon_{e}=-\frac{\omega_{L e}^{2}}{(\omega-k u)\left(\omega-k u+i \nu_{e i}\right)} ; \quad \Delta \varepsilon_{i}=-\frac{\omega_{L i}^{2}}{\omega^{2}}
$$

but the first term is, in fact, the vacuum unit. In (46) $\omega_{L e}=\sqrt{4 \pi e^{2} n_{0 e} / m}$ and $\omega_{L i}=\sqrt{4 \pi e_{i}^{2} n_{0 i} / M}$ are the electron and ion Langmuir frequencies. The dispersion relation of the considered system

$$
1-\frac{\omega_{L e}^{2}}{(\omega-k u)\left(\omega-k u+i \nu_{e i}\right)}-\frac{\omega_{L i}^{2}}{\omega^{2}}=0
$$

is an algebraic equation of the fourth order. Despite the solutions of the fourthorder equations are known (see any reference book on mathematics, e.g., [10]), they are not suitable for our purposes. Very cumbersome expressions with many radicals cannot give physical information, and we choose another way of finding the solutions of (47) and their analysis. The way presented below is typical for plasma theory.

For the analysis we first consider the case of the ion's absence, i.e., if there are streaming electrons only. The dispersion relation is of the second order, with the solution

$$
\omega_{ \pm}=k u \pm \omega_{L e}
$$

This solution represents the waves in the electron stream: fast $(+)$ and slow $(-)$ beam waves. An important fact is that the energy of the slow beam wave is negative [11]. This means that for excitation of the slow wave in the electron beam, one should withdraw energy, but not put energy into the beam. This circumstance plays an important role in theory of streaming instabilities. It lies on the basis of explanation of physical meaning of the most well-known plasma instabilities, beam-plasma instability, as well as the meaning of dissipative beam instabilities. In the last case, dissipation leads not to quenching of the oscillations (as one expects) but to their amplification. The matter is in the following: dissipation serves as a channel for energy withdrawal for excitation of the beam negative-energy wave.

Continuing the analysis of the dispersion relation (47), one can easily see its important peculiarity, which appears as a result of the inequality $\omega_{L i} \ll<\omega_{L e}$; ions play a role under small $\omega$ only:

$$
\omega_{L i} \gg \omega \rightarrow 0
$$

In the opposite case, the contribution of the ions in (47) results in small corrections to the roots that describe the proper oscillations of streaming electron (48). However, if the condition (49) holds, two additional roots of (46) (from a total of four) are approximately equal:

$$
\omega= \pm \frac{\omega_{L i}}{\sqrt{1-\frac{\omega_{L e}^{2}}{k^{2} u^{2}}}}
$$

This expression shows that the instability (caused by the presence of ions) exists if $k u<\omega_{L e}$. In this case the imaginary part of the root (growth rate of unstable oscillations) attains its maximum if $k u \approx \omega_{L e}$. The instability in this case is called resonance 
instability. If the two conditions $\left(\omega \rightarrow 0\right.$ and $\left.k u \approx \omega_{L e}\right)$ are realized, one can ance calculate the roots of the dispersion relation (47), if he rewrites it in the form

$$
\omega\left(\frac{\partial\left(\Delta \varepsilon_{e}\right)}{\partial \omega}\right)_{\omega \rightarrow 0}=\frac{\omega_{L i}^{2}}{\omega^{2}}
$$

From this expression the growth rate of resonant instability follows. It is

$$
\delta_{B n}=\frac{\sqrt{3}}{2^{4 / 3}} \omega_{L e}\left(Z^{2} \frac{m}{M}\right)^{1 / 3}
$$

In the given case, the growth rate is of the same order as the frequency of the unstable oscillations in plasma. This instability was first discovered by Buneman [12]. It (as well as other low-frequency instabilities) plays an important role in many scenarios in space physics and geophysics. The physical essence of this instability lies in the fact that the proper space charge oscillations of moving electrons (beam slow wave) in the frame, associated with the ions in rest due to the Doppler effect, experience red shift, and this greatly reduced frequency becomes close to the proper frequency of ions. Actually the instability is due to the resonance of the negative-energy wave with the ion oscillations.

The models of plasma that have been considered above are very simplified. In spite of this, we have seen that the models describe some phenomena in plasma well enough. For more detailed consideration, one should use the most complete kinetic consideration.

\section{Author details}

Eduard Vardges Rostomyan

Institute of Radiophysics and Electronics, Armenian National Academy of Sciences, Armenia

*Address all correspondence to: eduard_rostomyan@mail.ru

\section{IntechOpen}

(C) 2020 The Author(s). Licensee IntechOpen. This chapter is distributed under the terms of the Creative Commons Attribution License (http://creativecommons.org/licenses/ by/3.0), which permits unrestricted use, distribution, and reproduction in any medium, provided the original work is properly cited. (c) BY 


\section{References}

[1] Fitzpatric R. The Plasma Physics: An Introduction. New York: CRC Press, Taylor \& Francis; 2014

[2] Chen FF. Introduction to Plasma Physics and Controlled Fusion. 2nd ed. New York and London: Plenum Press; 1984

[3] Dendy RO. Plasma Physics. Cambridge, UK: Cambridge University Press; 1995

[4] Bellan PM. Fundamentals of Plasma Physics. Cambridge, UK: Cambridge University Press; 2006

[5] Bittencort JA. Fundamentals of Plasma Physics. NY: Springer; 2004

[6] Silin VP, Rukhadze AA. Electromagnetic Properties of Plasma and Plasma-Like Media (in Russian). Moscow: Atomizdat; 1961

[7] Rukhadze AA. Principles of Plasma Electrodynamics. Berlin: SpringerVerlag; 1984

[8] Lifshits EM, Pipaevskii LP. Physical Kinetics. Amsterdam: Elsevier; 1995

[9] Ginzburg VL. Propagation of Electromagnetic Waves in Plasma (in Russian). Moscow: Nauka; 1967

[10] Korn G, Korn T. Mathematical Handbook. New York: McGraw-Hill Book Company; 1968

[11] Briggs RJ. Electron Stream Interaction with Plasma. Cambridge, Massachusetts: M.I.T. Press; 1964

[12] Buneman O. Instability, turbulence, and conductivity in current-carrying plasma. Physical Review Letters. 1958;1:8 

Section 2

\section{Plasma Applications in Space Technology}





\title{
Chapter 2
}

\section{Hall Thruster: An Electric Propulsion through Plasmas}

\author{
Sukhmander Singh
}

\begin{abstract}
The chapter discussed the technological application of plasma physics in space science. The plasma technology is using laser-plasma fusion, inertial fusion, Terahertz wave generation and welding of metals. In this chapter, the application of plasma physics in the field of electric propulsion and types has been discussed. These devices have much higher exhaust velocities, longer life time, high thrust density than chemical propulsion devices and useful for space missions with regard to the spacecraft station keeping, rephrasing and orbit topping applications. The mathematical relation has been derived to obtain the performance parameters of the propulsion devices.
\end{abstract}

Keywords: electric propulsion, Hall thruster, impulse, exhaust velocity

\section{Overview of propulsion devices and rocket equation}

Electric propulsion (EP) devices use electric power to produce thrust. These devices have much higher exhaust velocities than chemical propulsion devices. Therefore, EP devices require much less propellant mass than chemical systems for a given space task. Here first we have overview of different thrusters and their basic mechanism based on type of propellant used to get the thrust.

The motion of any propulsion devices is given by Newton's 3rd Law of action and equal, opposite reaction which forms the basis for the motivation for the study of electric propulsion. The rocket equation states that a device can accelerate to a desired final velocity by reaction against an expelled propellant stream [1].

Consider a rocket of mass $m$, which expels an infinitely small unit of fuel $d m$ at an exhaust velocity $\vec{U}_{e x}$. The exhaust velocity $\vec{U}_{e x}$ is almost constant and it is a fixed property of the propellant [2]. Conservation of linear momentum requires that the spacecraft experience a small change in velocity $d \vec{v}$, such that

$$
m \frac{d \vec{v}}{d t}+\vec{U}_{e x} \frac{d m}{d t}=0
$$

Integrating by setting appropriate limits in mass and velocity yields

$$
\int_{v_{i}}^{v_{f}} \frac{d \vec{v}}{\vec{U}_{e x}}+\vec{U}_{e x} \int_{m_{i}}^{m_{f}} \frac{d m}{m}=0
$$


After simplification, we get

$$
\ln \left(\frac{m_{f}}{m_{i}}\right)=-\frac{v_{f}-v_{i}}{\vec{U}_{e x}}=-\frac{\Delta \vec{v}}{\vec{U}_{e x}}
$$

The above rocket equation provides the relationship between the mission velocity and the mass of propellant $m_{p}=m_{i}-m_{f}$ required for a given mission. It is clear that a higher $d \vec{v}$ demands more propellant. Unfortunately, the mass ratio cannot be increased so much to avoid payloads problems in space mission, therefore for a given mass fraction, the exhaust velocity $\vec{U}_{\text {ex }}$ of the propellant needs to be the order of $d \vec{v}$ and the higher the propellant exit velocity, the less propellant mass is required.

\section{Thrust, impulse and efficiency}

The performance of thrusters is usually characterized by a number of parameters. A first quantity relevant to thruster performance is the thrust $T$, which is the total force undergone by the rocket. The specific impulse is used to compare the efficiencies of different type of propulsion systems [2]. The performance parameter is the specific impulse $I_{s p}$, defined below

$$
I_{s p}=\frac{T}{\dot{m}_{p} g}
$$

Here $\dot{m}_{p}$ is the mass flow rate and $g$ is the acceleration due to gravity. The specific impulse has the dimension of time and is a measure for the effective lifetime of the thruster, when lifting its own propellant from the earth's surface.

For the case of a constant mass flow rate the thrust is also constant as

$$
T=\dot{m}_{p} \vec{U}_{e x}
$$

and the specific impulse simplifies to

$$
I_{s p}=\frac{\vec{U}_{e x}}{g}
$$

Finally, the rocket equation turned into

$$
\frac{m_{f}}{m_{i}}=e^{\frac{-\Delta \vec{v}}{g_{s p}}}
$$

The rocket equation is equally applicable to all type of propulsion systems. Therefore high specific impulse related to better efficiency for a propellant. Based on the acceleration of gases for propulsion, electrical thrusters have been classified into three main categories.

\subsection{Electrothermal thrusters}

In electrothermal thrusters, the hot gas is expanded through a nozzle without ionizing it. When it is being passed through a thin nozzle, the thermal energy of gas gets converted into kinetic energy and produce a thrust. 


\subsection{Electromagnetic thrusters}

In electromagnetic thrusters an inert gas is used as a propellant and it is ionized by heating to produce plasma. Then these ionized gas (charged particles) are acceleration by electromagnetic force to generate thrust.

\subsection{Electrostatic thrusters}

In electrostatic thrusters only ions are accelerated by applying direct electric field at the exit side of the thruster to produce thrust.

\section{Hall thruster operation}

Hall effect thrusters (HETs) were originally developed in United States and Russia about 60 years ago, and the first working devices were reported in U.S. in the early 1960s. Now a days, most of the countries using the Hall thruster technology in their space mission. Unlike chemicals and electric rockets, the propulsive thrust in a Hall thruster is achieved by an ionized inert gas (Xenon) which has high atomic number and low ionization potential. For this Xenon is mostly used. In a Hall thruster, the propellant is ionized and then accelerated by electrostatic forces.

Figure 1 shows the internal parts of a plasma Hall thruster. Generally, the discharge channel is cylindrical shape made up with metallic material. The magnetic field of the order of 150 Gauss is applied to produce closed drift of electrons inside the channel. The applied magnetic field which is strong enough so that the electrons get magnetized, i.e. they are able to gyrate within the discharge channel, but the ions remain unaffected due to their Larmor radius much larger than the dimension of the thruster. Thus the electrons remain effectively trapped in azimuthally $\vec{E} \times \vec{B}$ drifts around the annular channel and slowly diffuse towards the anode. This azimuthal drift current of the electrons is referred to as the Hall current. The propellant enters from the left side of the channel via anode and gets ionized through hollow cathode of the device. The electric field of strength $\sim 1000 \mathrm{~V} / \mathrm{m}$ gets

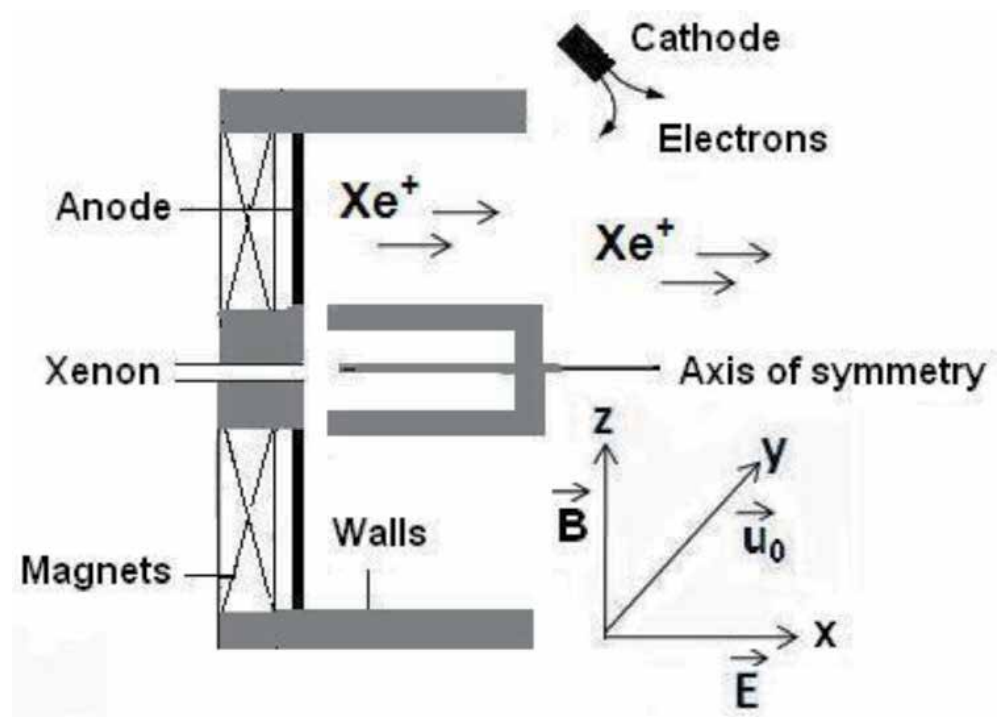

Figure 1.

Schematic diagram of a typical Hall plasma thruster. 
generated inside the discharge channel along the axial direction of the device [3]. In addition, these kind of devices have implication in partially ionized plasmas (tokamaks), in ionosphere (base of the solar photosphere), in protoplanetary discs, circum nuclear discs in active galactic nuclei and neutron stars. Hall thruster has high thrust resolution, it is being used for the adjustment of the location of the satellite onboard.

\section{Spacecraft issues}

The first issue is that the divergence angle of these devices is about $60^{\circ}$, which relatively large and cause problem related to erosion of the channel walls and outer surfaces of the thruster. The erosion of the walls decreases the lifetime of the device. This channel usually has a length of the order of centimeters. In addition, densities in the channel are typically in the range between $10^{17}$ and $10^{18} \mathrm{~m}^{-3}$ for the plasma, and $10^{18}$ and $10^{20} \mathrm{~m}^{-3}$ for the neutral gas [4]. The plasma in a Hall thruster does not stay uniform and an inhomogeneous plasma immersed in the external electric and magnetic fields is not in the thermodynamically equilibrium state, this deviation in general is a source of plasma instabilities. The amplitudes of the waves and instabilities are attributed by the density scale lengths of plasma and magnetic field and other parameters. These waves/oscillation and instabilities may affect the efficiency of the device, hence forth research on studies on oscillation/instabilities always attracted the investigators.

\section{Types of Hall plasma thruster}

Two types of Hall thrusters have been developed: a thruster with closed electron drift and extended acceleration zone or stationary plasma thruster and a thruster with a very short acceleration channel or thruster with anode layer.

In Table 1, typical values of some of the pertinent properties are listed at the thruster exit for the SPT-100.

\subsection{Dielectric wall thruster or stationary plasma thruster}

Such thrusters have a wall made up of dielectric of boron nitride or silicon carbide and extended channel compared to its width. The role of the wall is that the collisions of the electrons and ions with the wall generate low energy secondary electrons. These secondary electrons keep tending the electron temperature low in the discharge plasma. By reducing the discharge electron energy, a smooth and continuous variation in plasma potential between the anode and the cathode is

\begin{tabular}{lccc}
\hline Property & Value & Property & Value \\
\hline Inner diameter & $60 \mathrm{~mm}$ & Neutral velocity & $\sim 300 \mathrm{~m} / \mathrm{s}$ \\
\hline Outer diameter & $100 \mathrm{~mm}$ & Electron temperature & $5-10 \mathrm{eV}$ \\
\hline Plasma density & $\sim 10^{17} / \mathrm{m}^{3}$ & Ion temperature & $1-5 \mathrm{eV}$ \\
\hline Neutral density & $\sim 10^{18} / \mathrm{m}^{3}$ & Neutral temperature & $0.9 \mathrm{eV}$ \\
\hline Ion velocity & $\sim 10^{4} \mathrm{~m} / \mathrm{s}$ & Debye length & $\sim 10^{-5} \mathrm{~m}$ \\
\hline Collision mean free & $\sim 1 \mathrm{~m}$ & & \\
\hline
\end{tabular}

Table 1.

Typical plasma parameters for Hall Thrusters. 
obtained. Since the dielectric walls are not conductive, charge builds up along the length of the acceleration channel that leads to a variable potential profile along its length.

\subsection{Thruster with anode layer}

Thruster with anode layer also developed in Russia has a narrow acceleration zone associated with the narrow electric field region near the anode. This geometry considerably shortens the electric field region in the channel, where the ion acceleration occurs. However, this configuration does not change the basic ion generation or acceleration method. The channel wall made up of conductor, which is usually also a part of the magnetic circuit, is biased negatively (usually cathode potential) to repel electrons in the ionization region and to reduce electron-power losses. This reduces the loss caused by the ion and electron collisions with the walls. Since the walls are conductive, a constant potential (same as that of the cathode) is observed along the entire wall. Very high electron temperatures, i.e. more than $50 \mathrm{eV}$, are typically observed in such thrusters [1].

\section{Review of status of current research and development in the subject}

The range of the oscillations lies from few $\mathrm{kHz}$ to $\mathrm{MHz}$ in the acceleration channel of the thrusters and has been given in Table 2. Rayleigh-Taylor (RT) instability takes place when a lighter fluid supports a heavy fluid. The plasma in the Hall thruster possesses Rayleigh-Taylor instability, resistive instability, transit time instability, electromagnetic instability and sheath instabilities [5-11]. These systems are rampant with plasma instabilities and fluctuations, many of which are responsible for performance, driving electron transport across magnetic field lines and contributing to propellant ionization. Over the last decade several studies have been carried out with HET to characterize the low frequency azimuthal and axial oscillations and optimizing magnetic field profile for a wide range of operating conditions for better efficiency and performance. Singh and Malik [10,11], investigated that temperature of the ion and drift velocity profiles of the electron modifies the conditions for Rayleigh type instability under the effects of thermal motions of ions.

The plasma resistivity induces resistive instabilities (electrostatic and electromagnetic) [6-9] associated with azimuthal and axial directions. High-frequency (1-10 MHz) instabilities have been studied in the Hall-effect thruster [6-9], where it was found that these instabilities have the highest level near the thruster exit plane. These oscillations in the Hall thruster determine the efficiency of the system and may affect the divergence of the ion beam and electron transport across the

\begin{tabular}{lll}
\hline Range $(\mathbf{k H z})$ & Type & Driving mechanism \\
\hline $10-20$ & Loop or circuit oscillations & $\begin{array}{l}\text { Magnetic field, discharge voltage and electron } \\
\text { wall collision frequency }\end{array}$ \\
\hline $5-25$ & Rotating spokes & Ionization process \\
\hline $20-60$ & Azimuthal modes or drift instability & Gradient of density and magnetic field \\
\hline $70-500$ & Transient time oscillations & Plasma density gradient and low ionization \\
\hline $0.5-5 \mathrm{MHz}$ & Azimuthal waves & Drift velocity of plasma species \\
\hline
\end{tabular}

Table 2.

Range and classification of oscillations in a Hall Thrusters. 
magnetic field. Smolyakov et al. reported that sheath instabilities has a vital role in anomalous transport phenomena in Hall plasma thruster [12, 13].

\section{Ion stream speed study for electrostatic thruster}

Let us consider a Hall thruster having potential difference between anode and virtual cathode is $\Phi$ Volt and ions density (mass) $n(M)$. The mass flow of propellant of ions of mass $M$ through an area $A$ is given by $\dot{m}_{p}=n M A \vec{U}_{e x}$.

The thrust is also constant as

$$
T=\dot{m}_{p} \vec{U}_{e x}
$$

Substituting the value of mass flow rate, the thrust per unit area

$$
\frac{T}{A}=\left(n M \vec{U}_{e x}\right) \vec{U}_{e x}=\frac{J_{i}}{q} M \vec{U}_{e x}
$$

where $J_{i}$ the current density of ions.

From the definition of work energy theorem, that the kinetic energy of each ion should equal to the work done in moving the charge across a potential drop. That is

$$
\frac{1}{2} M \vec{U}_{e x}^{2}=q \Phi
$$

Or

$$
\vec{U}_{e x}=I_{s p} g=\sqrt{\frac{2 q \Phi}{M}}
$$

Thus the specific impulse or exhaust velocity of the ions depends on the potential drop developed across the anode and cathode and to the mass of the ion.

\section{Conclusions}

The $\vec{E} \times \vec{B}$ configurations of fields are used to confine electrons, increasing the electron residence time and allowing ionization and plasma sustainment. The magnetron sputtering used in material science for ion implantation is also based on the same $\vec{E} \times \vec{B}$ drift. The primary concern of the study to enhance the lifetime and performance of the Hall thruster by studying the instabilities that takes place in the channel and optimization of profile of the magnetic field which is the main parameter in respect to the erosion of the channel walls.

\section{Acknowledgements}

The University Grants Commission (UGC), New Delhi, India is thankfully acknowledged for providing the startup Grant (No. F. 30-356/2017/BSR). 


\section{Author details}

Sukhmander Singh

Plasma Waves and Electric Propulsion Laboratory, Department of Physics, Central University of Rajasthan, Ajmer, Kishangarh, India

*Address all correspondence to: sukhmandersingh@curaj.ac.in

\section{IntechOpen}

(C) 2020 The Author(s). Licensee IntechOpen. This chapter is distributed under the terms of the Creative Commons Attribution License (http://creativecommons.org/licenses/ by/3.0), which permits unrestricted use, distribution, and reproduction in any medium, provided the original work is properly cited. (c) BY 


\section{References}

[1] Kaufman HR. Technology of closeddrift thrusters. AIAA Journal. 2012; 23(1):78-86. DOI: $10.2514 / 3.8874$

[2] Goebel DM, Katz I. Fundamentals of Electric Propulsion: Ion and Hall Thrusters. New York: Wiley; 2008

[3] Jahn RG. Physics of Electric Propulsion. New York: McGraw-Hill; 1968

[4] Martinez-Sanchez M, Pollard JE. Spacecraft electric propulsion: An overview. Journal Propulsion Power. 1998;14(5):688-699

[5] Choueiri EY. Plasma oscillations in hall thrusters. Physics of Plasmas. 2001; 8(4):1411-1426

[6] Singh S, Malik HK, Nishida Y. High frequency electromagnetic resistive instability in a hall thruster under the effect of ionization. Physics of Plasmas. 2013;20:102-109 (1-7)

[7] Singh S, Malik HK. Growth of low frequency electrostatic and electromagnetic instabilities in a hall thruster. IEEE Transactions on Plasma Science. 2011;39:1910-1918

[8] Singh S, Malik HK. Resistive instabilities in a hall thruster under the presence of collisions and thermal motion of electrons. The Open Plasma Physics Journal. 2011;4:16-23

[9] Malik HK, Singh S. Resistive instability in a hall plasma discharge under ionization effect. Physics of Plasmas. 2013;20:052115 (1-8)

[10] Singh S, Malik HK. Role of ionization and electron drift velocity profile to Rayleigh instability in a hall thruster plasma: Cutoff frequency of oscillations. Journal of Applied Physics. 2012;112:013307 (1-7)
[11] Malik HK, Singh S. Conditions and growth rate of Rayleigh instability in a hall thruster under the effect of ion temperature. Physical Review E. 2011; 83:036406 (1-8)

[12] Nejoh Y, Yamamura Y. Simulation of the collisional sheath structure near the outer wall of the hall thruster. Physics of Plasmas. 2005;12:033506

[13] Smolyakov AI, Frias W, Kaganovich ID, Raitses Y. Sheathinduced instabilities in plasmas with $\mathrm{E} \times \mathrm{B}$ drift. Physical Review Letters. 2013;111:115002 
Section 3

\section{Microwave Plasma Interactions}





\title{
Evolution of Microwave Electric Field on Power Coupling to Plasma during Ignition Phase
}

\author{
Chinmoy Mallick, Mainak Bandyopadhyay and Rajesh Kumar
}

\begin{abstract}
During the gas ignition process, the plasma and the microwave electric fields are evolved with time together in the plasma volume. The spatio-temporal evolution pattern of microwave-radiated plasma parameters is reported here, highlighting the role of these electric fields on power coupling processes. Evolutions of electric field and so power coupling processes are calculated using the finite element method (FEM). It is observed that the main power coupling mechanism is electron cyclotron resonance (ECR) method; however, with the evolution of plasma, the mode shifts from ECR to off-ECR-type heating with time. Off-ECR heating in the form of upper hybrid resonance (UHR) method, electrostatic (ES) ion acoustic wave heating method is important heating mechanisms during highly dense plasma condition, when density is above critical density for launched frequency, $2.45 \mathrm{GHz}$. The conclusions on the shifting of heating mechanisms are also drawn based on the 3D maps of spatio-temporal plasma density and hot electron temperature evolution.
\end{abstract}

Keywords: microwave plasma simulation, COMSOL multi-physics, magnetized plasma evolution, ECR, off-resonance, ion source, electrostatic heating, electric field evolution, hot electron temperature, experimental validation

\section{Introduction}

Gaseous particles are ionized to bring them in the form of plasma through the various heating techniques. One of the popular heating techniques is the injection of high frequency microwaves (MW) to a cylindrical cavity that has comparable dimension to the injected MW wavelength. The MW plasma generated by the continuous or pulse feeding of the MW is used in the applications of industrial and accelerator fields for the material science and nuclear applications, respectively. In both of the feeding cases, the plasma is basically produced due to the power absorption by the electrons from the space-time dependent electric field of the MW. The spatio-temporal dynamics and also the steady-state behaviors of the plasma are governed by the ways the MW are coupled to the plasma sustained inside a cavity. The behavioral pattern of the electric field during the plasma evolution can help us to comment on the different MW coupling ways/mechanisms that are involved in the formation of plasma particles and their confinement scenarios. By mastering the basic concepts on those different coupling mechanisms, the coupling efficiency and so the performance of that particular plasma source can be optimized. Performance 
optimization for this kind of plasma source is indispensable as these are involved in various kinds of applications as mentioned above. One of the important plasma devices is the microwave ion sources that are operated in continuous as well as pulse mode to extract the ion beam during the transient and steady state periods of plasma loading conditions [1-5]. The beam qualities are influenced by the MW coupling mechanisms as they are involved in deciding the plasma parameters during the extraction of a particular instant of the plasma evolution time. Several studies have already reported the electric field evolution during few $10 \mathrm{~s}$ of microsecond range when the plasma density was increasing in the very similar plasma device. The electric field was dropped by about more than $50 \%$ within a span of few microseconds after the MW launch ( $\mathrm{t}=0 \mathrm{~s})$ into the cavity [6-11].

Since the electric fields can affect the different power coupling mechanisms during the gas ignition moment (ns to $\mu \mathrm{s}$ ), the spatio-temporal plasma parameters are influenced significantly especially in the low pressure regime. Many researchers have used the kinetic models like PIC/MCC or even the hybrid fluid/PIC to obtain more precise results in the MW plasma discharge. But they failed to estimate the hot electron dynamics efficiently in lower pressure condition, as these models demand intensive computational hardware due to its particle approach. Therefore, the current chapter presents the electric field evolution and its impact on the plasma parameter build-up during low pressure plasma state. Here, the model used is based on the finite element method (FEM) that gives more appropriate results for the transient plasma parameters through fluid modeling approach and time-dependent, partial differential equation solver (TDPDE) using fewer computer resources [11]. The different MWplasma coupling mechanisms (ECR, UHR and electric field polarity reversal associated with ES wave heating) during the plasma density evolution after the MW launch $(t=0 \mathrm{~s})$ can be understood from the behaviors of electric fields.

\section{Basic theory of microwave plasma interaction}

The study on the propagation and interactions of the microwave with the plasma is important to optimize the performance of any plasma devices like the microwave ion sources. The microwave propagation in the plasma is affected by the dielectric prosperities of the plasma medium. The dielectric property, i.e., the permittivity or the refractive index of the plasma, depends on the external magnetic field distribution that is used to confine the plasma particles and also the electrostatic fields that are present in the plasma. Therefore, the microwave while propagating in different directions within the plasma encounters different values of the refractive index as well as the permittivity that makes the magnetized plasma to be anisotropic and inhomogeneous, respectively. To generate a high plasma density, which is one of the primary requirements in some microwave plasma devices, viz., the microwave discharge ion source (MDIS) or electron cyclotron resonance ion sources (ECRIS), an optimum coupling of the microwave energy through the different interaction mechanisms to the plasma medium is necessary. The microwave propagation and the coupling mechanisms are also influenced by the boundary conditions present in the plasma devices and the geometrical shape of the plasma device. In most cases, the dimension of the plasma reactor used for the purpose of ion sources lie in the comparable range of the launched microwave wavelength. This means the microwave electromagnetic field propagation within the ion source reactor (or cavity) is guided by the boundary conditions and the geometrical shape of the ion source cavity. So, the microwave electromagnetic field coupling to the plasma is affected if the cavity geometry is perturbed. Due to the modification of the cavity geometry, the resonating properties of the cavity resonator are no longer dominated by the 
fundamental cavity resonant mode. The cavity can resonate with some additional resonating frequencies including the fundamental one. The additional resonating frequencies can lie near to the fundamental one. Due to this reason, if the microwave is launched to the modified cavity, the total microwave field is shared among the cavity resonant modes including the fundamental one and contributes to the power coupling to the plasma.

From the electromagnetic theory of a resonant cavity, only particular cavity resonant modes can exist having fixed frequencies that are given by $[12,13]$

$$
f=\frac{1}{2 \pi} \sqrt{\frac{r^{2} \pi^{2}}{l^{2}}+h^{2}}
$$

where $r, l$ and $h$ are the integer, the length of the plasma cavity and the eigenvalues of the cavity, respectively. The eigenvalues are obtained from the solution of the equations gives by:

$$
\begin{gathered}
\nabla_{t}^{2} H_{z 0}+h^{2} H_{z 0}=0 \\
\nabla_{t}^{2} E_{z 0}+h^{2} E_{z 0}
\end{gathered}
$$

A theoretical calculation for the cavity resonant modes from the eigenvalue equations for the electromagnetic field $[12,13]$ is performed from the empty and completely closed cavity. By considering a simplest cylindrical cavity of radius $r$ and the length ' $d$ ' that is filled with a dielectric constant $\varepsilon_{r}$ and the relative permittivity $\mu_{r}$, the resonant frequencies allowed by the cavity that can be determined from the eigenvalue equations are shown below from the TE and TM modes:

$$
\begin{aligned}
& f_{n m l}^{T E}=\frac{c}{2 \pi \sqrt{\mu_{r} \varepsilon_{r}}} \sqrt{\left(\frac{P_{n m}^{\prime}}{r}\right)^{2}+\left(\frac{l \pi}{d}\right)^{2}} \\
& f_{n m l}^{T E}=\frac{c}{2 \pi \sqrt{\mu_{r} \varepsilon_{r}}} \sqrt{\left(\frac{P_{n m}}{r}\right)^{2}+\left(\frac{l \pi}{d}\right)^{2}}
\end{aligned}
$$

Here, $P_{n m}$ and $P_{n m}^{\prime}$ are, respectively, the zeros of order $\mathrm{m}$ of the Bessel functions of order $\mathrm{n}$ and its first derivative. The indices represent the three-dimensional electromagnetic field patterns for each cavity resonant mode.

\subsection{Microwave propagation in plasmas}

In case of magnetized plasma, the microwave propagation is influenced by the plasma particle dynamics. As the plasma particle dynamics are represented by the particle velocity, the thermal velocity of the plasma particles should be considered when the microwave propagation in plasma is discussed. If the thermal velocity of the plasma particles is negligible with respect to the phase velocity of the microwave, i.e., $v_{\text {thermal }} \ll v_{\text {phase }}$, this approximation is useful for determining the dispersion of the microwave in a magnetized plasma. This approximation also known as 'cold plasma' is not applicable to the locations where the microwave encounters resonance in the magnetized plasma. On the other hand, if the temperature of the plasma particles is high enough that makes the velocity of the particle to be relativistic (also called as 'warm plasma'), the influence of the plasma particles cannot be neglected while determining the microwave propagation. In that case, the damping of the microwave field is greatly influenced by the plasma particles. 
For the un-magnetized plasma case in which the plasma is considered to be isotropic and the condition, $v_{\text {thermal }} \ll v_{\text {phase }}$ is satisfied, the plasma fluid equation is written as:

$$
\frac{\partial \tilde{v}}{\partial t}=q \tilde{E}
$$

If the microwave electric field $(\tilde{E})$ and the velocity $(\tilde{v})$ are assumed to be varying with $e^{i \omega t}$, the plasma dielectric constant can be easily shown as:

$$
\epsilon=\epsilon_{0}\left(1-\frac{\omega_{p e}^{2}}{\omega^{2}}\right)
$$

where the $\omega_{p e}$ is the electron plasma frequency that is also obtained from the electron density by the relation:

$$
\omega_{p e}=\sqrt{\frac{n_{e} e^{2}}{\epsilon_{0} m_{e}}}
$$

where $m_{e}$, e and $\epsilon_{0}$ are the electron mass, charge and electrical permittivity in vacuum condition, respectively. So the electrical permittivity in plasma is affected by the plasma density. As the electrical permittivity has to be positive in ideal case, the plasma density has to be lower than the corresponding microwave frequency that is launched externally to energize the plasma particles. Therefore, in case of homogeneous and isotropic plasma, it is not possible to raise the plasma density beyond certain level that is known as the critical density, which is written as $n_{\text {critical }}=\omega_{p e}^{2}\left(\frac{\epsilon_{0} m_{e}}{n_{e} e^{2}}\right)$. The density below and above the critical density is referred to be underdense and overdense plasma. So the critical density is the main limitation in the un-magnetized plasma for the microwave propagation. The dispersion for the wave in magnetized plasma is written as:

$$
\omega^{2}-\omega_{p e}^{2}=k^{2} c^{2}
$$

In the overdense plasma, if $\omega<\omega_{p e}$, the propagation $k$-vector ' $k$ ' becomes imaginary that means the complete reflection of the incoming microwave from the plasma. Therefore, if the microwave propagation is assumed to be propagating in the $x$-direction in the overdense plasma, the electric field of the microwave can be written as:

$$
\tilde{E}=\tilde{E}_{0} e^{(i k . \tilde{r}-\omega t)}=\tilde{E}_{0} e^{(i k x-\omega t)}=e^{\frac{x}{\delta}} e^{-i \omega t}
$$

So, before encountering the overdense plasma, the electric field becomes an evanescent wave as its magnitude decays exponentially within a distance of approximately the skin depth value; $(\delta)=c /\left(\sqrt{\omega_{p e}^{2}-\omega^{2}}\right)$.

Under the externally applied magnetic field, the dielectric constant for the anisotropic plasma becomes a tensor quantity. It means the microwave propagation becomes dependent on the plasma dielectric properties while propagating in various directions with respect to the externally applied magnetic field. If the magnetic field is oriented axially $\left(\tilde{B}=B_{0} \tilde{z}\right)$ in a cylindrical plasma chamber, the plasma can act as a dielectric with current $\tilde{J}$ and also the Maxwell's equations can be rewritten as: 


$$
\tilde{\nabla} \times \tilde{B}=\mu_{0}\left(\tilde{J}+\varepsilon_{0} \frac{\partial \tilde{E}}{\partial t}\right)=\mu_{0} \frac{\partial \tilde{D}}{\partial t}
$$

Now if the plasma motion follows the $e^{i \omega t}$ dependence and the plasma conductivity tensor is related with the current as $\overline{\bar{\sigma}} . \tilde{E}=\tilde{J}$, the relation between $\tilde{D}$ and $\tilde{E}$ is obtained as:

$$
\tilde{D}=\varepsilon_{0}\left(\overline{\bar{I}}+\frac{i}{\varepsilon_{0} \omega} \overline{\bar{\sigma}}\right) \tilde{E}
$$

where the symbol $\overline{\bar{I}}$ represents an identity tensor. In short, the parameter $\tilde{D}$ can also be written as $\tilde{D}=\varepsilon_{0} \overline{\overline{\epsilon_{r}}} \tilde{E}$, where the effective dielectric tensor $\overline{\bar{\epsilon}}$ is represented as $\epsilon_{0}\left(\overline{\bar{I}}+\frac{i}{\varepsilon_{0} \omega} \overline{\bar{\sigma}}\right)$. The plasma conductivity tensor is evaluated from the fluid plasma approach. Therefore, the fluid equation in case of 'cold plasma' approximation is rewritten by neglecting the collisional and pressure term and the magnetic field term is considered as:

$$
m_{i} \frac{\partial \tilde{v}}{\partial t}=e(\tilde{E}+\tilde{v} \times \tilde{B})
$$

The solution to this equation brings out the relation between the velocity $(\tilde{v})$ and the electric field of the microwave. As $\tilde{J}=n e \tilde{v}$, the final expression for the effective dielectric tensor $\overline{\bar{\epsilon}}$ can be shown in a determinant form as:

$$
\overline{\bar{\epsilon}}=\epsilon_{0}\left|\begin{array}{ccc}
S & -i D & 0 \\
i D & S & 0 \\
0 & 0 & P
\end{array}\right| \equiv \epsilon_{0} \overline{\overline{\epsilon_{r}}}
$$

where $S, D$ and $P$ can be written in terms of three different kinds of frequencies: $S=\left[1-\frac{\omega_{p e}^{2}}{\omega^{2}}\left(\frac{1}{1-\omega_{c}}\right)\right], D=\left[-\frac{\omega_{p e}^{2}}{\omega^{2}} \frac{\omega_{c}}{\omega}\left(\frac{1}{1-\omega_{c}}\right)\right]$ and $P=1-\frac{\omega_{p e}^{2}}{\omega^{2}}$.

The wave equation can be derived from the Maxwell's curl equation for the electric field by following the standard procedure that shows

$$
\tilde{\nabla} \times \tilde{\nabla} \times \tilde{E}=-\frac{\partial \tilde{\nabla} \times \tilde{B}}{\partial t}=-\frac{1}{c^{2}} \overline{\bar{\epsilon}} \cdot \frac{\partial^{2} \tilde{E}}{\partial t^{2}}
$$

The above equation is rewritten by assuming the $e^{i \tilde{k} . \tilde{r}}$ dependence of the electric field as:

$$
\tilde{k} \times \tilde{k} \times \tilde{E}+\frac{\omega^{2}}{c^{2}} \overline{\bar{\epsilon}} \cdot \tilde{E}=0
$$

The equation can be represented in matrix form by assuming the angle between the wave vector and magnetic field to be ' $\theta$ ' and denoting the vector $\tilde{N}=\frac{c}{\omega} \tilde{k}$.

$$
\left[\begin{array}{ccc}
S-N^{2} \cos ^{2} \theta & -i D & N^{2} \cos \theta \sin \theta \\
i D & S-N^{2} & 0 \\
N^{2} \cos \theta \sin \theta & 0 & P-N^{2} \sin ^{2} \theta
\end{array}\right]\left[\begin{array}{c}
E_{x} \\
E_{y} \\
E_{z}
\end{array}\right]=0
$$


The solution to these equations can exist if the determinant is zero. This condition brings out the dispersion relation of the microwave in the plasma. By making the determinant to be zero, two solutions are obtained that are written as:

$$
N_{O, X}^{2}=\frac{2 x(1-x)}{2(1-x)-y^{2} \sin ^{2} \theta \pm \sqrt{y^{4} \sin ^{4} \theta \pm 4 y^{2}(1-x)^{2} \cos ^{2} \theta}}
$$

where the symbols ' $O$ ' and ' $X$ ' correspond to the ordinary microwave and extraordinary microwave, respectively. The notations $x$ and $y$ in Eq. (18) represent the electron density scale and magnetic field scale respectively and are denoted as: $x=\frac{\omega_{p e}^{2}}{\omega^{2}} \propto n_{e}$ and $y=\frac{\omega_{c}}{\omega} \propto B_{0}$.

From the solutions, the microwave propagation and damping properties can be explained considering the values of the electron density, magnetic field and the angle of propagation. By the definition, the refractive index $(N)$ has to be positive for enabling the microwave to propagate in plasma. The two different values for the refractive index $N=0$ and $N=\infty$ imply the cut-off and the resonance conditions for the microwave, respectively. From Eq. (18), three different types of cut-offs can be obtained by putting $N=0$ that depends on the plasma parameters. Three cut-offs are:

(1) $\omega=\omega_{p e}$; this cut-off is also known as 'O' cut-off; (2) $\omega=\omega_{R}=$

$0.5 \sqrt{\omega_{c}^{2}+4 \omega_{p e}^{2}}+\omega_{c}$; this cut-off is also called upper or R cut-off frequency that occurs both above $\omega_{p e}$ and $\omega_{c}$; and (3) $\omega=\omega_{L}=0.5 \sqrt{\omega_{c}^{2}+4 \omega_{p e}^{2}}-\omega_{c}$; it is also called the lower cut-off or L cut-off for the microwave. Based on the local plasma density values and magnetic field values in the plasma volume, "ordinary waves" have ' $O$ ' cut-off region, and extra-ordinary waves have two cut-offs, R cut-off, and L cut-off regions, from where corresponding waves are reflected back. To satisfy resonance condition $(N=\infty)$, it is found from Eq. (18) that the term $P \cos ^{2} \theta+S \sin ^{2} \theta$ has to be zero. It gives the resonance angle in terms of the plasma parameters that are given by:

$$
\cos ^{2} \theta=\frac{x+y^{2}-1}{x y^{2}}
$$

It is clear that the wave can resonate at the resonance angle $(\theta)$ that is decided by the angle of microwave propagation with respect to the magnetic field. From Eq. (18), it is also confirmed that only the extraordinary wave can have the resonances. From Eq. (19), the resonance angles for the extraordinary wave are determined by the plasma density and the magnetic field. For example, if the resonance angle is set to be zero, it is verified that the resonance takes place at $\omega=\omega_{R F}=\omega_{c}$. This means the microwave matches the larmour frequency of the electrons that are gyrating around the magnetic field lines. It is also known as electron cyclotron resonance (ECR). Again, for the resonance angle, $\theta=90^{\circ}$, another type of resonance can occur when $x+y^{2}-1=0$, and this condition yields the resonance frequency to be $\omega=\omega_{R F}=\sqrt{\omega_{c}^{2}+\omega_{p e}^{2}}$. This type of resonance is known as upper hybrid resonance (UHR) applicable to the wave (e.g., extraordinary wave) that is propagating perpendicularly with respect to the magnetic field. The resonance of the ordinary wave can also be understood from Eq. (18). It can be seen from one of the solutions obtained from Eq. (19) that another resonance can exist in the region where $x, y \geq 1$. This is the forbidden region for the ordinary wave, which is the ' $O$ ' cut-off region. Therefore, the ordinary-type microwave can reach this resonance only by tunneling through this cut-off region. 
To visualize the cut-offs and resonances for the different types of microwaves in a better way, the dispersion plots are shown in a single diagram, also known as Clemmow-Mullaly-Allis (CMA) diagram. Figure $\mathbf{1}$ is applicable to the 'cold plasma' approximation case as discussed before. In the CMA diagram, the $x$ - and $y$-axes represent the plasma density and the magnetic field, respectively. It means that for a given launch microwave frequency, a point on the diagram dictates the experimental situation that is characterized by a particular plasma density and the magnetic field. The diagram is divided into two regions: underdense and overdense plasma regions that are based upon the launch microwave frequency. The boundaries shown in the diagram correspond to the cut-off and resonances for the different types of microwaves.

The polarization of the electric field of the microwaves also plays important role on the damping of microwave power into the plasma. From Eq. (17), the relation between the $x$ - and $y$-components of the electric field can be found out to understand the polarization in the plane perpendicular to the magnetic field. The relation is obtained from Eq. (17) as:

$$
\frac{i E_{x}}{E_{y}}=\frac{N^{2}-S}{D}
$$

Eq. (20) indicates that the waves become circularly polarized and linearly polarized when the cut-off condition $\left(N^{2}=0\right)$ and the resonance condition $\left(N^{2}=\infty\right)$ are satisfied respectively. The waves are circularly and linearly polarized, respectively. However, it is confirmed that the ordinary waves are left hand polarized and the extraordinary waves are right hand polarized. The electric field of the right hand polarized waves and the electrons gyrating across the magnetic rotate in the same direction. Therefore, if the microwave-launched frequency matches the electron gyration frequency, electrons observe a constant electric field at the resonance location and are resonantly accelerated by the electric field. It is known as electron

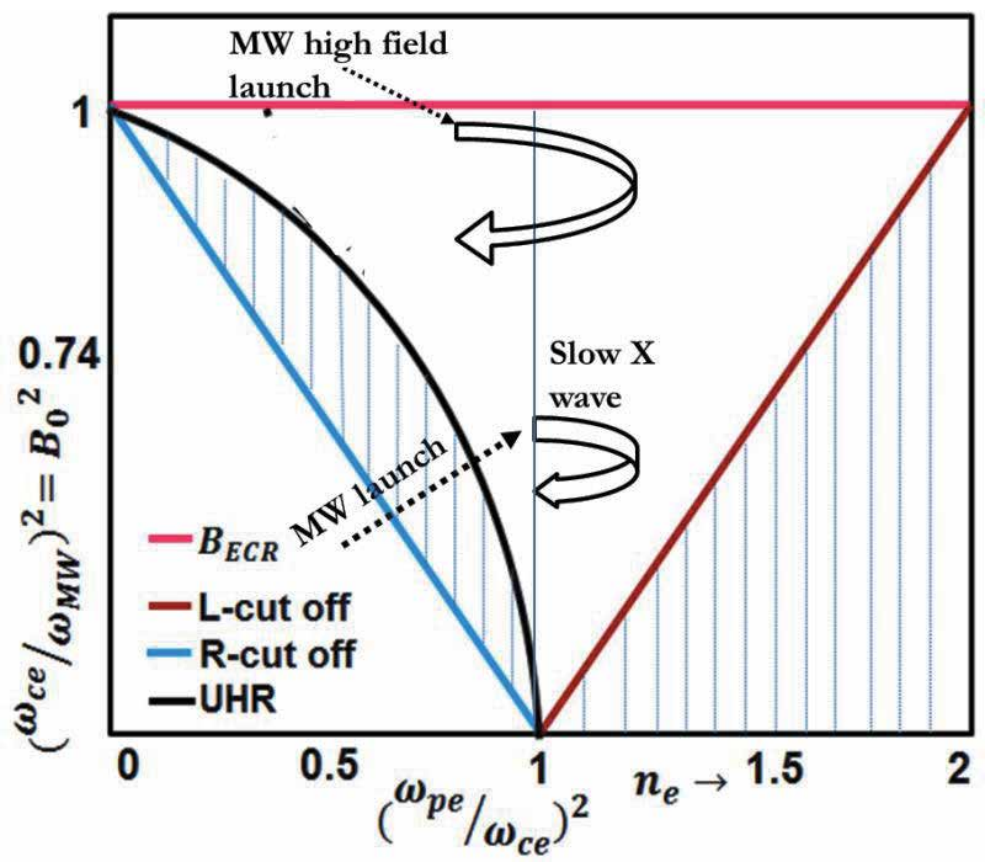

Figure 1.

The CMA diagram shows the propagation of microwave launched from high and low magnetic field side. The arrow bend implies the cut-off region and the mode conversion region near the upper hybrid resonance location. 
cyclotron resonance heating (ECR). It is to be noted that only in the ECR heating mechanism the energy is transferred directly from the microwave to the gaseous electrons. As the electrons gain sufficient energy by resonance, they ionize the neutral gases and thus form plasma. There are also other mechanisms for energy transfer from the microwave to the plasma. But the direct energy transfer is not possible in these cases. In fact, the energy is shared among the microwave and the plasma oscillation modes by means of collisional absorption or non-linear phenomenon. The plasma oscillation modes are excited by the ' $O$ ' and ' $X$ ' modes of microwaves. A detailed description of the energy damping mechanisms is discussed below.

\subsection{Electron cyclotron resonance (ECR)}

As discussed above, the ECR heating is an attractive tool in direct energy transferring to the plasma specifically in the ion source applications. The working principle of ECR mechanism is based on the frequency matching condition in which the microwave frequency with same polarization matches with electron cyclotron motion. It looks pretty much simpler in a qualitative approximation. But in case of quantification, it appears to be a non-deterministic method. It means along with the frequency, the phase difference between the microwave and electron motion also plays important role in energy transferring through ECR mechanism. Under frequency matching condition, if the phase difference between the microwave and the electron motion is in the same phase, then the electrons are accelerated by the microwave electric field. On the other hand, if it is $180^{\circ}$ out of phase, the electrons are decelerated. Practically, the temporal phase difference between the microwave and the electron motion is a random phenomenon. So, it becomes necessary to take an average energy gain temporally of the electrons for several microwave periods. It is demonstrated by several groups worldwide that the average temporal energy gain of the electrons has positive value if the averaging calculation is performed irrespective of the phase difference between the microwave and the electron cyclotron motion. It has been proved that the net energy gain is related non-linearly to the microwave electric field $\left(\propto E^{2}\right)$. In ECR heating-based plasma, the collisions in plasma can play important role on the confinement of the plasma particles, thermalizing the plasma particles, and also on the ionization rate. The collision properties of plasma are determined by two physical parameters: mean free path $\left(\lambda_{\text {mean }}=\frac{1}{n \sigma}\right)$ and collision frequency $(?=n \overline{\sigma v})$. Here, $\overline{\sigma v}$ is the product between cross-section and the velocity of plasma particles that is determined from the velocity distribution function (Maxwellian type). The particle confinement time is inversely proportional to the collision frequency. A contradiction arises from these two relations. The plasma can have a temperature even in collision-less case and follow the Maxwellian distribution. In collision-less case, the particle does not collide with each other and so there is no thermalization. But in ECR case, the kinetic energy of electrons increases, but there would be low temperature of the plasma. It suggests that there must be other mechanisms that are involved for the solution of this contradiction.

In most of the ECR ion source plasmas, the plasma is collision-less. But in other ion sources operating at microwave discharge, where the temperature of the plasma is comparatively lower and the pressure is also higher, a complete collision-less approximation is not valid. The other heating mechanisms that are involved in the collisional absorption condition must be taken into account. Considering the collisional term in the equation of motion,

$$
m_{e} \frac{\partial \tilde{v}}{\partial t}=e(\tilde{E}+\tilde{v} \times \tilde{B})-m_{e} \omega_{c} \tilde{v}
$$


Here, $\omega_{c}$ denotes the collision frequency. Then the dispersion relation is modified as:

$$
N_{O, X}^{2}=1-\frac{2 x(1+i Z-x)}{2(1+i Z)(1-x)-y^{2} \sin ^{2} \theta \pm \sqrt{y^{4} \sin ^{4} \theta \pm 4 y^{2}(1+i Z-x)^{2} \cos ^{2} \theta}}
$$

Here, $Z=\frac{\omega_{c}}{\omega}$. It can be observed that there is collisional term present in the modified dispersion equation of the wave propagation. This collisional term limits the attainable energy during the ECR heating unlike the collision-less case, as discussed before.

\subsection{Wave propagation in warm plasma condition}

Although the cold plasma approximation is not valid for the wave dispersion in the region very close to the cut-off, the effect of warm plasma condition cannot be ignored. This is because the wavelength in the latter case is not negligible compared to the scale length of the plasma parameters [14]. At the resonance, where the refractive index is infinity, the wavelength becomes equivalent to the electron larmor radius. So, the finite larmor parameter effect is not negligible and has to be considered. The larmor radius can be written as $\kappa^{\prime}=0.5\left(k_{\perp}^{2} v_{t h}^{2}\right) / \omega_{c}^{2}$ where $k_{\perp}$ denotes the perpendicular component of the wave vector with respect to the magnetic field ( $Z$-direction). For the non-relativistic case assuming the Maxwellian distribution function, the distance of frequency from the $n$th cyclotron harmonic resonance in terms of the Doppler shift unit is written as $\xi_{n}=$ $\left(\omega+n \omega_{c}\right) /\left|k_{z}\right| v_{t h}^{2}$. Here $k_{z}$ represents the $z$-component of the wave vector. The dielectric tensor is expressed as:

$$
\overline{\overline{\epsilon_{r}}}=\overline{\bar{I}}+\frac{\omega_{p e}^{2}}{\omega^{2}} \sum_{n=-\infty}^{n=\infty}\left[\begin{array}{ccc}
\epsilon_{x x} & \epsilon_{x y} & \epsilon_{x z} \\
\epsilon_{y x} & \epsilon_{y y} & \epsilon_{y z} \\
\epsilon_{z x} & \epsilon_{z y} & \epsilon_{z z}
\end{array}\right]
$$

where $\epsilon_{x x}=\frac{n^{2}}{\hat{\Gamma}} \overline{I_{n}} Z_{n} \epsilon_{x y}=-\epsilon_{y x}=i n \overline{I_{n}^{\prime}} Z_{n} ; \epsilon_{x z}=\epsilon_{z x}=-n \sqrt{\frac{2}{\hat{I}}} \overline{I_{n}}\left(1+\xi_{n} Z_{n}\right) ; \epsilon_{y y}=$ $\left(\frac{n^{2}}{\hat{I}} \overline{I_{n}}-2 \overline{I^{\prime}}\right) Z_{n} ; \epsilon_{y z}=\epsilon_{z y}=-i \sqrt{2} \overline{I_{n}}\left(1+\xi_{n} Z_{n}\right) ; \epsilon_{z z}=2 \xi_{n} \overline{I_{n}}\left(1+\xi_{n} Z_{n}\right)$.

Here $Z_{n}=Z\left(\xi_{n}\right)$ and $\overline{I_{n}}=e^{-\hat{z}} I_{n}\left(z^{\prime}\right)$ where $I_{n}$ is the modified Bessel's function of $n$th order and $Z$ is a function that is written as [15] $Z\left(\xi_{j}\right)=\frac{1}{\sqrt{\pi}} \int_{-\infty}^{\infty} \frac{e^{-s^{2}}}{s-\xi_{j}}$.

This dielectric tensor coming from warm plasma approximation has few new features that affect the wave propagation unlike the cold plasma approximation case. It can be seen from Eq. (23) that the dielectric tensor is not only a function of $\omega_{p e}$ and $\omega$ but also a function of the plasma temperature and the $k$-vector components. So, the dispersion relation yields a new kind of solution that is called electrostatic wave.

\subsection{Electrostatic wave}

Usually, the electric field of an electrostatic wave does not change with time. This fact is known from the derivation of the electrostatic field from a scalar 
potential $(\tilde{E}=-\tilde{\nabla} V)$, which makes $\tilde{\nabla} \times \tilde{E}$ to be zero. However, the time-dependent electric field is sometimes denoted as electrostatic wave as it obeys $\tilde{E}=-\tilde{\nabla} V$ relation. So a concrete example for calling an electric field to be electrostatic is the $k$-vector direction of electrostatic wave with respect to its electric field. If the kvector is parallel to the electric field, it is called as an electrostatic wave. It means $\tilde{\nabla} \times \tilde{E}=\tilde{k} \times \tilde{E}$ that makes the $\frac{\partial \tilde{B}}{\partial t}$ component to be zero. Therefore, the electrostatic wave does not have any magnetic field component.

A commonly occurred electrostatic wave in a warm plasma condition is named as the Langmuir wave [16]. The Langmuir wave is the main constituent of unmagnetized plasma that appears together with the ion-acoustic wave (IAW). In case of magnetized plasma, electrostatic waves are also present. In this case, if the electrons are displaced by some force, an electric field builds up to restore the electrons back to their initial position to maintain the plasma quasi-neutrality condition. Due to the very low inertia, the electrons will show an overshoot and oscillate around an equilibrium position. The frequency of oscillations is equivalent to the electron plasma frequency of the plasma. The dispersion relation of the Langmuir wave is written as [16]:

$$
\omega_{L}^{2}=\omega_{p e}^{2}+\frac{3}{2} k^{2} v_{t h}^{2}
$$

As the electron plasma oscillates very fast compared to the massive ions present in plasma, the massive ion motion is considered to be fixed in the $\mathrm{GHz}$ frequency scale (Langmuir frequency range). Although the frequency of the massive ion motion is very low compared to the Langmuir wave, the massive ions part will take part in the oscillations due to the electric field build-up. This low-frequency oscillations fall usually in the range of ion-acoustic wave frequency. The ion wave dispersion is obtained from the fluid equation as,

$$
\frac{\omega_{\text {IAW }}}{k_{\text {ion }}}=\left\{k_{B}\left(T_{e}+\gamma_{i} T_{i}\right) / M_{i}\right\}^{1 / 2}
$$

Usually, the plasma oscillations in the ion-acoustic frequency range lie in between few $\mathrm{kHz}$ to tens of $\mathrm{MHz}$.

There exists another kind of electrostatic wave in magnetized plasma, which is known as electron Bernstein waves (EBW). EBW exist in warm plasma conditions when the electron temperature has finite value. It is known that the superposition of the static magnetic field with the oscillating electric field of the plasma waves can make the electron orbit to be elliptical $[17,18]$. Now, if the magnetic field is increased further, the electron orbit will become a circular one as the Lorentz force dominates the electrostatic component $[17,18]$. The presence of EBW makes the electron gyrophase to organize in such a manner that the space charge distribution in plasma obtains a minima and maxima in the direction perpendicular to the externally applied magnetic field. It was shown [19] that the space charge accumulation is periodic. The charge accumulation propagates with a wavelength that is four times the electron larmor radius [19]. As the wavelength of the EBW is much lower than the length of a typical Langmuir probe tip, used for the plasma characterization, the Langmuir probe is unable to detect the EBW wave directly [20]. The dispersion of electron Bernstein wave (EBW) can be written as:

$$
\frac{k_{\perp}^{2}}{2 \omega_{p e}^{2} / v_{t h}^{2}}=2 \omega_{c}^{2} \sum_{n=1}^{\infty} n^{2} \frac{e^{-b} I_{n}(b)}{\omega_{M W}^{2}-n^{2} \omega_{c}^{2}}
$$


Here $k_{\perp}, v_{t h}$, and $I_{n}$ represent $k$-vector perpendicular component, electron thermal velocity and the Bessel's function, respectively. From Eq. (26), it can be seen that the $k$-vector becomes infinite at the cyclotron harmonic, i.e., $\omega_{M W}^{2}=n^{2} \omega_{c}^{2}$. Therefore, the EBW waves can have resonance at the harmonics of the cyclotron resonance frequencies. In microwave ion source, the resonance absorption of the EBW is possible at the harmonics of the cyclotron frequencies of the ECR magnetic field $\left(B_{E C R}\right)$ value.

\subsection{Mode conversion theory}

In microwave-generated magnetized plasma, the presence of plasma density gradient and the variation of the magnetic field make the wave propagation and its energy absorption unpredictable. It is difficult to estimate the wave trajectory from the simple linear uniform plasma theory [21]. It is natural that the wave would cross the boundaries shown in the CMA diagram by travelling up or down depending on the magnetic field variation and plasma density distribution. Inhomogeneous and anisotropic plasma can exhibit a wide variety of possibilities for the cut-off, resonance, cut-off-resonance and/or the back-to-back cut-off pairs. In inhomogeneous plasma, two or more waves can coexist that propagates in the plasma having density gradient. Although their polarization and propagation vector are different from one another, they can exhibit identical characteristics at some particular plasma regions having particular plasma loading conditions. At those particular scenarios, the waves can remain no longer distinguishable and therefore can convert into another. The mode conversion theory deals with establishing resonance characteristic in inhomogeneous plasma considering two different waves present in the plasma by taking into account the wave reflection, cut-off, resonance and absorption conditions. As the microwaves that are present in the microwave ion source plasma is dominated by the ordinary- and extraordinary-type microwave, the mode conversion theory is mainly focused upon considering the cut-off-resonance pair condition in plasma. The $X$ mode microwave is unable to propagate the dense plasma because it is reflected at R cut-off. On the other hand, O-mode microwave is able to propagate in the dense plasma, where it converts into the $\mathrm{X}$ mode microwave under certain condition that is obtained from Eq. (18). As per CMA diagram, the $X$ mode can have resonance at the UHR region. Therefore, before entering the UHR region, the launched microwave can convert into the $X$ mode based on the following mechanisms:

- If the $X$ mode microwave is launched from the high magnetic field side, the $X$ mode will not see the R cut-off (see Figure 1). It will propagate toward the UHR zone where it can convert into an EBW and an ion wave as per the literature. This method is known as $X-B$ conversion process.

- Another method in generating the EBW and ion wave is the $O-X-B$ mode conversion process. In this process, the $O$ mode microwave launched from the vacuum side crosses the $\mathrm{R}$ cut-off and converts into a slow $X$ mode after tunneling through the evanescent layer. The generated slow $X$ mode is then converted into an EBW and an ion wave near the UHR layer.

In microwave ion source plasma under mirror magnetic field configuration, there can coexist two types of components ( $O$ and $X$ modes) of the launched microwave. The ion source cavity acts as a resonator having comparable cavity dimension with respect to the launch microwave wavelength. Therefore, different 
types of propagating modes can coexist in the presence of plasma. This makes it difficult to identify which mechanism is actually allowing the $X$ mode to reach the UHR region. However, it is possible to estimate this mode conversion efficiency. For the case of $X$ mode microwave, the refractive index is written as:

$$
\frac{c^{2} k^{2}}{\omega^{2}}=1-\frac{\omega_{p e}^{2}}{\omega^{2}} \frac{\omega^{2}-\omega_{p e}^{2}}{\omega^{2}-\omega_{U H R}^{2}}
$$

Let us suppose the $X$ mode wave sees cut-off at the electron plasma frequency, $\omega_{p e 1}$. The wave cut-off condition yields

$$
\frac{\omega_{p e 1}^{2}}{\omega^{2}}=1-\frac{\omega_{c}}{\omega}
$$

After reflection at the cut-off, the wave propagates in the inhomogeneous and anisotropic plasma in the location where it will find a resonance (refractive index = $\infty)$. The resonance is called upper hybrid resonance where electron plasma frequency is $\omega_{p e 2}$. The corresponding Eq. (27) becomes then

$$
\frac{\omega_{p e 1}^{2}}{\omega^{2}}=1-\frac{\omega_{c}^{2}}{\omega^{2}}
$$

By dividing Eq. (28) by Eq. (29), one gets

$$
\frac{\omega_{p e 2}^{2}}{\omega_{p e 1}^{2}}=1+\frac{\omega_{c}}{\omega}=\frac{n_{e 2}}{n_{e 1}} \approx 1+\frac{\Delta x}{L}
$$

where $\Delta x$ and $L$ denote the distance between the cut-off and the upper hybrid resonance layer and the length parameter, respectively. From Eq. (30), one obtains

$$
\Delta x=\frac{\omega_{c}}{\omega} L
$$

Now, for the wavelength $\lambda \ll L$, the $\Delta x$ will be many times the wavelength. So the wave will not be able to reach the upper hybrid resonance point. The mode conversion efficiency is determined by the penetration depth of the wave into the plasma. The effective mode conversion can be achieved when the parameter $L$ is comparable to the wavelength, $\lambda$. In another way, from Eq. (31), it is also seen that mode conversion can be improved if the magnetic field is reduced.

If a strong electromagnetic field is present in the ion source cavity, the plasma particle follows the relation $v_{E} \leq v_{t h e} ; v_{E}=e E / m \omega$, where $v_{E}, E, e, m$ and $v_{\text {the }}$ are wave phase velocity, electric field intensity, electron charge, electron mass and electron thermal velocity, respectively. This condition leads to plasma parameters to vary with time. In effect, the non-linear effect such as parametric instability comes into play. Off-course, the parametric decay happens above certain threshold value of the electric field and the microwave energy is shared among the plasma waves and the microwave through the non-linear interaction phenomenon.

\subsection{Parametric decay}

As the plasma parameters vary with time under the conditions of intense electric field of the microwave, the corresponding velocity becomes close to the electron thermal velocity. It is known that the $X$ mode electric field accumulates near the 
UHR region with time due to its propagation from the cut-off region to the UHR region. The accumulated electric field will be able to share some part of its energy through non-linear interactions to the oscillating modes present in the plasma. This non-linear energy coupling between the $X$ mode microwave and the oscillation modes is known as parametric instability. It is demonstrated that the $X$ mode through this type of instability couples energy to the Bernstein wave and ion wave near the UHR region. The $X$ mode normally contains the longitudinal component of the electric field (TM type). Considering the $x$-component of the $X$ mode propagation from Eq. (17), the dispersion of $X$ mode wave can be written as follows:

$$
\left(\omega^{2}-\omega_{U H R}^{2}\right) E_{x}+i \frac{\omega_{p e}^{2} \omega_{c}}{\omega} E_{y}=0
$$

From Eq. (11), as the $X$ mode approaches the UHR, the term $\omega^{2}-\omega_{U H R}^{2} \rightarrow 0$ and so $E_{y}$ would be zero. Therefore, the component $E_{x}$ will remain non-zero at the UHR, which is directed in the direction of propagation of $X$ mode. This means the $X$ mode at the UHR becomes electrostatic. The $X$ mode microwave that becomes an electrostatic wave at the UHR matches with the Bernstein wave and the ion wave. With the two oscillation modes that are coupled with the $X$ mode electric field $\left(E_{0}\right)$, the motion of one of the modes (Bernstein wave) can be expressed in terms of the simple harmonic oscillator.

$$
\frac{d^{2} x_{1}}{d t^{2}}+\omega_{1}^{2} x_{1}=C_{1} x_{2} E_{0} \cos \omega_{0} t
$$

Here the motion of the first oscillator (amplitude $x_{1}$ and the resonant frequency $\left.\omega_{1}\right)$ is driven by the time-dependent electric field of the $X$ mode and the amplitude $\left(x_{2}\right)$ of the second oscillator. The equation of the motion for the second oscillator $x_{2}$ is:

$$
\frac{d^{2} x_{2}}{d t^{2}}+\omega_{2}^{2} x_{2}=C_{2} x_{1} E_{0} \cos \omega_{0} t
$$

Let us assume, $X_{1}=\overline{x_{1}} \cos \omega^{\prime} t$ and $X_{2}=\overline{x_{2}} \cos \omega^{\prime \prime} t$. In the absence of any nonlinear interaction, it can be expressed as $\omega^{\prime}=\omega_{1}, \omega^{\prime \prime}=\omega_{2}$ and $\omega_{0}=\omega_{1}$. But in the presence of non-linear interaction, this is incorrect. For the case of non-linear interaction, as the driving terms cause frequency shifting, the frequency $\omega_{2}$ is approximately equal to $\omega^{\prime \prime}$. However, in the absence of non-linear interaction, the solution from the system of coupled Eqs. (33) and (34) is obtained as:

$$
\omega^{\prime \prime}=\omega_{0} \pm \omega^{\prime}
$$

Now if $\omega^{\prime}$ is small such that both values of $\omega^{\prime \prime}$ lie within the bandwidth of oscillator, $x_{2}$, there exist two oscillators: $x_{2}\left(\omega_{0}+\omega^{\prime}\right)$ and $x_{2}\left(\omega_{0}-\omega^{\prime}\right)$. Under this assumption, solving the coupled Eqs. (33) and (34), the following frequency matching condition is arrived:

$$
\omega_{0} \cong \omega_{2} \pm \omega_{1}
$$

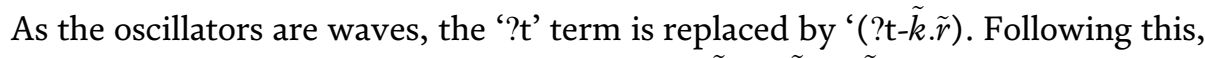
a new matching condition of k-vector is reached, $\tilde{k_{0}}=\tilde{k_{2}} \pm \tilde{k_{1}}$.

The frequency and k-vector matching conditions correspond to the energy conservation and momentum conservation following the quantum mechanics theory. It is proved that [15] the $X$ mode generates two waves through parametric decay: a 
high-frequency Bernstein wave and a low-frequency ion wave following the frequency matching condition. This leads to the generation of secondary peaks around the launched microwave frequency, which is called as sideband. Therefore, the simultaneous presence of the sideband peaks around the launched frequency signifies the parametric decay near the mode conversion region.

The parametric decay occurs above a certain threshold value, which actually depends on the damping rate of the oscillator. If the damping rates $\Gamma_{1}$ and $\Gamma_{2}$ for two oscillators $x_{1}$ and $x_{2}$, respectively, are introduced in Eqs. (33) and (34), one can conclude that the lowest threshold occurs at the exact frequency matching condition. The corresponding threshold electric field is obtained as [19]:

$$
C_{1} C_{2} E_{0 \text { threshold }}^{2}=4 \omega_{1} \omega_{2} \Gamma_{1} \Gamma_{2}
$$

\subsection{Damping of electrostatic and electromagnetic energy}

Electrostatic waves generated in the plasma through the parametric decay instability can damp their energy to the plasma particles and thus increase the plasma density. When the phase velocity of the electrostatic wave becomes comparable to the thermal velocity of the plasma particles, the energy is transferred from the wave to the plasma particles and is known as Landau damping mechanism. In microwave ion source plasma, density can be increased 2-3 times more than the ECR heating mechanisms through the off-resonance heating mechanism. For this reason, the offresonance condition is used to create favorable conditions of the upper hybrid resonance heating. Under certain plasma temperature, the electrostatic wave can transfer energy resonantly to the plasma particles if the wave phase velocity matches the plasma particle velocity. In some cases, the plasma particle velocity can be higher than the wave phase velocity. Under this condition, the plasma particle can transfer energy to the wave. The Landau damping mechanism follows the equation written below:

$$
I_{m}\left(\frac{\omega}{\omega_{p e}}\right)=-0.2 \sqrt{\pi}\left(\frac{\omega_{p e}}{k v_{t h}}\right)^{3} e^{-12 k^{2} \lambda_{D}^{2}}
$$

The exponential term on the right hand side represents that the Landau damping will be small for small value of $k \lambda_{D}$. The Landau damping phenomenon is applicable for both case of electrons and ions. The electrostatic wave whose frequency falls in the range of electrons can heat the electrons only in the parallel direction with respect to the magnetic field. In case of electrostatic ion wave, such restriction does not apply. This is because the electrons are strongly magnetized and so will not be able to move across the magnetic field.

In case of a compact microwave plasma device where the microwave wavelength becomes comparable to the device dimension, the cavity resonant mode can also play crucial role in damping the electromagnetic energy to the plasma particles. The presence of multiple cavity modes in the plasma can produce modulated wave due to the interaction between each pairs of the cavity resonant modes. The generated modulated wave propagates in the plasma and damps its energy to the plasma particles where the frequency of the modulated wave matches the local plasma frequency of the plasma particles.

\section{Simulation modeling of MW interaction in plasma}

For the MW interaction modeling during the plasma evolution, a schematic of the computational domain is shown in Figure 2. The computational domain consists 


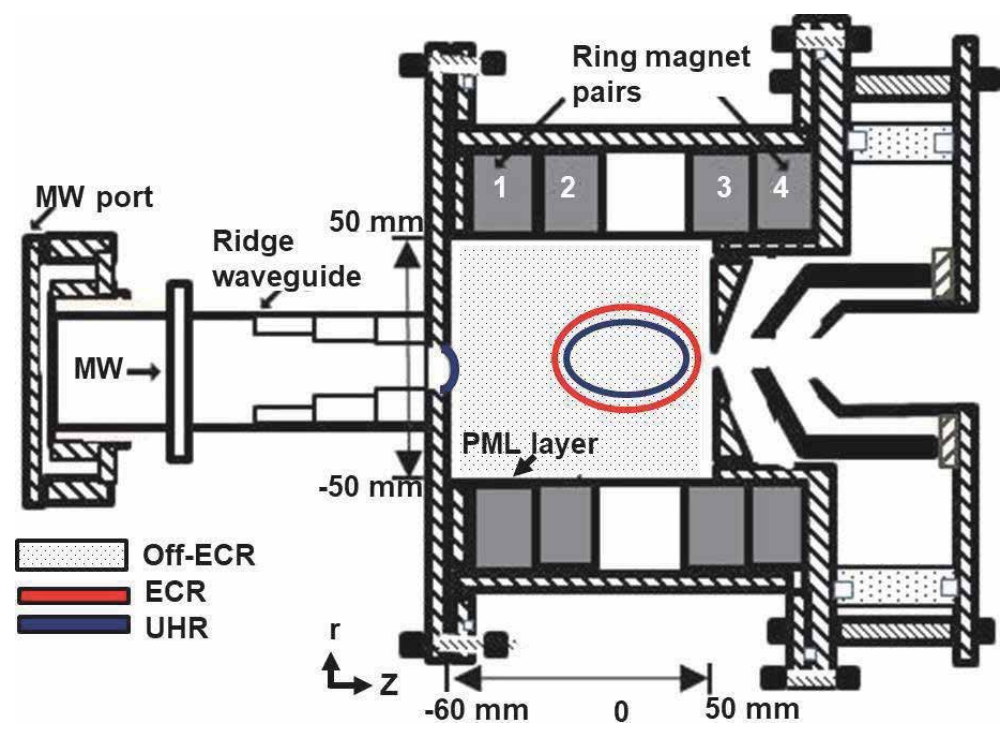

Figure 2.

Simulation domain of the MW ion source.

of a microwave coupled reactor, which is a cylindrical plasma cavity. A MW of frequency $2.45 \mathrm{GHz}$ is injected into the microwave coupled reactor through a ridge waveguide port (on the left side of Figure 2) to ionize the gaseous particles, thus forming the plasma that is confined under the mirror magnetic field configuration. The reactor has dimension of $107-\mathrm{mm}$ and $88-\mathrm{mm}$ diameter. The microwave power is fed into a cavity resonator through a tapered waveguide. The waveguide is tapered by embedding four ridge sections having different ridge length, ridge gap and ridge width on the inner sidewalls of the waveguide. The ridge dimensions are optimized from the analytical calculation as well as from the electromagnetic simulation. The mirror magnetic field is created by using two pairs of ring magnets that surround the microwave coupled reactor [22]. On the right side of the microwave coupled reactor (Figure 2), the ion beam extraction system is attached through a 5 -mm hole on the wall of the reactor. The similar computational domain is used in the experimental set-up (Section 5) to validate the simulated data. Here, the finite element method (FEM)-based COMSOL model is used [22].

The MW propagation and the plasma evolution are assumed to be decoupled to each other during the simulation modeling in the temporal scale [10]. The MW electric fields $(\mathrm{E})$ are averaged for some MW periods before putting their value in the plasma model and the resultant field is given as input to the plasma model. The electron's momentum equation is time integrated along with Maxwell's equations for some MW periods until the MW model of the FEM gets a periodic solution of the equation to transfer an average power to the particles over such a period. The FEM model continues this process until it gets a steady state solution for at least $\sim 10^{3}\left(\omega / \nu_{\mathrm{m}} \sim 10^{3}\right) \mathrm{MW}$ periods. Here, $\omega$ is MW frequency and $\nu_{\mathrm{m}}$ is electron's momentum transfer frequency. Since the electrons stay in the ECR zone for a very short time (transit time) duration, it causes non-local kinetic effects. This results in the de-phasing between the velocity and field oscillations that becomes very difficult to describe using the fluid model. This problem is resolved by introducing the effective collision frequency $\left(\nu_{\text {eff }}\right)$ in the simulation to converge the solution. In the low pressure condition, for the collision-less heating, $\nu_{\text {eff }}$ has to be in the order of inverse transit time for the electrons [10]. 
In case of MW model, the equations for the electric field are solved in frequency domain while keeping the other parameters in time domain. In the beginning, the FEM model started from the Maxwell's equations in order to justify the modeling approach. The MW electric fields are changing with time at a frequency of $\omega / 2 \pi$. During the plasma evolution, the total electric field (E) value in the $\mu$ s time zone is regarded as a resultant quantity (or total) that comes from the superposition between the MW and ambipolar-type electric field of the plasma. The modification of the resultant electric field follows the equation that is given below:

$$
\nabla \times \mu^{-1}(\nabla \times E)-k_{0}^{2}\left(\varepsilon_{r}-\frac{i \sigma}{\omega \varepsilon_{0}}\right) E=0
$$

The notations used in the above equations $k_{0}, \varepsilon_{r}, \sigma$ and $E$ are the vacuum wavenumber, relative permittivity, the total electric field and the plasma conductivity in full tensor form, respectively. This plasma conductivity is a function of plasma density, collision frequency and B-field. So, all types of total electric field components are possible to estimate as the FEM model can determine the above parameters for some particular plasma loading conditions during its evolution. The model also considers that the $E$-field evolution in the sub-nanosecond region is well separated from the total E-field quantity. This means the total E-field changing during the $\mu$ s region is composed of the MW electric field and the ambipolar electric field.

For representing the MW propagation in an infinite space, the perfectly matched layers (PML) are introduced in the computational domain as shown in Figure 1. The present FEM model considers the electron transport properties to follow the Boltzmann distribution function. The distribution is an integro-differential equation in phase space $(r, u)$ that cannot be solved efficiently. For this reason, the FEM model assumes the plasma as a fluid following the drift diffusion approximation. The assumption is adapted from the Boltzmann equation that is multiplied by some weighing function and then integrated the resulted function over the velocity space. This exercise yields completely three-dimensional and time dependent equations $[10,23]$. During the modeling, FEM assumes that ion motion is negligible with respect to the electron motion in the timescale (ns) of MW. Additionally, the electron density is constant spatially within the ECR surface. The Debye length is also assumed to be much smaller than the interaction length of the MW. The average value of the electron velocity on the microwave timescale is obtained from the assumption of Maxwellian distribution function and from the first derivative of the Boltzmann equation. The following drift-diffusion equations are shown that are used to compute the electron density and electron energy density:t

$$
\begin{gathered}
\frac{\partial n_{e}}{\partial t}+\nabla \cdot\left[-n_{e}\left(\mu_{e} \cdot E\right)-D_{e} . \nabla n_{e}\right]=R_{e} \\
\frac{\partial n_{\varepsilon}}{\partial t}+\nabla \cdot\left[-n_{\varepsilon}\left(\mu_{\varepsilon} \cdot E\right)-D_{\varepsilon} \cdot \nabla n_{\varepsilon}\right]+E . \Gamma_{e}=R_{\varepsilon}
\end{gathered}
$$

The term in Eq. (42) is $E . \Gamma_{e}=e n_{e} v_{e} \cdot E_{\text {ambipolar }}-\Pi$. This is a heating term $E . \Gamma_{e}$ that comprises two components. The first component means the electrons are getting energy through the ambipolar field during the plasma evolution. The second component $(\Pi)$ signifies the MW absorption power $\left(n_{e}\left\langle v_{e} \cdot \tilde{E}\right\rangle\right)$ that is carried by the electrons in the plasma. The other terms $n_{e}, v_{e}, R_{e}, \Gamma_{e}, \mu_{e}, \mu_{\varepsilon}, E, D_{e}, n_{\varepsilon}, R_{\varepsilon}, \Gamma_{\varepsilon}, n_{\varepsilon}$ and $D_{\varepsilon}$ are the electron density, average electron velocity, the source term of the electrons, electron flux, the mobility of the electrons $\left(4 \times 10^{4} \mathrm{~m}^{2} /(V s)\right)$, electron 
energy mobility, total electric field, electron diffusivity, electron energy density, energy loss/gain from inelastic collisions, electron energy flux, electron energy mobility and electron energy diffusivity, respectively. Some of the terms described above, the electron's diffusivity, mobility and energy diffusivity, are estimated following the relations that are given below:

$$
D_{e}=\mu_{e} T_{e} ; \mu_{\varepsilon}=(5 / 3) \mu_{e} \text { and } D_{\varepsilon}=\mu_{\varepsilon} T_{e}
$$

The above written electron transport properties represent full tensor parameters in which the tensor term electron mobility is influenced by the magnetic field. The electron mobility without the magnetic field $\left(\equiv 1 \times 10^{25} / N_{n}\right)$ and with the magnetic field is the known parameters. Here, $N_{n}$ represents the neutral density of the gaseous particles. The electron source term in Eq. (40) is obtained from the relevant plasma chemistry that is expressed below with the help of the rate coefficients $R_{e}=$ $\sum_{j=1}^{M} x_{j} k_{j} N_{n} n_{e}$. The presented symbols $M, x_{j}$ and $k_{j}$ represent the number of reactions, the mole fraction of a specific species and the rate co-efficient for the reaction $\mathrm{j}$, respectively [10]. The energy loss terms as shown in Eq. (41) is written for all the reactions as $R_{\varepsilon}=\sum_{j=1}^{M} x_{j} k_{j} N_{n} n_{e} \Delta \varepsilon_{j}$. The notation $\Delta \varepsilon_{j}$ denotes the energy loss that occurs from the reaction $\mathrm{j}$. The energy source and the loss terms are calculated inherently in the FEM model. The rate coefficients mentioned in the above expressions are taken from the cross-section data as per the relation $\mathrm{k}_{\mathrm{k}}=$ $\gamma \int_{0}^{\infty} \varepsilon \sigma_{\mathrm{k}}(\varepsilon) \mathrm{f}(\varepsilon) \mathrm{d} \varepsilon$, where $\gamma$ is $\left(2 q / m_{e}\right)^{1 / 2}$. In this expression, the symbols $\mathrm{m}_{\mathrm{e}}, \varepsilon, \sigma_{\mathrm{k}}$ and $f$ are represented as the mass of electron, energy of electron, cross-section for the reaction and the electron energy distribution function (Maxwellian) for the electrons, respectively.

The description for the boundary conditions taken during the plasma simulation is as follows. The plasma chamber wall is kept at ground potential. The reflections, secondary emission and also the thermal emission from the electrons are assumed to be negligible at the wall boundaries. In effect of that the electron flux and electron energy flux at wall boundary can be written as $\mathrm{n} \cdot \Gamma_{\mathrm{e}}=\left(\frac{1}{2} \mathrm{v}_{\mathrm{e}, \mathrm{th}} \mathrm{n}_{\mathrm{e}}\right)$ and $\mathrm{n} \cdot \Gamma_{\varepsilon}=\left(\frac{5}{6} \mathrm{v}_{\mathrm{e}, \mathrm{th}} \mathrm{n}_{\mathrm{e}}\right)$, respectively. In addition, the heavy plasma particle losses at the boundary walls and their migration are considered to be originating from only the surface reactions and ambipolar electric field, respectively. A fixed power is maintained that is absorbed by the plasma $\left(\mathrm{P}_{\text {absorbed }}\right)$. This is done by re-adjusting the normalization factor $(\alpha)$ during the moment of the plasma evolution (ns to $\mu \mathrm{s}$ ). The normalization of the plasma absorbed power follows the relation $\mathrm{P}_{\text {absorbed }}=\alpha \iiint \mathrm{n}_{\mathrm{e}} \mathrm{P}_{\text {set }} \mathrm{dV}$. Here, $\mathrm{P}_{\text {set }} \equiv$ $-\mathrm{e}\left\langle\tilde{\mathrm{v}}_{\mathrm{e}} . \tilde{\mathrm{E}}\right\rangle_{t}$ is represented as the average set power applied to the cylindrical plasma cavity. This normalization helps in convergence of the solution and avoids any disproportionate absorption of the power by the plasma. Here, a fixed plasma absorbed power of $70 \mathrm{~W}$ is chosen to benchmark its results with the experimental findings that are reported taking the boundary conditions $[23,24]$ similar to the computation system and the operating conditions.

In the MW-plasma simulation model, the instant of MW launch is taken as reference ( $t=0 \mathrm{~s}$ ) when the MW is launched into the cavity. The MW is launched in right $(\mathrm{R})$ hand mode ( $\mathrm{R}$ mode is extraordinary type) using the four step ridge waveguide. This makes the $\tilde{E}$-field intensity to be maximum in the center of the cavity that is propagating in parallel to the externally applied magnetic field $[25,26]$. As soon as the MW is launched, it continues to interact with the gas particles on their propagation timescales (ns). To understand and visualize the profile modification of the electrical field from the start of MW launch to the steady state plasma generation and also their impact on the power coupling to the plasma 
with time, total breakdown time is split into some discrete values such as $10 \mathrm{~ns}$, $\sim 67 \mathrm{~ns}, \sim 158 \mathrm{~ns}, 452 \mathrm{~ns}, 630 \mathrm{~ns}, \sim 2 \mu \mathrm{s}, 2.5 \mu \mathrm{s}, 3 \mu \mathrm{s}, \sim 5 \mu \mathrm{s}, \sim 8 \mu \mathrm{s}, \sim 20 \mu \mathrm{s}, \sim 40 \mu \mathrm{s}$, $250 \mu \mathrm{s}$ and $300 \mu \mathrm{s}$. The simulation is exercised with an argon gas. The initial conditions for the calculation of the plasma parameter evolution are plasma density $\left(1 \times 10^{12} \mathrm{~m}^{-3}\right)$ and plasma temperature $(\sim 4 \mathrm{eV})$. These parameters are used initially to estimate the plasma conductivity $\sigma$. Then the electric field is estimated by putting the above initial conditions in Eq. (39). The estimated field values are later utilized to find out the temporal variation of different plasma parameters and so electric field components' self-consistency.

The present FEM model uses different solvers sequentially to compute the magnetic field distribution first throughout the computational domain. Then the solvers related to the frequency-transient analysis are used to calculate the MWplasma parameters. A complete computational flowchart for the magnetic field as well as the MW-plasma is depicted in Figure 3. The typical number of degrees of freedom that is used for solving the magnetic field is approximately 54,525. The FEM magnetostatic model uses the equation-based mesh adaptation technique to generate the extremely fine mesh size on the ECR surfaces. The mesh element has

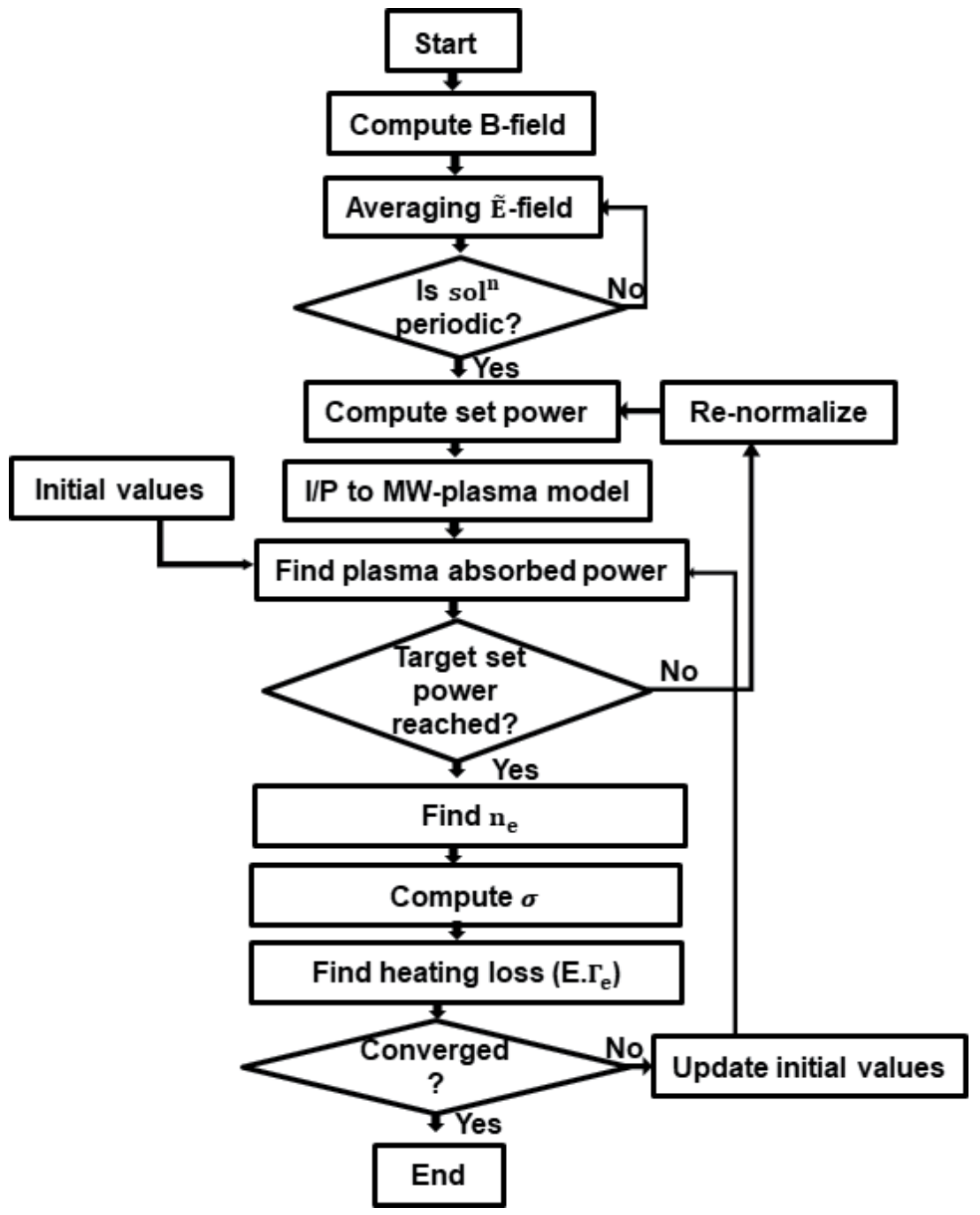

Figure 3.

Simulation flowchart of MW plasma interaction in COMSOL Multiphysics. 
minimum and maximum size for the magnetic field and microwave-plasma calculations are $0.2 \mathrm{~mm}$ and $2.4 \mathrm{~mm}$, respectively. The effect of the different edges that are involved in the computational domain is taken into account by keeping the maximum and minimum mesh element size at $0.5 \mathrm{~mm}$ and $0.0055 \mathrm{~mm}$, respectively. The magnetic field estimated is used in the MW-plasma model to estimate the tensor plasma parameters. For the case of MW-plasma simulation, the number of degrees of freedom used for the solution is approximately 47,005.

Due to the dephasing as discussed above, the current FEM model uses the concept of effective collision frequency $\left(\nu_{\text {eff }}\right)$ to describe the sudden phase decoherence. In the dephasing situation, the phase relationship between velocity and the electric field oscillation is destroyed in the temporal scale due to which the electrons experience a large field variation in the ECR surface. The accelerating electric field transfers energy to the electrons that are residing on the ECR surface only for a small time duration in the time range of resonant cyclotron motion of the electrons. The electrons also experience spatial density variations while oscillating across the ECR surface. A large density variation across the ECR surface also generates the radial ambipolar electric field on the resonance surface and so there is a possibility of de-phasing that can happen at the resonance zone.

As the phase de-coherence may happen between electron gyro motion and the MW oscillatory E-field, the electrons get accelerated and deccelerated assymetrically. This temporal asymmetry of acceleration and decceleration in opposite cycle are responsible for transferring an effective energy to the electrons. The effective collision frequency $\nu_{\text {eff }}$ is estimated using the relation $\nu_{\text {eff }}=\sqrt{v_{e} \omega / \delta_{B}}$, where $\mathrm{v}_{\mathrm{e}}$ is the electron thermal speed and $\delta_{\mathrm{B}}$ is the gradient of magnetic field. The value of $\nu_{\text {eff }}$ is few orders more than that of actual collisional frequency that an electron encounters with the gaseous particles. However, it is to be noted that the effect of $\nu_{\text {eff }}$ is insignificant on the power absorption profile. Instead it helps in overcoming the numerical instability. For obtaining a steady-state solution, the number of MW periods has to be in the order of $\omega / \nu_{\text {eff. }}$. This makes computation less rigorous than the case of actual collisions, if considered.

The evolution of the radial $\left(E_{\mathrm{r}}\right)$ and axial $\left(E_{\mathrm{z}}\right)$ components of the electric fields during the plasma evolution plays an important role in the MW coupling to the plasma and so the plasma parameters as discussed above. To support these facts, the

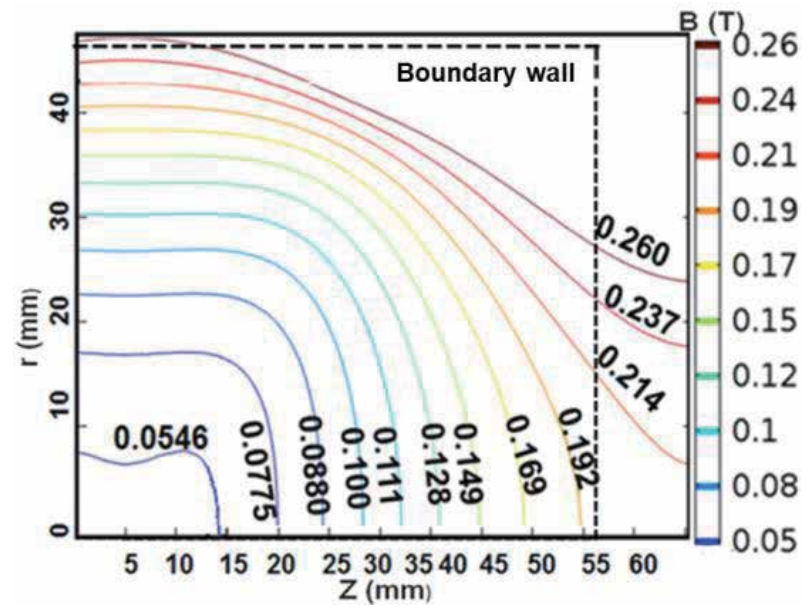

Figure 4.

Magnetic (B) field contour inside the quadrant section of the plasma chamber, which has cylindrical axis symmetry. The B-field is also simulated using COMSOL software. The narrow contour area near $0.088 \mathrm{~T}$ line is the ECR zone (corresponding to $0.0875 \mathrm{~T}$ ). The span of ECR zone is around $z= \pm 24 \mathrm{~mm}$ and $r= \pm 28 \mathrm{~mm}$. 
evolution of the different components of the electric fields and correspondingly the plasma parameters is shown in the results and discussion section given below to study their effects on the plasma parameters. To understand the resonance zone in the plasma chamber, the required magnetic field contours are shown in Figure 4.

\section{Results and discussion}

This section of the chapter shows the temporal behavior of the electric fields, power deposition and the corresponding variation of plasma density and hot electron temperature from the start of MW launch to the steady state condition.

\subsection{Behaviors of total electric field $(|E|)$ during plasma evolution}

Figure 5(a) and (b) shows the evolution of the radial profiles of the total electric field taken on the different axial locations for two time instances, $3 \mu \mathrm{s}$ and $20 \mu \mathrm{s}$, after the MW launch. The radial profiles show the total electric field intensity to be more near the MW launching port $(\mathrm{z}=-40 \mathrm{~mm})$. As one moves toward the extraction $(z=-60 \mathrm{~mm})$, its values is decreased because of the plasma shielding effect.

A sharp change in the E-field is shown in Figure 5(a) at the time, $t=\sim 3 \mu$ s near the $2.45 \mathrm{GHz}$ ECR surface ( $\mathrm{r} \approx 23 \mathrm{~mm}$ ) for the planes, $\mathrm{z}= \pm 20 \mathrm{~mm}$. The strong inhomogeneity in the E-field implies the absorption of the MW power at the same locations [27]. The power absorption location is also dependent on the magnetic field profile as well within the cylindrical cavity. Another figure, Figure 5(b) shows the radial E-field pattern across the different planes for time, $t=20 \mu \mathrm{s}$. The inhomogeneous part of the E-field profile looks similar to Figure 5(a) for the case of $\mathrm{z}=-20 \mathrm{~mm}$ plane. But the intensity of the E-field is being reduced with time due to the plasma shielding. One can observe that the inhomogeneous part of the E-field is shifted toward the off-ECR regime $(\mathrm{r} \approx 28 \mathrm{~mm})$ from the ECR surface with the increase of time. Therefore, the power absorption region is being shifted from the ECR zone to the off-ECR zone. This effect is visible in the power deposition location throughout the cylindrical cavity in Figure 6. Figure 5(a-d) shows the corresponding shifting of the power deposition location from the ECR zone to the off-ECR zone or UHR zone with time. As the plasma reaches steady state during the time, $\mathrm{t}=20 \mu \mathrm{s}$, the evolution of the plasma density and temperature is shown in Figure 7.
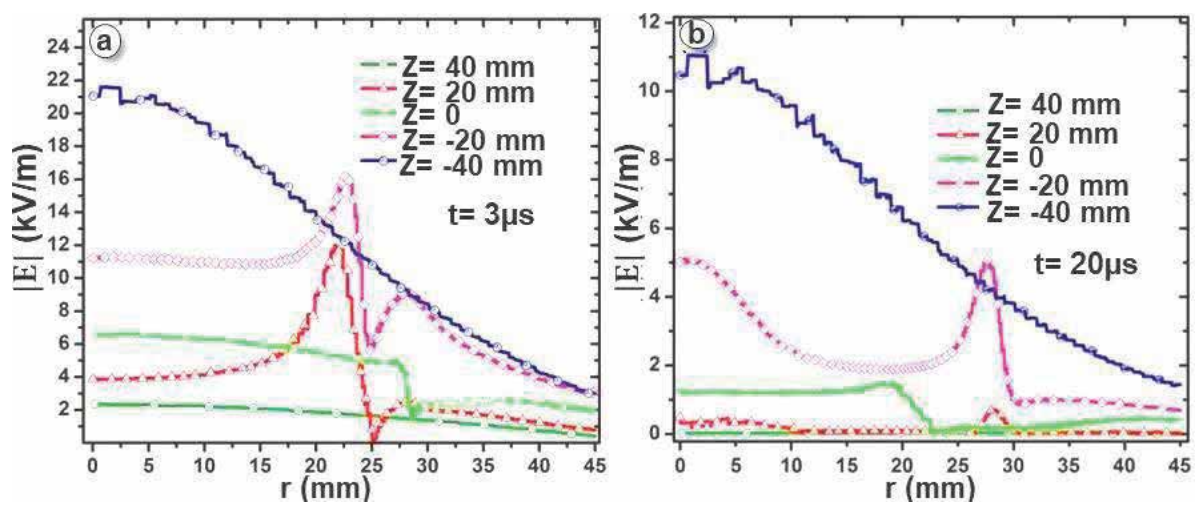

Figure 5.

(a) Total electric field ( $|\mathrm{E}|)$ magnitude during gas ignition time of $\sim 3 \mu s$, (b) total electric field $(|\mathrm{E}|)$

magnitude during gas ignition time of $\sim 20 \mu$ s. 


\subsection{Time evolution of plasma with power deposition}

Figure 6a shows that the MW power is being deposited exactly on the ECR $(\sim 0.0875 \mathrm{~T})$ surface corresponding to the launch MW frequency of $2.45 \mathrm{GHz}$ when the plasma density is low. But as time passes (see Figure $\mathbf{6 b - d}$ ), the power deposition location gets shifted in the off-ECR or upper hybrid resonance (UHR) regime. The UHR zone is a region where the two conditions $n_{e}<n_{\text {crit }}$ and $\mathrm{B}<\mathrm{B} \mathrm{ECR}$ are satisfied [26-28]. The term $n_{\text {crit }}$ represents the critical density for the MW frequency, $2.45 \mathrm{GHz}$ that is $7.4 \times 10^{16} \mathrm{~m}^{-3}$. If one can compare Figures $6(\mathrm{c})$ and $7(\mathrm{a})$, one can visualize that the plasma density $\left(\sim 1.3 \times 10^{17} \mathrm{~m}^{-3}\right)$ crosses the critical density from $\sim 2.5 \mu$ s onwards and the plasma density that is above the critical density is denoted as overdense plasma. So as the overdense plasma is achieved, the electrons get accelerated by less amount of MW energy on the ECR surface. Correspondingly, the plasma bulk temperature increases from the start of MW launch $(\mathrm{t}=0 \mathrm{~s})$ to the instant of $\sim 630 \mathrm{~ns}$. It is evident from Figure 7a that the plasma bulk electron temperature increases and becomes steady near a value of $\sim 80 \mathrm{eV}$. Then the plasma bulk temperature decreases in a faster way with further increase of time. Hence, one can conclude that heating through ECR process is being ceased to occur with the further increase in time. The causes to increase the absorbed power density is expected to be highest $\left(\sim 5 \times 10^{7} \mathrm{~W} / \mathrm{m}^{3}\right)$ on the ECR surface during the time, $\mathrm{t}=630 \mathrm{~ns}$.

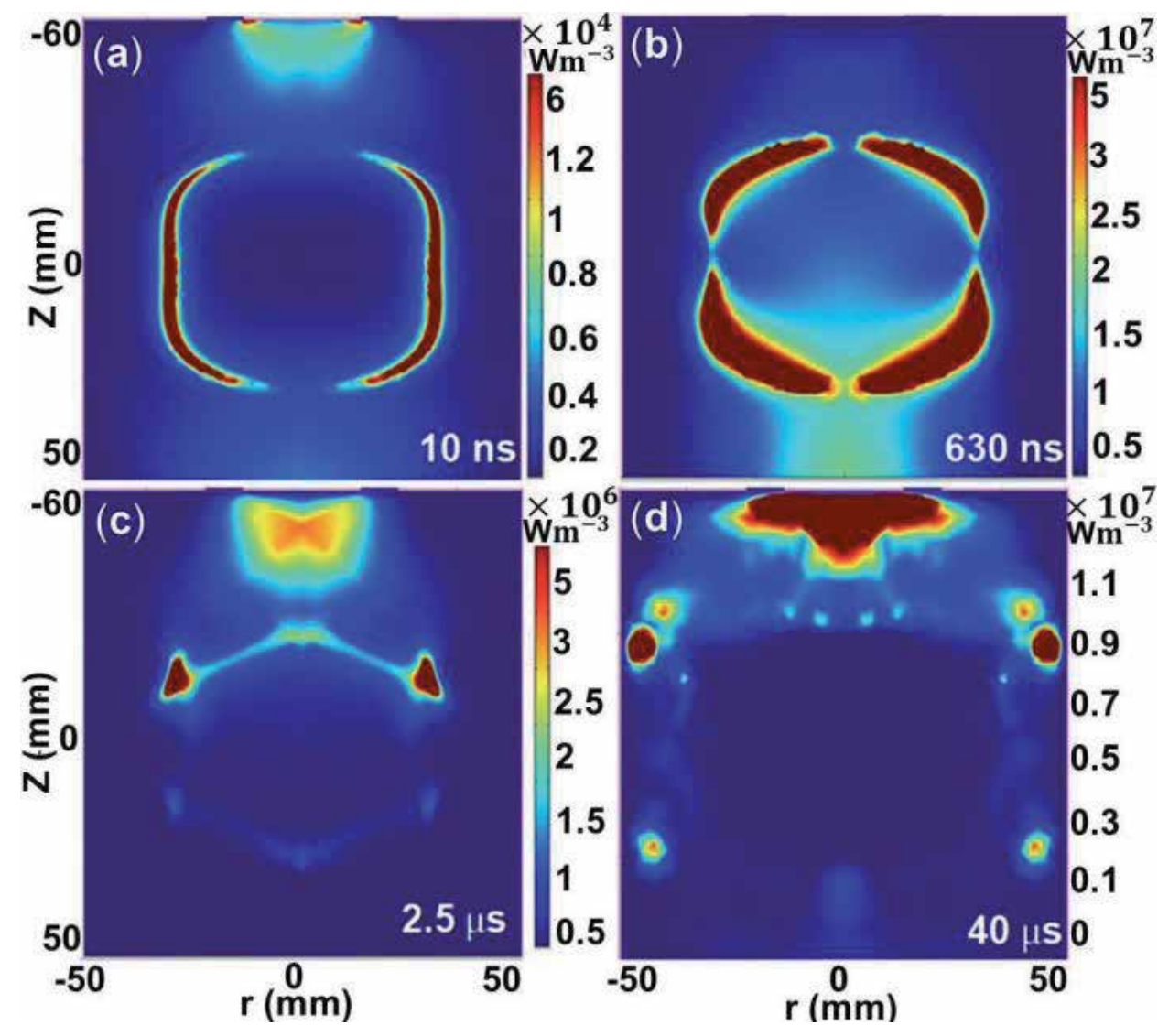

Figure 6.

Power deposition density at different time steps for $70 \mathrm{~W}$ of absorbed power. (a) $t=10 \mathrm{~ns}$, peak power density is $6.6 \times 10^{4} \mathrm{~W} / \mathrm{m}^{3}$, (b) $t=630 \mathrm{~ns}$, peak power density is $1.43 \times 10^{7} \mathrm{~W} / \mathrm{m}^{3},(c) t=2.5 \mu \mathrm{s}$, peak power density is $5.12 \times 10^{6} \mathrm{~W} / \mathrm{m}^{3}$ and $(d) t=\sim 40 \mu \mathrm{s}$, peak power density is $1.67 \times 10^{7} \mathrm{~W} / \mathrm{m}^{3}$. 

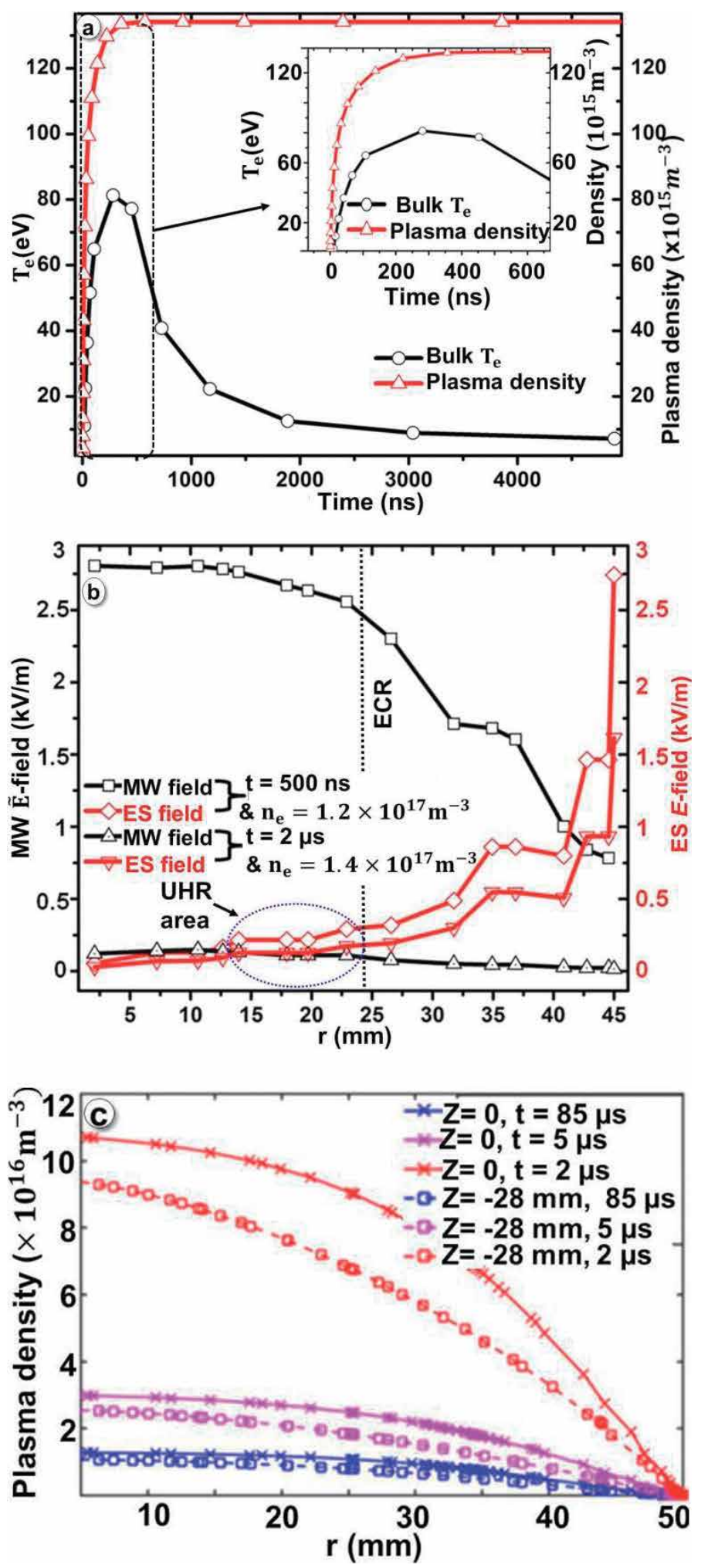

Figure 7.

(a) Temporal evolution of electron density and temperature during plasma formation time at point $(r=0$,

$z=-28 \mathrm{~mm}$ ) with gas pressure, $2 \times 10^{-3}$ mbar. (b) Temporal variation of $M W$ electric field and plasma generated ambipolar electric field, the two main constituents of total electric field. (c) Radial profile of plasma density at three different time instants $(t=\sim 2 \mu s, \sim 5 \mu s$ and $85 \mu \mathrm{s})$, at two axial locations during evolution of the plasma. 
Correspondingly, the plasma density also reaches above the critical density. The decrease in the plasma bulk temperature and the corresponding increase of the plasma density even above the critical density are attributed to be occurring from the off-ECR or ES surface wave heating mechanisms $[29,30]$. In other words, although the plasma density is approaching steady state (Figure 7a), the temperature is not yet stabilized during that time.

To confirm the off-resonance or electrostatic heating methods as discussed above, the evolution of the MW electric field and the electrostatic electric field is shown simultaneously in Figure 7(b). The radial distribution of the two types of the electric field is given in that figure for two discrete time instants and the corresponding plasma densities. One can observe that the MW electric field is significant throughout the cavity and is more than the electrostatic electric field for the time, $t=500 \mathrm{~ns}$. For another instance, $\mathrm{t}=2 \mu \mathrm{s}$, the electrostatic electric field becomes more than the MW electric field throughout the cavity. The electrostatic field is even more in the upper hybrid resonance locations than the MW field as shown in Figure 7(b). This evidence confirms that the electrostatic heating is being taking place at the UHR region where the magnetic field and plasma density satisfy the above-mentioned conditions [28]. To visualize the plasma density pattern due to these electric field behaviors, the radial distribution of the density is shown in Figure 7(c) for different time instances (i.e., 2, 5 and $85 \mu \mathrm{s}$ ) and axial planes on the cylindrical cavity during the plasma evolution. The plane $\mathrm{z}=-28 \mathrm{~mm}$ on the cavity is situated near the MW launching port. Figure 7 (c) shows the plasma density to be more at the central location $(\mathrm{z}=0 \mathrm{~mm})$ than the location $\mathrm{z}=-28 \mathrm{~mm}$ that is located toward the MW launch side.

From the above-mentioned results (Figures 6 and 7), it can be commented that the power is absorbed by the ECR mechanism especially in the plasma condition where the density is below (underdense plasma) the critical density and slightly above the critical density (overdense plasma). If one notices the plasma parameters for the underdense conditions, one can observe that the density remains below the critical density from the time, $\mathrm{t}=\mathrm{t}=45 \mathrm{~ns}$ to $\mathrm{t}=110 \mathrm{~ns}$. In this case, the plasma electrons are magnetized and hence are following the magnetic field lines. The field free zones that are located near $(r, z)=(0,0)$ are being filled by the plasma particles because of the diffusion processes. Due to the ECR heating, the electron temperature is being increased in the field free zones in the underdense plasma situation, $t$ $<110 \mathrm{~ns}$. As the magnetic field lines are stronger $(\mathrm{B} \sim 2300-2600 \mathrm{G})$ near the radial locations of the cavity, i.e., in the gaps of the two pairs of ring magnets, the plasma bulk electron temperature exhibits a sharp gradient in those regions. The maximum plasma bulk electron temperature achievable is $\sim 85 \mathrm{eV}$ that occurs during the time, $\mathrm{t}=280 \mathrm{~ns}$. The high energy part of the plasma bulk electrons is being concentrated completely in the same gap as mentioned before during this time.

It is observed that the plasma bulk electron temperature increases in the radial direction at the regions mainly in between the two pairs of the ring magnets with the increase in time from $\mathrm{t}=280 \mathrm{~ns}$ to $730 \mathrm{~ns}$ [22]. Therefore, it can be summarized that with the increase in plasma density (or time, $t=\sim 45 \mathrm{~ns}$ to $\mathrm{t}=\sim 280 \mathrm{~ns}$ ) from underdense to overdense state, the plasma bulk temperature is increased by an amount of $\sim 80 \mathrm{eV}$ mainly in the radial direction near the region, $24 \mathrm{~mm}<\mathrm{r}$ $<40 \mathrm{~mm},-25 \mathrm{~mm}<\mathrm{z}<25 \mathrm{~mm}$. This is because the ECR surfaces lie in those regions. The continuous heating through ECR in this location causes the high energy part of the plasma electron temperature to be concentrated on the same location even in the overdense plasma state during the time $\mathrm{t}=730 \mathrm{~ns}$.

The anisotropic behavior of the plasma bulk electron temperature even in the overdense plasma signifies the ECR heating [31, 32]. With further increase of time after the MW launch, i.e., near $t=2000 \mathrm{~ns}$, the plasma bulk temperature $\left(\mathrm{T}_{\mathrm{e}}\right)$ is 
decreased to $\sim 10-12 \mathrm{eV}$. This range lies close to the thermal electron temperature. Therefore, the reduction of the high energy component of the plasma bulk temperature can be argued as the minimal occurrence of ECR heating process and correspondingly the initiation of the electrostatic heating mechanisms in the same time frame [29-31, 33]. The plasma reaches steady state after $2000 \mathrm{~ns}(2 \mu \mathrm{s})$ (Figure 7a). At the saturation, the plasma density is approximately 2 times more $(\sim 1.3 \times$ $10^{17} \mathrm{~m}^{-3}$ ) than the critical density. During the same instant of time, the plasma electron temperature and its gradient reduce in the location where the magnetic field is relatively lower.

In order to investigate the different coupling mechanisms involved during the plasma evolution process and their impacts on the plasma parameters, the behavioral pattern of the different components of the electric field $\left(E_{\mathrm{r}}\right.$ and $\left.E_{\mathrm{z}}\right)$ is shown in Figures 8-10. The power coupling mechanisms are ECR, UHR and polarity reversal related to ES heating. The particular type of coupling mechanism is understood from the electric field evolution pattern throughout the cavity. The electric field characters can be useful to characterize the plasma parameters based on the theoretical and experimental proofs. The proofs say the higher the plasma density, the more the power absorbed by the plasma.

\section{3 $\mathrm{E}_{\mathrm{r}}$-field evolution with time}

In Figure 8(a-c), the radial electric field $\left(E_{\mathrm{r}}\right)$ variations are shown for three different z-planes of the cylindrical cavity. The figures also show the evolution of the $E_{r}$-field profile at different time instances after the MW launching. Figure 8(a) gives the $E_{r}$ profile close to the ECR surface $(z=-28 \mathrm{~mm})$, which is also near to the $\mathrm{MW}$ launch point $(\mathrm{z}=-60 \mathrm{~mm})$. Another figure (Figure 8(b)) shows the $\mathrm{E}_{\mathrm{r}}$-field profile along the central plane $(z=0)$ of the cavity, whereas Figure 8(c) gives the $\mathrm{E}_{\mathrm{r}}$-field evolution along the plane $\mathrm{z}=28 \mathrm{~mm}$ that is close to the ion beam extraction point.

In all the figures, a strong inhomogeneity in the $\mathrm{E}_{\mathrm{r}}$-field profile signifies the power absorption locations. Taking a time instant $\mathrm{t}=67 \mathrm{~ns}$ in all the figures, the presence of the $E_{\mathrm{r}}$-field throughout the cavity can be visualized since the plasma density at this time has not reached above the critical density. As the plasma density crosses the critical density with advancement of time ( $>500 \mathrm{~ns}$ ), the $\mathrm{E}_{\mathrm{r}}$-field profile is being modified following the density evolution pattern. The inhomogeneity in the $\mathrm{E}_{\mathrm{r}}$-field profile is more pronounced in Figure 8(b) than in the case of other figures. The difference in the radial variation of the $\mathrm{E}_{\mathrm{r}}$-field comes from the variation of resonance magnetic field contour as shown in Figure 3. The strong inhomogeneous part of $E_{\mathrm{r}}$-field initially very near the ECR surface indicates that the electrons are accelerated by the ECR phenomenon. Then after $>2 \mu \mathrm{s}$, this inhomogeneous part gets shifted toward the UHR $\left(\mathrm{f}_{\mathrm{UHR}}=\sqrt{\left(\mathrm{f}_{\mathrm{pe}}{ }^{2}+\mathrm{f}_{\mathrm{ce}}{ }^{2}\right)}\right)$ regions where the MW

frequency matches the $\mathrm{f}^{\mathrm{UHR}}$. The $\mathrm{E}_{\mathrm{r}}$-field value after $\sim 2 \mu \mathrm{s}$ at $\mathrm{r}=0 \mathrm{~mm}$ and $\mathrm{z}=-28 \mathrm{~mm}$ location is negligible and so the plasma density reached overdense state, whereas for some regions (UHR regions), as shown in Figure 8(a), the $E_{r}$ field component is showing significant intensity. The high value of $E_{\mathrm{r}}$-field, near the location $\left(\mathrm{B}<B_{E C R}\right)$, represents that the plasma is still underdense in this region during time $\mathrm{t}=\sim 3 \mu \mathrm{s}$.

Therefore, the dual conditions $\left(B<B_{E C R}\right.$ and $\left.n_{e}<n_{\text {crit }}\right)$ are satisfied for the UHR heating process. This phenomenon is also being reflected in Figure 7 (a) and (b) as discussed earlier. Figure 7(b) shows the electrostatic electric field to grow with the plasma density and correspondingly the MW electric field decreases after 

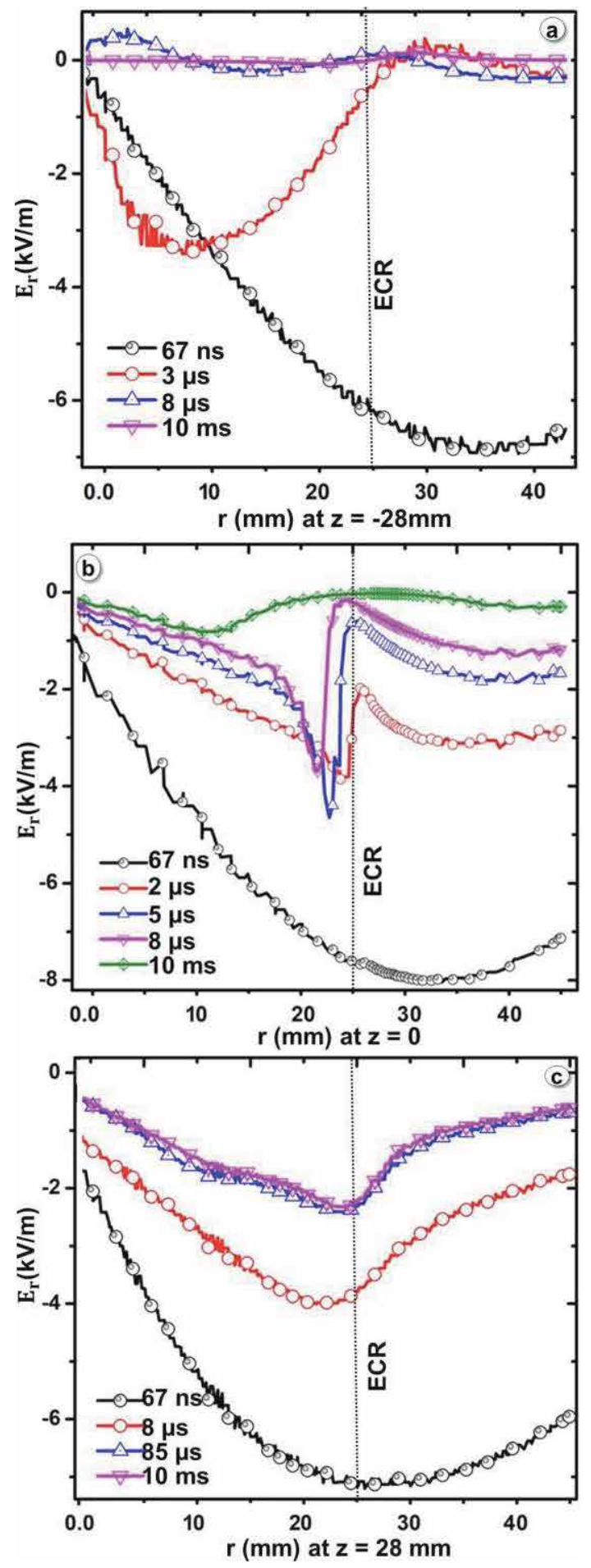

Figure 8.

$\mathrm{E}_{\mathrm{r}}$-field evolution and its pattern during plasma generation $(a) r=-28 \mathrm{~mm},(b) r=0 \mathrm{~mm}$ and $(c) r=28 \mathrm{~mm}$.

500 ns. The electrostatic electric field, the component of the total electric field, is getting increased due to the increase of the plasma ambipolar potential. So this gives a clear evidence of power transferring from MW coupling to the electrostatic wave coupling to the plasma. Since the electrostatic wave does not face any density 


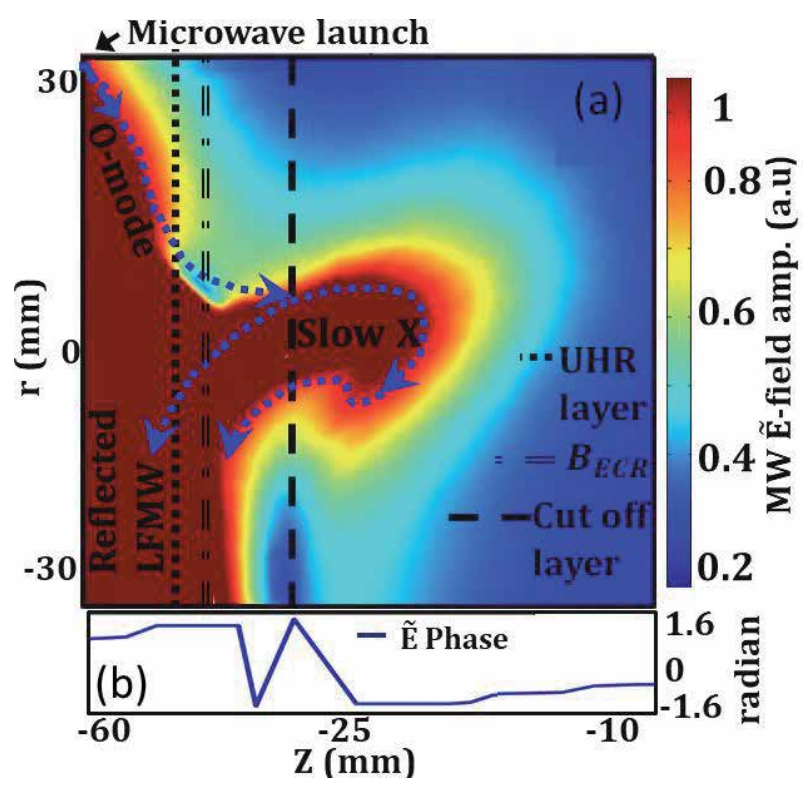

Figure 9.

(a) Propagation and mode conversion of $M W \tilde{\mathrm{E}}$-field corresponding to an ordinary (O)-type resonant mode are shown and $(b)$ Phase angle $\left(\approx \tan ^{-1}(\operatorname{Im}(\tilde{E}) / \operatorname{Re}(\tilde{E}))\right)$ of $M W \tilde{\mathrm{E}}$-field.
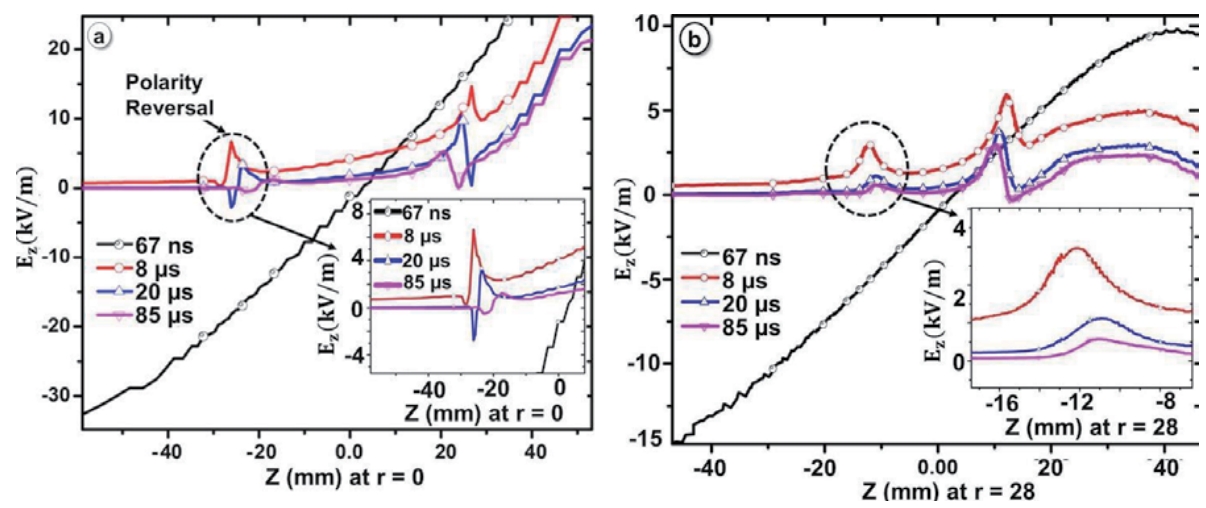

Figure 10.

Spatio-temporal evolution of $E_{z}$-field profile obtained along the axis of the cylindrical cavity. A sharp change in the two places for both the figures implies the power absorption locations that can be checked with Figure 5 described above. (a) $r=0 \mathrm{~mm},(b) r=28 \mathrm{~mm}$.

barrier, it is capable of penetrating the core dense plasma and transferring the energy through damping to the plasma particles. As demonstrated by other groups $[23,28]$, this change in the power coupling mode from the electromagnetic to electrostatic case is a signature of the UHR heating. Due to the heating at UHR, the plasma electron temperature and also the density are possible to enhance further.

It can be observed in Figure 8 that the magnitude of the $E_{r}$-field at time $t=67 \mathrm{~ns}$ (Figure 8) is greater than $7 \mathrm{kV} / \mathrm{m}$ along different z-planes within the cavity near the ECR locations. This intensity is sufficient for creating ionization in the Argon gaseous particles. In the later instant $(\sim 2 \mu \mathrm{s})$ of time, the magnitude of $\mathrm{E}_{\mathrm{r}}$-field changes in between $3.75 \mathrm{kV} / \mathrm{m}$ and $0.15 \mathrm{kV} / \mathrm{m}$ on the ECR regions. This type of nonlinearity in the $\mathrm{E}_{\mathrm{r}}$-field has also been demonstrated by Hopwood et al. [4] before. The power coupling phenomena involved during the plasma evolution can 
also be transmitted into the extracted ion beam performance in terms of spike or instabilities. It was experimentally demonstrated by Ropponen et al. [1] that a sharp transient in the ion current density during the preglow mode can cause a sharp fall in the high energy component of the plasma electron temperature.

Since the inhomogeneous part of $\mathrm{E}_{\mathrm{r}}$-field is shifting toward the off-resonance locations as shown in Figure 8(b), it can also produce high-energy electrons there if certain conditions are satisfied as per Gammino et al. [34]. The conditions say the MW electric field has to be above a certain transition value for being capable to energize the electrons that also depend on the magnetic field gradient present in the same location.

The generated high-energy electrons can interact with the slow extraordinarytype microwave and produce the cyclotron range instability in the plasma [1]. It was also proved experimentally by Mansfeld et al. [35] that the extracted ion beam current from the microwave ion source can gain oscillation due to the presence of cyclotron-type instability of plasma during the afterglow operation mode. The slow extraordinary mode microwave is produced from the mode conversion of ordinarytype microwave near the UHR region. As the mode conversion layer is present in the present plasma cavity, the ordinary mode microwave crosses the evanescent layer and some part of its energy is converted into the slow X mode. Since the plasma is confined in the cavity under mirror magnetic field configuration, the injected MW will have two components, extraordinary mode and ordinary mode.

Figure 9(a) shows the MW ordinary mode is propagating toward the overdense plasma region from the underdense launching point. At some point, it will encounter a cut-off corresponding to the ordinary-type MW. At the cut-off, some part of the ordinary mode MW energy is evanescently transformed into a slow extraordinary mode following the CMA diagram concepts. For that reason, a bend in the MW propagation in the slow extraordinary $(\mathrm{X})$ mode is seen in the electric field simulation (Figure 9b). This slow X mode then propagates toward the UHR region and hence the electric field is being accumulated there, as shown in Figure 9(a). The accumulation of the electric field at this location increases its intensity at some plasma condition and can cross the corresponding parametric decay threshold condition. The parametric decay of the slow X mode near the UHR region can generate ion- and electron-type electrostatic waves as per the literature [36].

As a supportive evidence of the generation of electrostatic ion wave, Figure 8(b) shows that the inhomogeneous part of the $\mathrm{E}_{\mathrm{r}}$-field is shifting toward the offresonance region with a velocity of $1250-1500 \mathrm{~m} / \mathrm{s}$. This range of velocities falls in the range of ion acoustic speed. Based on the published reports [37], the electric field propagating perpendicularly with respect to the external magnetic field as in the present case (Figure 9a) encounters the UHR layer in the overdense plasma state. In the present computation, the ordinary mode ( $\mathrm{O}$ mode) electric field after converting into the slow extraordinary mode ( $\mathrm{X}$ mode) shows a bending in the perpendicular direction and reaches the UHR region. This is known as ' $\mathrm{O}$ - slow $\mathrm{X}$ ' conversion process that is responsible for generating ion waves and makes the inhomogeneous electric field to shift at the same ion acoustic speed [38].

\section{4 $E_{z}$-field evolution in plasma with time}

The spatio-temporal evolution of the axial component $\left(\mathrm{E}_{\mathrm{z}}\right)$ of the electric field is shown in Figure 10 throughout the cylindrical cavity along the planes $\mathrm{r}=0 \mathrm{~mm}$ and $28 \mathrm{~mm}$, respectively. It is seen that only a portion of single wavelength of the wave electric field is present that has significant intensity throughout the plasma cavity after the immediate instant (67 ns) of MW launching. The magnitude of the $\mathrm{E}_{\mathrm{z}}$ field becomes almost zero throughout the cavity as soon as the plasma starts creating 
after the MW launching. Therefore, the $\mathrm{E}_{\mathrm{z}}$ field does not play any significant role in accelerating the electrons, and if this would happen, the electrons losses will occur in the axial direction. Figure 10(a-b) evidences that the $E_{z}$ field becomes almost zero everywhere except near the ECR surface after $\sim 8 \mu \mathrm{s}$. The $\mathrm{E}_{\mathrm{z}}$-field magnitude shows minimum values where the plasma density is of maximum values.

It can be observed in Figure 10(a) that the polarity in the inhomogeneous part of the electric field is getting opposite for two different time instances, 20 and $85 \mu$ s. The reversal in the polarity of electric field occurs near the ECR surface. The polarity reversal is caused by the ambipolar field produced from the plasma density gradient. The plasma density gradient is computed from the electron momentum equation using drift-diffusion approach in the present FEM model [38]. The shifting of the inhomogeneous part of the electric field is in the inward direction. The speed of displacement of the inhomogeneous part of the electric field is estimated in the range of $\sim 10^{3} \mathrm{~m} / \mathrm{s}$ that lies in the range of ion sound speed. Similar shifting at the same velocity is also observed before in Figure 8(b) corresponding to the $\mathrm{E}_{\mathrm{r}}$-plots. These observations indicate that the plasma density gradient near the ECR surface is accompanied by the generation of ion acoustic waves that are electrostatic in nature $[39,40]$. Hence, the electric field polarity reversal associated with electrostatic ion wave heating is being initiated during this period of the plasma evolution after the microwave launch.

\section{Validation with experiment}

Experiments are performed to cross-check the above-mentioned plasma parameters obtained during the gas ignition moment. The present section of the chapter provides the details of the experimental methods, analysis of the experimental results and also a comparative study of the experimental data with the simulation.

\subsection{Methods of experiment}

Experiments are carried out in a microwave ion source system that has similar system configuration, magnetic field distribution, MW conditions and also the operating conditions. The simulated temporal plasma parameters, such as the plasma density and hot electron temperature, are validated with the experiment [23]. In the present experiment, MW-plasma reactor of the experimental set-up is a cylindrical cavity (Figure 11) of 107-mm length and 88-mm diameter. The plasma in the reactor is generated by coupling microwave through the electron cyclotron resonance (ECR) heating as well as off-ECR heating methods, as discussed before. The complete experimental set-up consists of a cylindrical cavity, microwave system, ion beam extraction system and two pairs of ring magnets (each magnet has pole strength $\sim 1.38 \mathrm{~T}$ ) assembly [23]. The plasma cavity/reactor is surrounded by two pairs of ring magnets to generate a mirror-type magnetic field to confine the plasma inside the cavity.

To generate plasma, the MW is produced by a magnetron (power: $0-2 \mathrm{~kW}$, make: Richardson Electronics, Model no. NL10250-7), which is operated either in continuous or in pulsed mode. MW power is fed to the reactor through a combination of a four-step ridged waveguide, a HV break and vacuum window assembly, an impedance tuner unit, directional coupler and an isolator with water dummy load (Figure 11). The plasma impedance is matched by a 3-stub tuner to get maximum E-field at the center of the MW-plasma reactor. MW is coupled to the cylindrical plasma reactor by a four-step ridged waveguide (WR 284) [23]. The 


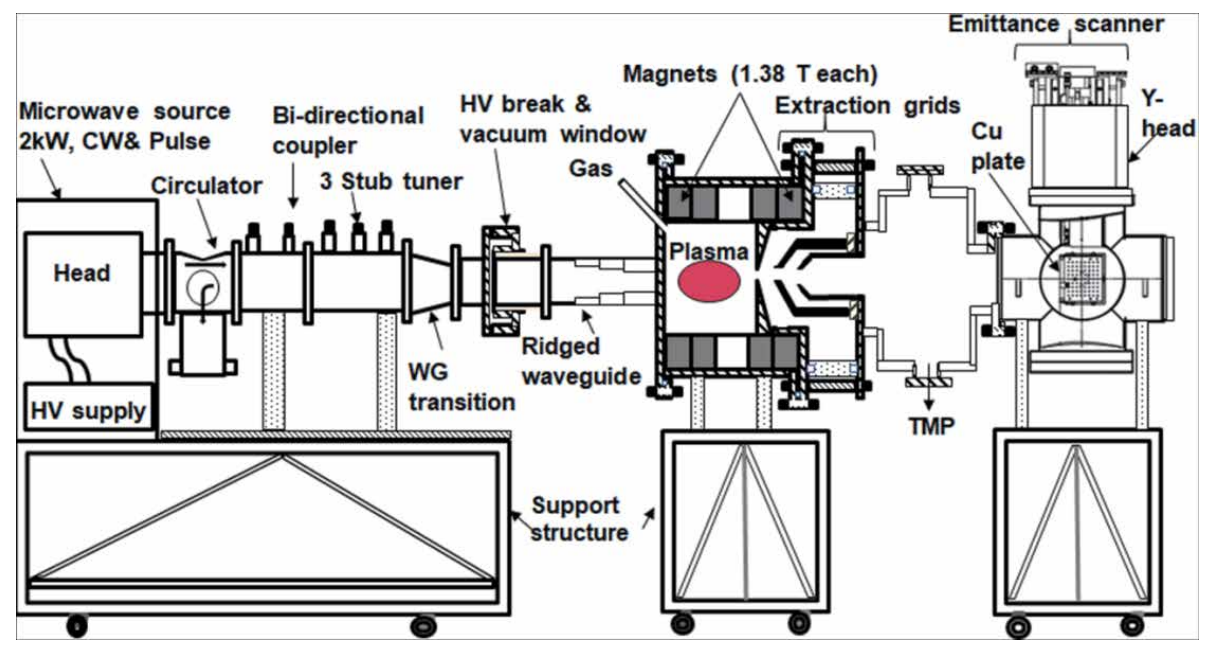

Figure 11.

Schematic view of the experimental system.

impedance of WR284 waveguide (four-step ridge waveguide) having 220-mm length is made comparable to the typical plasma impedance by embedding four ridge sections on both the inner side walls of it. The ridge sections of different lengths but same width $(48 \mathrm{~mm})$ are placed consecutively at the broader walls of the waveguide. As shown in Figure 11, the ridge waveguide is mechanically connected to one side of a high voltage (HV) break cum vacuum window, made of Teflon having a dimension of $35 \mathrm{~mm}$ diameter and $6 \mathrm{~mm}$ thickness, whose other side is connected to the ion source cavity (Figure 11). The other side of the cavity is connected to the conventional pierce geometry-based 3-grid extraction system, housed in a drift duct vacuum chamber that consists of a plasma grid, an extraction grid and a grounded grid (Figure 11). The ion source is evacuated by a combination of Turbo Molecular Pump, which is backed by a dry-scroll rotary pump, connected to the drift duct vacuum chamber in the downstream side of the extraction system. The gas feed system comprises a needle valve, mass flow controller and the required gas cylinder. In the present experiment, nitrogen gas is used because of availability. Other gases, like argon or hydrogen, can also be used. The experiment is carried out for the MW power in the range of 50-700 W and gas pressure in the range of $1 \times 10^{-3}$ mbar to $1 \times 10^{-4}$ mbar. The Langmuir probe (LP) diagnostics is used to characterize the plasma parameters within the pressure range varying from $2 \times$ $10^{-4}$ to $1 \times 10^{-3}$ mbar [23].

All the MW power ranges mentioned throughout the chapter are considered as set power. The difference of forward and reflected power is considered to be plasma-absorbed power. MW reflection varies from 5 to $10 \%$ within the above set power range. These ranges of plasma reflection with similar experimental set-ups and operating environments are reported in [10]. The accuracy at low set power levels of magnetron is tested by repeated measurements of its output power (forward) before the experiment is performed. An extra component, named isolator with water-cooled dummy load (make: National Electronics, Model: 2722-16210311, isolation: $26 \mathrm{~dB}$, reflection rating: $6.5 \mathrm{~kW}$ ) is placed in the experimental setup (not shown in Figure 11) before the HV break. The power and carrier frequency signals are measured by microwave spectrum analyzer (model: FSH8, make:

ROHDE \& SCHWARZ, band: $100 \mathrm{~Hz}-8 \mathrm{GHz}$ ) at the directional coupler port.

Signals are attenuated by $60 \mathrm{~dB}\left(\equiv 10^{6}\right.$, power ratio) at the directional coupler ports 
before coming to microwave spectrum analyzer through the high frequency (0-40 GHz), low loss and low VSWR cable of length $\sim 1 \mathrm{~m}$.

To generate plasma in the low power range, magnetron's low power testing is required. In the present experiment, low power testing ensures the variation of full width at half maximum of $2.45 \mathrm{GHz}$ frequency is within 5-12 $\mathrm{MHz}$ (within the specified $\pm 25 \mathrm{MHz}$ bandwidth as per Sairem data). Also, the set power fluctuations are within 2-5\% as shown in Figure 12(a), which is considered to be stable operating conditions. After performing the inverse Fourier transform of the MW spectrum analyzer data, a graph of detected MW power vs. time is shown in Figure 12 (b) to verify the power levels at the first few milliseconds for the comparison of plasma parameters with the simulated data. The rise time of the pulse is obtained as $\sim 2.2 \mu \mathrm{s}$.

The magnetron's output (set power) in the low power range is checked in time scale prior to the Langmuir probe diagnostic [23]. The response of the magnetron set power at $2.45 \mathrm{GHz}$ frequency is recorded at the directional coupler port by a microwave spectrum analyzer (SA) circuitry. The circuitry consists of a high frequency cable, a band pass filter, and spectrum analyzer and FSH4 View software.
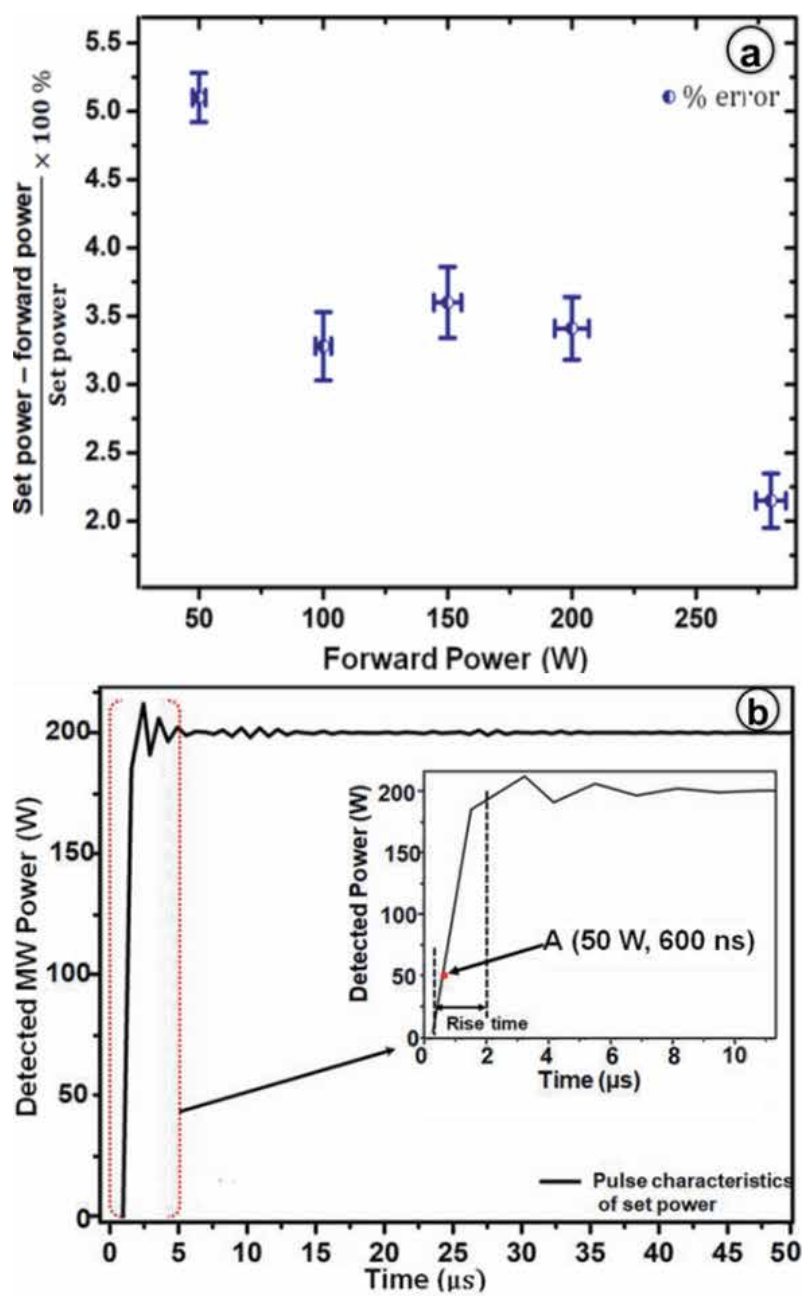

Figure 12.

(a) Percentage error of the magnetron set power fluctuations. Data for 280 W have been benchmarked with the Sairem company data and $(b)$ variation of the detected MW power vs. time for the set power of $200 W$. 
The magnetron's pulse response at a fixed set power, $200 \mathrm{~W}$, is obtained by taking the inverse Fourier transform of the microwave spectrum analyzer data. The magnetron's rise time is $\sim 2.2 \mu \mathrm{s}$. This exercise of measuring the pulse response of a particular set power can help to pick up the temporal values at different set power levels following the pulse response of magnetron [23].

To compare the simulated hot electron temperature and density, their parameters are noted down at different instant of time during the plasma evolution. Taking the time instant to be same as that of the simulation, the set power values are noted down in an experimental datasheet. Then, a single Langmuir probe measures the plasma floating potential at the noted set power values as mentioned above [23]. The Langmuir probe measurement is performed in steady state plasma condition. Since the real-time measurement of the plasma parameter requires in the ns-time scale very sophisticated and expensive hardware that has faster time responses (ns range), steady state Langmuir probe measurements are performed to avoid those expensive diagnostics.

\subsection{Comparison of experimental results with simulation}

The line plots are shown in Figure 13(a) for the simulated hot $\mathrm{T}_{\mathrm{e}}$. The simulated data point lies in a region that is very close to the experimental data points. The simulated hot $\mathrm{T}_{\mathrm{e}}$ is approximately $78 \mathrm{eV}$ at $70 \mathrm{~W}$ of plasma absorbed power during time $t=600 \mathrm{~ns}$. One can also observe that the set power for the magnetron reaches $50 \mathrm{~W}$ at the same time instant. The measurement of the hot $\mathrm{T}_{\mathrm{e}}$ by a Langmuir probe diagnostic at the same set power level shows it to be $\sim 72 \mathrm{eV}$. Similarly, the hot $\mathrm{T}_{\mathrm{e}}$ was measured for two other power levels, 70 and $130 \mathrm{~W}$. The hot $\mathrm{T}_{\mathrm{e}}$ at these two power levels are $\sim 36$ and $28 \mathrm{eV}$, respectively. Hence, the experimental results fit well with the simulated values described above [23]. On the other hand, the experimental data for the plasma density are also shown in Figure 13(b). The plasma densities for the above set power levels are observed to be $\sim 1.8-2$ times less than the simulated data. The deviation of the measured plasma density from the simulated values is caused due to the difference in the absorbed power that is absorbed by the plasma in both the cases (experiment and simulation). The experimental results for the plasma density are shown in Figure 13(b) for three different set powers. The measured plasma density $\left(\sim 1.1 \times 10^{17} \mathrm{~m}^{-3}\right)$ at set power of $200 \mathrm{~W}$ agrees well with the simulated plasma density $\left(\sim 1.3 \times 10^{17} \mathrm{~m}^{-3}\right)$.
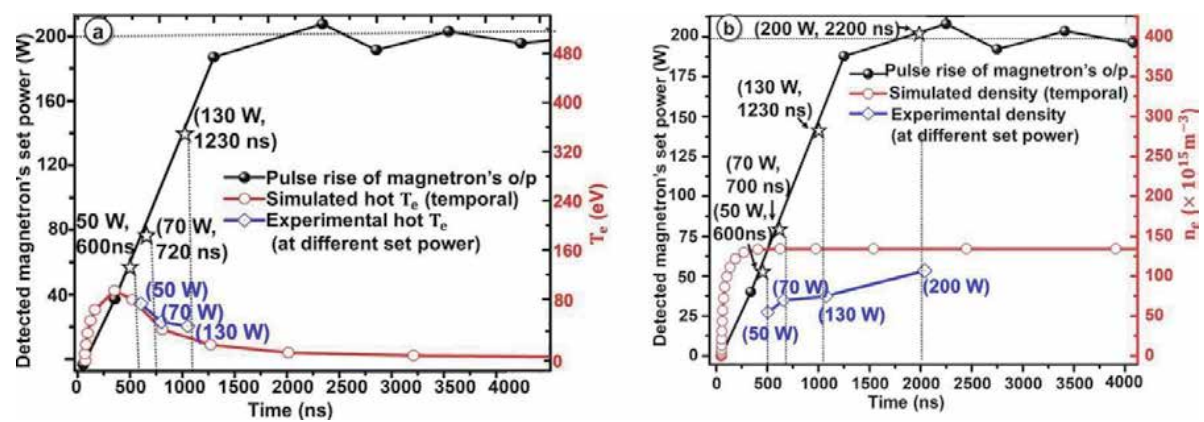

Figure 13.

(a) Simulated temporal hot $\mathrm{T}_{\mathrm{e}}$ variation along with experimental data, taken at discrete set power values. Plasma-absorbed power during simulated hot $\mathrm{T}_{\mathrm{e}}$ evolution is fixed at $70 \mathrm{~W}$. Experimental set powers 50,70 and $130 \mathrm{~W}$ correspond to plasma-absorbed powers at 40, 50 and $70 \mathrm{~W}$, respectively [14]. Pulse rise of set power is also shown to verify the power levels during the ns to few $\mu$ s periods. (b) Simulated temporal plasma density variation along with experimental results, obtained at the same set power values. 


\section{Conclusion}

The evolution of the spatial and temporal plasma parameters are presented in the current chapter during the gas ignition process made by injecting the $2.45 \mathrm{GHz}$ microwave to an ion source cavity. The simulated results supported by the experiment confirm that the plasma parameters are influenced significantly by the electric fields during the plasma ignition period. Due to the shifting of the microwave coupling mechanisms to the plasma, the plasma density and the hot fraction of the electron temperature are also getting affected during the plasma generation period. The initial rise of the hot fraction of the plasma electron temperature from the start of microwave interaction into the plasma is argued to be caused by the electron cyclotron resonance heating phenomenon. After certain instant of microwave launch, the slight increase in the plasma density and the decrease in the plasma electron temperature are proved to be happening from the electrostatic heating mechanism. The electrostatic heating near the upper hybrid resonance region causes to shift the inhomogeneous part of the electric field at the velocity of the ion acoustic speed when the plasma density reaches above the critical density corresponding to the launch microwave, $2.45 \mathrm{GHz}$.

The experimental plasma parameters are obtained in an experimental set-up that has similar system configuration and the operating environment as that of the simulation. The comparison shows a reasonable agreement with the simulated results. The plasma density especially in the overdense plasma condition is found to be agreeing more in the overdense plasma than in the underdense plasma condition. During plasma evolution after the microwave launch, the microwave coupling mechanisms are modified following the corresponding electric field (electrostatic and electromagnetic) distribution pattern throughout the ion source cavity. Initially, the electron cyclotron resonance heating comes into play to ionize the gaseous particle and generate plasma that contains the maximum fraction of the highenergy electrons in the ion source plasma. Then, as the density reaches in the overdense condition, the coupling mechanisms are the electrostatic wave heating in the ion acoustic frequency range. As the electrostatic wave does not suffer any density cut-off, the density is increased further above the critical density. Simultaneously, the polarity of the axial electric field is reversed near the electron cyclotron resonance region signifying the creation of the plasma density gradient due to the generation of strong ambipolar electric field near the resonance region. In future, it is intended to study the different power coupling mechanisms in the overdense plasma state that are caused due to the heating at the cyclotron harmonics by the generated electrostatic waves in the present experimental device. 


\section{Author details}

Chinmoy Mallick ${ }^{1,2 *}$, Mainak Bandyopadhyay ${ }^{1,2}$ and Rajesh Kumar $^{1,2}$

1 Institute for Plasma Research (IPR), Gandhinagar, Gujarat, India

2 Homi Bhabha National Institute (HBNI), Mumbai, Maharashtra, India

*Address all correspondence to: chinmoyju1990@gmail.com

\section{IntechOpen}

(C) 2020 The Author(s). Licensee IntechOpen. This chapter is distributed under the terms of the Creative Commons Attribution License (http://creativecommons.org/licenses/ by/3.0), which permits unrestricted use, distribution, and reproduction in any medium, provided the original work is properly cited. (cc) BY 


\section{References}

[1] Ropponen T, Tarvainen O, Izotov I, Noland J, Toivanen V, Machicoan G, et al. Studies of plasma breakdown and electron heating on a $14 \mathrm{GHz}$ ECR ion source through measurement of plasma bremsstrahlung. Plasma Sources Science and Technology. 2011;20:055007. DOI: 10.1088/0963-0252/20/5/055007

[2] Cortázar OD, Megía-Macías A, Vizcaíno-de-Julián A. Experimental study of breakdown time in a pulsed 2.45-GHz ECR hydrogen plasma reactor. IEEE Transactions on Plasma Science. 2012;40:3409. DOI: 10.1063/ 1.4819875

[3] Cortazar OD, Megía-Macías A, Tarvainen O, Vizcaíno-de-Julián A, Koivisto $\mathrm{H}$. Plasma distributions observed in a $2.45 \mathrm{GHz}$ hydrogen discharge. Plasma Sources Science and Technology. 2014;23:065028. DOI: 10.1088/0963-0252/23/6/065028

[4] Hopwood J, Wagner R, Reinhard DK, Asmussen J. Electric fields in a microwave-cavity electroncyclotron-resonant plasma source. Journal of Vacuum Science and Technology A. 1990;8:2904. DOI: 10.1116/1.576647

[5] Stenzel RL. A new probe for measuring small electric fields in plasmas. The Review of Scientific Instruments. 1991;62:130. DOI: 10.1063/ 1.1142514

[6] Celona L, Gammino S, Ciavola G, Maimone F, Mascali D. Microwave to plasma coupling in electron cyclotron resonance and microwave ion sources. Review of Scientific Instruments. 2010; 81:02A333. DOI: 10.1063/1.3265366

[7] Consoli F, Celona L, Ciavola G, Gammino S, Maimone F, Barbarino S, et al. Influence of microwave driver coupling design on plasma density at Testbench for Ion sources Plasma Studies, a
2.45 GHz electron cyclotron resonance plasma reactor. Review of Scientific Instruments. 2008;79:02A308. DOI: $10.1063 / 1.2805665$

[8] Takahashi K, Kaneko T, Hatakeyama R. Polarization-reversalinduced damping of left-hand polarized wave on electron cyclotron resonance. Physical Review Letters. 2005;94: 215001. DOI: $10.1103 /$

PhysRevLett.94.215001

[9] Becker MM, Kählert H, Sun A, Bonitz M, Loffhagen D. Advanced fluid modeling and PIC/MCC simulations of low-pressure ccrf discharges. Plasma Sources Science and Technology. 2017; 26:044001. DOI: 10.1088/1361-6595/ aa5cce

[10] Hagelaar GJM, Makasheva K, Garrigues L, Boeuf JP. Modelling of a dipolar microwave plasma sustained by electron cyclotron resonance. Journal of Physics D: Applied Physics. 2009;42:

194019. DOI: $10.1088 / 0022-3727 / 42 / 19 /$ 194019

[11] COMSOL Multiphysics Reference Manual, Version 5.2. COMSOL, Inc. Available from: www.comsol.com

[12] Eric J. Vanzura et al, The NIST 60 Millimeter Diameters Cylindrical Cavity Resonator: Performance Evaluation for Permittivity Measurements', NIST Technical Note 1354. 1993

[13] Celona L et al. Observations of resonant modes formation in microwave generated magnetized plasmas. European Physical Journal D: Atomic, Molecular, Optical and Plasma Physics. 2011;61:107-115

[14] McDonald KT. An Electrostatic Wave. arXiv:physics/0312025

[15] Chen FF. Introduction to the Plasma Physics and Controlled Fusion: Plasma 
Physics. UK: London Press, London; 1986. Addison-Wesley; 1994

[16] Muller A et al. On the charge state distribution of multiply charged ions extracted from electron beam ion sources. Nuclear Instruments and Methods. 1977;140:181

[17] Stix TA. Waves in Plasmas. New York: Springer-Verlag; 1992

[18] Mascali D. A new approach to the study of the ECR heating and particle dynamics in the plasma of electron cyclotron ion sources [PhD thesis]. UniversitA degli studi di Catania; 2008

[19] Castro G et al. Study of innovative plasma heating methods and applications to high current ion sources [PhD thesis]. Universita degli Studi di Catania;

[20] Castro G et al. Experimental investigation of non-linear wave to plasma interaction in a quasi-flat magnetostatic field. Review of Scientific Instruments. 2016;87:02A507

[21] Stix TH, Swanson DG. Propagation and mode conversion for waves in nonuniform plasmas. In: Galeev A,

Sudan RN, editors. Plasma Physics Lab. Rep. PPPL-1903 (June 1982); Handbook of Plasma Physics. Vol. 1. North Holland, Amsterdam: Princeton University; (to be published)

[22] Mallick C, Bandyopadhyay M, Kumar R. Spatio-temporal evolution of electric field inside a microwave discharge plasma during initial phase of ignition and its effect on power coupling. Physics of Plasmas. 2019;26: 043507. DOI: $10.1063 / 1.5085204$

[23] Mallick C, Bandyopadhyay M, Kumar R. Plasma characterization of a microwave discharge ion source with mirror magnetic field configuration. The Review of Scientific Instruments. 2018;89:125112. DOI: 10.1063/1.5048292
[24] Kinder RL, Kushner MJ. Wave propagation and power deposition in magnetically enhanced inductively coupled and helicon plasma sources. Journal of Vacuum Science and Technology A. 1999;17:2421. DOI: 10.1116/1.581978

[25] Mascali D, Celona L, Gammino S, Miracoli R, Castro G, Gambino N, et al. Electrostatic wave heating and possible formation of self-generated high electric fields in a magnetized plasma. Nuclear Instruments and Methods in Physics Research A. 2011;653:11-16. DOI: 10.1016/j.nima.2011.02.04

[26] You HJ, Meyer FW, Chung KS. The cold and hot electron populations, temperatures and their transports in the edge plasma of the ORNL CAPRICE ECR ion source. Plasma Sources Science and Technology. 2009;18: 015004. DOI: 10.1088/0963-0252/18/1/ 015004

[27] Chen L, Zhao Y, Wu K, Wang Q, Meng Y, Ren Z. On the generation of magnetic field enhanced microwave plasma line. Physics of Plasmas. 2016;23: 123509. DOI: $10.1063 / 1.4971446$

[28] Mascali D, Celona L, Gammino S, Castro G, Miracoli R, Romano FP, et al. An investigation on the formation of suprathermal electrons in a B-min ECR machine and a novel method for their damping. Plasma Sources Science and Technology. 2013;22:065006. DOI: 10.1088/0963-0252/22/6/065006

[29] Sugai H, Ghanashev I, Mizuno K. Transition of electron heating mode in a planar microwave discharge at low pressures. Applied Physics Letters. 2000;77:3523. DOI: 10.1063/1.1329322

[30] Shalashov AG, Viktorov ME, Mansfeld DA, Golubev SV. Kinetic instabilities in a mirror-confined plasma sustained by high-power microwave radiation. Physics of Plasmas. 2017;24: 032111. DOI: $10.1063 / 1.4978565$ 
[31] Bodendorfer M, Altwegg K, Wurz P, Shea $\mathrm{H}$. Field structure and electron life times in the MEFISTO electron cyclotron resonance ion source. Nuclear Instruments and Methods in Physics Research B. 2008;266:4788. DOI: 10.1016/j.nimb.2008.07.019

[32] Izotov I, Tarvainen O, Mansfeld D, Skalyga V, Koivisto H, Kalvas T, et al. Microwave emission related to cyclotron instabilities in a minimum-B electron cyclotron resonance ion source plasma. Plasma Sources Science and Technology. 2015;24:045017. DOI: 10.1088/0963-0252/24/4/045017

[33] Chelvam PP, Raja LL. Modeling of gas breakdown and early transients of plasma evolution in cylindrical alldielectric resonators. Journal of Physics D: Applied Physics. 2017;50:474003. DOI: $10.1088 / 1361-6463 /$ aa925f

[34] Gammino S, Mascali D, Celona L, Maimone F, Ciavola G. Considerations on the role of the magnetic field gradient in ECR ion sources and buildup of hot electron component. Plasma Sources Science and Technology. 2009; 18:045016. DOI: 10.1088/0963-0252/18/ 4/045016

[35] Mansfeld D, Izotov I, Skalyga V, Tarvainen O, Kalvas T, Koivisto H, et al. Dynamic regimes of cyclotron instability in the afterglow mode of minimum-B electron cyclotron resonance ion source plasma. Plasma Physics and Controlled Fusion. 2016;58: 045019. DOI: 10.1088/0741-3335/58/4/ 045019

[36] Castro G, Mascali D, Gammino S, Torrisi G, Romano FP, Celona L, et al. Overdense plasma generation in a compact ion source. Plasma Sources Science and Technology. 2017;26: 055019. DOI: 10.1088/1361-6595/aa61c4

[37] Van Niekerk EG, Krumm PH, Alport MJ. Electrostatic ion cyclotron waves driven by a radial electric field.
Plasma Physics and Controlled Fusion. 1991;33:375-388

[38] Schulze J, Donkó Z, Lafleur T, Wilczek S, Brinkmann RP. Spatiotemporal analysis of the electron power absorption in electropositive capacitive RF plasmas based on moments of the Boltzmann equation. Plasma Sources Science and Technology. 2018;27: 055010

[39] Dieckmann ME, Sarri G, Doria D, Ynnerman A, Borghesi M. Particle-incell simulation study of a lower-hybrid shock. Physics of Plasmas. 2016;23: 062111

[40] Fischer P, Gauthereau C, Godiot J, Matthieussent G. Ion acoustic wave generation by a standing electromagnetic field in a sub-critical plasma. Journal de Physique. 1987;48: 233-238 
Section 4

\section{Physics of Plasma Antennas}





\title{
Chapter 4
}

\section{Plasma Antennas}

\author{
Theodore Anderson
}

\begin{abstract}
We have demonstrated that one or two plasma tubes can be used to focus, spread, and steer antenna beams. We have also shown that we can simulate convex and concave plasma lenses by using cylindrical plasma tubes. Focusing by a plasma is useful because it can be used to increase the gain of an antenna, and to quickly reconfigure the beamwidth as needed without physically moving the antenna. With this technology, there is no need for phased arrays to steer and focus an antenna beam. Beam steering with a plasma allows tuning to different frequencies which is a difficult task for standard antennas. Our experimental results with $44 \mathrm{GHz}$ showed a dramatic improvement in beam steering and focusing characteristics compared to beam focusing and steering at $24 \mathrm{GHz}$. The shorter wavelength compared to the spatial variation in plasma density over the radius of the plasma tube, the easier it is to steer and focus antenna beams. These results have been incorporated in a new smart plasma antenna design.
\end{abstract}

Keywords: plasma antenna, antenna beam focusing, antenna beam steering, physics of refraction through a plasma

\section{Introduction}

Plasma antennas use partially or fully ionized gas as the conducting medium instead of metal to create an antenna. The advantages of plasma antennas are that they are highly reconfigurable and can be turned on and off. Hence research to reduce the power required to ionize the gas at various plasma densities is important and this has been achieved by various techniques including pulsing techniques. The power requirements for plasma antenna operation continue to decrease.

The same geometric resonances apply to plasma antennas as metal antennas. Plasma antennas of the same shape, length, and frequency of corresponding metal antennas will have the same radiation patterns. Plasma antennas have the advantage of reconfigurability.

High frequency antennas can transmit and receive through lower frequency plasma antennas eliminating or reducing co-site interference. Because of this principle, higher frequency plasma antennas can be nested inside lower frequency plasma antennas and the higher frequency plasma antennas can transmit and receive through the lower frequency plasma antennas. Higher frequency plasma antenna arrays can transmit and receive through lower frequency plasma antenna arrays. Co-site interference occurs when larger frequency antennas block or partially block the radiation patterns of smaller higher frequency antennas. With plasma antennas, co-site interference can be reduced or eliminated. The interference among plasma antennas can be reduced or eliminated by turning all the plasma antennas off (extinguishing the plasma) except the plasma antennas that are 
transmitting and/or receiving. This is not possible with metal antennas. A general rule is that when an incident electromagnetic wave upon a plasma antenna is such that the frequency of the incident electromagnetic wave is greater than the plasma frequency of the plasma, the incident electromagnetic wave passes through the plasma without attenuation. If the incident electromagnetic wave has a frequency much less than the plasma frequency, the plasma behaves similar to a metal. The frequency at which plasma behaves like a metal or a dielectric is reconfigurable. The plasma frequency is a natural frequency of the plasma and it is a measure of the amount of ionization in the plasma. It is defined and used throughout this book. Both plasma antennas and metal antennas increase in size as the frequencies they operate goes down to maintain geometric resonance and high efficiency. However as the frequency of operation of the plasma antenna decreases, the density of the plasma needed to operate the plasma antenna also goes down. A rule of thumb is that the plasma frequency should be about twice the operating frequency of the plasma antenna. Hence the plasma frequency goes down as the frequency of the plasma antenna goes down. As the plasma frequency decreases, the plasma antenna becomes transparent to a greater bandwidth of electromagnetic waves. In short as the plasma antenna increases in size, the RCS of the plasma antenna goes down whereas for the corresponding metal antenna, the RCS goes up as the metal antenna increases in size. This gives the plasma antenna some great advantages at low frequencies over the corresponding metal antenna. In addition plasma antennas do not receive electromagnetic noise greater than the plasma frequency since these frequencies pass through the plasma antenna.

Related to plasma antennas, plasma frequency selective surfaces, plasma waveguides, and plasma co-axial cables have been developed. Unlike metal frequency selective surfaces, plasma frequency selective surfaces have the properties of reconfigurable filtering of electromagnetic waves. This could have tremendous advantages to radome design. Plasma frequency selective surfaces can be reconfigured by varying the plasma density, varying the shape of the elements, or tuning any number of the plasma FSS elements on or off. Plasma wave guides and plasma co-axial cables can be stealth like plasma antennas, and they can operate at low frequencies, and be invisible at high frequencies. Plasma waveguides and coaxial cables can be feeds for plasma antennas. Plasma feeds as well as the plasma antennas have reconfigurable impedances. If the impedance of the plasma antenna is changed, the impedance of the plasma antenna feeds can be changed to maintain impedance matching.

Thermal noise in a plasma antenna is less than the thermal noise in a metal antenna at the higher frequencies. Higher frequencies mean that there is a point in the RF spectrum in which the thermal noise of plasma antennas is equal to the thermal noise of metal antennas. At higher frequencies than this point, the plasma antenna thermal noise decreases drastically compared to a metal antenna. Below this point the thermal noise of the plasma antenna is greater than a metal antenna. For a fluorescent tube which has been built as a plasma antenna, the point where the thermal noise of the plasma antenna is equal to the metal antenna is about $1 \mathrm{GHz}$. This point can be decreased in frequency by decreasing the plasma density and/or gas pressure. The plasma in the plasma antennas are inert gases that operate at energies and frequencies in which Ramsauer Townsend Effects apply.

Ramsauer Townsend Effects mean that the electrons in the plasma diffract around the ions and neutral atoms in the plasma. This means that the collision rate of the unbound electrons in the plasma with ions and neutral atoms is small and much smaller than in a metal. This phenomenon contributes to the lower thermal noise plasma antennas have over corresponding metal antennas. 
Satellite plasma antennas benefit from the lower thermal noise at the frequencies they operate. Ground based satellite antennas point at space where the thermal noise is about $5^{\circ} \mathrm{K}$. A low thermal noise, high data rate satellite plasma antenna system is possible with low noise plasma feeds and a low noise receiver. Satellite plasma antennas can operate in the reflective or refractive mode. Satellite plasma antennas need not be parabolic but can be flat or conformal and effectively parabolic. The effective plasma parabolic dish antenna is part of the scope of future work. Electromagnetic waves reflecting off of a bank of plasma tubes get phase shifted as a function of the plasma density in the tube. This becomes an effective phase array except that the phase shifts are determined by the plasma density. If the plasma density in the tubes is computer controlled, the reflected beam can be steered or focused even when the bank of tubes is flat or conformal. In the refractive mode, the refraction of electromagnetic waves depends upon the density of the plasma. In the refractive mode, steering and focusing can be computer controlled even when the bank of tubes is flat. This eliminates the problem of the blind spot and feed losses caused by the feed horn and receiver in front of a metal satellite antenna.

Pulsing techniques instead of applying continuous energy were developed to increase the plasma density and decrease the amount of energy to maintain the plasma.

In the history of antennas, it has been difficult to develop low frequency directional and electronically steerable antennas that fit on land vehicles and aircraft. Low frequency means the wavelength is on the order or larger than the vehicle. With plasma antennas this is possible with multipole expansions of clusters of plasma antennas that are all within a wavelength of each other. This depends on the ability of turning plasma antennas on or off (extinguishing the plasma) to create reconfigurable multipoles of plasma antennas that can be rotated in time creating directional and steerable antenna beams. This is not possible with metal antennas because they cannot be turned on and off.

Several groups have done work in using numerical techniques to plot plasma antenna radiation patterns. Zhou et al. [1] used FDTD Method techniques. Bogachev et al. [2] predicted radiation patterns for plasma asymmetrical dipole antenna. Zhivko Kiss'ovski [3] calculated the radiation pattern of miniaturized plasma antennas. Golazari et al. [4] did measurements and simulations of a loop plasma antenna in UHF band Barro et al. [5] did simulations to get the radiation patterns of cylindrical plasma antennas. Kumar et al. [6] have done simulations of a plasma antenna array. Melazzi et al. [7] have developed a plasma antenna numerical code called ADAMANT. An overview of experimental and numerical research is Anderson et al. [8]. Mansutti et al. [9] have done numerical work on metal-plasma $\mathrm{L}$ band antenna.

The phase speed of electromagnetic waves in a plasma is given by:

$$
v_{p}=\frac{c}{\sqrt{1-\omega_{p}^{2} / \omega^{2}}}
$$

Where the plasma frequency is given by:

$$
\omega_{p}=\sqrt{\frac{n_{e} e^{2}}{\epsilon_{0} m_{e}}}
$$


In this paper, we are experimenting in the region where the antenna frequency is greater than the plasma frequency:

$$
\omega>\omega_{p}
$$

In this region refraction and not reflection takes place.

The phase speed of electromagnetic waves in a plasma is greater than in free space. The greater the density of the plasma the greater the phase speed. Since the plasma density can be reconfigured, the steering and focusing of antenna beams by the physics of refraction through a plasma is reconfigurable [10,11]. The amount of refraction through a plasma depends on the path length through a plasma and the change in plasma density over that path length [12]. This physical process can also be considered as a plasma lens [13].

Refraction in a plasma depends on:

1.Plasma density

2. Path length

\section{Gradient of plasma density}

We have very good results at $24 \mathrm{GHz}$ and above using COTS plasma tubes. We made custom plasma tubes with larger diameter and refraction that worked well at $10 \mathrm{GHz}$.

At $24 \mathrm{GHz}$, two plasma tubes were used to get antenna beam focusing and one plasma tube was used to get antenna beam spreading. Antenna beam steering was achieved with one and two plasma tubes at $24 \mathrm{GHz}$. In another case our beam steering experiments from using the physics of refraction through a plasma were done at $44 \mathrm{GHz}$.

\section{Focusing antenna beams with the physics of refraction through plasma}

In the following sections we show our work on antenna beam focusing, beam spreading, and beam steering using refraction of $\mathrm{RF}$ waves in a plasma. This is our first iteration of the plasma lens work and it can only improve. We found it was easier to show the lensing effects of plasma at $24 \mathrm{GHz}$ since the size and shape of COTS plasma tubes are amenable to a $24 \mathrm{GHz}$. These effects all scale according to wavelength, but cylindrical annular rings of plasma are the best way to control the plasma density variations of plasma to optimize the engineering effects of plasma refraction to control beam focusing, beam spreading, and beam steering.

We have demonstrated the ability to use a plasma for manipulation of a microwave signals by focusing a wide beam into a more narrow beam and also by steering the beam.

Figure 1 shows the experimental set-up for beam steering and lensing. A narrow-beam $24 \mathrm{GHz}$ signal is directed into the side of two 1.5 inch diameter plasma tubes which focuses the antenna beam into higher directivity, gain, and range.

This change in velocity of the signal inside the plasma results in a lensing effect if the beam passes through varying lengths of plasma similar to light passing through glass of varying thickness to make a lens. But there is an important and interesting difference between an ordinary lens made of glass or plastic and a plasma lens: The glass lens slows-down the signal while a plasma lens speeds-up the signal. Therefore 
a convex glass lens focuses a signal to a point while a convex plasma diverges the signal similar to a concave glass lens that diverges the signal while a concave plasma lens focuses to a point.

We have built converging and diverging plasma lenses using plasma tubes as shown in Figures 1 and 2. A single plasma tube with the beam passing through its diameter acts as a diverging plasma lens (Figures 2 and 3) and two plasma tubes side-by-side form a converging (focusing) lens (Figures 1 and 4).

We have built the "concave" set-up for beam shown in Figure 1, and Figure 4 is a $24 \mathrm{GHz}, 5 \mathrm{~mW}$ Gunn diode is used as the microwave source with the signal

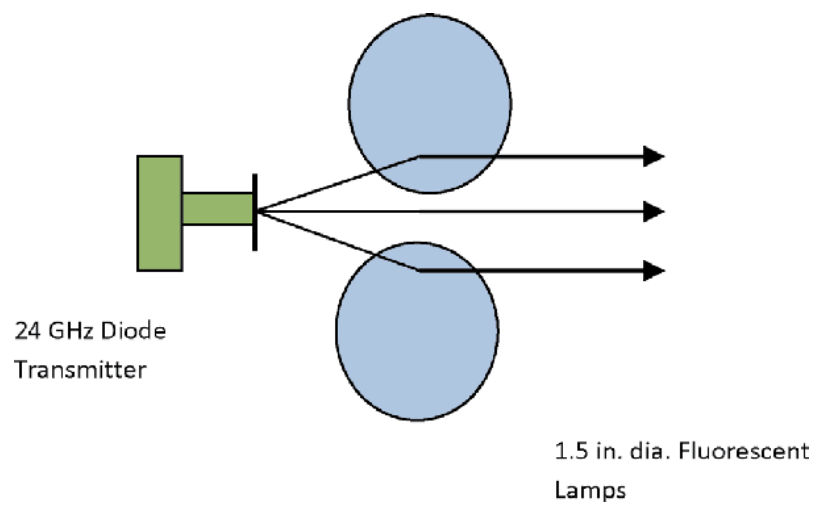

Figure 1.

Schematic for experimental setup of antenna beam focusing with tubes with plasma using two COTS (commercial off the shelf) tubes.

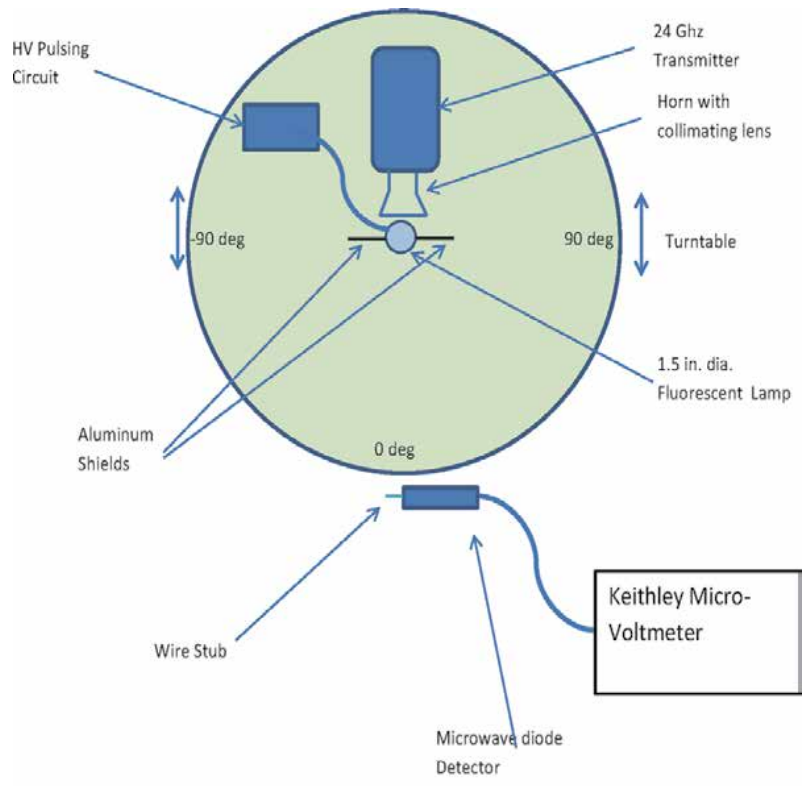

Set-up for measuring plasma lensing effect

Figure 2.

Schematic for experimental setup for antenna beam spreading with tubes with plasma in one COTS (commercial off the shelf) tubes. 


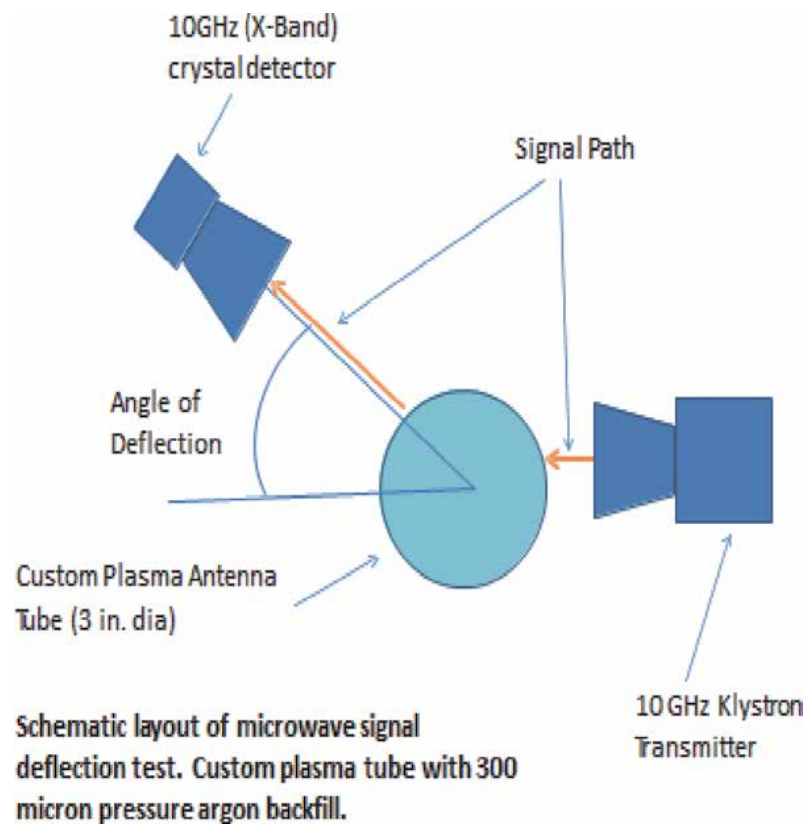

Figure 3 .

Schematic showing antenna beam spreading with one 3-inch diameter custom made plasma tube at $10 \mathrm{GHz}$.

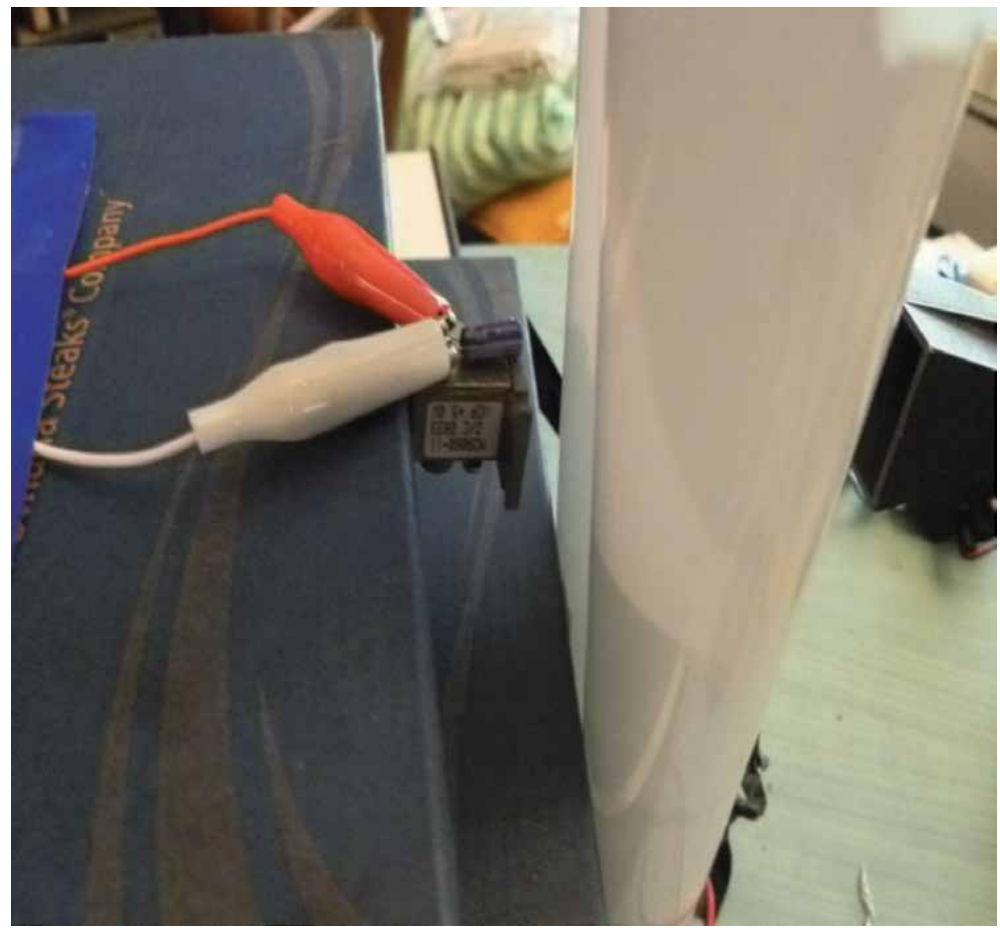

Figure 4.

Plasma focusing experimental setup from a different angle. Gunn diode $24 \mathrm{GHz}$ transmitter with fluorescent tubes used for plasma beam focus.

radiating from the open waveguide, which gives a directional but unfocused microwave output. This setup allows us to focus the beam in the forward direction resulting in a gain of $2(3 \mathrm{~dB})$. This can be seen in Figure 5 . 


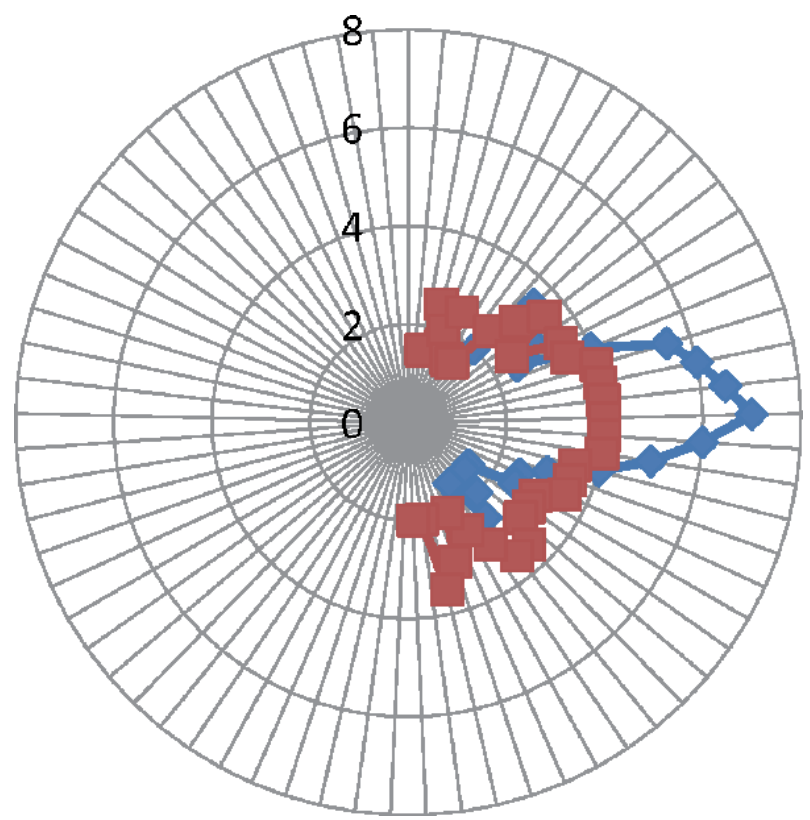

Figure 5 .

$24 \mathrm{GHz}$ beam focus with plasma. Red line is with no plasma. Blue line is with plasma. Note that the plasma focus increases beam amplitude by a factor of two compared to no plasma ( $\sim 3 \mathrm{~dB}$ gain). A crystal waveguide detector was used as a receiver. Amplitude numbers are relative voltage readings from the crystal detector.

We are the only group utilizing a pulsed high voltage power supply to give a much higher average plasma density and much lower average power to ionize the gas into plasma. This ability to tune the focusing of a RF beam is very useful because the same lensing structure can be used with different frequencies and to vary focal length as needed.

\section{Steering antenna beams with the physics of refraction through plasma}

We have done microwave beam steering using a cylindrical plasma tube. Those tests were done using fluorescents lamps with a diameter of 1.5 in $(3.8 \mathrm{~cm})$; and with a $24 \mathrm{GHz}$ microwave beam. A shortcoming of the set-up was that the $24 \mathrm{GHz}$ signal has a wavelength of $1.25 \mathrm{~cm}$, which is $1 / 3$ the diameter of the tube. For a properly working lens, the wavelength should be small compared to the physical dimensions of the lens, or in our case the plasma tube.

This work involved using a much higher frequency $(44 \mathrm{GHz}, 0.68 \mathrm{~cm})$ and therefore shorter wavelength. This wavelength is a factor of 5.6 smaller than the $3.8 \mathrm{~cm}$ tube diameter which should result in a more ideal lensing action. Our testing has confirmed this and has shown significantly narrower beam steering and less signal loss.

Of course all the dimensions scales with wavelengths used so going down to lower frequencies requires larger diameter tubes or other shaped plasma containers. This is why it is important to make custom made plasma tubes or other geometries.

Figure $\mathbf{6}$ is a schematic for the experimental setup, and Figures 7 and $\mathbf{8}$ are photos of the setup. A 33-50 GHz HP Microwave Signal Generator is used to generate the incident microwave beam. The plasma tube (fluorescent lamp) is placed $\sim 1$ inch in front of the open-ended waveguide, and an aluminum shield is placed against the side the tube to ensure that most of the microwave signal goes 


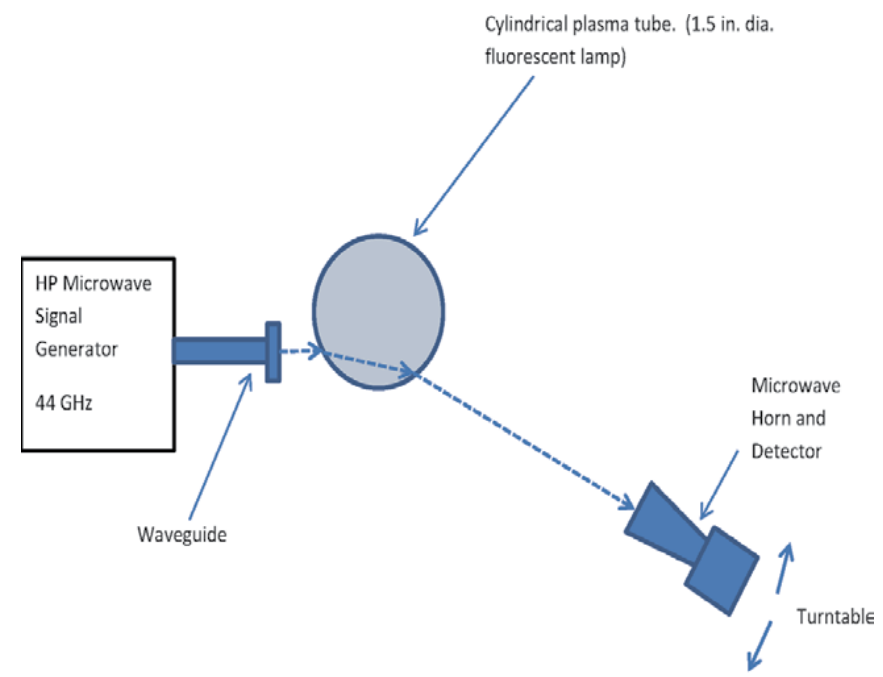

Schematic layout of $44 \mathrm{GHz}$ beam steering

experiment.

Figure 6.

Schematic for antenna beam steering using one COTS tube with plasma.

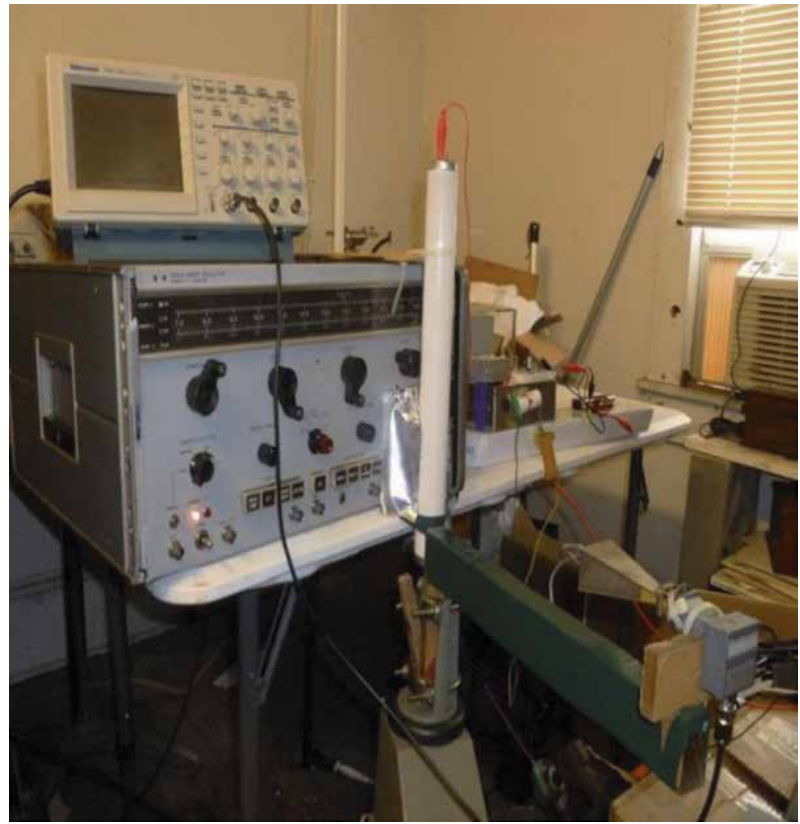

Figure 7.

Plasma beam steering experiment. Antenna rotator with holder (green) and receiver horn/detector is in the foreground. The oscilloscope used to monitor the signal waveform is on top of the HP signal generator. The solid state pulsing circuit is to the right of the signal generator.

through the plasma and is not bypassed to the side of the tube. A 33-50 GHz microwave horn with HP crystal detector is placed on a rotating arm that is scanned in an arc around the plasma tube by an antenna rotator. The arm places the horn 
receiver about 16 inches from the plasma tube. This corresponds to 60 wavelengths between the steering plasma tube and the detector and clearly qualifies as far field.

In order to generate a plasma with a density high enough to interact with $\mathrm{RF}$ signals in microwave frequencies, we use short current pulses $(\sim 1 \mu \mathrm{s})$ that quickly ionize the plasma; then rely on the plasma ions rather slowly migrating to the wall of the plasma tube. Using this technique (developed by Dr. Theodore Anderson and the late Professor Igor Alexeff) we generate a much higher average density plasma with a low average current and power.

Our high current pulses have so far had a period of $1 \mathrm{~ms}$. With this time separation between pulses, the plasma density decays by about a factor of two

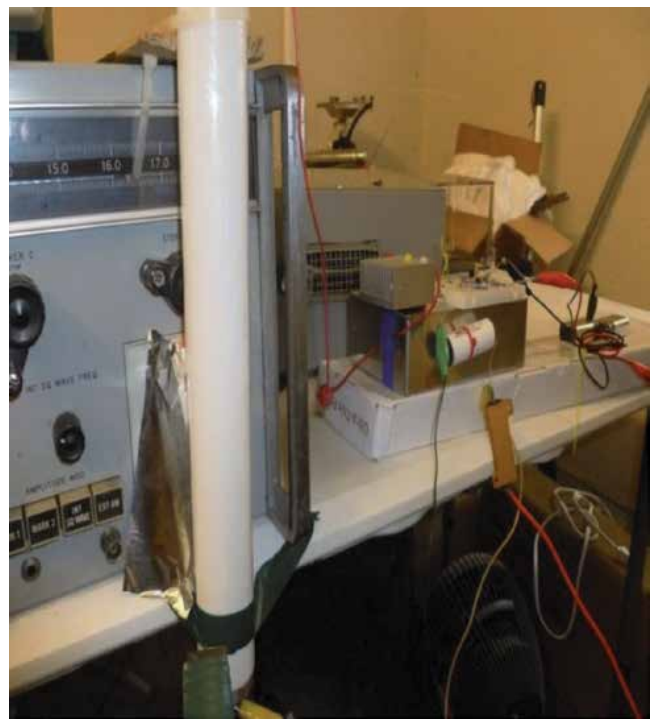

Figure 8.

Fluorescent plasma tube is located in front of the output waveguide on the HP signal generator. Aluminum shield on left of tube prevents stray RF from bypassing the plasma. High voltage pulser is on the right.

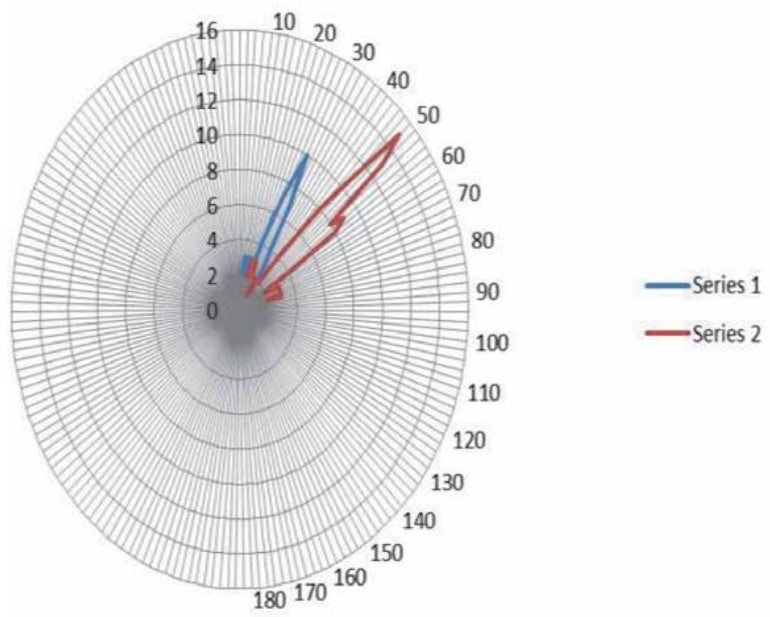

Figure 9.

Beam steering ( $44 \mathrm{GHz}$ ) for two different plasma ionizing currents. Blue line: 5 A peak. Red line: 8 A peak. A crystal waveguide detector is used as a receiver. Amplitude numbers are relative voltage readings from the crystal detector. 
before the next pulse comes to refresh the plasma back to peak density. This has not caused a problem with the smart antenna because we are using the plasma to totally block the RF signal, like a shutter. But lensing and beam steering require a plasma of a specific density to get a consistent beam deflection angle.

We are avoiding this problem by recording the received microwave signal immediately after the current pulse; therefore measurements are taken at a constant plasma density, which is also the maximum density before significant decay of density occurs.

Our recent experimental results with $44 \mathrm{GHz}$ show dramatic improvement in beam deflection characteristics compared to previous testing at $24 \mathrm{GHz}$. The shorter wavelength compared to tube dimensions has clearly resulted in cleaner and more consistent beam steering.

Figure 9 shows beam deflection at two peak ionization currents. The blue curve shows about 26 deflection with a current of $5 \mathrm{~A}$ peak. The red curve shows about 50 deflection with a current of 8 A peak.

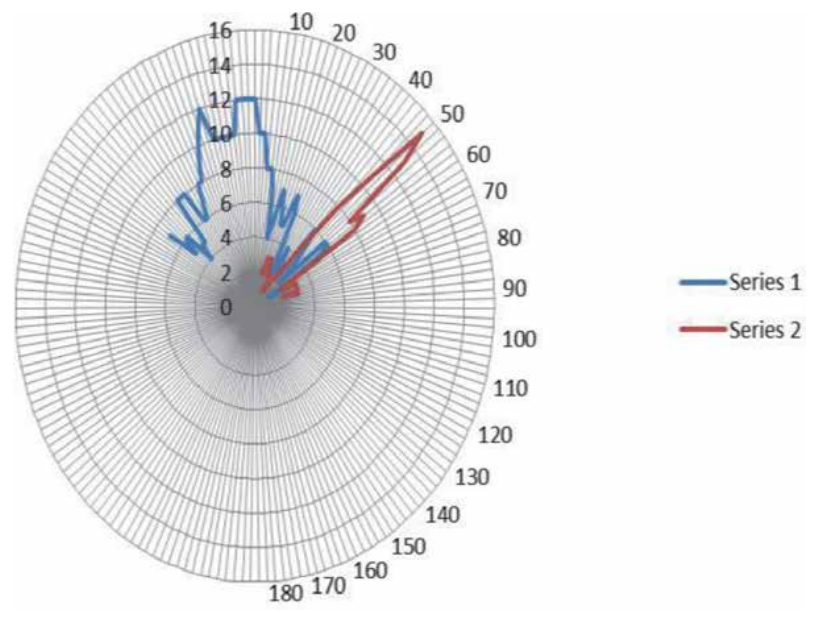

Figure 10.

Plasma beam steering. Beam is steered $\sim 45^{\circ}$ clockwise. Blue line: No plasma. Red line: 8 A peak ionizing current. A crystal waveguide detector is used as a receiver. Amplitude numbers are relative voltage readings from the crystal detector.

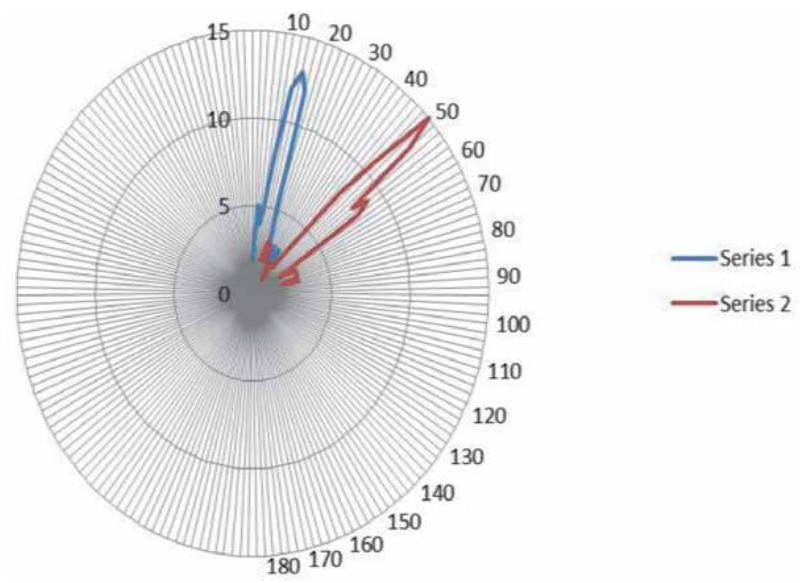

Figure 11.

Beam steering for two different plasma ionizing currents. Blue line: 3 A peak. Red line: 8 A peak. A crystal waveguide detector is used as a receiver. Amplitude numbers are relative voltage readings from the crystal detector. 
Figure 9 demonstrates that we can vary the angle of deflection by changing plasma ionizing current; but the most striking result shown in Figure 9 is the very narrow beam-width of the deflected signals. This is quite surprising. Figure 10 shows the 50 deflected beam (red line) along-side the un-deflected beam with no plasma. The incident un-deflected beam has a much wider beam-width compared to the deflected beam with plasma turned on.

Figure 11 shows the beam deflection (blue line) with a lower peak plasma current of $3 \mathrm{~A}$ with a beam deflection of $15^{\circ}$. For comparison the $50^{\circ}$ beam deflection with $8 \mathrm{~A}$ peak is shown in red.

\section{Pulsed plasma antenna circuitry}

A plasma antenna operating in the microwave frequency range requires higher operating currents (Greater than $\sim 1 \mathrm{~A}$ ) and consequently can overheat when used continuously. Our plasma antennas are able to work CW at high frequencies $(>1 \mathrm{GHz})$ because of a concept invented by Igor Alexeff and Theodore Anderson that uses fast high-current pulses; instead of DC current.

The plasma initiates quickly in less than a microsecond, but when plasma ionizing current is turned off, the ions take about a millisecond to recombine with electrons. Therefore plasma density stays high for almost a millisecond even though the ionizing current is no longer on. We use an even short pulse width $(\sim 1 \mu \mathrm{s})$, and therefore less power is required to run the antenna.

We developed a pulsed voltage doubler circuit, allowing us to use a lower voltage DC power supply for the input power to the pulsing circuit using a modified Marx Generator. A Marx Generator is a pulsed voltage multiplier. A series of capacitors was charged in parallel and then discharged in series through spark gaps.

Figure 12 shows a two stage voltage doubler circuit. We have built and tested a modified Marx Generator that replaces the spark gaps with an IGBT electronic switch.

Keeping the second spark gap in the circuit results in a faster rise time than in our previous pulsing circuits. The voltage doubler allows the use of a lower voltage DC power supply than would otherwise be required.

A simple non-voltage multiplying IGBT pulsing driver circuit is shown in Figure 13. A CMOS timer IC is used to generate short $1 \mu$ s pulses with a repetition time of $750 \mu \mathrm{s}$. An IXYS brand 2500 V IGBT is the high voltage switch. This pulsing arrangement allows us to use a factor of 750 less DC current and power from the DC power supply.

We have been driving our plasma antenna tube with the fast, high-current pulses described here to allow operation at higher frequencies than is possible with CW current. Our pulsing circuit had required that the both electrodes of the plasma tube operate at high voltage; with the positive electrode at constant maximum DC voltage $(2-3 \mathrm{kV})$. We have modified the circuit to allow the negative electrode to remain grounded while a positive $1 \mu$ s pulse is applied to the anode electrode. This change offers several advantages:

Improved electrical safety because one electrode remains grounded and the other electrode is supplied with short pulses. Less capacitive loss in the current leads allowing the use of faster pulses; since one electrode is grounded and can be attached to a ground plane.

Eliminating the need for a negative lead wire means less high voltage wiring. Lower EMI caused by high current pulses in wiring.

Grounding one side of the plasma tube requires that the high voltage switcher (IGBT) be able to float up and down in voltage with the tube's anode. To do this we 

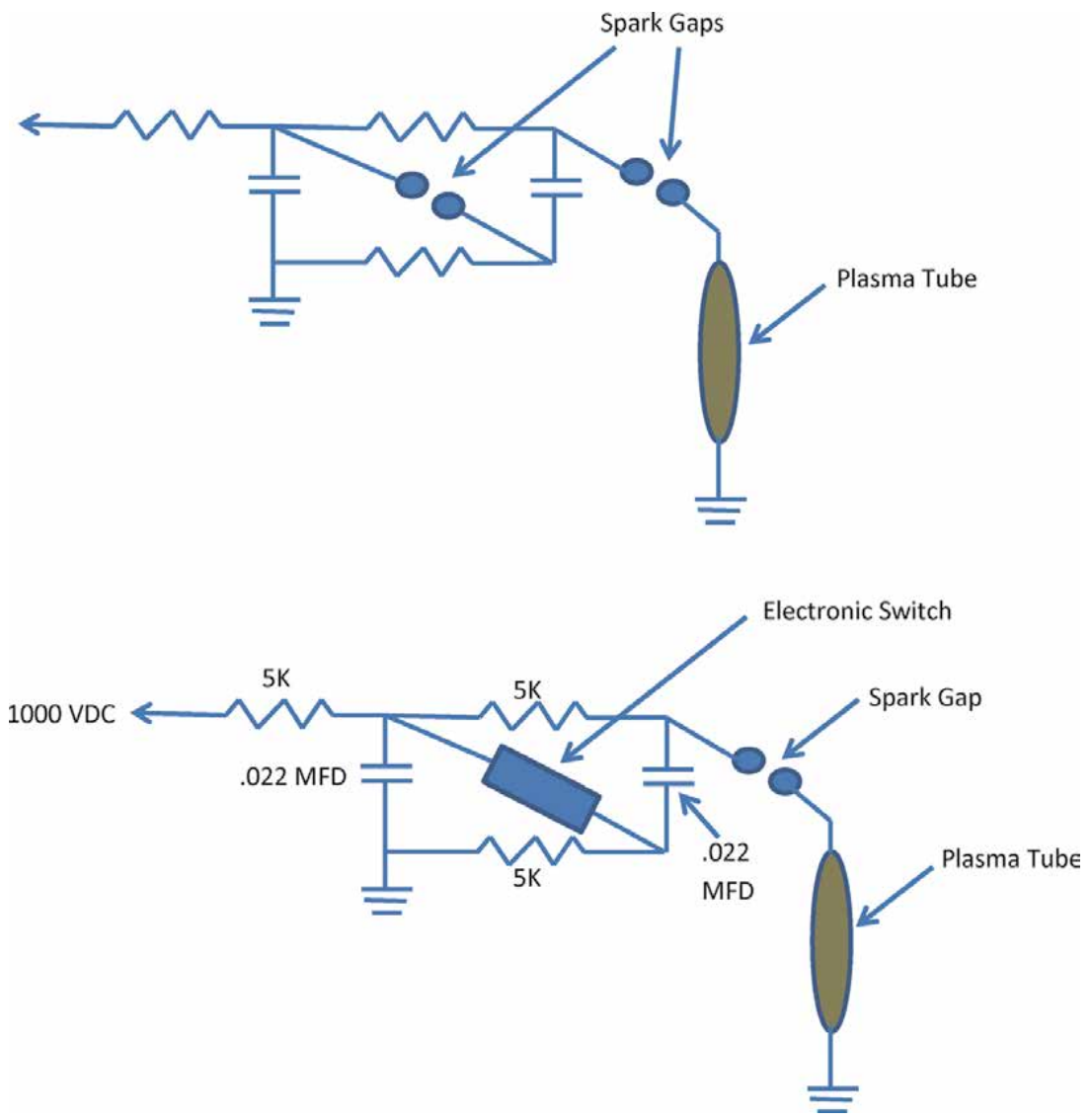

Figure 12.

$1000 \mathrm{VDC}$ to $2000 \mathrm{~V}$ pulse circuit using modified Marx generator with spark gaps in the top photo and with an electronic switch and spark gap in the bottom figure. Two electronic switches was also built but not shown.

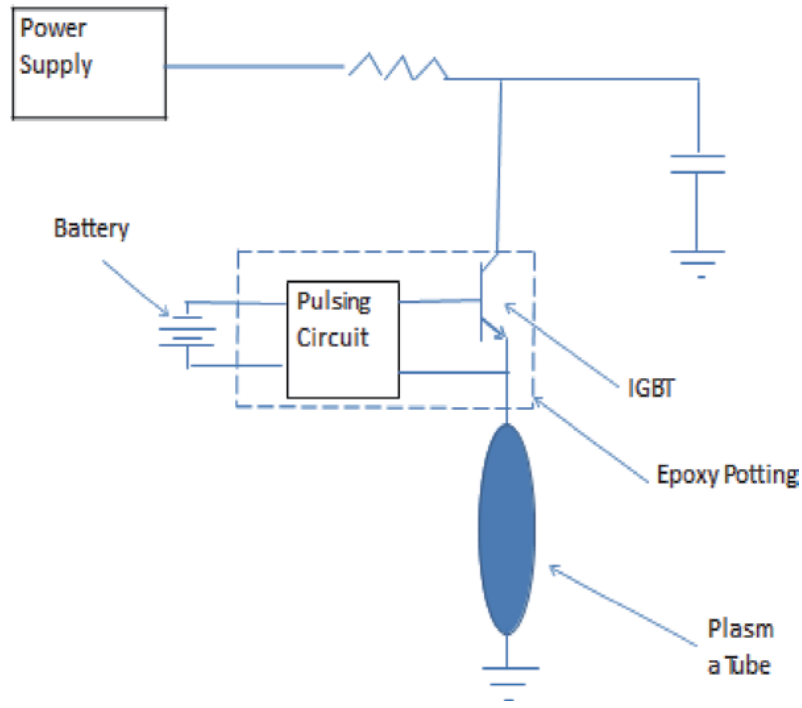

Figure 13.

Basic pulsing circuit. A DC power supply charges the capacitor, the IGBT pulsing circuit switching delivers short $\sim 1 \mu$ s pulses to the plasma tube. 


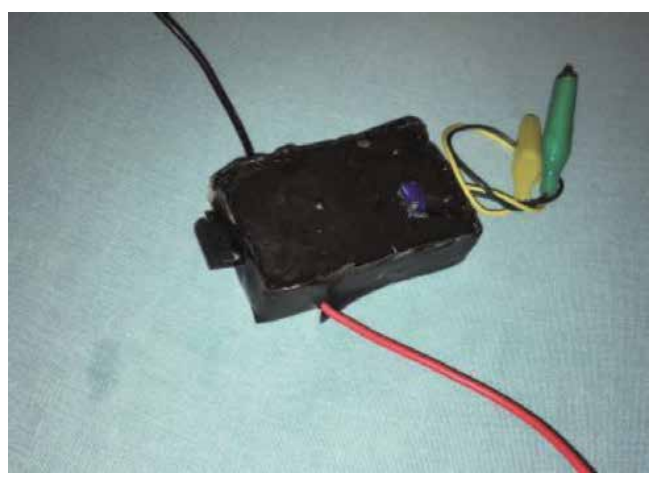

Figure 14.

Prototype pulsing circuit potted in epoxy. High voltage leads are at top and bottom, and the battery connection is on the right.

have electrically isolated the switcher by using a battery to power the electronics and by potting the IGBT and electronics in epoxy.

Figure 14 shows our first prototype with steel-filled epoxy; not the best choice of epoxy but one that is working quite well so far. Potting the circuit is advantageous for airplane and aerospace applications, providing mechanical ruggedness as well as electrical isolation. Epoxy has much higher thermal conductivity than air, but not as good as a metal heat sink. The steel filled epoxy has about a factor of 10 higher thermal conductivity than air. Highly thermally conductive epoxies can have a factor of 100 higher conductivity than air. Potting in epoxy in our case allowed operation without an additional metal heat sink, saving space and eliminating the need for electrical isolation between the IGBT and a metal heat sink. We tested the ruggedness of our epoxy-potted circuit by dropping it on a concrete floor from a height of $6 \mathrm{ft}$. without damage to the circuit.

We ran the circuit shown in Figure 13 (with no additional heat sink) with peak current of $20 \mathrm{~A}$ and a pulse period of $1000 \mu \mathrm{s}$.

After $1 / 2$ hour of operation, the epoxy and IGBT were warm to the touch but with no indication of over-heating.

\section{Power, current, and voltage requirements in pulsing excitations}

The plasma antenna requires a relatively high voltage, low current power supply.

Short pulses are applied to the terminals of the plasma tube. Peak current is about $1 \mathrm{~A}$ with $5 \mu$ s pulse width and a time between pulses of about $1 \mathrm{~ms}$.

This duty cycle of $1 / 200$ results in an average power of about $5 \mathrm{~W}$.

Overall average power drain from the battery driving the plasma antenna will be much less than $5 \mathrm{~W}$. Two standard $9 \mathrm{~V}$ batteries and/or one $6 \mathrm{~V} .75$ AH SLA battery can operate a plasma antenna. The smart plasma antenna can operate on a $12 \mathrm{~V}$ car battery which is enough voltage to ionize the plasma in 12 tubes and run the computer.

The power losses of the supply voltage connected to the plasma antenna are because the VSWR numbers in many cases indicate a very good match between the feeds and antennas.

Anyone trying to build a plasma antenna according should consult a licensed electrical safety expert before proceeding. After consulting a licensed electrical safety expert, proceed as follows. Use a three-wire grounded power cord and 
securely attach the green ground wire to the metal enclosure. Install an appropriately sized fuse or circuit breaker to protect from short circuits or overloads. Always unplug the unit before modifying or working inside.

\section{Conclusions}

We have demonstrated that one or two plasma tubes can be used to focus, spread, and steer antenna beams. We have also shown that we can simulate convex and concave plasma lenses by using cylindrical plasma tubes. Focusing by a plasma is useful because it can be used to increase the gain of an antenna, and to quickly reconfigure the beamwidth as needed without physically moving the antenna. With this technology, there is no need for phased arrays. Beam steering with a plasma allows tuning to different frequencies which is a difficult task for standard antennas. Our experimental results with $44 \mathrm{GHz}$ showed a dramatic improvement in beam steering and focusing characteristics compared to beam focusing and steering at $24 \mathrm{GHz}$. The shorter wavelength compared to the spatial variation in plasma density over the radius of the plasma tube, the easier it is to steer and focus antenna beams. These results have been incorporated in a new smart plasma antenna design which appears in another paper.

Driving the plasma with short high-current pulses allows CW operation at higher frequencies with a minimum amount of ionization power and higher plasma densities. Circuits for pulse forming and voltage multiplication are presented. The maximum frequency that a plasma antenna can operate $\mathrm{CW}$ has previously been limited by the high DC current needed to ionize the plasma. We minimize the average ionization power and increase the plasma density by using fast current pulsing with a short duty cycle. The average current is much lower but the average plasma density remains high than in the DC mode.

\section{Author details}

Theodore Anderson

Haleakala R\&D, Inc., Brookfield, Massachusetts, USA

*Address all correspondence to: tedanderson@haleakala-research.com

\section{IntechOpen}

(C) 2020 The Author(s). Licensee IntechOpen. This chapter is distributed under the terms of the Creative Commons Attribution License (http://creativecommons.org/licenses/ by/3.0), which permits unrestricted use, distribution, and reproduction in any medium, provided the original work is properly cited. (c) BY 


\section{References}

[1] Zhou J, Fang J, Lu Q, Liu F. Research on radiation characteristic of plasma antenna through FDTD method. Scientific World Journal. 2014;2014: 290148. DOI: $10.1155 / 2014 / 290148$

[2] Bogachev NN, Gusein-Zade NG, Nefedov VI. Radiation pattern and radiation Spectrum of the plasma asymmetrical dipole antenna. Plasma Physics Reports. 2019;45(4):372-375

[3] Kiss'ovski Z, Vachkov V. Model of a miniature plasma antenna. International Journal of Engineering and Advanced Technology (IJEAT). 2015;4(6): 2249-8958

[4] Golazari SS, Amiri N, Kashani FH. Design, simulation andmeasurement of loop plasma antenna in UHF band. In: 24th Telecommunications Forum (TELFOR). Belgrad; 2016

[5] Barro OA. Mohammed Himdi, and Olivier Lafond, reconfigurable cylindrical plasma antenna. Progress In Electromagnetics Research (PIER) M. 2018;66:65-72

[6] Kumar R, Kumar P. Study of array plasma antenna parameters. AIP Advances. 2018;8:045306. DOI: $10.1063 / 1.5018660$

[7] Melazzi D, Lancellotti V, De Carlo P, Manente M, Pavarin D, Anderson T. Numerical investigation into the performance of two reconfigurable gaseous plasma antennas. EuCAP 2014 Symposium Journal; 2014

[8] Anderson T, Melazzi D, Lancellotti V. An overview of experimental and numerical results on plasma antenna arrays. EuCAP 2015 Symposium Journal; 2015

[9] Giulia M, Paolo R, Mohammad Abdul H, Federico B, Capobianco AD, De Carlo P, et al. Design of a reconfigurable metal-plasma L-band transmit-array antenna. IEEE International Symposium on Antennas and Propagation and USNC-URSI Radio Science Meeting - AP-S/URSI 2019 Symposium Journal; 2019

[10] Anderson T. Plasma Antennas. Artech House. 2011:187-193

[11] Anderson T. Plasma Antennas. Artech House. 2011:177-185

[12] Anderson T. Plasma devices for steering and focusing antenna beams. U.S. Patent Issue Number: 8,384,602

[13] Linardakis P, Borg G, Martin N. Plasma-based lens for microwave beam steering. Electronics Letters. 2006; 42(8):444-446 

Section 5

Plasma Diagnostic Methods 



\title{
Plasma Diagnostic Methods: Test Charge Response in Lorentzian Dusty Plasmas
}

\author{
Shahid Ali and Yas Al-Hadeethi
}

\begin{abstract}
Different plasma diagnostic methods are briefly discussed, and the framework of a test charge technique is effectively used as diagnostic tool for investigating interaction potentials in Lorentzian plasma, whose constituents are the superthermal electrons and ions with negatively charged dust grains. Applying the space-time Fourier transformations to the linearized coupled Vlasov-Poisson equations, a test charge potential is derived with a modified response function due to energetic ions and electrons. For a test charge moving much slower than the dustthermal speed, there appears a short-range Debye-Hückel (DH) potential decaying exponentially with distance and a long-range far-field (FF) potential as the inverse cube of the distance from test charge. The FF potentials exhibit more localized shielding curves for low-Kappas, and smaller effective shielding length is observed in dusty plasma compared to electron-ion plasma. However, a wakefield (WF) potential is formed behind the test charge when it resonates with dust-acoustic oscillations, whereas a fast moving test charge leads to the Coulomb potential having no shielding around. It is revealed that superthermality and plasma parameters significantly alter the DH, FF, and WF potentials in space plasmas of Saturn's E-ring, where power-law distributions can be used for energetic electrons and ions in contrast to Maxwellian dust grains.
\end{abstract}

Keywords: kinetic model, test charge technique, DA waves, superthermal tails, dynamical shielding

\section{Plasma diagnostic methods}

To understand a plasma state and its characteristics, numerous experimental techniques, mechanisms, devices, theoretical models, and computational packages have been developed as diagnostic tools for measuring the plasma parameters such as the plasma electron density and temperature $[1,2]$ as well as their spatial profiles and dynamics. These diagnostic techniques are used to adequately describe both low-temperature and high energy density plasmas. In some situations, the measurements by these techniques cause perturbations in plasmas and are termed as active diagnostic techniques, while passive ones do not perturb plasmas. Based on the degree of ionization, the plasmas can broadly be classified into cold and hot plasma states, which accordingly demand for various types of diagnostics to 
precisely estimate the plasma parameters for optimal understanding of the physics of plasmas. This includes both theoretical and experimental findings. The most common techniques for cold and hot plasmas $\left(T_{e} \geq\right.$ few $\left.\mathrm{keV}\right)$ are the Langmuir probes in the form of planar, cylindrical, or spherical electrodes in plasmas with a goal to monitor the plasma parameters. The probes can be of several types, namely, single and/or double probes, which are used for density, temperature, and floating potential measurements. Emissive and magnetic probes work more efficiently for plasma potential measurement and wave field amplitude and phase diagnostics, respectively, while the Rogowski for antenna current measurements. There are many complications, for example, the plasma potential and density can fluctuate or drift during the time of probe measurements. In some complications, since the probe draws a large amount of currents from a plasma and perturbs the initial state of the plasma, it may lead to the erroneous measurements; even then, the Langmuir probe diagnostics are widely used and this is because of the fact that they are relatively simple to use, cheap, and give reliable values of important plasma parameters.

On the other hand, in certain plasma sources like tokamak plasmas, strong currents are generated, which give rise to various kinds of magnetohydrodynamic (MHD) instabilities. For this, a magnetic probe is used, which is beneficial especially for measuring either local magnetic fields or its fluctuations not only in tokamaks but also in laser-produced plasmas (LPP). Furthermore, the amplitude of current flowing into the plasma can be estimated by integrating the induced magnetic field around the plasma column by utilizing the so-called Rogowski coil. However, in some plasmas (especially high temperature), it is not feasible to utilize material probes for determining the plasma parameter like plasma electron density. Therefore, a nonperturbing approach is needed to diagnose the plasma. In such a scenario, the electromagnetic spectrum is utilized. But the electromagnetic wave intensity must be low enough to the level that it will not result in plasma perturbation. For probing the high-density plasmas, a lower wavelength is required as a probe. This justifies the utilization of infrared radiation in tokamak and ultraviolet radiation for measuring the plasma electron density in LPP. The variation of the polarizing angle involving the beam probe in the presence of magnetic field can also be used for diagnostics of tokamak plasmas.

Interestingly, the evaluation of appropriate plasma parameters may be carried out by spectroscopy of emitted radiations as used generally from the beginning of plasma physics. This technique for emission measurements has been particularly making significant contributions over the past five decades for the fact that plasmas produced for nuclear fusion research exhibit intense emission in the X-ray region. Astrophysical applications further justify the wide interest in X-ray emission from plasmas. The phase soft X-ray (so defined due to their low penetrating power) indicates electromagnetic radiations with a wavelength in range $1 \AA \leq \lambda \leq 300 \AA$ (or, in terms of photon energy $h v, 300 \mathrm{eV} \leq h \nu \leq 10 \mathrm{keV}$ ). Hard X-rays below $1 \AA$ are occasionally produced in plasmas for highly accelerated electrons, like runaway electrons in tokamak plasmas and suprathermal electrons in LPP [3]. The characteristics of soft X-ray spectra like line intensities, line profiles, and continuum intensities can be investigated to determine the electron densities by Stark broadening, while the ion densities from the absolute radiation intensities and ion temperatures using the Doppler broadening of spectral lines [2].

The particle measurement method is another scheme for investigating the characteristics of plasmas by using the beam of fast particles. It has received much attention in the studies of inertial confinement fusion and energy deposition in a medium driven by cluster-ion and fast heavy-ion beams, as well as in plasma accelerators and low-temperature laboratory plasmas. 


\section{Dusty plasma and test charge technique}

The most common ingredient of astrospace plasmas is the dust component in addition to electrons and ions, found everywhere in earth atmospheres, in comets, in planetary rings, in interstellar clouds, in interplanetary space, in interstellar medium, etc. Dust grains may exist in the form of ice particles, metallic and dielectric materials, and are highly charged species due to different charging processes. For instance, the absorption of ambient electrons and ions on dust grain surface may lead to the negatively charged dust grains, while thermionic and secondary electron emissions as well as ultraviolet photoionization give rise to positively charged dust grains. Thus, a multispecies dusty plasma can be assumed as more complex plasma than conventional electron-ion plasma, for dust size, mass, and charge variations. Being an abundant component of the space and industrial plasmas [4, 5], dusty plasma has always attracted lots of interests for studying new distinct features of plasma modes $[6,7]$ with a static and dynamic background of dust grains both analytically and experimentally [8-10]. Numerous linear and nonlinear [viz., solitons, shocks, vortices, etc.] dusty modes and associated instabilities are investigated using the frameworks of perturbative and nonperturbative schemes.

The behavior of charged particles in plasmas can be described by the wellknown fluid and kinetic theories [4, 11], essentially helpful for studying the basic properties of plasma waves and instabilities, depending strongly on the observed phenomena. Laboratory plasmas have effectively been modeled by fluid description, where charged fluids of plasma species are assumed in temporal and spatial configurations. But, it has been observed that fluid theory does not account for velocity space coordinate and is insufficient to study the wave phenomena in nonequilibrium plasmas, where particle distributions show significant deviations from the equilibrium states. Hence, fluid theory is unable to explain the wave-particle interactions that could lead to collisionless Landau damping phenomenon and many other interesting features of collective modes and instabilities. Conversely, kinetic theory adequately describes the physical phenomena in real time and phase space configurations, providing all information about plasma waves, instabilities, plasma equilibrium, Landau damping rate, etc.

Test charge techniques $[12,13]$ can be utilized to study the shielding of test charges in collisional [14] and turbulent [15] plasmas, the electric field [16], and farfield potential of a test charge in a nonuniform magnetoplasma [17], the wake-field excitations in charge fluctuating dusty plasmas [18], the two-body correlations [19], the energy loss of test charges [20], etc. If a test particle is projected into the plasma with a constant speed, its charge density is coupled with the plasma charge density by the space charge effects. Consequently, the test charge is screened by a cloud of opposite sign charges leading to the short-range Debye-Hückel (DH) potential. Of course, the speed of test charge significantly matters in plasmas when it is considered with respect to thermal speeds of plasma species. The interaction potentials and energy loss of charged particles have been recognized in many research areas, for example, in ion-cluster interaction with condensed matter [21, 22], in inertial confinement fusion [23-25], in particle acceleration [26], in low-temperature laboratory plasmas [27, 28], and in dense plasmas for heavy-ion energy deposition [29].

Montgomery et al. [13] employed the test charge technique to obtain far-field potential distribution around a test charge, which decays as the inverse cube of the distance from test charge in electron-ion Maxwellian plasmas. Subsequent investigations of shielded potentials have been phenomenally influenced by the ionic motion [30], electron-electron collisions [14, 31], and plasma turbulence [15]. The electrostatic potential [32] due to small and large test charge velocities has been 
investigated to display the excitation of long-range wakefields in Maxwellian plasmas. Shivamoggi and Mulser [33] examined the effects of magnetic field, collisions, and plasma inhomogeneity on the potential due to slowly and rapidly moving test charges [17, 34], and Lakshmi et al. [35] discussed the Debye shielding phenomenon in a dusty plasma by considering the Boltzmannian electrons and ions with cold negative dust grains. It was revealed that plasma parameters significantly alter the characteristics of small and large amplitude potentials. Later, Shukla [36] reported the FF potential for a slowly moving test charge in a Maxwellian dusty plasma and showed the impact of dust-charge variation on the dipole-like FF potential. Moreover, oscillatory wake-field can be excited behind the test charge [37] in a collisionless unmagnetized plasma with Maxwellian electrons and ions. Nambu et al. [38] extended this work to dusty plasmas and explained the resonant phenomenon of DA waves with a test charge, resulting in the long-range WF potential. Later, Shukla and Rao [39] analyzed the WF, DH, and FF potentials of test charge in a colloidal Maxwellian plasma accounting for the streaming ions and dust grains. It was found that external magnetic field and ion-streaming effects $[40,41]$ strongly affect the positive/negative potential regions in plasmas. To explore the effects of two-body correlations, dust-charge perturbations and dustneutral collisions, various geometries have been designed for propagating test charges [18-20] in an unmagnetized Maxwellian dusty plasma. In all above investigations, the plasma particles are described by the Maxwellian distribution function.

The shielding phenomenon is one of the main objectives of this chapter to unfold many intrinsic properties of the Lorentzian space dusty plasma, which discerns it from the standard Maxwellian plasmas. It plays a key role in setting up the basic criteria for Lorentzian dusty plasmas. Any plasma medium can physically be polarized by the test charge to give rise to perfect screening if thermal agitations are absent in the plasma system. Conversely, an imperfect shielding occurs if the plasma particles get enough thermal energy to escape from the edge of screening cloud. The interaction potentials caused by the test charge are not only strongly influenced by different test charge speeds in comparison with the thermal speeds but also lead to the possibility of dust crystallization and dust coagulation in space Lorentzian dusty plasmas.

\section{Power-law Lorentzian distribution function (df)}

In some circumstances, the behavior of plasma particles cannot be described by the usual Maxwellian distribution function (df) but often modeled by the powerlaw df. When all or some of the plasma particles move faster than their thermal speeds, the plasma particles are known as superthermal/suprathermal species, showing high energy and velocity tails in the distribution. They are mostly accelerated by wave-particle interactions, modulational instabilities and Langmuir turbulence [42], beam-plasma interactions [43], solar wind where type III solar radio emissions occurs [44], intense microwave-plasma interactions [45], ionospheric heating experiments [46], etc. Recognizing the role of superthermal energetic particles in plasmas, the wave dynamics and instabilities need to be re-investigated with a power-law $\mathrm{df}$ that gives a better fit to empirical data from space plasmas.

A 3D isotropic Kappa-df [47] for superthermal particles can be expressed as

$$
f_{s 0}^{K}\left(\mathbf{v}_{s}\right)=A_{\kappa_{s}}\left(1+\frac{v_{s}^{2}}{\kappa_{s} \theta_{T s}^{2}}\right)^{-\kappa_{s}-1}
$$



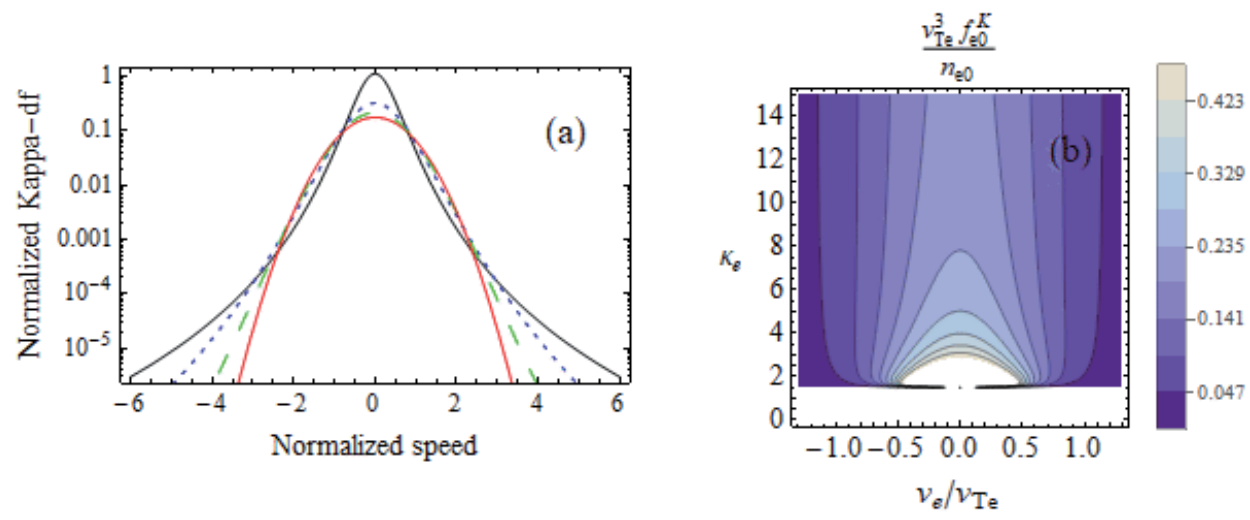

Figure 1.

Kappa-df against the electron speed for different values of kappa index both in $2 D$ and in contour plots. In (a), the solid-black, dotted-blue, dashed-green, and solid-red curves correspond to $\kappa_{e}=2,4,10$, and $\kappa_{e} \rightarrow \infty$, respectively. However, in $(b)$, the light colors indicate more number of electrons and vice versa for dense colors.

The normalization constant and effective thermal speed are denoted by $A_{\kappa_{s}}=$ $n_{s 0} \pi^{-3 / 2} \kappa_{s}^{-3 / 2} \theta_{T s}^{-3} \Gamma\left(\kappa_{s}+1\right) / \Gamma\left(\kappa_{s}-1 / 2\right)$ and $\theta_{T s}=\left\{2\left(\kappa_{s}-3 / 2\right) / \kappa_{s}\right\}^{1 / 2}\left(T_{s} / m_{s}\right)^{1 / 2}$, respectively. The symbol $\Gamma$ indicates the Gamma function, and $\mathbf{v}_{s}$ and $n_{s 0}$ are the velocity and equilibrium number density, whereas $T_{s}\left(m_{s}\right)$ stands for the temperature (mass) of the $s t h$ species ( $s=e$ for electrons and $i$ for positive ions). The effective thermal speed $\theta_{T s}$ is always realistic in the limit $\kappa_{s}>3 / 2$, where $\kappa_{s}$ is the spectral index showing the deviation from the Maxwellian df. Figure 1 displays the normalized Kappa-df [as given by Eq. (1)] for the electrons as function of normalized electron speed with varying $\kappa_{e}$-index both in 2D and contour plots. See that superthermal electrons exhibit high energy tails at $\kappa_{e}=2,4$, and 10 in distribution curves, which tend to the Maxwell-Boltzmann distribution curve for $\kappa_{e} \rightarrow \infty$.

Therefore superthermality effects are only significant for low values of Kappa, and for its infinite values, the Kappa-df exactly converges to the Maxwell-df $\left[\right.$ viz., $\left.f_{s 0}^{K}\left(\mathbf{v}_{s}\right) \rightarrow f_{s 0}^{M}\left(\mathbf{v}_{s}\right)\right]$. It may be noted from contours (see Figure $\mathbf{1}(\mathbf{b})$ ) that light-colored regions correspond to more electrons at low speeds, and while moving toward the dense-colored regions, the number of electrons decreases but comparatively has high speeds. In 1968, for the first time, Vasyliunas [47] pointed out the implications of Kappa-df by fitting empirical data from solar wind and showed the significance of low values of electron spectral index, that is, $\kappa_{s=e} \sim 2-4$. The effects of high energy tails have significantly modified the dispersive properties of waves and instabilities [48, 49] in Lorentzian plasmas. Recently, Ali and Eliasson [50] investigated the impact of suprathermal hot electrons on the electrostatic potential of slowly moving test charge in a two-temperature electron plasma and extended the model for Lorentzian dusty plasmas [51].

\section{Kinetic model for Lorentzian dusty plasmas}

To compute the potential distributions around a test charge, we consider a collisionless Lorentzian dusty plasma, containing the suprathermal electrons and ions with negatively charged dust grains following the Maxwell-df. The plasma is also assumed to be field-free in the sense that there is no external electric or magnetic field (viz., $E_{0}=0=B_{0}$ ), so that the equilibrium electrostatic potential $\phi_{0}=0$. The quasi-neutrality condition at equilibrium demands $n_{e 0}=n_{i 0}-Z_{d 0} n_{d 0}$, where $Z_{d 0}$ 
being the equilibrium dust-charge state and $n_{j 0}$ denote the particle number densities of the $j$ th species [ $j$ equals $s(=e, i)$ for Kappa-distributed electrons and ions while $j=d$ for negatively charged dust grains]. In this model, all the dust grains are assumed to be spherical in shape with constant size of radius $r_{d}$ and mass $m_{d}$.

The Lorentzian dusty plasma in the presence of a test charge can be described by the following linearized coupled set of Vlasov-Poisson equations:

$$
\begin{gathered}
\left(\partial_{t}+\mathbf{v}_{d} \cdot \nabla\right) f_{d 1}+\frac{q_{d 0}}{m_{d}} \mathbf{E}_{1} \cdot \nabla_{\mathbf{v}_{d}} f_{d 0}^{M}=0 \\
\left(\partial_{t}+\mathbf{v}_{s} \cdot \nabla\right) f_{s 1}+\frac{q_{s}}{m_{s}} \mathbf{E}_{1} \cdot \nabla_{\mathbf{v}_{s}} f_{s 0}^{K}=0
\end{gathered}
$$

and

$$
\nabla^{2} \phi_{1}+4 \pi\left(\sum_{s=e, i} \rho_{s}+\rho_{d}+\rho_{T}\right)=0
$$

where $\mathbf{E}_{1}\left(=-\nabla \phi_{1}\right)$ is the induced electric field with perturbed potential $\phi_{1}$, $q_{d 0}\left(=-Z_{d 0} e\right)$ is the charge of the negative dust grains, and $q_{s}(=-e, e)$ being the charge of electrons and positive ions. $\rho_{d}=q_{d 0} \int f_{d 1} d \mathbf{v}_{d}, \rho_{s}=q_{s} \int f_{s 1} d \mathbf{v}_{s}$, and $\rho_{T}=$ $q_{T} \delta\left(\mathbf{r}-\mathbf{v}_{T} t\right)$ identify the dust-charge density, electron-ion charge densities, and test charge density, respectively. The symbol $\delta$ stands for a 3D Dirac's delta function, and $f_{d 0}^{M}\left(\mathbf{v}_{d}\right)$ and $f_{s 0}^{K}\left(\mathbf{v}_{s}\right)$ are the Maxwell-df and Kappa-df with their perturbed parts $f_{d 1}\left(\mathbf{r}, \mathbf{v}_{d}, t\right)$ and $f_{s 1}\left(\mathbf{r}, \mathbf{v}_{s}, t\right)$, such that $\left|f_{d 1}\right| \ll f_{d 0}^{M}$ and $\left|f_{s 1}\right| \ll f_{s 0}^{K}$. Also note that test particle has a charge $q_{T}$ which moves with a constant velocity $\mathbf{v}_{T}$ along the z-axis in a Lorentzian dusty plasma.

Taking space-time Fourier analysis of Eqs. (2), (3), and (4), we obtain the Fourier transformed potential in this form

$$
k^{2} D(\mathbf{k}, \omega) \phi_{1}(\mathbf{k}, \omega)=8 \pi^{2} q_{T} \delta\left(\omega-\mathbf{k} \cdot \mathbf{v}_{T}\right) .
$$

The modified longitudinal dielectric constant can be defined by

$$
D(\mathbf{k}, \omega)=1+\sum_{s} \frac{\omega_{p s}^{2}}{k^{2}} \int \frac{\mathbf{k} \cdot \nabla_{\mathbf{v}_{\mathbf{s}}} f_{s 0}^{K}\left(\mathbf{v}_{s}\right)}{\left(\omega-\mathbf{k} \cdot \mathbf{v}_{s}\right)} d \mathbf{v}_{s}+\frac{\omega_{p d}^{2}}{k^{2}} \int \frac{\mathbf{k} \cdot \nabla_{\mathbf{v}_{\mathrm{d}}} f_{d 0}^{M}\left(\mathbf{v}_{d}\right)}{\left(\omega-\mathbf{k} \cdot \mathbf{v}_{d}\right)} d \mathbf{v}_{d},
$$

where $\omega(k)$ being the angular frequency (wave number) and $\omega_{p j}=$ $\left(4 \pi q_{j}^{2} n_{j 0} / m_{j}\right)^{1 / 2}$ is the plasma oscillation frequency. It is important to mention that if Lorentzian dusty plasma does not contain any test charge, viz., $q_{T}=0$, then Eq. (5) simply implies that $D(\mathbf{k}, \omega)=0$, showing a modified linear dispersion relation of electrostatic waves to account for superthermal electrons and ions. However, the inverse Fourier analysis of Eq. (5) leads to the standard form of electrostatic potential due to a test charge in a dusty plasma [11, 32].

$$
\phi_{1}(\mathbf{r}, t)=\frac{q_{T}}{2 \pi^{2}} \int \frac{d \mathbf{k}}{k^{2}} \frac{\exp \left[i \mathbf{k} \cdot\left(\mathbf{r}-\mathbf{v}_{T} t\right)\right]}{D\left(k, \mathbf{k} \cdot \mathbf{v}_{T}\right)} .
$$

The dielectric constant in terms of dielectric susceptibilities (viz., $D=1+\sum_{s=e, i} \chi_{s}+\chi_{d}$ ) can be expressed as 


$$
D\left(k, \mathbf{k} \cdot \mathbf{v}_{T}\right)=1+\sum_{s=e, i} \frac{1}{k^{2} \lambda_{D s}^{2}}\left\{1-\frac{1}{2 \kappa_{s}}+C_{s} Z_{\kappa_{s}}\left(C_{s}\right)\right\}+\frac{1}{k^{2} \lambda_{D d}^{2}}\left\{1+C_{d} Z_{M}\left(C_{d}\right)\right\}
$$

where $\lambda_{D s}\left(=\theta_{T s} / \sqrt{2} \omega_{p s}\right)$ and $\lambda_{D d}\left(=v_{T d} / \omega_{p d}\right)$ are the Debye shielding lengths associated with the electron-ion effective speed $\theta_{T s}$ and dust thermal speed $v_{T d}=$ $\left(T_{d} / m_{d}\right)^{1 / 2}$. The standard plasma dispersion functions for Kappa-distributed electrons and ions [48] and for Maxwellian dust grains [52], respectively, can be given by

$$
Z_{\kappa_{s}}\left(C_{s}\right)=\frac{1}{\sqrt{\pi}} \frac{\Gamma\left(\kappa_{s}+1\right)}{\kappa_{s}^{3 / 2} \Gamma\left(\kappa_{s}-1 / 2\right)} \int_{-\infty}^{\infty} d \beta_{s} \frac{\left(1+\beta_{s}^{2} / \kappa_{s}\right)^{-\kappa_{s}-1}}{\left(\beta_{s}-C_{s}\right)}, \quad \operatorname{Im}\left(C_{s}\right)>0,
$$

and

$$
Z_{M}\left(C_{d}\right)=\frac{1}{\sqrt{\pi}} \int_{-\infty}^{\infty} d \beta \frac{\exp \left(-\beta^{2}\right)}{\left(\beta-C_{d}\right)}, \quad \operatorname{Im}\left(C_{d}\right)>0
$$

with their corresponding arguments $C_{s}=\mathbf{k} \cdot \mathbf{v}_{T} / \sqrt{2}|k| \theta_{T s}$ and $C_{d}=\mathbf{k}$. $\mathbf{v}_{T} / \sqrt{2}|k| v_{T d}$.

To proceed further, we shall consider two limiting cases of Eq. (8) by imposing certain limitations on the test charge speed in comparison with the thermal and acoustic speeds and simplify the interaction potentials [as given by Eq. (7)] in Lorentzian dusty plasmas.

\subsection{Slow moving test charge response}

For a slow test charge propagation in a Lorentzian dusty plasma, we assume that test charge speed $\left(v_{T}\right)$ is much slower than the dust thermal speed $\left(v_{T d}\right)$. As a result, the test charge is shielded by all the plasma species, for example, electrons, ions, and dust grains. Since mass of the dust grains is larger than the mass of electrons and ions, therefore the dust thermal speed is much smaller than the electron and ion thermal speeds. Thus, imposing the inequalities $v_{T} \ll v_{T d} \lll \theta_{T s}$, we consider only small argument expansions, that is, $\left|C_{s}\right| \ll 1$ and $\left|C_{d}\right| \ll 1$, in the plasma dispersion functions to obtain a simplified expression for dielectric constant. The inverse of the latter eventually yields the following result

$$
D^{-1} \simeq \frac{k^{2} \lambda_{D \kappa}^{2}}{k^{2} \lambda_{D \kappa}^{2}+1}-i \frac{\mu v_{T}}{v_{T d}}\left(\frac{\pi}{2}\right)^{1 / 2} \frac{k^{2} \lambda_{D \kappa}^{4}}{\lambda_{D d}^{2}\left(k^{2} \lambda_{D \kappa}^{2}+1\right)^{2}}
$$

The modified effective Debye length $\lambda_{D \kappa}$ can be simplified in this form

$$
\lambda_{D \kappa}=\lambda_{0}\left(c_{\kappa e} \frac{n_{e 0}}{n_{0}}+c_{\kappa i} \frac{T_{e}}{T_{i}}+Z_{d 0}^{2} \frac{n_{d 0}}{n_{0}} \frac{T_{e}}{T_{d}}\right)^{-1 / 2},
$$

with superthermality parameters attributed to electrons and ions as

$$
c_{\kappa e}=\frac{2 \kappa_{e}-1}{2 \kappa_{e}-3} \quad \text { and } \quad c_{\kappa i}=\frac{2 \kappa_{i}-1}{2 \kappa_{i}-3} .
$$

The usual Debye length in electron-ion plasma is denoted by $\lambda_{0}=\left(T_{e} / 4 \pi n_{0} e^{2}\right)^{1 / 2}$ with $n_{0}=n_{i 0} \equiv n_{e 0}+Z_{d 0} n_{d 0}$ and $\mu\left(=\cos \theta_{k}\right)$ representing the angle between the 
vectors $\mathbf{k}$ and $\mathbf{v}_{T}$. For Maxwellian plasmas, the superthermality parameters $c_{\kappa e, i} \rightarrow 1$ as long as $\kappa_{e, i} \rightarrow \infty$, implying that the effective shielding length exactly coincides with the earlier result [35] for static cold dust grains. It may be noticed from Eq. (9) that the inverse of dielectric constant is significantly influenced by the dust Landau damping rate [13]. The importance of the latter was first pointed out [13] in 1968, and it was suggested that if a test charge moves slowly in comparison to the dust thermal speed, the dust Landau damping term appearing in Eq. (9) cannot be ignored. For a static test charge, we set $v_{T}=0$ and insert Eq. (9) into Eq. (7) to derive the short-range DH potential $[53,54]$ in the form $\phi_{D H}=\left(q_{T} /|r|\right) \exp \left(-|r| / \lambda_{D \kappa}\right)$, where $|r|=\left(\rho^{2}+\xi^{2}\right)^{1 / 2}$ is the distance from the test charge to an observer in terms of radial and axial distances $\rho$ and $(\xi=z)$, respectively.

For numerical analyses, we can choose the data from the dusty plasma near Saturn's E ring, cited in Refs. [55-58] and many references therein. The data essentially corresponds to the Radio and Plasma Wave Science (RPWS) instruments onboard the Cassini spacecraft, containing the plasma parameters, such as $n_{d 0}=0.1 \mathrm{~cm}^{-3}, n_{e 0}=70 \mathrm{~cm}^{-3}, Z_{d 0}=300, T_{d}=T_{e} / 10, T_{i}=T_{e} / 2$, and $T_{e}=4.642 \times 10^{5} \mathrm{~K}$. The computation further helps us in finding the magnitude of the effective shielding length $\lambda_{D \kappa}(=15.649 \mathrm{~cm})$ at the near-Maxwellian electrons and ions with $\kappa_{i, e}=100$. The impact of superthermal tails in the electron and ion distributions only appear at lower values of the Kappa that may result into the reduction of the shielding length $\lambda_{D \kappa} \approx 15.4787 \mathrm{~cm}$ for fixed $\kappa_{i}=1.6$ and $\kappa_{e}=100$, as well as $\lambda_{D \kappa} \approx 15.5888 \mathrm{~cm}$ with $\kappa_{e}=1.6$ and $\kappa_{i}=100$. Thus, we notice from Table 1 that for infinite values of the spectral indices, that is, $\kappa_{e, i} \rightarrow \infty$, the superthermality parameters $\left(c_{\kappa e, i}\right)$ tend to unity, implying that the effective shielding length exactly coincides with the previous results [35] for static cold dust grains. However, the variation due to superthermal electrons and ions is shown in three different combinations (see Table 1) to affect the normalized values of the effective Debye length and DH potential almost 4 digits beyond the decimal point. At lower Kappa-values, the impact is relatively enhanced and in turn, suprathermal ions more efficiently modify the effective shielding length and $\mathrm{DH}$ potential as compared to suprathermal electrons because the ions may take

\begin{tabular}{|c|c|c|c|c|c|c|}
\hline s\# & $\begin{array}{c}\text { Spectral-index } \\
\text { for electrons } \\
\kappa_{e}\end{array}$ & $\begin{array}{l}\text { Spectral-index } \\
\text { for ions } \\
\kappa_{i}\end{array}$ & $\begin{array}{c}\text { Electron-superthermality } \\
\text { parameter } c_{\kappa_{0}}\end{array}$ & $\begin{array}{l}\text { lon-superthermality } \\
\text { parameter } c_{\mathrm{K}_{\mathrm{i}}}\end{array}$ & $\begin{array}{l}\text { Effective Debye length } \\
\qquad \frac{\lambda_{D K}}{\lambda_{0}}\end{array}$ & $\begin{array}{l}\text { DH Potential } \\
\frac{\phi_{\mathrm{DH}}}{\left(\mathrm{q}_{\mathrm{T}} / \lambda_{0}\right)}\end{array}$ \\
\hline 1 & 100 & 100 & 1.01015 & 1.01015 & 0.0332829 & 0.0122817 \\
\hline 2 & 6 & 100 & 1.22222 & 1.01015 & 0.0332802 & 0.0122756 \\
\hline 3 & 4 & 100 & 1.4 & 1.01015 & 0.0332779 & 0.0122705 \\
\hline 4 & 3 & 100 & 1.66667 & 1.01015 & 0.0332745 & 0.0122629 \\
\hline 5 & 2 & 100 & 3 & 1.01015 & 0.0332573 & 0.0122249 \\
\hline 6 & 1.6 & 100 & 11 & 1.01015 & 0.0331548 & 0.0119997 \\
\hline 7 & 100 & 100 & 1.01015 & 1.01015 & 0.0332829 & 0.0122817 \\
\hline 8 & 100 & 6 & 1.01015 & 1.222222 & 0.03322751 & 0.0122644 \\
\hline 9 & 100 & 4 & 1.01015 & 1.4 & 0.0332686 & 0.0122499 \\
\hline 10 & 100 & 3 & 1.01015 & 1.66667 & 0.0332588 & 0.0122281 \\
\hline 11 & 100 & 2 & 1.01015 & 3 & 0.0332098 & 0.0121203 \\
\hline 12 & 100 & 1.6 & 1.01015 & 11 & 0.0329206 & 0.0114957 \\
\hline 13 & 100 & 100 & 1.01015 & 1.01015 & 0.0332829 & 0.0122817 \\
\hline 14 & 6 & 6 & 1.22222 & 1.22222 & 0.0332724 & 0.0122583 \\
\hline 15 & 4 & 4 & 1.4 & 1.4 & 0.0332636 & 0.0122387 \\
\hline 16 & 3 & 3 & 1.66667 & 1.66667 & 0.0332503 & 0.0122095 \\
\hline 17 & 2 & 2 & 3 & 3 & 0.0331823 & $0.01206 n 3$ \\
\hline 18 & 1.6 & 1.6 & 11 & 11 & 0.0327966 & 0.0112346 \\
\hline
\end{tabular}

Table 1.

The electron and ion Kappa-indices affect the values of the effective Debye length and DH potential at fixed values of $r=0.2, \eta=10^{-3}$, and $Z_{d o}=300$. 
more time to attain the Maxwellian equilibrium due to their larger mass compared to electrons.

The effect of dust concentration [denoted through the parameter $\eta\left(=n_{d 0} / n_{0}\right)$ ] is shown on the contours of normalized effective shielding length [as given by Eq. (10)] in Figure 2 for (a) $\eta=0$ and (b) $\eta=10^{-3}$ as function of electron-to-ion temperature ratio in the range $1 \leq T_{e} / T_{i} \leq 6$ and electron spectral index $1.6 \leq \kappa_{e} \leq 5$ at near-Maxwellian ions $\kappa_{i}=100$. Since the speed of the test charge is much lower than the dust thermal speed (viz., $v_{T} \ll v_{T d}$ ), the test charge is therefore screened by all the plasma species, viz., the electrons, ions, and dust grains, hence effectively modifying the shielding length. The effective shielding length is shown to be decreased in the dusty plasma in comparison with traditional electron-ion plasma and is strongly influenced by the suprathermal tails of electrons. The impact of the superthermal electrons at lower Kappa values is more effective in the case of electron-ion plasma than dusty plasma. In the plots, one can easily observe that light-colored regions correspond to higher values of the effective shielding length while dense-colored regions determine the lower values of the shielding length. However, in Figure 2(c) and (d), a reduction in the effective shielding length is revealed by the influence of suprathermal ions at near-Maxwellian electrons

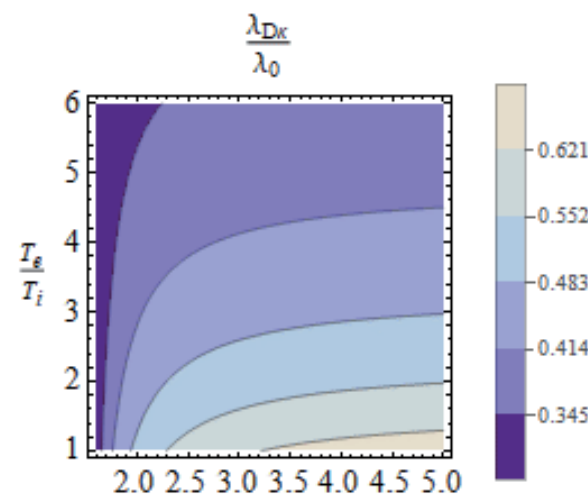

$K_{e}$

(a)
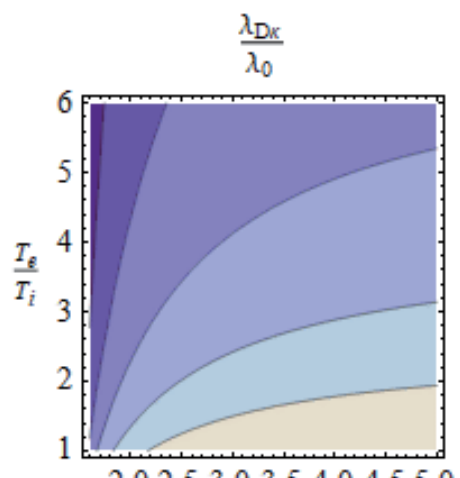

2.02 .53 .03 .54 .04 .55 .0

$K_{i}$

(c)

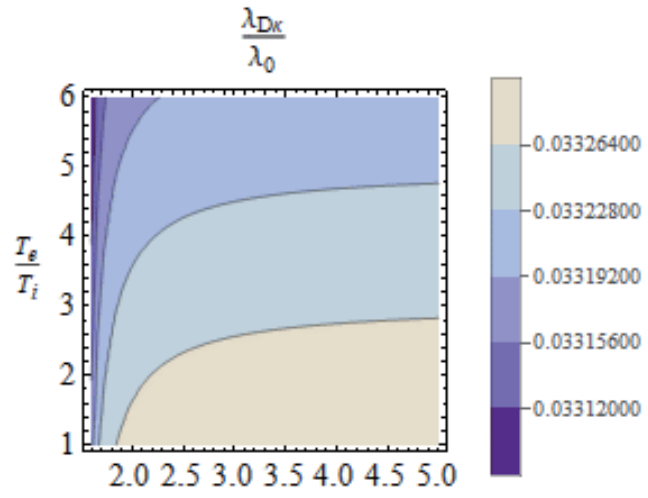

$K_{e}$

(b)

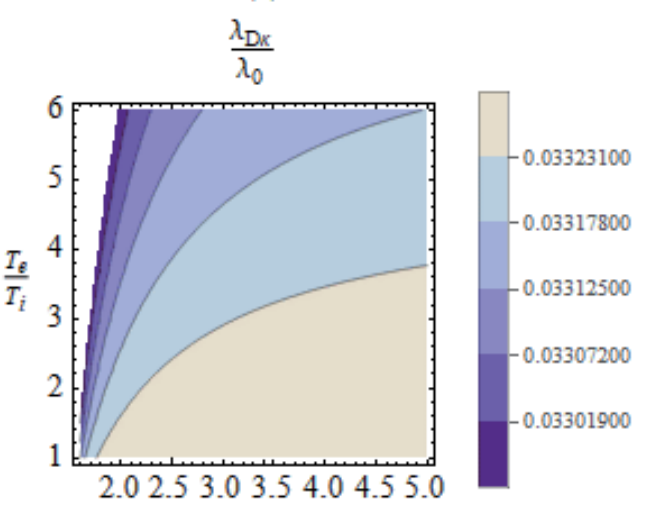

$K_{i}$

(d)

Figure 2.

Contours represent the effective shielding length $\left[\tilde{\lambda}_{D \kappa}=\lambda_{D \kappa} / \lambda_{\circ}\right]$ against the temperature ratio $\left(T_{e} / T_{i}\right)$ and electron spectral index $\left(\kappa_{e}\right)$ for $(a) \eta\left(=n_{d \circ} / n_{\circ}\right) \equiv 0$ (electron-ion plasma) and (b) $\eta=10^{-3}$ (dusty plasma) with $\kappa_{i}=100$ and $Z_{d o}=300$. contours in $(c)$ and $(d)$ vary for $\tilde{\lambda}_{D \kappa}$ against the temperature ratio $\left(T_{e} / T_{i}\right)$ and ion spectral index $\left(\kappa_{i}\right)$ for $(c) \eta=0$ (electron-ion plasma) and $(d) \eta=10^{-3}$ (dusty plasma) with $\kappa_{e}=100$ and $Z_{d o}=300$. Other parameters are mentioned in Section 1.4.1. 
$\kappa_{e}=100$. It may be noted that due to the variation of electron-to-ion temperature ratio against the ion spectral index, the magnitudes of effective shielding length become smaller at lower values of $\kappa_{i}$ than the case of $\kappa_{e}$. However, the effective shielding length approaches to the maximum value in the limit $T_{e} \simeq T_{i}$ and decreases by enhancing the electron-to-ion temperature ratios.

\subsection{Short-range DH and long-range FF potentials}

To study short and long-range shielded potentials of a slowly moving test charge along the $z$-axis in a Lorentzian dusty plasma, we use Eq. (9) into Eq. (7) and spherical polar coordinates, as $\mathbf{v}_{T}=\left(0,0, v_{T}\right), \mathbf{k}=\left(k \sin \theta_{k} \cos \varphi_{k}, \quad k \sin \theta_{k} \sin \varphi_{k}, \quad k \cos \theta_{k}\right)$, and $\mathbf{r}=\left(r \sin \theta_{r} \cos \varphi_{r}, r \sin \theta_{r} \sin \varphi_{r}, r \cos \theta_{r}\right)$ to finally obtain the total potential $\left[\phi_{1}(\mathbf{r}, t)=\phi_{D H}+\phi_{F F}\right]$ as

$$
\phi_{1}(\mathbf{r}, t)=\frac{q_{T}}{r} \exp \left(-\frac{r}{\lambda_{D \kappa}}\right)+\frac{2 \sqrt{2} q_{T}}{\sqrt{\pi} r} \frac{v_{T}}{v_{T d}} \frac{\xi \lambda_{D \kappa}}{\lambda_{D d}^{2}} \frac{\lambda_{D \kappa}^{3}}{r^{3}}
$$

The first part of Eq. (11) corresponds to the short-range Debye-Hückel (DH) potential, which accounts for the short distances between the test charge and observer, whereas the second part represents the long-range far-field potential in the limit $r \gg \lambda_{D \kappa}$ decaying as the inverse cube of the distance to the test charge. Here $r=\left(\rho^{2}+\xi^{2}\right)^{1 / 2}$ is the distance from the test charge to observer with radial and axial positions $\rho$ and $\xi\left(=z-v_{T} t\right)$, respectively. When $\kappa_{e, i} \rightarrow \infty$, the effective Debye length $\lambda_{D \kappa}$ approaches to $\lambda_{D}$ with $c_{\kappa e, i}=1$, and consequently Eq. (11) exactly coincides with the earlier result [36] in the limit $\cos (\gamma)=\xi / r$, having null dustcharge fluctuations. In Figure 3, the magnitude of the effective shielding length varies against the specific ranges of the electron concentration $\left(\mu_{e}\right)$ and dust concentration $(\eta)$ for changing the (a) electron-to-ion temperature ratios $\frac{T_{e}}{T_{i}}(=1,2,4,8)$ at near-Maxwellian electrons and ions $\kappa_{e, i}=100$ and (b) electron-to-dust temperature ratios $\frac{T_{e}}{T_{d}}(=10,15,20,25)$ with fixed $\frac{T_{e}}{T_{i}}=2$ for Kappa-distributed electrons and ions (i.e., $\kappa_{e, i}=1.6$ ), respectively. Note that for an electron-ion plasma, the effective shielding length is approached to unity [35] as the maximum value for isothermal case $\frac{T_{e}}{T_{i}}=1$ (black dotted curve) (see Figure 3(a)), which then decreases with respect to the electron concentration. For non-isothermal values, that is, $\frac{T_{e}}{T_{i}}=2$
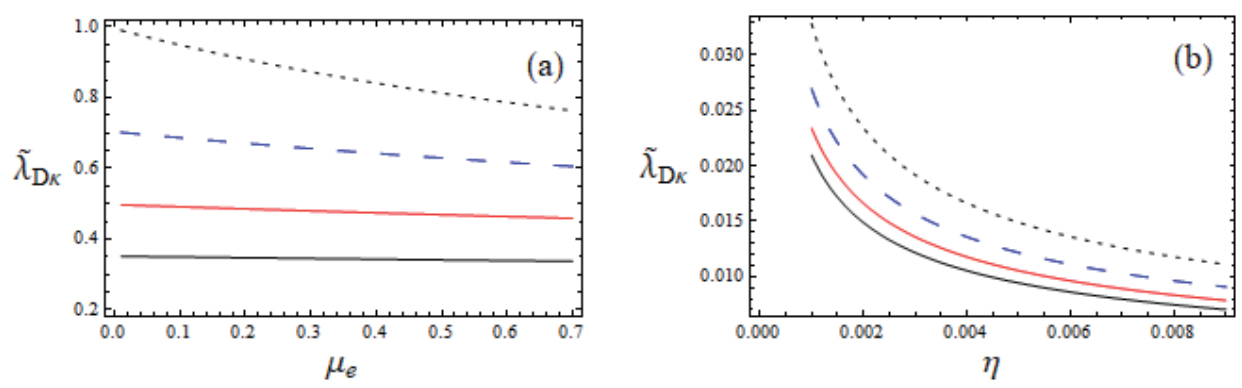

Figure 3.

The shielding length $\tilde{\lambda}_{D_{\kappa}}\left(=\lambda_{D \kappa} / \lambda_{\circ}\right)$ against the electron and dust concentrations $\left(\mu_{e}, \eta\right)$ for varying the temperature ratios $\left(\frac{T_{e}}{T_{i}}, \frac{T_{e}}{T_{d}}\right)$ in (a) an electron-ion Maxwellian plasma and $(b)$ superthermal dusty plasma, respectively. 
(blue dashed curve), 4 (red solid curve), and 8 (black solid curve), the strength of the effective shielding however reduces in terms of fraction showing no more dominant impact of the electron concentration. In the presence of dust component, the effective shielding length is reduced as compared to the electron-ion plasma and clearly depends on the electron-to-dust temperature ratios $\frac{T_{e}}{T_{d}}(=10,15,20,25)$ at $Z_{d 0}=300$, as can be seen in Figure 3(b).

The variation of the normalized DH potential $\tilde{\phi}_{D H}$ caused by a slowly moving test charge is displayed against the normalized axial distance $\tilde{\xi}$ for varying the dust concentration $\eta=10^{-3}, 1.2 \times 10^{-3}$, and $1.4 \times 10^{-3}$ in Figure 4 for (a) nonMaxwellian electrons and ions, as well as (b) near-Maxwellian electrons and ions. The DH potentials fastly reduce with a variation of dust concentration and attain large magnitudes in the near-Maxwellian case at $\kappa_{e, i}=100$ compared to nonMaxwellian case at $\kappa_{e, i}=1.6$.

Figure 5 exhibits how suprathermal electrons and ions modify the profiles of long-range FF potential $\left(\tilde{\phi}_{F F}\right)$ caused by a slow test charge moving with speed $v_{T}=$ $0.02 v_{T d}$. For small values of electron spectral index $\kappa_{e}(=1.6,1.8)$, the shielded FF potentials are more localized than the case at near-Maxwellian electrons for $\kappa_{e}=$ 100 (see the red solid curve in Figure 5(a)). However, the magnitudes of the FF potential are comparatively decreased in Figure 5(b) because of the strong contribution of suprathermal ions.
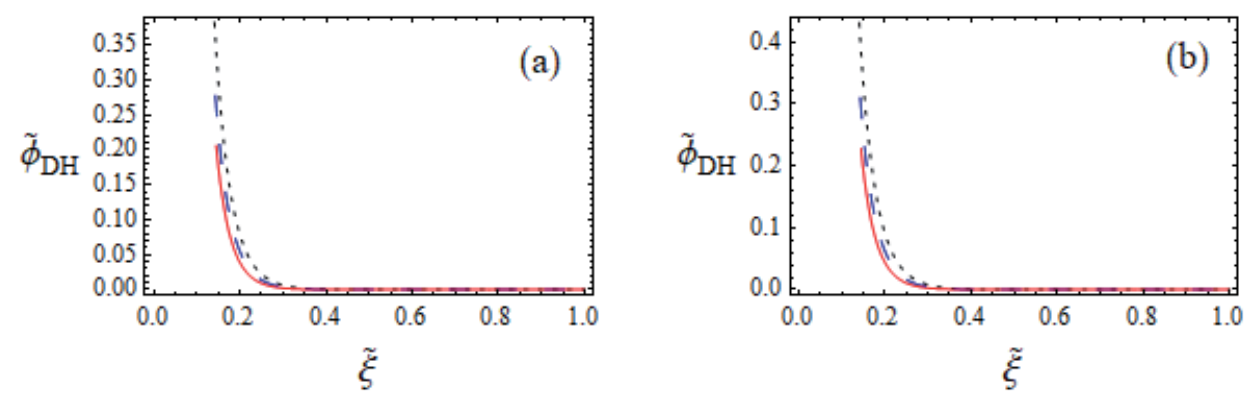

Figure 4.

The DH potential $\tilde{\phi}_{D H}\left[=\phi_{D H} /\left(q_{T} / \lambda_{0}\right)\right]$ vs. the axial distance $\tilde{\xi}\left(=\xi / \lambda_{0}\right)$ for different dust concentrations $\eta=$ $10^{-3}$ (black dotted curve), $1.2 \times 10^{-3}$ (blue dashed curve), and $1.4 \times 10^{-3}$ (red solid curve) at $Z_{d 0}=200$ and $\tilde{\rho}=0$ with $(a) \kappa_{e, i}=1.6$ and $(b) \kappa_{e, i}=100$.
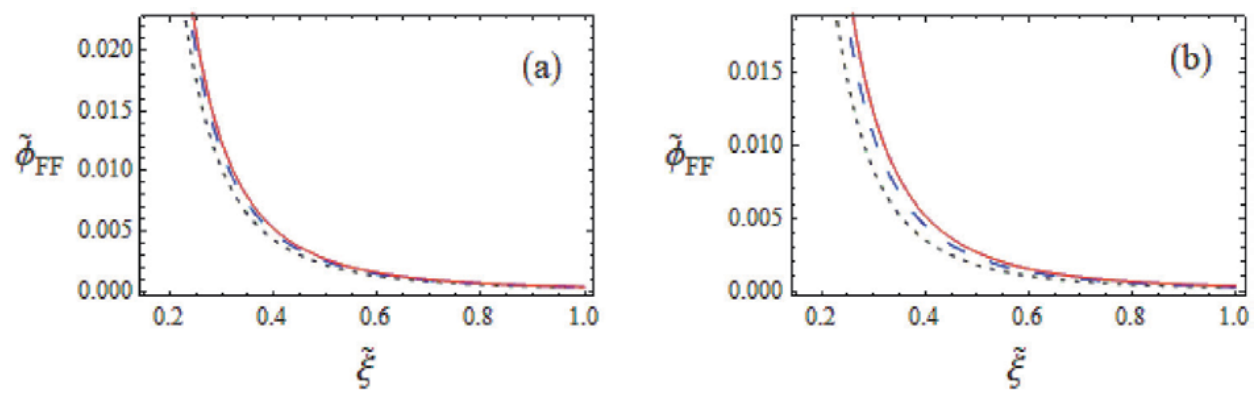

Figure 5.

The FF potential $\tilde{\phi}_{F F}\left[=\phi_{F F} /\left(q_{T} / \lambda_{0}\right)\right]$ vs. $\tilde{\xi}$ for varying (a) $\kappa_{e}=1.6$ (black dotted curve), 1.8 (blue dashed curve), and 100 (red solid curve) with $\kappa_{i}=100$, and $(b) \kappa_{i}=1.6$ (black dotted curve), 1.8 (blue dashed curve), and 100 (red solid curve) with $\kappa_{e}=100$. other values are $\eta=10^{-4}, \rho=0$, and $Z_{d 0}=300$. 


\subsection{Resonating test charge response}

To examine the resonant interaction of a test charge with DA waves, we first simply derive the dielectric constant of the DA waves using the limit $v_{T d} \ll \omega / k \ll \theta_{T e}, \theta_{T i}$ in Eq. (8). We therefore consider the small and large argument expansions $C_{s} \ll 1$ and $C_{d} \gg 1$ of the plasma dispersion functions. On DA scales, the inertia is mainly provided by the negatively charged dust grains and restoring force by the pressures of superthermal inertialess electrons and ions for maintaining the propagation of DA waves. In typical laboratory plasmas, the frequency of DA waves is often below the dust plasma oscillation frequency in the range of 1-100 Hz. Thus, the modified dielectric constant for a Lorentzian dusty plasma takes the following form

$$
D\left(k, \mathbf{k} \cdot \mathbf{v}_{T}\right)=1+\frac{1}{k^{2} \lambda_{D}^{\prime 2}}-\frac{\omega_{p d}^{2}}{\left(\mathbf{k} \cdot \mathbf{v}_{T}\right)^{2}}
$$

The effective Debye length now gets a new form $\lambda_{D}^{\prime}=\lambda_{0} / \sqrt{B_{\kappa}}$ with $B_{\kappa}=$ $c_{\kappa e} \frac{n_{e 0}}{n_{0}}+c_{\kappa i} \frac{T_{e}}{T_{i}}$. Note that dust-charge fluctuations are also ignored here because the characteristic damping rate attributed to dust charge fluctuations is much smaller than the collisional and Landau damping rates. The reciprocal of Eq. (12) can be simplified as

$$
D^{-1}=\frac{k^{2} \lambda_{0}^{2}}{B_{\kappa}+k^{2} \lambda_{0}^{2}}\left\{1+\frac{\omega_{D}^{2}}{\left(\mathbf{k} \cdot \mathbf{v}_{T}\right)^{2}-\omega_{D}^{2}}\right\},
$$

with

$$
\omega_{D}=\frac{k C_{D}}{\left(B_{\kappa}+k^{2} \lambda_{0}^{2}\right)^{1 / 2}} .
$$

This is the DA resonance frequency with DA speed $C_{D}\left(=\omega_{p d} \lambda_{0}\right)$. For long wavelength limit $k \lambda_{0} \ll B_{\kappa}$, the DA frequency reduces to $\omega_{D}=k C_{D} / \sqrt{B_{\kappa}}$ in the Lorentzian dusty plasma, while in the short wavelength limit $k \lambda_{0} \gg B_{\kappa}$, the frequency simply approaches to the dust plasma oscillation frequency $\omega_{D} \simeq \omega_{p d}$. The factor $\omega_{D}^{2} /\left\{\left(\mathbf{k} \cdot \mathbf{v}_{T}\right)^{2}-\omega_{D}^{2}\right\}$ in Eq. (13) identifies the dynamical effects of dust grains, which may lead to an oscillatory WF potential strongly depending upon whether the product $\mathbf{k} \cdot \mathbf{v}_{T}$ is smaller or larger than $\omega_{D}$.

\subsection{Short-range DH and long-range WF potentials}

For static or slowly moving test charge in a Lorentzian dusty plasma, its potential distributions are found spherically symmetric both in the axial and radial directions. Consequently, the DH and FF shielded potentials are appropriately solved with spherical polar coordinates. However, if the test charge moves with finite speed in a specific direction along the $\mathrm{z}$-axis, the resonant interaction of test charge with the DA wave leads to the asymmetric distribution of potential in the form of WF behind the test charge. The plasma model is then preferably solved in cylindrical coordinates. Thus, following the standard techniques [37, 38, 40] for $\mathrm{DH}$ and WF potentials, we make use of Eq. (13) into Eq. (7) to finally arrive at 


$$
\phi_{1}(\mathbf{r}, t)=\frac{q_{T}}{r} \exp \left(-\frac{r \sqrt{B_{\kappa}}}{\lambda_{0}}\right)+\frac{2 q_{T}}{\xi}\left(1+\frac{C_{D}^{2}}{B_{\kappa} v_{T}^{2}}\right)\left(B_{\kappa}-\frac{C_{D}^{2}}{v_{T}^{2}}\right)^{-1} \cos \left(\frac{\omega_{p d} \xi}{\sqrt{B_{\kappa}} v_{T}}\right),
$$

where $\xi\left(=r_{\|}-v_{T} t\right)$ is the axial distance between the test charge and observer. The first part on the right hand side of Eq. (15) shows the contribution of modified $\mathrm{DH}$ potential and the second part corresponds to oscillatory WF potential accounting for the suprathermal electrons and ions in a Lorentzian dusty plasma. For $v_{T}>C_{D}$ and $\cos \left(\omega_{p d} \xi / v_{T} \sqrt{B_{\kappa}}\right)<0$, the WF potential becomes attractive [40] and dominates over the repulsive $\mathrm{DH}$ potential because the latter decreases rapidly beyond the shielding cloud. An oscillatory WF potential $\tilde{\phi}_{W F}\left(=\phi_{W F} \lambda_{0} / q_{T}\right)$ of a test charge moving with speed $v_{T}=0.2 C_{D}$ is shown along the axial direction $\tilde{\xi}$ as a function of spectral indices $\kappa_{e}$ and $\kappa_{i}$ in Figure 6(a) and (b), respectively. Observe that the amplitude of the WF potential increases as the superthermal indices $\kappa_{e}$ and $\kappa_{i}$ increase at fixed $\eta=10^{-3}$ and $Z_{d 0}=300$. Moreover, the wakefield damps behind the test charge a bit earlier as shown in Figure 6(b) due to the strong dependence of suprathermal ions on the effective shielding length in comparison with the suprathermal electrons. The impact of electron-to-ion temperature ratio is also examined on the profiles of WF and DH potentials as a function of axial distance $\tilde{\xi}$

(a)

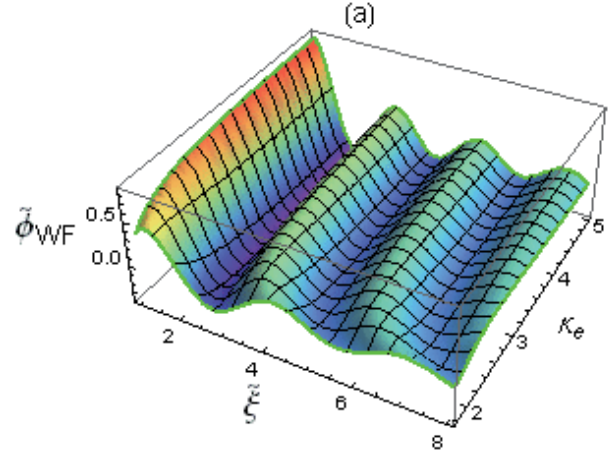

(b)

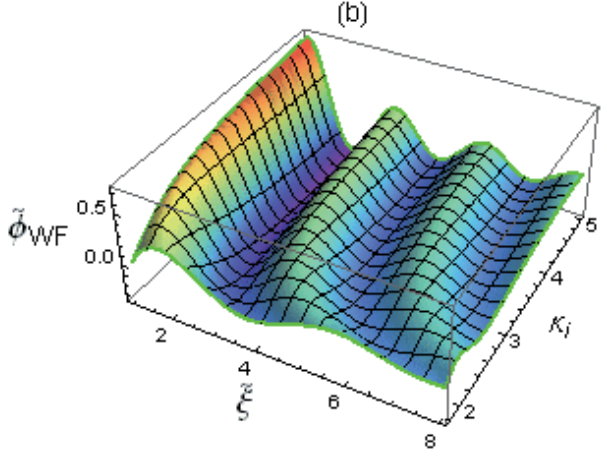

Figure 6.

The normalized WF potential $\tilde{\phi}_{W F}$ as a function of $\tilde{\xi}$ for varying the spectral indices as (a) $1.8 \leq \kappa_{e}<5$ with fixed $v_{T}=0.2 C_{D}$ and $\kappa_{i}=100$, and $(b) 1.8 \leq \kappa_{i}<5$ with $v_{T}=0.2 C_{D}$ and $\kappa_{e}=100$.
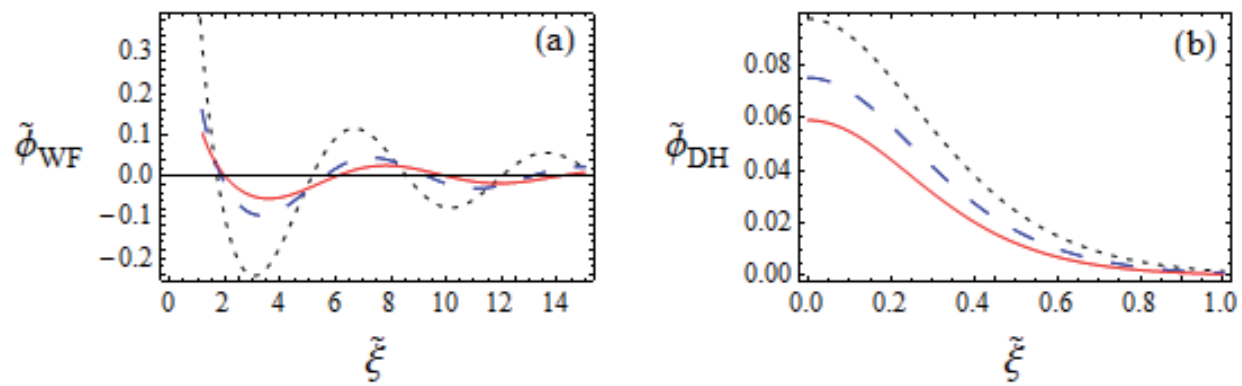

Figure 7.

The WF and DH potentials are shown against the axial distance $\tilde{\xi}$ for changing temperature ratios $(a) T_{e} / T_{i}=2$ (black dotted curve), 2.5 (blue dashed curve), and 3 (red solid curve), and (b) $T_{e} / T_{i}=2$ (black dotted curve), 2.5 (blue dashed curve), and 3 (red solid curve), respectively. Other common parameters are $\kappa_{e, i}=1.6$, $v_{T}=0.2 C_{D}, \eta=10^{-3}$, and $Z_{d o}=100$. 
with fixed radial distance $\rho=0.54 \lambda_{0}$ and $v_{T}=0.2 C_{D}$ as can be seen in Figure 7(a) and (b), respectively. It is important to examine that electron-to-ion temperature ratio suppresses the magnitudes of the WF and DH potentials at low values of $\kappa_{e, i}=1.6$ in the Lorentzian dusty plasma (Figures 6 and 7).

\subsection{Fast moving test charge response}

In this case, the test charge is assumed to be moving much faster than all the plasma species (viz., the electrons, ions and negatively charged dust grains). Consequently, Eq. (6) can be expressed in $1 \mathrm{D}$ form to finally arrive at $D\left(k, \mathbf{k} . \mathbf{v}_{T}\right) \simeq 1$ in the limits $v_{T} \mid \gg k u_{j}$ and $\omega=\mathbf{k} \cdot \mathbf{v}_{T}$. Thus, the test charge potential (7) simply leads to the Coulomb potential

$$
\phi_{1}(\mathbf{r}, t)=\phi_{C} \equiv \frac{q_{T}}{r} .
$$

It is now clear that if the test charge is moving very fast, then there is no shielding around it in the Lorentzian dusty plasma.

\section{Conclusion}

To conclude, we have briefly discussed different plasma diagnostic techniques and specifically investigated the novel features of interaction potentials caused by a test charge moving with constant velocity $\mathbf{v}_{T}$ along the $\mathrm{z}$-axis in a collisionless unmagnetized Lorentzian dusty plasma. For this purpose, the linearized coupled Vlasov-Poisson equations are employed to model suprathermal electrons and ions with Kappa-df, as well as negatively charged dust grains with Maxwell-df, respectively. After applying the space-time Fourier transformations, an electrostatic potential is obtained with a modified dielectric constant. For taking the test charge speed much smaller than the dust thermal speed in a Lorentzian dusty plasma, we then express the total potential distribution in terms of short-range Debye-Hückel (DH) and long-range far-field potentials. The DH potential exponentially decays with distance, whereas FF potential decreases as the inverse cube of the distance. Both the potentials are substantially influenced by the plasma and superthermality parameters. However, a resonating test charge with DA oscillations introduces the long-range WF potential excitations behind the test charge in Lorentzian dusty plasmas. A Coulomb potential is obtained when the test charge is moving very fast compared to plasma species, and there is no shielding around it in the Lorentzian dusty plasma.

Vladimirov and Nambu [40] have already utilized the idea of WF potential for making new materials by attracting the same polarity dust grains in dusty plasmas. The physics of attractive forces between the negatively charged dust grains is completely analogous to that of Cooper pairing of electrons in superconductors [59]. The dust particle physically polarizes the plasma medium and creates attractive potential regions, where positive ions from collective interaction of DA waves can be focused. This may in turn lead to the possibility for dust crystallization and dust coagulation in both laboratory and space dusty plasmas.

\section{Acknowledgements}

Dr. S. Ali dedicates this document to Late Prof. P.K. Shukla and Dr. B. Eliasson (University of Strathclyde, UK) who were very kind to him at many occasions 
during discussions on dusty plasmas, and acknowledges the partial financial assistance from USTC, Hefei, China, and ICTP, Trieste, Italy, for making his visits feasible in 2018 and 2019, respectively. Professor Y. Al-Hadeethi also acknowledges the technical support of the Deanship of Scientific Research (DSR), King Abdulaziz University, Saudi Arabia.

\section{Author details}

Shahid $\mathrm{Ali}^{1 *}$ and Yas Al-Hadeethi ${ }^{2}$

1 National Centre for Physics (NCP), Quaid-e-Azam University Campus, Islamabad, Pakistan

2 Faculty of Sciences, Department of Physics, King AbdulAziz University, Jeddah, Saudia Arabia

*Address all correspondence to: shahid_gc@yahoo.com

\section{IntechOpen}

(C) 2020 The Author(s). Licensee IntechOpen. This chapter is distributed under the terms of the Creative Commons Attribution License (http://creativecommons.org/licenses/ by/3.0), which permits unrestricted use, distribution, and reproduction in any medium, provided the original work is properly cited. (cc) BY 


\section{References}

[1] Al-Hadithi Y, Tallents GJ, Neely D. X-Ray Lasers 1990. Bristol: IOP Publishers; 1991

[2] Al-Hadithi Y, Tallents GJ, Zhang J, Key MH, Norreys PA, Kodama R. Energy transport in plasmas produced by a high brightness Krypton fluoride laser focused to a line. Physics of Plasmas. 1994;1:1279. DOI: 10.1063/ 1.870726

[3] Al-Hadeethi Y, Al-Mujtabi A, AlMarzouki FM. Laser produced plasma $\mathrm{x}$-ray sources for nanoscale resolution contact microscopy: A candidate in cancerous stem cells imaging. Advances in Molecular Imaging. 2017;7:67. DOI: 10.4236/ami.2017.74004

[4] Chen FF. Introduction to Plasma Physics and Controlled Fusion. New York: Plenum; 1984

[5] Fortov VE, Ivlev AV, Khrapak SA, Morfill GE. Complex (dusty) plasmas: Current status, open issues, perspectives. Physics Reports. 2005;421: 1. DOI: $10.1016 /$ j.physrep.2005.08.007

[6] Shukla PK, Silin VP. Dust ionacoustic wave. Physica Scripta. 1992;45: 508. DOI: 10.1088/0031-8949/45/5/015

[7] Rao NN, Shukla PK, Yu MY. Dustacoustic waves in dusty plasmas. Planetary Space Science. 1990;38:543. DOI: 10.1016/0032-0633(90)90147-I

[8] Barkan A, D’Angelo N, Merlino RL. Experiments on ion-acoustic waves in dusty plasmas. Planetary Space Science. 1996;44:239. DOI: 10.1016/0032-0633 (95) 00109-3

[9] Merlino RL, Barkan A, Thompson C, D'Angelo N. Laboratory studies of waves and instabilities in dusty plasmas. Physics of Plasmas. 1998;5:1607. DOI: 10.1063/1.872828
[10] Barkan A, Merlino RL, D’Angelo N. Laboratory observation of the dustacoustic wave mode. Physics of Plasmas. 1995;2:3563. DOI: 10.1063/1.871121

[11] Krall NA, Trivelpiece AW.

Principles of Plasma Physics. New York: McGraw-Hill; 1973

[12] Neufeld J, Ritchie RH. Passage of charged particles through plasma. Physical Review. 1955;98:1632. DOI: 10.1103/PhysRev.98.1632

[13] Montgomery D, Joyce G, Sugihara R. Inverse third power law for the shielding of test particles. Plasma Physics. 1968;10:681. DOI: 10.1088/ 0032-1028/10/7/304

[14] Stenflo L, Yu MY, Shukla PK. Shielding of a slow test charge in a collisional plasma. Physics of Fluids. 1973;16:450. DOI: 10.1063/1.1694361

[15] Shukla PK, Spatschek K-H. Shielding of a moving test charge in a turbulent plasma. Physics Letters A. 1973;44:398. DOI: 10.1016/0375-9601 (73) $90840-2$

[16] Yu MY, Stenflo L, Shukla PK. On the electric field of a moving test charge. Radio Science. 1972;7:1151. DOI: 10.1029/RS007i012p01151

[17] Shukla PK, Spatschek K-H, Yu MY. Far-field potential of a test charge in an inhomogeneous and magnetized plasma. Canadian Journal of Physics. 1974;52: 281. DOI: $10.1139 / \mathrm{p} 74-040$

[18] Ali S, Nasim MH, Murtaza G. Effects of dust-charge fluctuations on the potential of an array of projectiles in a partially ionized dusty plasma. Physics of Plasmas. 2003;10:4207. DOI: 10.1063/ 1.1619976

[19] Ali S, Nasim MH, Murtaza G. Correlation effects due to an axial 
propagation of projectiles in a dusty plasma. Physics of Plasmas. 2005;12: 033502. DOI: $10.1063 / 1.1844496$

[20] Ali S, Murtaza G, Nasim MH. Energy loss for the assemblies of charged projectiles in a dusty plasma. Physics of Plasmas. 2005;12:072104.

DOI: 10.1063/1.1933739

[21] Brandt W, Ratkowski A, Ritchie RH. Energy loss of swift proton clusters in solids. Physical Review Letters. 1974;33: 1325. DOI: 10.1103/PhysRevLett.33.1325

[22] Deutsch C, Tahir NA.

Fragmentation and stopping of heavy cluster ions in a lithium targetApplication to target implosion. Physics of Fluids B. 1992;4:3735. DOI: 10.1063/ 1.860329

[23] Skupsky S. Energy loss of ions moving through high-density matter. Physical Review A. 1977;16:727. DOI: 10.1103/PhysRevA.16.727

[24] Li CK, Petrasso RD. Chargedparticle stopping powers in inertial confinement fusion plasmas. Physical Review Letters. 1993;70:3059

[25] Lindl JD. Inertial Confinement Fusion. New York: Springer Verlag; 1998

[26] Jones ME, Keinigs R. Ion plasma wave wakefield accelerators. IEEE Transactions on Plasma Science. 1987; 15:203. DOI: 10.1109/TPS.1987.4316686

[27] Mohideen U, Rahman HU, Smith MA, Rosenberg M, Memdis DA. Intergrain coupling in dusty-plasma Coulomb crystals. Physical Review Letters. 1998;81:349. DOI: 10.1103/ PhysRevLett.81.349

[28] Takahashi K, Oishi T, Shimomai K-I, Hayashi Y, Nishino S. Analyses of attractive forces between particles in Coulomb crystal of dusty plasmas by optical manipulations. Physical
Review E. 1998;58:7805. DOI: 10.1103/ PhysRevE.58.7805

[29] Zwicknagel G, Deutsch C. Correlated ion stopping in plasmas. Physical Review E. 1997;56:970. DOI: 10.1103/PhysRevE.56.970

[30] Sanmartin JR, Lam SH. Far-wake structure in rarefield plasma flows past charged bodies. Physics of Fluids. 1971; 14:62. DOI: $10.1063 / 1.1693289$

[31] Yu MY, Tegeback R, Stenflo L. On test charge potentials in collisional plasmas. Zeitschrift für Physik. 1973;

264:341. DOI: 10.1007/BF01398860

[32] Peter T. Linearized potential of an ion moving through plasma. Journal of Plasma Physics. 1990;44:269. DOI: $10.1017 /$ S0022377800015178

[33] Shivamoggi BK, Mulser P. Dielectric screening and stopping power of a test charge moving in a plasma. Journal of Plasma Physics. 1998;60:819

[34] Shukla PK, Singh RN. Total energy loss of a test charge in a collisional magnetoplasma. Physica Scripta. 1971;4: 282. DOI: $10.1088 / 0031-8949 / 4 / 6 / 008$

[35] Lakshmi VS, Bharuthram R, Shukla PK. Debye shielding in a dusty plasma. Astrophysics and Space Science. 1993;209:213. DOI: 10.1007/ BF00627441

[36] Shukla PK. Shielding of a slowly moving test charge in dusty plasmas. Physics of Plasmas. 1994;1:1362. DOI: 10.1063/1.870736

[37] Nambu M, Akama H. Attractive potential between resonant electrons. Physics of Fluids. 2300;1985:28. DOI: 10.1063/1.865284

[38] Nambu M, Vladimirov SV, Shukla PK. Attractive forces between charged particulates in plasmas. Physics 
Letters A. 1995;203:40. DOI: 10.1016/ 0375-9601(95)00380-L

[39] Shukla PK, Rao NN. Coulomb crystallization in colloidal plasmas with streaming ions and dust grains. Physics of Plasmas. 1996;3:1770. DOI: 10.1063/ 1.871695

[40] Vladimirov SV, Nambu M. Attraction of charged particles in plasmas with finite flows. Physical Review E. 1995;52:R2172. DOI: 10.1103/ PhysRevE.52.R2172

[41] Nambu M, Saikia BJ, Hada T. Wake potential around a test dust particulate in a magnetized plasma with streaming ions. Journal of the Physical Society of Japan. 2001;70:1175. DOI: 10.1143/ jpsj.70.1175

[42] Freund HP, Smith RA, Papadopoulos K, Palmadesso P. Modulational instability in a plasma with supra-thermal electrons. Physics of Fluids. 1981;24:442. DOI: 10.1063/ 1.863390

[43] Freese KB, Walsh JE, Lohr J. Waveparticle interaction in the late beamplasma instability. Physics of Fluids. 1979;22:2367. DOI: 10.1063/1.862549

[44] Smith RA, Goldstein ML, Papadopoulos K. Nonlinear stability of solar type III radio bursts. I-Theory. The Astrophysical Journal. 1979;234:348

[45] Van Compernolle B, Gekelman W, Pribyl P. Generation of supra-thermal electrons and Alfvén waves by a high power pulse at the electron plasma frequency. Physics of Plasmas. 2006;13: 092112. DOI: $10.1063 / 1.2261850$

[46] Eliasson B, Shao X, Milikh G, Mishin EV, Papadopoulos K. Numerical modeling of artificial ionospheric layers driven by high-power HF heating. Journal of Geophysical Research. 2012; 117:A10321. DOI: 10.1029/ 2012JA018105
[47] Vasyliunas VM. A survey of lowenergy electrons in the evening sector of the magnetosphere with OGO 1 and OGO 3. Journal of Geophysical Research. 1968;73:2839. DOI: 10.1029/ JA073i009p02839

[48] Summers D, Thorne RM. The modified plasma dispersion function. Physics of Fluids B. 1991;3:1835. DOI: 10.1063/1.859653

[49] Baluku TK, Hellberg MA. Dust acoustic solitons in plasmas with kappadistributed electrons and/or ions.

Physics of Plasmas. 2008;15:123705.

DOI: $10.1063 / 1.3042215$

[50] Ali S, Eliasson B. Slowly moving test charge in two-electron component nonMaxwellian plasma. Physics of Plasmas. 2015;22:084508. DOI: 10.1063/ 1.4928901

[51] Ali S, Eliasson B. Slow test charge response in a dusty plasma with kappa distributed electrons and ions. Physica Scripta. 2017;92:084003. DOI: 10.1088/ 1402-4896/aa7c09

[52] Burton FD, Conte SD. The Plasma Dispersion Function. New York:

Academic Press; 2015

[53] Debye P, Hückel E. On the Debye length in strong electrolytes.

Physikalische Zeitschrift. 1923;24:185

[54] Rubab N, Murtaza G. Debye length in non-Maxwellian plasmas. Physica Scripta. 2006;74:145. DOI: 10.1088/ 0031-8949/74/2/001

[55] Du J, Guo R, Liu Z, Du S. Slowing down of charged particles in dusty plasmas with power-law kappadistributions. Contributions to Plasma Physics. 2019;59:144. DOI: 10.1002/ ctpp.201800046

[56] Wahlund J-E, Andre M, Eriksson AIE, Lundberg M, Morooka MW, Shafiq M, et al. Detection of dusty plasma near the 
E-ring of Saturn. Planetary and Space Science. 2009;57:1795. DOI: 10.1016/j. pss.2009.03.011

[57] Leisner JS, Russell CT, Dougherty MK, Blanco-Cano X, Strangeway RJ, Bertucci C. Ion cyclotron waves in Saturn's E ring: Initial Cassini observations. Geophysical Research Letter. 2006;33:L11101. DOI: 10.1029/2005GL024875

[58] Kurth WS, Averkamp TF, Gurnett DA, Wang Z. Cassini RPWS observations of dust in Saturn's E Ring. Planetary and Space Science. 2006;54: 988. DOI: 10.1016/j.pss.2006.05.011

[59] De Gennes PG. Superconductivity of Metals and Alloys. New York: Benjamin; 1966 

Section 6

\section{Nonlinear Phenomena in Plasma}





\title{
Approximate Analytical Solution of Nonlinear Evolution Equations
}

\author{
Laxmikanta Mandi, Kaushik Roy and Prasanta Chatterjee
}

\begin{abstract}
Analytical solitary wave solution of the dust ion acoustic waves (DIAWs) is studied in the frame-work of Korteweg-de Vries (KdV), damped force Korteweg-de Vries (DFKdV), damped force modified Korteweg-de Vries (DFMKdV) and damped forced Zakharov-Kuznetsov (DFZK) equations in an unmagnetized collisional dusty plasma consisting of negatively charged dust grain, positively charged ions, Maxwellian distributed electrons and neutral particles. Using reductive perturbation technique (RPT), the evolution equations are obtained for DIAWs.
\end{abstract}

Keywords: solitary wave, soliton, KdV, DKdV, DFZK

\section{Introduction}

In the field of physics and applied mathematics research getting an exact solution of a nonlinear partial differential equation is very important. The elaboration of many complex phenomena in fluid mechanics, plasma physics, optical fibers, biology, solid-state physics, etc. is possible if analytical solutions can be obtained. Most of the differential equation arises in these field has no explicit solution as popularly known. This problem creates hindrances in the study of nonlinear phenomena and makes it time-consuming in the research of nonlinear models in the plasma and other science. However recent researches in nonlinear differential equations have seen the development of many approximate analytical solutions of partial and ordinary differential equations.

The history behind the discovery of soliton is not only interesting but also significant. In 1834 a Scottish scientist and engineer-John Scott-Russell first noticed the solitary water wave on the Edinburgh Glasgow Canal. In 1844 [1] in "Report on Waves" he accounted his examinations to the British Association. He wrote "I was observing the motion of a boat which was rapidly drawn along a narrow channel by a pair of horses, when the boat suddenly stopped not so the mass of water in the channel which it had put in motion; it accumulated round the prow of the vessel in a state of violent agitation, then suddenly leaving it behind, rolled forward with great velocity, assuming the form of a large solitary elevation, a rounded, smooth and well defined heap of water, which continued its course along the channel apparently without change of form or diminution of speed. I followed it on horseback and overtook it still rolling on at a rate of some eight or nine miles an hour, preserving its original figure some thirty feet long and a foot to a foot and a half in height. Its height gradually diminished and after a chase of one or two miles I lost it in the windings of the channel. Such in the month of August 1834 was my first 
chance interview with that singular and beautiful phenomenon which I have called the Wave of Translation." He coined the word "solitary wave." The solitary wave is called so because it often occurs as a single entity and is localized. The most important characteristics of solitary waves were unearthed after thorough study along with extensive wave-tank experiments. The following are the properties of solitary waves:

(a) These localized bell-shaped waves travel with enduring form and velocity. The speed of these waves are given by $c^{2}=g(h+a)$, where $g, a, h$ are respectively represent the acceleration of the gravity, amplitude of the wave and the undisturbed depth of the water. (b) Solitary waves can cross each other without any alteration.

John Scott-Russell's study created a stir in the scientific community. His study not only initiated a debate with the prevailing knowledge of the theories of waves but also challenged the antecedent knowledge of waves. The previous study claimed that a periodic wave of finite amplitude and permanent shape are feasible only in deep water unlike Russell's observation that the permanent profile is also possible in shallow water. Finally the stable form of solitary waves was received in scientific community with the aid of nonlinearity and dispersion. An ideal equilibrium between nonlinearity and dispersion can generate such waves.

Diederik Johannes Korteweg in 1895 [2] along with his PhD student Gustav De Vries obtained an equation from the primary equation of hydrodynamics. This equation explains shallow water waves where the existence of solitary waves was mathematically recognized. This equation is called $\mathrm{KdV}$ equation which is of the form $\frac{\partial u}{\partial t}+A u \frac{\partial u}{\partial x}+B \frac{\partial^{3} u}{\partial x^{3}}=0$. One of the most popular equations of soliton theory, this equation helps in explaining primary ideas that lie behind the soliton concept.

Martin Zabusky and Norman Kruskal [3] in 1965 solved KdV equation numerically and noticed that the localized waves retain their shape and momentum in collisions. These waves were known as "solitons." Soliton are solitary waves with the significant property that the solitons maintain the form asymptotically even when it experiences a collision. The fundamental "microscopic" properties of the soliton interaction; (i) the interaction does not change the soliton amplitudes; (ii) after the interaction, each soliton gets an additional phase shift; (iii) the total phase shift of a soliton acquired during a certain time interval can be calculated as a sum of the elementary phase shifts in pair wise collisions of this soliton with other solitons during this time interval is of importance. Solitons are mainly used in fiber optics, optical computer etc. which has really generated a stir in today's scientific community. The conventional signal dispensation depends on linear system and linear systems. After all in this case nonlinear systems create more well-organized algorithms. The optical soliton is comparatively different from KdV solitons. Unlike the $\mathrm{KdV}$ soliton that illustrates the wave in a solitary wave, the optical soliton in fibers is the solitary wave of an envelope of a light wave. In this regard, the optical soliton in a fiber is treated as an envelope soliton.

This chapter will discuss the analytical solitary wave solution of the KdV and KdV-like equations. In the study of nonlinear dispersive waves, these equations are generally seen. The KdV equation, a generic equation, is important in the study of weakly nonlinear long waves. This equation consists of a single humped wave characterized by several unique properties. The Soliton solutions of the KdV equation have been quite popular but it also not devoid of problems. The problems not only restrict to dispersion but also dissipation and interestingly these are not dominated by the KdV equation. The standard KdV equation fails to explain the development of small-amplitude solitary waves in case the particles collide in a plasma system. KdV equation with an additional damping term or the damped 
Korteweg-de Vries (DKdV) equation becomes handy in explaining this issue of elaborating the character of the wave. But in the presence of any critical physical situation (critical point) nonlinearity of the KdV equation disappears and the amplitude of the waves reaches infinity. To control this situation, a new nonlinear partial differential equation has to be derived that can explain the system at that critical point. This is known as the modified Korteweg-de Vries (MKdV) equation. In the presence of collisions, this equation is not also adequate and a damped MKdV equation is necessary. Also in the presence of force source term then the equation will be further modified and become DFKdV/DFMKdV.

\section{The Korteweg-de Vries equation}

Now we will derive the KdV equation from a classic plasma model, in which we consider a collision-free unmagnetized plasma consists of electrons and ions, in which ions are mobile and electrons obey the Maxwell distribution. The basic equation will be given as:

$$
\begin{gathered}
\frac{\partial N_{i}}{\partial T}+\frac{\partial N_{i} U_{i}}{\partial X}=0 \\
\frac{\partial U_{i}}{\partial T}+U_{i} \frac{\partial U_{i}}{\partial X}=-\frac{e}{m_{i}} \frac{\partial \psi}{\partial X} \\
\varepsilon_{0} \frac{\partial^{2} \psi}{\partial X^{2}}=e\left(N_{e}-N_{i}\right)
\end{gathered}
$$

where the electrons obey Maxwell distribution, i.e., $N_{e}=e n_{0} e^{\frac{e \phi}{K_{B} T_{e}}} \cdot N_{i}, N_{e}, U_{i}, m_{i}$ are the ion density, electron density, ion velocity and ion mass, respectively. $\psi$ is the electrostatic potential, $K_{B}$ is the Boltzmann constant, $T_{e}$ is the electron temperature and $e$ is the charge of the electrons.

To write Eqs. (1)-(3) in dimensionless from we introduce the following dimensionless variables

$$
x=\frac{X}{\lambda_{D}}, t=\omega_{p} T, \phi=\frac{e \psi}{K T_{e}}, n_{i}=\frac{N_{i}}{n_{0}}, u_{i}=\frac{U_{i}}{c_{s}},
$$

where $\lambda_{D}=\sqrt{\varepsilon_{0} K_{B} T_{e} / n_{0} e^{2}}$ is the Debye length, $c_{s}=\sqrt{K_{B} T_{e} / m_{i}}$ is the ion acoustic speed, $\omega_{p i}=\sqrt{n_{0} e^{2} / \varepsilon_{0} m_{i}}$ is the ion plasma frequency and $n_{0}$ is the unperturbed density of ions and electrons. Hence using (4) in (1)-(3) we obtain the normalized set of equations as

$$
\begin{gathered}
\frac{\partial n_{i}}{\partial t}+\frac{\partial\left(n_{i} u_{i}\right)}{\partial x}=0 \\
\frac{\partial u_{i}}{\partial t}+u_{i} \frac{\partial u_{i}}{\partial x}=-\frac{\partial \phi}{\partial x} \\
\frac{\partial^{2} \phi}{\partial x^{2}}=e^{\phi}-n_{i}
\end{gathered}
$$

To linearized (5)-(7), let us write the dependent variable as sum of equilibrium and perturbed parts, so that we write $n_{i}=1+\bar{n}_{i}, u_{i}=\bar{u}_{i}, \phi=\bar{\phi}$. Putting $n_{i}=1+\bar{n}_{i}$ where the values of parameters at equilibrium position is given by $n_{1}=1, u_{1}=0$ and $\phi_{i}=0$ in Eq. (5), we get 


$$
\frac{\partial}{\partial t}\left(1+\bar{n}_{i}\right)+\frac{\partial}{\partial x}\left(\bar{u}_{i}+\bar{n}_{i} \bar{u}_{i}\right)=0
$$

neglecting the nonlinear term $\frac{\partial\left(\bar{n}_{i} \bar{u}_{i}\right)}{\partial x}$ from (8), we get

$$
\frac{\partial \bar{n}_{i}}{\partial \bar{t}}+\frac{\partial \bar{u}_{i}}{\partial \bar{x}}=0
$$

which is the linearized form of Eq. (5).

Putting $u_{i}=\bar{u}_{i}, \phi=\bar{\phi}$ in Eq. (6), we get

$$
\frac{\partial \bar{u}_{i}}{\partial t}+\bar{u}_{i} \frac{\partial \bar{u}_{i}}{\partial x}=-\frac{\partial \bar{\phi}}{\partial x}
$$

Neglecting the nonlinear term from (10), we get

$$
\frac{\partial \bar{u}_{i}}{\partial t}+\frac{\partial \bar{\phi}}{\partial \bar{x}}=0
$$

This is the linearized form of Eq. (6).

Putting $n_{i}=1+\bar{n}_{i}, \phi=\bar{\phi}$ in Eq. (7), we get

$$
\begin{aligned}
& \frac{\partial^{2} \bar{\phi}}{\partial x}=1+\bar{\phi}-1-\bar{n}_{i} \\
& \Rightarrow \frac{\partial^{2} \bar{\phi}}{\partial x}=\bar{\phi}-\bar{n}_{i}
\end{aligned}
$$

Hence Eqs. (9), (11), (12) are the linearized form of Eq. (5)-(7) respectively.

To get dispersion relation for low frequency wave let us assume that the perturbation is proportional to $e^{i(k x-\omega t)}$ and of the form

$$
\begin{aligned}
& \bar{n}=n_{0} e^{i(k x-\omega t)} \\
& \bar{u}=u_{0} e^{i(k x-\omega t)} \\
& \bar{\phi}=\phi_{0} e^{i(k x-\omega t)}
\end{aligned}
$$

So,

$$
\begin{gathered}
\frac{\partial \bar{n}}{\partial t}=-i n_{0} \omega e^{i(k x-\omega t)} \\
\frac{\partial \bar{n}}{\partial x}=i k n_{0} e^{i(k x-\omega t)} \\
\frac{\partial \bar{u}}{\partial t}=-i u_{0} \omega e^{i(k x-\omega t)} \\
\frac{\partial \bar{u}}{\partial x}=i k u_{0} e^{i(k x-\omega t)} \\
\frac{\partial \bar{\phi}}{\partial x}=i k \phi_{0} e^{i(k x-\omega t)} \\
\frac{\partial^{2} \bar{\phi}}{\partial x^{2}}=(i k)^{2} \phi_{0} e^{i(k x-\omega t)}
\end{gathered}
$$


Putting these value in Eqs. (9), (11) and (12), we get,

$$
\begin{gathered}
-i \omega n_{0}+i k u_{0}=0 \\
-i \omega u_{0}+i k \phi_{0}=0 \\
n_{0}-\left(k^{2}+1\right) \phi_{0}=0
\end{gathered}
$$

Since the system (22)-(24) is a system of linear homogeneous equation so for nontrivial solutions we have

$$
\begin{gathered}
\left|\begin{array}{ccc}
-i \omega & i k & 0 \\
0 & -i \omega & i k \\
1 & 0 & -\left(k^{2}+1\right)
\end{array}\right|=0 \\
\Rightarrow-i^{2} \omega^{2}\left(k^{2}+1\right)+i^{2} k^{2}=0 \\
\Rightarrow \omega^{2}\left(k^{2}+1\right)=-i^{2} k^{2} \\
\Rightarrow \omega^{2}=\frac{k^{2}}{\left(k^{2}+1\right)}
\end{gathered}
$$

This is the dispersion relation.

For small $k$, i.e., for weak dispersion we can expand as

$$
\begin{aligned}
\omega & =k\left(1+k^{2}\right)^{-\frac{1}{2}} \\
& =k-\frac{1}{2} K^{3}+\cdots
\end{aligned}
$$

The phase velocity as

$$
V_{p}=\frac{\omega}{k}=\frac{1}{\sqrt{\left(1+k^{2}\right)}}
$$

so that $V_{p} \rightarrow 1$ as $k \rightarrow 0$ and $V_{p} \rightarrow 0$ as $k \rightarrow \infty$. The group velocity $V_{g}=\frac{d w}{d k}$ is given by

$$
V_{g}=\frac{1}{\left(1+k^{2}\right)^{3 / 2}}
$$

In this case, we have $V_{g}<V_{p}$ for all $k>0$. The group velocity is more important as energy of a medium transfer with this velocity.

For long-wave as $k \rightarrow 0$, the leading order approximation is $\omega=k$, corresponding to non-dispersive acoustic waves with phase speed $\omega / k=1$. Hence this speed is the same as the speed of the ion-acoustic waves $c_{s}$. The long wave dispersion is weak, i.e., $k \lambda_{D}<<1$. This means that the wavelength is much larger than the Debye length. In these long waves, the electrons oscillate with the ions. The inertia of the wave is provided by the ions and the restoring pressure force by the electrons. At the next order in $k$, we find that

$$
\omega=k-\frac{1}{2} k^{3}+O\left(k^{5}\right) \quad \text { as } k \rightarrow 0
$$


The $O\left(k^{5}\right)$ correction corresponds to weak KdV type long wave dispersion. For short wave $(k \rightarrow \infty)$, the frequency $\omega=1$, corresponding to the ion plasma frequency $\omega_{p i}=\frac{c_{s}}{\lambda_{D}}$. Hence the ions oscillate in the fixed background of electrons.

Now the phase of the waves can be written as

$$
k x-\omega t=k(x-t)+\frac{1}{2} k^{3} t
$$

Here $k(x-t)$ and $k^{3} t$ have same dynamic status (dimension) in the phase. Assuming $k$ to be small order of $\varepsilon^{1 / 2}, \varepsilon$ being a small parameter measuring the weakness of the dispersion, Here $(x-t)$ is the traveling wave form and time $t$ is the linear form.

Let us consider a new stretched coordinates $\xi, \tau$ such that

$$
\xi=\varepsilon^{1 / 2}(x-\lambda t), \tau=\varepsilon^{3 / 2} t
$$

where $\varepsilon$ is the strength of nonlinearity and $\lambda$ is the Mach number (phase velocity of the wave). $\varepsilon$ may be termed as the size of the perturbation. Let the variables be perturbed from the stable state in the following way (considering $n_{i}=1, u_{i}=0$, $\phi=0$ and $n_{e}=e^{\phi}=e^{0}=1$ at equilibrium)

$$
\begin{aligned}
& n_{i}=1+\varepsilon n_{i}^{(1)}+\varepsilon^{2} n_{i}^{(2)}+\varepsilon^{3} n_{i}^{(3)}+\cdots, \\
& u_{i}=0+\varepsilon u_{i}^{(1)}+\varepsilon^{2} u_{i}^{(2)}+\varepsilon^{3} u_{i}^{(3)}+\cdots, \\
& \phi=0+\varepsilon \phi^{(1)}+\varepsilon^{2} \phi^{(2)}+\varepsilon^{3} \phi^{(3)}+\cdots
\end{aligned}
$$

where $x$ and $t$ are function of $\xi$ and $\tau$ so partial derivatives with respect to $x$ and $t$ can be transform into partial derivative in terms of $\xi$ and $\tau$ so

$$
\begin{gathered}
\frac{\partial}{\partial x}=\frac{\partial}{\partial \xi} \frac{\partial \xi}{\partial x}+\frac{\partial}{\partial \tau} \frac{\partial \tau}{\partial x}, \Rightarrow \frac{\partial}{\partial x}=\varepsilon^{\frac{1}{2}} \frac{\partial}{\partial \xi} \\
\frac{\partial}{\partial t}=\frac{\partial}{\partial \xi} \frac{\partial \xi}{\partial t}+\frac{\partial}{\partial \tau} \frac{\partial \tau}{\partial t}, \Rightarrow \frac{\partial}{\partial t}=-\varepsilon^{\frac{1}{2}} \frac{\partial}{\partial \xi}+\varepsilon^{\frac{3}{2}} \frac{\partial}{\partial \tau} \\
\frac{\partial^{2}}{\partial x^{2}}=\frac{\partial}{\partial x}\left(\varepsilon^{\frac{1}{2}} \frac{\partial}{\partial \xi}\right), \Rightarrow \frac{\partial^{2}}{\partial x^{2}}=\varepsilon \frac{\partial^{2}}{\partial \xi^{2}}
\end{gathered}
$$

We can express (5)-(7) in terms of $\xi$ and $\tau$ as

$$
\begin{gathered}
\varepsilon^{3 / 2} \frac{\partial n_{i}}{\partial \tau}-\varepsilon^{1 / 2} \lambda \frac{\partial n_{i}}{\partial \xi}+\varepsilon^{1 / 2} \frac{\partial\left(n_{i} u_{i}\right)}{\partial \xi}=0 \\
\varepsilon^{3 / 2} \frac{\partial u_{i}}{\partial \tau}-\varepsilon^{1 / 2} \lambda \frac{\partial u_{i}}{\partial \xi}+\varepsilon^{1 / 2} u_{i} \frac{\partial u_{i}}{\partial x}=-\varepsilon^{1 / 2} \frac{\partial \phi}{\partial x} \\
\varepsilon \frac{\partial^{2} \phi}{\partial \xi^{2}}=e^{\phi}-n_{i}
\end{gathered}
$$

Substituting the Eqs. (31)-(34) in Eqs. (38)-(40) and collecting the lowest order $O\left(\varepsilon^{3 / 2}\right)$ terms we get

$$
-\lambda \frac{\partial n_{i}^{(1)}}{\partial \xi}+\frac{\partial u_{i}^{(1)}}{\partial \xi}=0
$$




$$
\begin{gathered}
-\lambda \frac{\partial u_{i}^{(1)}}{\partial \xi}=\frac{\partial \phi^{(1)}}{\partial \xi}, \\
\phi^{(1)}-n_{i}^{(1)}=0 .
\end{gathered}
$$

Integrating Eqs. (41)-(43) and all the variables tend to zero as $\xi \rightarrow \infty$. We get

$$
\begin{aligned}
& n_{i}^{(1)}=\frac{u_{i}^{(1)}}{\lambda}, \\
& u_{i}^{(1)}=\frac{\phi^{(1)}}{\lambda}, \\
& \phi^{(1)}=n_{i}^{(1)} .
\end{aligned}
$$

From Eq. (44)-(46) we get the phase velocity as

$$
\lambda^{2}= \pm 1
$$

Substituting the Eqs. (31)-(34) in Eqs. (38)-(40) and collecting order $O\left(\varepsilon^{5 / 2}\right)$, we get

$$
\begin{gathered}
\frac{\partial n_{i}^{(1)}}{\partial \tau}-\lambda \frac{\partial n_{i}^{(2)}}{\partial \xi}+\frac{\partial n_{i}^{(1)} u_{i}^{(1)}}{\partial \xi}+\frac{\partial u_{i}^{(2)}}{\partial \xi}=0, \\
\frac{\partial u_{i}^{(1)}}{\partial \tau}-\lambda \frac{\partial u_{i}^{(2)}}{\partial \xi}+u_{i}^{(1)} \frac{\partial u_{i}^{(1)}}{\partial \xi}=-\frac{\partial \phi^{(2)}}{\partial \xi^{2}}, \\
\frac{\partial \phi^{(1)}}{\partial \xi^{2}}=\phi^{(2)}+\frac{1}{2}\left(\phi^{(1)}\right)^{2}-n_{i}^{(1)}
\end{gathered}
$$

Differentiating Eq. (50) With respect to $\xi$ and substituting for $\frac{\partial i_{i}^{(2)}}{\partial \xi}$ from Eq. (48) and for $\frac{\partial u_{i}^{(2)}}{\partial \xi}$ from Eq. (49), we finally obtain

$$
\frac{\partial \phi^{(1)}}{\partial \tau}+\phi^{(1)} \frac{\partial \phi^{(1)}}{\partial \xi}+\frac{1}{2} \frac{\partial^{3} \phi^{(1)}}{\partial \xi^{3}}=0 .
$$

Eq. (51) is known as $\mathrm{KdV}$ equation. $\phi^{(1)} \frac{\partial \phi^{(1)}}{\partial \xi}$ is the nonlinear term and $\frac{1}{2} \frac{\partial^{3} \phi^{(1)}}{\partial \xi^{3}}$ is the dispersive terms. Only nonlinearity can impose energy into the wave and the wave breaks but in presence of both nonlinearity and dispersive a stable wave profile is possible.

The steady-state solution of this $\mathrm{KdV}$ equation is obtained by transforming the independent variables $\xi$ and $\tau$ to $\eta=\xi-u_{0} \tau$ where $u_{0}$ is a constant velocity normalized by $c_{s}$.

The steady state solution of the KdV Eq. (51) can be written as

$$
\phi^{(1)}=\phi_{m} \operatorname{sech}^{2}\left(\frac{\eta}{\Delta}\right)
$$

where $\phi_{m}=3 u_{0}$ and $\Delta$ are the amplitude and width of the solitary waves. It is clear that height, width and speed of the pulse propotional to $u_{0}, \frac{1}{\sqrt{u_{0}}}$, and $u_{0}$ respectively. As $\phi_{m}$ the amplitude is equal to $3 u_{0}$ so $u_{0}$ specify the energy of the 


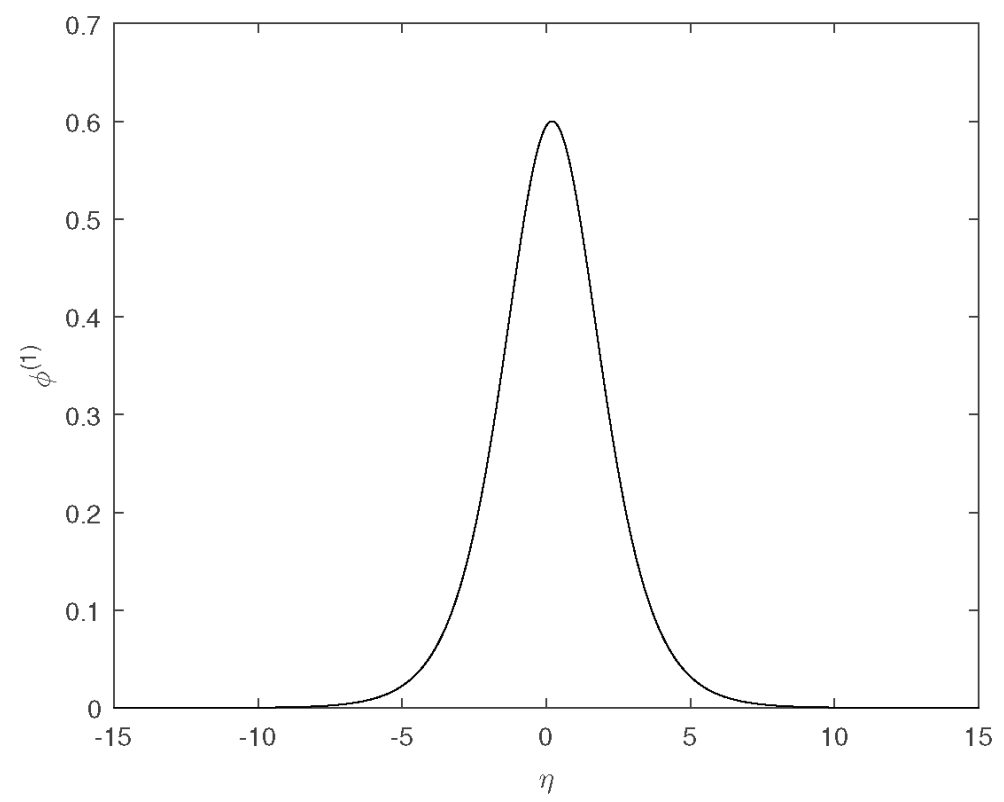

Figure 1.

Solitary wave solution of Eq. (52) for the parameter value $t=1, u_{\circ}=0.2$.

solitary waves. So the larger the energy, the greater the speed, larger the amplitude and narrower the width (Figure 1).

\section{Damped force KdV equation}

Let us consider an unmagnetized collisional dusty plasma that contains cold inertial ions, stationary dusts with negative charge and Maxwellian electrons. The normalized ion fluid equations which include the equation of continuity, equation of momentum balance and Poisson's equation, governing the DIAWs, are given by

$$
\begin{gathered}
\frac{\partial n_{i}}{\partial t}+\frac{\partial\left(n_{i} u_{i}\right)}{\partial x}=0, \\
\frac{\partial u_{i}}{\partial t}+u_{i} \frac{\partial u_{i}}{\partial x}=-\frac{\partial \phi}{\partial x}-\nu_{i d} u_{i}, \\
\frac{\partial^{2} \phi}{\partial x^{2}}=(1-\mu) n_{e}-n+\mu,
\end{gathered}
$$

where $n_{j}$ ( $j=i, e$ for ion, electron), $u_{i}, \phi$ are the number density, ion fluid velocity and the electrostatic wave potential respectively. Here $\mu=\frac{Z_{d} n_{d 0}}{n_{0}}, \nu_{i d}$ is the dust ion collisional frequency and the term $S(x, t)[4,5]$, is a charged density source arising from experimental conditions for a single definite purpose. $n_{0}, Z_{d}, n_{d 0}$ are the

\subsection{Normalization}

$$
n_{i} \rightarrow \frac{n_{i}}{n_{0}}, u_{i} \rightarrow \frac{u_{i}}{C_{s}}, \phi \rightarrow \frac{e \phi}{K_{B} T_{e}}, x \rightarrow \frac{x}{\lambda_{D}}, t \rightarrow \omega_{p i} t
$$


where $C_{s}\left(=\sqrt{\left(\frac{K_{B} T_{e}}{m_{i}}\right)}\right)$ is the ion acoustic speed, $T_{e}$ as electron temperature, $K_{B}$ as Boltzmann constant, $e$ as magnitude of electron charge and $m_{i}$ as mass of ions. $\lambda_{D}\left(=\left(\frac{T_{e}}{4 \pi n_{e 0} e^{2}}\right)^{\frac{1}{2}}\right)$ is the Debye length and $\omega_{p i}\left(=\left(\frac{4 \pi n_{e} e^{2}}{m_{i}}\right)^{\frac{1}{2}}\right)$ as ion-plasma frequency.

The normalized electron density is given by

$$
n_{e}=e^{\phi} .
$$

\subsection{Phase velocity and nonlinear evolution equation}

We introduced the same stretched coordinates use in Eq.(31). The expansion of the dependent variables also considered as (32)-(34) with

$$
\begin{gathered}
\nu_{i d} \sim \varepsilon^{3 / 2} \nu_{i d 0} . \\
S \sim \varepsilon^{2} S_{2} .
\end{gathered}
$$

Substituting (31)-(34) and (58)-(59) along with stretching coordinates into Eqs. (53)-(55) and equating the coefficients of lowest order of $\varepsilon$, we get the phase velocity as

$$
\lambda=\frac{1}{\sqrt{(1-\mu)}} .
$$

Taking the coefficients of next higher order of $\varepsilon$, we obtain the damped force $\mathrm{KdV}$ equation

$$
\frac{\partial \phi^{(1)}}{\partial \tau}+A \phi^{(1)} \frac{\partial \phi^{(1)}}{\partial \xi}+B \frac{\partial^{3} \phi^{(1)}}{\partial \xi^{3}}+C \phi^{(1)}=B \frac{\partial S_{2}}{\partial \xi},
$$

where $A=\frac{3-\lambda^{2}}{2 \lambda}, B=\frac{\lambda^{3}}{2}, C=\frac{\nu_{i d 0}}{2}$.

It has been noticed that the behavior of nonlinear waves changes significantly in the presence of external periodic force. It is paramount to note that the source term or forcing term due to the presence of space debris in plasmas may be of different kind, for example, Gaussian forcing term [4], hyperbolic forcing term [4], (in the form of $\operatorname{sech}^{2}(\xi, \tau)$ and $\operatorname{sech}^{4}(\xi, \tau)$ functions) and trigonometric forcing term [6] (in the form of $\sin (\xi, \tau)$ and $\cos (\xi, \tau)$ functions). Motivated by these work we assume that $S_{2}$ is a linear function of $\xi$ such as $S_{2}=\frac{f_{0} \xi}{B} \cos (\omega \tau)+P$, where P is some constant and $f_{0}, \omega$ denote the strength and the frequency of the source respectively. Put the expression of $S_{2}$ in Eq. (61) we get,

$$
\frac{\partial \phi^{(1)}}{\partial \tau}+A \phi^{(1)} \frac{\partial \phi^{(1)}}{\partial \xi}+B \frac{\partial^{3} \phi^{(1)}}{\partial \xi^{3}}+C \phi^{(1)} 1=f_{0} \cos (\omega \tau),
$$

which is termed as damped and forced $\mathrm{KdV}$ (DFKdV) equation.

In absence of $C$ and $f_{0}$, i.e., for $C=0$ and $f_{0}=0$ the Eq.(62) takes the form of well-known $\mathrm{KdV}$ equation with the solitary wave solution

$$
\phi_{1}=\phi_{m} \operatorname{sech}^{2}\left(\frac{\xi-M \tau}{W}\right)
$$


where $\phi_{m}=\frac{3 M}{A}$ and $W=2 \sqrt{\frac{B}{M}}$, with $M$ as the Mach number.

In this case, it is well established that

$$
I=\int_{-\infty}^{\infty} \phi_{1}^{2} d \xi
$$

is a conserved. For small values of $C$ and $f_{0}$, let us assume that the solution of Eq. (62) is of the form

$$
\phi_{1}=\phi_{m}(\tau) \operatorname{sech}^{2}\left(\frac{x-M(\tau) \tau}{W(\tau)}\right)
$$

where $M(\tau)$ is an unknown function of $\tau$ and $\phi_{m}(\tau)=\frac{3 M(\tau)}{A}, W(\tau)=2 \sqrt{B / M(\tau)}$. Differentiating Eq. (64) with respect to $\tau$ and using Eq. (62), one can obtain

$$
\begin{aligned}
& \frac{d I}{d \tau}+2 C I=2 f_{0} \cos (\omega \tau) \int_{-\infty}^{\infty} \phi_{1} d \xi \\
& \Rightarrow \frac{d I}{d \tau}+2 C I=\frac{24 f_{0} \sqrt{B}}{A} \sqrt{M(\tau)} \cos (\omega \tau) .
\end{aligned}
$$

Again,

$$
\begin{gathered}
I=\int_{-\infty}^{\infty} \phi_{1}^{2} d \xi \\
I=\int_{-\infty}^{\infty} \phi_{m}^{2}(\tau) \operatorname{sech}^{4}\left(\frac{\xi-M(\tau) \tau}{W(\tau)}\right) d \xi \\
I=\frac{24 \sqrt{B}}{A^{2}} M^{3 / 2}(\tau) .
\end{gathered}
$$

Using Eq. (66) and (67) the expression of $M(\tau)$ is obtained as

$$
M(\tau)=\left(M-\frac{8 A C f_{0}}{16 C^{2}+9 \omega^{2}}\right) e^{-\frac{4}{3} C \tau}+\frac{6 A f_{0}}{16 C^{2}+9 \omega^{2}}\left(\frac{4}{3} C \cos (\omega \tau)+\omega \sin (\omega \tau)\right) .
$$

Therefore, the solution of the Eq. (62) is

$$
\phi_{1}=\phi_{m}(\tau) \operatorname{sech}^{2}\left(\frac{\xi-M(\tau) \tau}{W(\tau)}\right)
$$

where $\phi_{m}(\tau)=\frac{3 M(\tau)}{A}$ and $W(\tau)=2 \sqrt{\frac{B}{M(\tau)}}$. The effect of the parameters, i.e., ion collision frequency parameter $\left(\nu_{i d 0}\right)$, strength of the external force $\left(f_{0}\right)$ on the solitary wave solution of the damp force KdV Eq. (62) have been numerically studied. In Figure 2, the soliton solution of (62) is plotted from (63)in the absence of external periodic force and damping.

In Figure 3, the soliton solution of the damp force $\mathrm{KdV}$ equation is plotted from Eq. (65) for different values of the strength of the external periodic force $\left(f_{0}\right)$. The values of other parameters are $M_{0}=0.2, \omega=1, \tau=1, \mu=0.2, \nu_{i d 0}=0.01$. It is observed that the solution produces solitary waves and the amplitude of the solitary waves increases as the value of the parameter $f_{0}$ increases. In Figure 4, damp force $\mathrm{KdV}$ equation is plotted from Eq. (65) for different values of the dust ion collision 


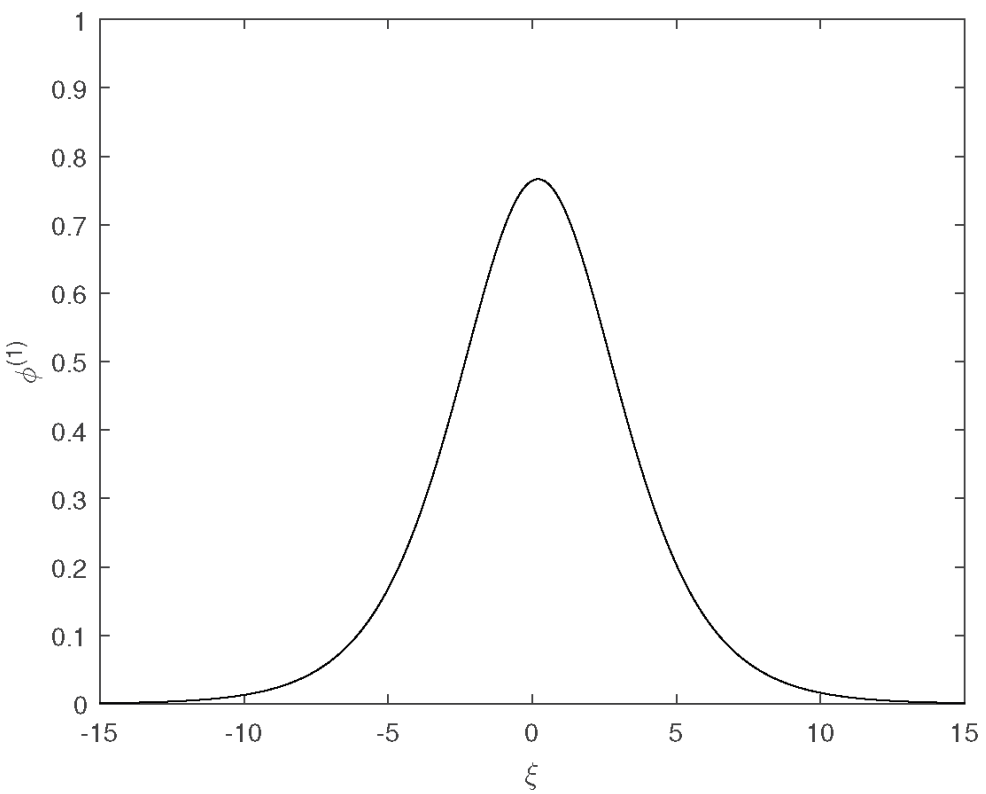

Figure 2.

Solitary wave solution of Eq. (62) in the absence of damping $\left(\nu_{i d o}=0\right)$ and external force $\left(f_{\circ}=0\right)$ with the parameter value $M_{\circ}=0.2, \omega=1, \tau=1, \mu=0.2$.

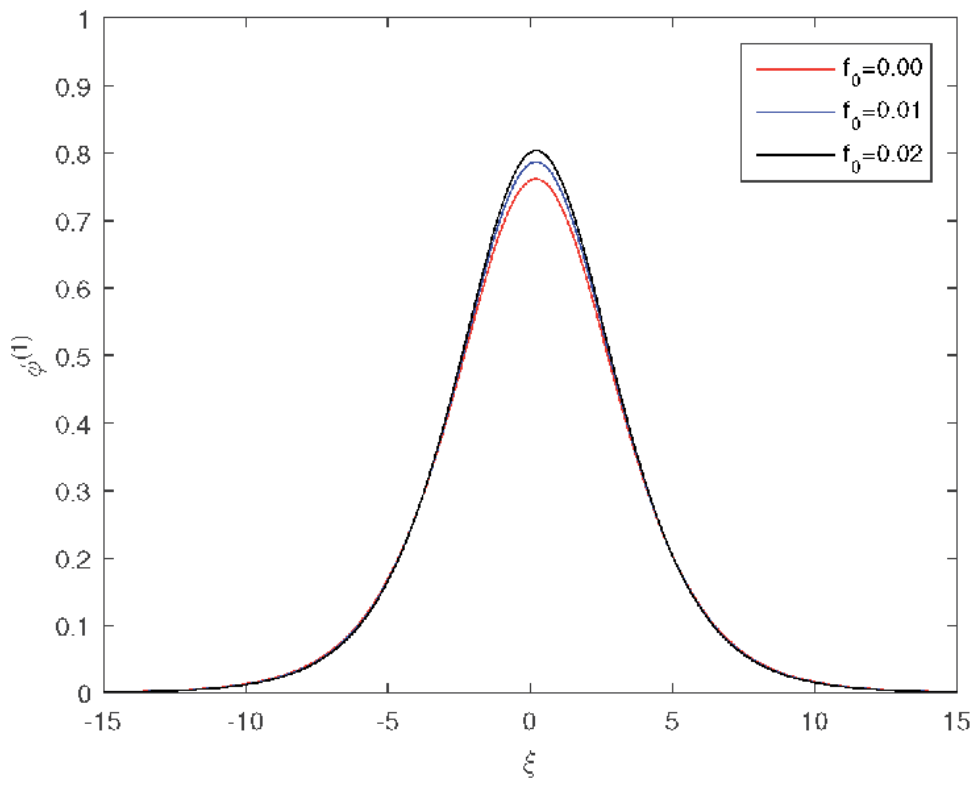

Figure 3 .

Variation of solitary wave from Eq. (62) for the different values of $f_{\circ}$ with $M_{\circ}=0.2, \omega=1, \tau=1, \mu=0.2$, $\nu_{i d o}=0.01$.

frequency parameter $\left(\nu_{i d 0}\right)$. The values of other parameters are $M_{0}=0.2, \omega=1$, $\tau=1, \mu=0.2, f_{0}=0.01$. It is observed that the solution produces solitary waves and the amplitude of the solitary waves decreases as the value of the parameter $\nu_{i d 0}$ increases and width of the solitary waves increases for increasing value of $\nu_{i d 0}$. 


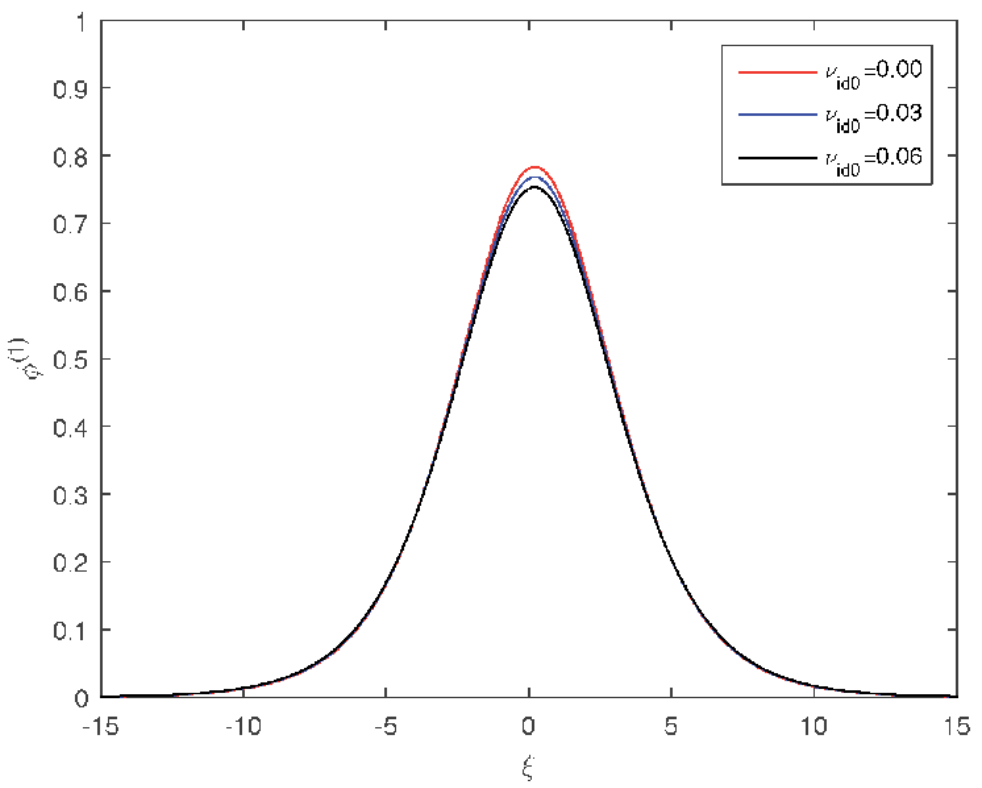

Figure 4.

Variation of solitary wave from Eq. (62) for the different values of collisional frequency $\nu_{i d o}$ with $M_{\circ}=0.2$, $\omega=1, \tau=1, \mu=0.2, f_{0}=0.01$.

\section{Damped KdV Burgers equation}

To obtain damped KdV Burgers equation we considered an unmagnetized collisional dusty plasma which contains cold inertial ions, stationary dusts with negative charge and Maxwellian distributed electrons. The normalized ion fluid equations are as follows

$$
\begin{gathered}
\frac{\partial n_{i}}{\partial t}+\frac{\partial\left(n_{i} u_{i}\right)}{\partial x}=0 \\
\frac{\partial u_{i}}{\partial t}+u_{i} \frac{\partial u_{i}}{\partial x}=-\frac{\partial \phi}{\partial x}+\eta \frac{\partial^{2} u_{i}}{\partial x^{2}}-\nu_{i d} u_{i} \\
\frac{\partial^{2} \phi}{\partial x^{2}}=(1-\mu) n_{e}-n_{i}+\mu, \\
n_{e}=e^{\phi}
\end{gathered}
$$

where $n_{i}, n_{e}, u_{i}, \phi$, are the number density of ions, the number density of electrons, the ion fluid velocity and the electrostatic wave potential, respectively.

Here normalization is taken as follows

$$
n_{i} \rightarrow \frac{n_{i}}{n_{0}}, u_{i} \rightarrow \frac{u_{i}}{C_{s}}, \phi \rightarrow \frac{e \phi}{K_{B} T_{e}}, x \rightarrow \frac{x}{\lambda_{D}}, t \rightarrow \omega_{p i} t
$$

$C_{s}\left(=\sqrt{\left(\frac{K_{B} T_{e}}{m_{i}}\right)}\right)$ is the ion acoustic speed, $T_{e}$ as electron temperature, $K_{B}$ as Boltzmann constant and $m_{i}$ as mass of ions, $e$ as magnitude of electron charge. $\lambda_{D}\left(=\left(\frac{T_{e}}{4 \pi n_{e 0} e^{2}}\right)^{\frac{1}{2}}\right)$ is the Debye length and $\omega_{p i}\left(=\left(\frac{m_{i}}{4 \pi n_{e 0} e^{2}}\right)^{\frac{1}{2}}\right)$ as ion-plasma 
frequency. Here, $\nu_{i d}$ is the dust-ion collisional frequency and $\mu=\frac{n_{0 e}}{n_{0 i}}$, where $n_{0 e}$ and $n_{0 i}$ are the unperturbed number densities of electrons and ions, respectively.

\subsection{Perturbation}

To obtain damped KdV burger we introduced the same stretched coordinates use in Eq.(31). The expansion of the dependent variables are also considered same as (32)-(34) with

$$
\begin{gathered}
\eta=\varepsilon^{1 / 2} \eta_{0}, \\
\nu_{i d} \sim \varepsilon^{3 / 2} \nu_{i d 0} .
\end{gathered}
$$

\subsection{Phase velocity and nonlinear evolution equation}

Substituting the above expansions (32)-(34) and (73)-(74) along with stretching coordinates (31) into Eqs. (69)-(71) and equating the coefficients of lowest order of $\varepsilon$, the phase velocity is obtained as

$$
\lambda=\frac{1}{\sqrt{(1-\mu)}} .
$$

Taking the coefficients of next higher order of $\varepsilon$, we obtain the DKdVB equation

$$
\frac{\partial \phi^{(1)}}{\partial \tau}+A \phi^{(1)} \frac{\partial \phi^{(1)}}{\partial \xi}+B \frac{\partial^{3} \phi^{(1)}}{\partial \xi^{3}}+C \frac{\partial^{2} \phi^{(1)}}{\partial \xi^{2}}+D \phi^{(1)}=0,
$$

where $A=\frac{3-\lambda^{2}}{2 \lambda}, B=\frac{v^{3}}{2}, C=-\frac{\eta_{10}}{2}$ and $D=\frac{\nu_{i d 0}}{2}$.

In absence of $C$ and $D$, i.e., for $C=0$ and $D=0$ the Eq.(76) takes the form of well-known $\mathrm{KdV}$ equation with the solitary wave solution

$$
\phi_{1}=\phi_{m} \operatorname{sech}^{2}\left(\frac{\xi-M_{0} \tau}{W}\right)
$$

where amplitude of the solitary waves $\phi_{m}=\frac{3 M_{0}}{A}$ and width of the solitary waves $W=2 \sqrt{\frac{B}{M_{0}}}$, with $M_{0}$ is the speed of the ion-acoustic solitary waves or Mach number.

It is well established for the $\mathrm{KdV}$ equation that,

$$
I=\int_{-\infty}^{\infty} \phi_{1}^{2} d \xi
$$

is a conserved quantity [7].

For small values of $C$ and $D$, let us assume that amplitude, width and velocity of the dust ion acoustic waves are dependent on $\tau$ and the slow time dependent solution of Eq. (76) is of the form

$$
\phi^{(1)}=\phi_{m}(\tau) \operatorname{sech}^{2}\left(\frac{\xi-M(\tau) \tau}{W(\tau)}\right)
$$

where the amplitude $\phi_{m}(\tau)=\frac{3 M(\tau)}{A}$, width $W(\tau)=2 \sqrt{B / M(\tau)}$ and velocity $M(\tau)$ have to be determined. 
Differentiating Eq. (78) with respect to $\tau$ and using Eq. (76), one can obtain

$$
\begin{aligned}
& \frac{d I}{d \tau}+2 D I=2 C \int_{-\infty}^{\infty}\left(\frac{\partial \phi_{1}}{\partial \xi}\right)^{2} d \xi \\
& \Rightarrow \frac{d I}{d \tau}+2 D I=2 C \times \frac{24}{5} \frac{M^{5 / 2}(\tau)}{A^{2} \sqrt{B}} .
\end{aligned}
$$

where,

$$
\int_{-\infty}^{\infty}\left(\frac{\partial \phi_{1}}{\partial \xi}\right)^{2} d \xi=\frac{24}{5} \frac{M^{5 / 2}(\tau)}{A^{2} \sqrt{B}}
$$

and

$$
\begin{gathered}
I=\int_{-\infty}^{\infty} \phi_{1}^{2} d \xi \\
I=\int_{-\infty}^{\infty} \phi_{m}^{2}(\tau) \operatorname{sech}^{4}\left(\frac{\xi-M(\tau) \tau}{W(\tau)}\right) d \xi \\
I=\frac{24 \sqrt{B}}{A^{2}} M^{3 / 2}(\tau) .
\end{gathered}
$$

Substituting Eq. (81) and (82) into Eq. (80), we obtain

$$
\frac{d M(\tau)}{d \tau}+P M(\tau)=Q M^{2}(\tau)
$$

which is the Bernoulli's equation, where $P=\frac{4}{3} D$ and $Q=\frac{4}{15} \frac{C}{B}$.

The solution of the Eq. (83) is

$$
M(\tau)=\frac{P M_{0}}{M_{0} Q\left(1-e^{P \tau}\right)+P e^{P \tau}}
$$

Therefore, the slow time dependence form of the ion acoustic solitary wave solution of the DKdVB Eq. (76) is given by (79)where.

$$
M(\tau)=\frac{P M_{0}}{M_{0} Q\left(1-e^{P \tau}\right)+P e^{P \tau}} \text { and } M(0)=M_{0} \text { for } \tau=0 .
$$

\section{Damped force MKdV equation}

Let us consider an unmagnetized collisional dusty plasma that contains cold inertial ions, stationary dusts with negative charge and Maxwellian distributed electrons. The normalized ion fluid equations which include the equation of continuity, equation of momentum balance and Poisson's equation, governing the DIAWs, are given by

$$
\begin{gathered}
\frac{\partial n_{i}}{\partial t}+\frac{\partial\left(n_{i} u_{i}\right)}{\partial x}=0 \\
\frac{\partial u_{i}}{\partial t}+u_{i} \frac{\partial u_{i}}{\partial x}=-\frac{\partial \phi}{\partial x}-\nu_{i d} u \\
\frac{\partial^{2} \phi}{\partial x^{2}}=(1-\mu) n_{e}-n_{i}+\mu+S(x, t)
\end{gathered}
$$


where $n_{j}$ ( $j=i$,e for ion, electron), $u_{i}, \phi$ are the number density, ion fluid velocity and the electrostatic wave potential respectively. Here $\mu=\frac{Z_{d} n_{d 0}}{n_{0}}, \nu_{i d}$ is the dust-ion collisional frequency and the term $S(x, t)[4,5]$, is a charged density source arising from experimental conditions for a single definite purpose. $n_{0}, Z_{d}, n_{d 0}$ are the normalization:

$$
n_{i} \rightarrow \frac{n_{i}}{n_{0}}, u_{i} \rightarrow \frac{u_{i}}{C_{s}}, \phi \rightarrow \frac{e \phi}{K_{B} T_{e}}, x \rightarrow \frac{x}{\lambda_{D}}, t \rightarrow \omega_{p i} t
$$

where $C_{s}\left(=\sqrt{\left(\frac{K_{B} T_{e}}{m_{i}}\right)}\right)$ is the ion acoustic speed, $T_{e}$ as electron temperature, $K_{B}$ as Boltzmann constant, $e$ as magnitude of electron charge and $m_{i}$ as mass of ions. $\lambda_{D}\left(=\left(\frac{T_{e}}{4 \pi n_{e 0} e^{2}}\right)^{\frac{1}{2}}\right)$ is the Debye length and $\omega_{p i}\left(=\left(\frac{4 \pi n_{e} e^{2}}{m_{i}}\right)^{\frac{1}{2}}\right)$ as ion-plasma frequency.

The normalized $q$-nonextensive electron number density takes the form [8]:

$$
n_{e}=n_{e 0}\{1+(q-1) \phi\}^{\frac{q+1}{2(q-1)}}
$$

\section{Phase velocity and nonlinear evolution equation}

We introduced the same stretched coordinates use in Eq. (31). The expansion of the dependent variables also considered same as (32)-(34) and (58)-(59). Substituting (31)-(34) and (58)-(59) along with stretching coordinates into Eqs. (84)-(86) and equating the coefficients of lowest order of $\varepsilon$, we get the phase velocity as

$$
\lambda=\frac{1}{\sqrt{a(1-\mu)}},
$$

with $a=\frac{q+1}{2}$. Now taking the coefficients of next higher order of $\varepsilon$ [i.e., coefficient of $\varepsilon^{5 / 2}$ from Eqs. (84) and (85) and coefficient of $\varepsilon^{2}$ from Eq. (86)], we obtain the DFKdV equation

$$
\frac{\partial \phi^{(1)}}{\partial \tau}+A \phi^{(1)} \frac{\partial \phi^{(1)}}{\partial \xi}+B \frac{\partial^{3} \phi^{(1)}}{\partial \xi^{3}}+C \phi^{(1)}=B \frac{\partial S_{2}}{\partial \xi},
$$

where $A=\left(\frac{3}{2 \lambda}-\frac{b \lambda}{a}\right), B=\frac{\lambda^{3}}{2}$ and $C=\frac{\nu_{i d 0}}{2}$, with $b=\frac{(q+1)(3-q)}{8}$.

Now at the certain values, for example $q=0.6$ and $\mu=0.5$, there is a critical point at which $A=0$, which imply the infinite growth of the amplitude of the DIASW solution as nonlinearity goes to zero. Therefore, at the critical point at which $A=0$ the stretching (31) is not valid. For describing the evolution of the nonlinear system at or near the critical point we introduce the new stretched coordinate as

$$
\xi=\varepsilon(x-\lambda t), \tau=\varepsilon^{3} t,
$$

and expand of the dependent variables same as Eqs. (32)-(34) with

$$
\begin{aligned}
\nu_{i d} & \sim \varepsilon^{3} \nu_{i d 0}, \\
S & \sim \varepsilon^{3} S_{2} .
\end{aligned}
$$


Now substituting Eq. (32)-(34) and (91)-(93) into the basic Eqs. (84)-(86) and equating the coefficients of lowest order of $\varepsilon$, [i.e., coefficients of $\varepsilon^{2}$ from Eq. (84) and (85) and coefficients of $\varepsilon$ from Eq. (86)], we obtain the following relations:

$$
\begin{gathered}
n_{i}^{(1)}=\frac{u_{i}^{(1)}}{\lambda}, \\
u_{i}^{(1)}=\frac{\phi^{(1)}}{\lambda}, \\
n_{i}^{(1)}=a(1-\mu) \phi^{(1)} .
\end{gathered}
$$

Equating the coefficients of next higher order of $\varepsilon$, [i.e., coefficients of $\varepsilon^{3}$ from Eq. (84) and (85) and coefficients of $\varepsilon$ from Eq. (86)], we obtain the following relations:

$$
\begin{gathered}
n_{i}^{(2)}=\frac{1}{\lambda}\left(u_{i}^{(2)}+n_{i}^{(1)} u_{i}^{(1)}\right) \\
\frac{\partial u_{i}^{(1)}}{\partial \xi}=\frac{1}{\lambda}\left(u_{i}^{(1)} \frac{\partial u_{i}^{(1)}}{\partial \xi}+\frac{\partial \phi^{(2)}}{\partial \xi}\right) \\
n_{i}^{(2)}=a(1-\mu)\left(a \phi^{(2)}+b\left(\phi^{(1)}\right)^{2}\right)
\end{gathered}
$$

Equating the coefficients of next higher order of $\varepsilon$, [i.e., coefficients of $\varepsilon^{4}$ from Eq. (84) and (85) and coefficients of $\varepsilon$ from Eq. (86)], we obtain the following relations:

$$
\begin{gathered}
\frac{\partial n_{i}^{(1)}}{\partial \tau}-\lambda \frac{\partial n_{i}^{(3)}}{\partial \xi}+\frac{\partial u_{i}^{(3)}}{\partial \xi}+\frac{\partial\left(n_{i}^{(1)} u_{i}^{(2)}\right)}{\partial \xi}+\frac{\partial\left(n_{i}^{(2)} u_{i}^{(1)}\right)}{\partial \xi}=0 \\
\frac{\partial u_{i}^{(1)}}{\partial \tau}-\lambda \frac{\partial u_{i}^{(3)}}{\partial \xi}+\frac{\partial \phi^{(3)}}{\partial \xi}+\frac{\partial\left(u_{i}^{(1)} u_{i}^{(2)}\right)}{\partial \xi}+\nu_{i d 0} u_{i}^{(1)}=0 \\
\frac{\partial^{2} \phi^{(1)}}{\partial \xi^{2}}=(1-\mu)\left(a \phi^{(3)}+2 b \phi^{(1)} \phi^{(2)}+c\left(\phi^{(1)}\right)^{3}\right)-n_{i}^{(3)}+S_{2}
\end{gathered}
$$

where $a=\frac{(1+q)}{2}, b=\frac{(1+q)(3-q)}{8}$ and $c=\frac{(1+q)(3-q)(5-3 q)}{48}$.

From Eq. (94)-(96), one can obtain the Phase velocity as $\lambda^{2}=\frac{1}{a(1-\mu)}$ and from Eqs. (94)-(102), one can obtain the following nonlinear evaluation equation as:

$$
\frac{\partial \phi^{(1)}}{\partial \tau}+A_{1}\left(\phi^{(1)}\right)^{2} \frac{\partial \phi^{(1)}}{\partial \xi}+B_{1} \frac{\partial^{3} \phi^{(1)}}{\partial \xi^{3}}+C_{1} \phi^{(1)}=B_{1} \frac{\partial S_{2}}{\partial \xi},
$$

where $A_{1}=\frac{15}{4 \lambda^{3}}-\frac{3 \lambda^{3} c(1-\mu)}{2}, B_{1}=\frac{\lambda^{3}}{2}$ and $C_{1}=\frac{\nu_{i d} 0}{2}$.

It has been noticed that the behavior of nonlinear waves changes significantly in the presence of external periodic force. For simplicity, we assume that $S_{2}$ is a linear function of $\xi$ such as $S_{2}=f_{0} \xi \cos (\omega \tau)+P$, where $\mathrm{P}$ is some constant and $f_{0}, \omega$ denote the strength and the frequency of the source respectively. Put the expression of $S_{2}$ in the Eq. (103) we get, 


$$
\frac{\partial \phi^{(1)}}{\partial \tau}+A_{1}\left(\phi^{(1)}\right)^{2} \frac{\partial \phi^{(1)}}{\partial \xi}+B_{1} \frac{\partial^{3} \phi^{(1)}}{\partial \xi^{3}}+C_{1} \phi^{(1)}=B_{1} f_{0} \cos (\omega \tau) .
$$

Such a form of this source function is observed in experimental situations or conditions for a particular device. Eq. (104) is termed as damped force modified Korteweg-de Varies (DFMKdV) equation.

In absence of $C_{1}$ and $f_{0}$, i.e., for $C_{1}=0$ and $f_{0}=0$ the Eq.(104) takes the form of well-known MKdV equation.

The slow time dependence form of the ion acoustic waves solution of the DFMKdV Eq. (104) is given by,

$$
\phi^{(1)}=\phi_{m}(\tau) \operatorname{sech}\left(\frac{\xi-M(\tau) \tau}{W(\tau)}\right)
$$

where $M(\tau)$ is given by equation

$$
\begin{aligned}
M(\tau)= & {\left[\frac{\pi f_{0} B_{1} \sqrt{A_{1} / 6}}{2}\left(\frac{\omega}{\omega^{2}+4 C_{1}^{2}}\right)\left\{\sin (\omega \tau)+\frac{2 C_{1}}{\omega} \cos (\omega \tau)\right\}\right.} \\
& \left.+\left\{\sqrt{M}-\pi f_{0} B_{1} \sqrt{A_{1} / 24}\left(\frac{2 C_{1}}{\omega^{2}+4 C_{1}^{2}}\right)\right\} e^{-\nu_{i d 0} \tau}\right]^{2} .
\end{aligned}
$$

The amplitude and width are as follows:

$$
\begin{gathered}
\phi_{m}(\tau)=\frac{1}{\sqrt{A}}\left(\sqrt { 6 } \left[\frac{\pi f_{0} B_{1} \sqrt{A_{1} / 6}}{2}\left(\frac{\omega}{\omega^{2}+4 C_{1}^{2}}\right)\left\{\sin (\omega \tau)+\frac{2 C_{1}}{\omega} \cos (\omega \tau)\right\}\right.\right. \\
\left.\left.+\left\{\sqrt{M}-\pi f_{0} B_{1} \sqrt{A_{1} / 24}\left(\frac{2 C_{1}}{\omega^{2}+4 C_{1}^{2}}\right)\right\} e^{-\nu_{i d 0} \tau}\right]\right) \\
W(\tau)=\frac{\sqrt{B_{1}}}{W_{1}+W_{2}}
\end{gathered}
$$

where

$$
\begin{aligned}
& W_{1}=\frac{\pi f_{0} B_{1} \sqrt{A_{1} / 6}}{2}\left(\frac{\omega}{\omega^{2}+4 C_{1}^{2}}\right)\left\{\sin (\omega \tau)+\frac{2 C_{1}}{\omega} \cos (\omega \tau)\right\} \\
& W_{2}=\left\{\sqrt{M}-\pi f_{0} B_{1} \sqrt{A_{1} / 24}\left(\frac{2 C_{1}}{\omega^{2}+4 C_{1}^{2}}\right)\right\} e^{-\nu_{i d 0} \tau}
\end{aligned}
$$

\section{Damped force Zakharov-Kuznetsov equation}

Let us consider a plasma model [9] consisting of cold ions, Maxwellian electrons in the presence of dust particles and the external static magnetic field $B=\hat{y} B_{0}$ along the $y$-axis. The normalized continuity, momentum and Poisson's equations are as follows

$$
\frac{\partial n}{\partial t}+\frac{\partial(n u)}{\partial x}+\frac{\partial(n v)}{\partial y}+\frac{\partial(n w)}{\partial z}=0
$$




$$
\begin{gathered}
\frac{\partial u}{\partial t}+\left(u \frac{\partial}{\partial x}+v \frac{\partial}{\partial y}+w \frac{\partial}{\partial z}\right) u=-\frac{\partial \phi}{\partial x}-\frac{\Omega_{i}}{\omega_{p i}} w \\
\frac{\partial v}{\partial t}+\left(u \frac{\partial}{\partial x}+v \frac{\partial}{\partial y}+w \frac{\partial}{\partial z}\right) v=-\frac{\partial \phi}{\partial y}-\nu_{i d} v \\
\frac{\partial w}{\partial t}+\left(u \frac{\partial}{\partial x}+v \frac{\partial}{\partial y}+w \frac{\partial}{\partial z}\right) w=-\frac{\partial \phi}{\partial z}+\frac{\Omega_{i}}{\omega_{p i}} u \\
\frac{\partial^{2} \phi}{\partial x^{2}}+\frac{\partial^{2} \phi}{\partial y^{2}}+\frac{\partial^{2} \phi}{\partial z^{2}}=\delta_{1}+\delta_{2} n_{e}-n
\end{gathered}
$$

The normalized electron density is given by

$$
n_{e}=e^{\phi},
$$

where $n, n_{e}, u_{i}(=u, v, w), T_{e}, m_{i}, e, \phi, \Omega_{i}, \omega_{p i}$, vid and $\lambda_{D}$ are the ion number density, electron number density, ion velocity, electron temperature, ion mass, electron charge, electrostatic potential, ion cyclotron frequency, ion plasma frequency, dust ion collision frequency and Debye length respectively.

Here the normalization is done as follows:

$$
n \rightarrow \frac{n}{n_{0}}, n_{e} \rightarrow \frac{n_{e}}{n_{e 0}}, u_{i} \rightarrow \frac{u_{i}}{C_{s}}, \phi \rightarrow \frac{e \phi}{T_{e}}, x \rightarrow \frac{x}{\lambda_{D}}, t \rightarrow \omega_{p i} t
$$

Here $\delta_{1}=\frac{n_{d 0}}{n_{i 0}}, \delta_{2}=\frac{n_{e 0}}{n_{i 0}}$ with the condition $\delta_{1}+\delta_{2}=1 . \lambda_{D}=\left(\frac{T_{e}}{4 \pi n_{e 0} e^{2}}\right)^{1 / 2}$, $\omega_{p i}^{-1}=\left(\frac{m_{i}}{4 \pi n_{e} e^{2}}\right)^{1 / 2}, C_{s}=\sqrt{\frac{T_{e}}{m_{i}}}$.

To obtain the DFZK equation we introduce the new stretched coordinates as

$$
\begin{gathered}
\xi=\varepsilon^{1 / 2} x \\
\zeta=\varepsilon^{1 / 2}(x-\lambda t), \\
\eta=\varepsilon^{1 / 2} y, \\
\tau=\varepsilon^{3 / 2} t
\end{gathered}
$$

where $\varepsilon$ is the strength of nonlinearity and $\lambda$ is the phase velocity of waves. The expression of the dependent variables as follows:

$$
\begin{gathered}
n=1+\varepsilon n_{1}+\varepsilon^{2} n_{2}+\cdots \\
u=0+\varepsilon^{3 / 2} u_{1}+\varepsilon^{2} u_{2}+\cdots \\
v=0+\varepsilon v_{1}+\varepsilon^{2} v_{2}+\cdots \\
w=0+\varepsilon^{3 / 2} w_{1}+\varepsilon^{2} w_{2}+\cdots \\
\phi=0+\varepsilon \phi_{1}+\varepsilon^{2} \phi_{2}+\cdots \\
\nu_{i d} \sim \varepsilon^{3 / 2} \nu_{i d 0} \\
S(x, y, z) \sim \varepsilon^{2} S_{2}(x, y, z)
\end{gathered}
$$

Substituting the equations (112)-(119) into the system of Eqs. (106)-(110) equating the coefficient of $\varepsilon$, we get 


$$
\phi_{1}=\frac{n_{1}}{\delta_{2}}
$$

Equating the coefficient of $\varepsilon^{3 / 2}$, we get

$$
\begin{gathered}
n_{1}=\frac{v_{1}}{\lambda} \\
w_{1}=-\frac{\omega_{p i}}{\Omega_{i}} \frac{\partial \phi_{1}}{\partial \xi}, \\
v_{1}=\frac{\phi_{1}}{\lambda}, \\
u_{1}=\frac{\omega_{p i}}{\Omega_{i}} \frac{\partial \phi_{1}}{\partial \eta} .
\end{gathered}
$$

Considering the coefficient of $\varepsilon^{2}$, the following relationships are obtained

$$
\begin{aligned}
w_{2} & =\lambda \frac{\omega_{p i}}{\Omega_{i}} \frac{\partial u_{1}}{\partial \zeta}, \\
u_{2} & =-\lambda \frac{\omega_{p i}}{\Omega_{i}} \frac{\partial w_{1}}{\partial \zeta}, \\
\frac{\partial^{2} \phi_{1}}{\partial \xi^{2}}+\frac{\partial^{2} \phi_{1}}{\partial \zeta^{2}}+\frac{\partial^{2} \phi_{1}}{\partial \eta^{2}} & =\delta_{1}\left(1-\phi_{2}+\frac{\phi_{1}^{2}}{2}\right)-n_{2}+S_{2} .
\end{aligned}
$$

Comparing the coefficients of $\varepsilon^{5 / 2}$, we obtain

$$
\begin{gathered}
\frac{\partial n_{1}}{\partial \tau}-\lambda \frac{\partial n_{2}}{\partial \zeta}+\frac{\partial u_{2}}{\partial \xi}+\frac{\partial}{\partial \zeta}\left(n_{1} v_{1}\right)+\frac{\partial v_{2}}{\partial \zeta}+\frac{\partial w_{2}}{\partial \eta}=0 \\
\frac{\partial v_{1}}{\partial \tau}-\lambda \frac{\partial v_{2}}{\partial \zeta}+v_{1} \frac{\partial v_{1}}{\partial \zeta}+\frac{\partial \phi_{2}}{\partial \zeta}-\nu_{i d 0} v_{1}=0 .
\end{gathered}
$$

Using the relationships (120)-(124), one can obtain the linear dispersion relation as

$$
1-\lambda^{2} \delta_{2}=0
$$

Expressing all the perturbed quantities in terms of $\phi_{1}$ from Eq. (125)-(129), the damped forced ZK equation is obtained as

$$
\frac{\partial \phi_{1}}{\partial \tau}+A \phi_{1} \frac{\partial \phi_{1}}{\partial \zeta}+B \frac{\partial^{3} \phi_{1}}{\partial \zeta^{3}}+D \phi_{1}+C \frac{\partial}{\partial \zeta}\left(\frac{\partial^{2} \phi_{1}}{\partial \xi^{2}}+\frac{\partial^{2} \phi_{1}}{\partial \eta^{2}}\right)+B \frac{\partial S_{2}}{\partial \zeta}=0
$$

where

$$
A=\frac{3}{2 \lambda}-\frac{\lambda}{2}, B=\frac{\lambda}{2 \delta_{2}}, C=\frac{\lambda}{2 \delta_{2}}\left(1+\frac{\omega_{p i}^{2}}{\Omega_{i}^{2}}\right), \quad D=\frac{\nu_{i d 0}}{2} .
$$

Choudhury et al. [5] studied analytical electron acoustic solitary wave (EASW) solution in the presence of periodic force for an unmagnetized plasma consisting of cold electron fluid, superthermal hot electrons and stationary ions. Motivated by the these works, here we consider the source term as $S_{2}=\frac{f_{0}}{B}(e \zeta+f \xi+g \eta) \cos (\omega \tau)$, 
where $f_{0}$ and $\omega$ denote the strength and frequency of the source term respectively. Then Eq. (131) is of the form,

$$
\frac{\partial \phi_{1}}{\partial \tau}+A \phi_{1} \frac{\partial \phi_{1}}{\partial \zeta}+B \frac{\partial^{3} \phi_{1}}{\partial \zeta^{3}}+D \phi_{1}+C \frac{\partial}{\partial \zeta}\left(\frac{\partial^{2} \phi_{1}}{\partial \xi^{2}}+\frac{\partial^{2} \phi_{1}}{\partial \eta^{2}}\right)=F_{0} \cos (\omega \tau)
$$

where $F_{0}=-\frac{e f_{0}}{B}$. To find the analytical solution of Eq. (132), we transform the damped-forced $\mathrm{ZK}$ equation to the $\mathrm{KdV}$ equation. We introduce new variable:

$$
\xi=(l \zeta+m \xi+n \eta)
$$

where $l, m, n$ are the direction cosines of the line of wave propagation, with $l^{2}+m^{2}+n^{2}=1$. Substituting Eqs. (133) into the Eq. (132), we get

$$
\begin{gathered}
\frac{\partial \phi_{1}}{\partial \tau}+A l \phi_{1} \frac{\partial \phi_{1}}{\partial \xi}+B l^{3} \frac{\partial^{3} \phi_{1}}{\partial \xi^{3}}+C l\left(m^{2}+n^{2}\right) \frac{\partial^{3} \phi_{1}}{\partial \xi^{3}}+D \phi_{1}=F_{0} \cos (\omega \tau) \\
\Rightarrow \frac{\partial \phi_{1}}{\partial \tau}+P \phi_{1} \frac{\partial \phi_{1}}{\partial \xi}+Q \frac{\partial^{3} \phi_{1}}{\partial \xi^{3}}+D \phi_{1}=F_{0} \cos (\omega \tau)
\end{gathered}
$$

where, $P=A l, Q=B l^{3}+C l\left(m^{2}+n^{2}\right)$,

The analytical solitary wave solution of the Eq. (134) as obtained in (68), is

$$
\phi_{1}=\phi_{m}(\tau) \operatorname{sech}^{2}\left(\frac{\xi-M(\tau) \tau}{W(\tau)}\right)
$$

where $\phi_{m}(\tau)=\frac{3 M(\tau)}{P}$ and $W(\tau)=2 \sqrt{\frac{Q}{M(\tau)}}$, with

$$
M(\tau)=\left(M-\frac{8 P F_{0}}{16 D^{2}+9 \omega^{2}}\right) e^{-\frac{4}{3} D \tau}+\frac{6 P F_{0}}{16 D^{2}+9 \omega^{2}}\left(\frac{4}{3} D \cos (\omega \tau)+\omega \sin (\omega \tau)\right)
$$

\section{Conclusions}

It is clear from the structure of the solitary wave solution of the DFKdV, DFMKdV and DFZK that the soliton amplitude and width depends on the nonlinearity and dispersion of the evolution equations, which are the function of different plasma parameter involve in the consider plasma system. Also evident from the structure of the approximate analytical solution that the amplitude and the width of the soliton depends on the Mach number $(M(\tau))$ which involve the forcing term $F_{0} \cos (\omega \tau)$ and the damping parameter. Thus the amplitude and the width of the solitary wave structure changes with the different plasma parameters. Also they are changes with the change of strength of external force $F_{0}$, frequency of the external force $\omega$ and the collisional frequency between the different plasma species. The effect of these parameter can be studied through numerical simulation. 


\section{Author details}

Laxmikanta Mandi ${ }^{1,2 \dagger}$, Kaushik Roy ${ }^{3 \dagger}$ and Prasanta Chatterjee ${ }^{1 *}$

1 Department of Mathematics, Visva-Bharati, Santiniketan, India

2 Department of Mathematics, Gushkara Mahavidyalaya, Guskhara, India

3 Beluti M.K.M. High School, Beluti, Birbhum, India

*Address all correspondence to: prasantacvb@gmail.com

$\uparrow$ These authors contributed equally.

\section{IntechOpen}

(C) 2020 The Author(s). Licensee IntechOpen. This chapter is distributed under the terms of the Creative Commons Attribution License (http://creativecommons.org/licenses/ by/3.0), which permits unrestricted use, distribution, and reproduction in any medium, provided the original work is properly cited. (c) BY 


\section{References}

[1] Russell JS. Report on waves. In: Murray J, editor. Report of the British Association for the Advancement of Science. 1944. pp. 311-390

[2] Korteweg DJ, de Vries G. On the change of form of long waves advancing in a rectangular canal, and on a new type of long stationary waves. Philosophical Magazine. 1985;39:422

[3] Zabusky NJ, Kruskal MD. Interaction of "solitons" in a collisionless plasma and the recurrence of initial states. Physical Review Letters. 1965;15: 240-243

[4] Sen A, Tiwary S, Mishra S, Kaw P. Nonlinear wave excitations by orbiting charged space debris objects. Advances in Space Research. 2015;56(3):429

[5] Chowdhury S, Mandi L, Chatterjee P. Effect of externally applied periodic force on ion acoustic waves in superthermal plasmas. Physics of Plasmas. 2018;25:042112

[6] Aslanov VS, Yudintsev VV. Dynamics, analytical solutions and choice of parameters for towed space debris with flexible appendages. Advances in Space Research. 2015; 55:660

[7] Israwi S, Kalisch H. Approximate conservation laws in the KdV equation. Physics Letters A. 2019;383:854

[8] Tsallis CJ. Possible generalization of Boltzmann-Gibbs statistics. Journal of Statistical Physics. 1988;52:479

[9] Zakharov VE, Kuznetsov EA. Three-dimensional solitons. Soviet Physics JETP. 1974;39:285 



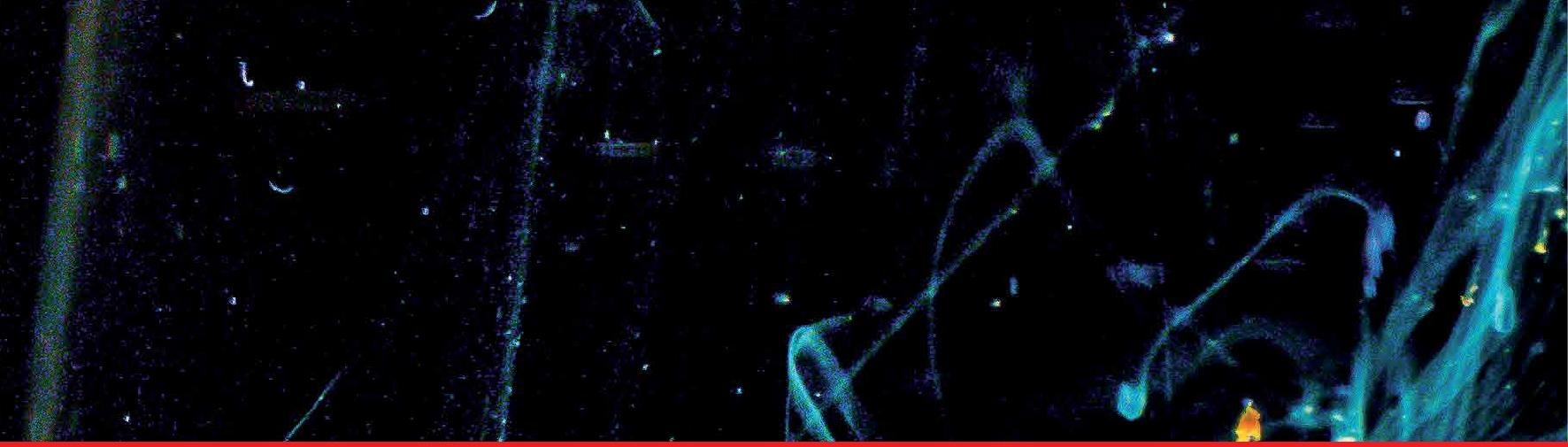

\section{Edited by Sukhmander Singh}

This book is planned to introduce the advances topics of plasma physics for research scholars and postgraduate students. This book deals with basic concepts in plasma physics, non-equilibrium plasma modeling, space plasma applications, and plasma diagnostics. It also provides an overview of the linear and nonlinear aspects of plasma physics. Chapters cover such topics as plasma application in space propulsion, microwave-plasma interaction, plasma antennas, solitary waves, and plasma diagnostic techniques.

\section{IntechOpen}

